VICTORIA UNIVERSITY OF WELLINGTON

Te Whare Wānanga o te Ūpoko o te Ika a Māui

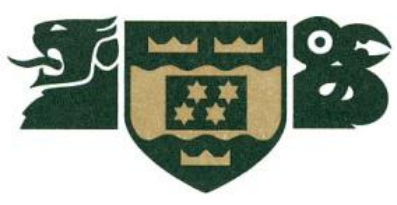

\title{
Coral bleaching resistance vs susceptibility: The role of antioxidant activity in symbiotic dinoflagellates
}

\section{Anne Wietheger}

A thesis submitted to Victoria University of Wellington in fulfilment of the requirements for the degree of Doctor of Philosophy in Science

Victoria University of Wellington 



\begin{abstract}
Coral bleaching, the loss of symbiotic dinoflagellate algae (genus Symbiodinium) and/or photosynthetic algal pigments from their coral host has become a regular occurrence in the last few decades due to increasing seawater temperatures. A key consideration in bleaching susceptibility is the symbiotic alga's physiology and its capacity to deal with abiotic stress; oxidative stress is of particular interest given that this can arise from thermally induced photosynthetic dysfunction.
\end{abstract}

The aim of this study was to compare the effects of thermal and oxidative stress on the photosynthetic performance of a range of Symbiodinium clades and types (i.e. sub-clades) in different states of symbiosis (in hospite, freshly isolated and in culture). Whether the responses to these two stressors are related was investigated; in particular, it was hypothesised that more thermally sensitive types would be more sensitive to oxidative stress. Furthermore, the study aimed to elucidate the role of antioxidants in the observed stress responses. The specific objectives were 1) to establish whether different types of cultured Symbiodinium have dissimilar sensitivities to oxidative stress, induced by hydrogen peroxide $\left(\mathrm{H}_{2} \mathrm{O}_{2}\right)$, and whether these are related to their thermal sensitivities; 2) measure the activity and relative amounts of specific reactive oxygen species (ROS) in different types of cultured Symbiodinium in response to thermal and oxidative stress induced by $\mathrm{H}_{2} \mathrm{O}_{2} ; 3$ ) measure total antioxidant activity in different cultured Symbiodinium types when under oxidative stress; and 4) compare and contrast the responses of different Symbiodinium types to thermal and oxidative stress when in hospite (i.e. in corals) and freshly isolated.

In this study, I showed that different Symbiodinium clades and types can differ widely in their responses to both thermal and oxidative stress. This was indicated by photosynthetic performance measured by chlorophyll fluorescence, and differences in the quantity of specific ROS measured via fluorescent probes and flow cytometry. For instance, when adding $\mathrm{H}_{2} \mathrm{O}_{2}$ to Symbiodinium F1, originally from Hawaii, a decrease of $>99 \%$ in maximum quantum yield $\left(F_{v} / F_{m}\right)$ was displayed, while there was no change in $F_{v} / F_{m}$ in the temperate Symbiodinium A1, freshly isolated from the anemone Anthopleura aureoradiata from New Zealand. When comparing the difference in ROS production between the control $\left(26^{\circ} \mathrm{C}\right)$ and a thermal stress treatment $\left(35^{\circ} \mathrm{C}\right)$, type E1 from Okinawa showed no 
difference in any of the measured ROS. In contrast, a different A1 type from the Gulf of Aqaba displayed an increase in the overall production of ROS, and more specifically in the production of superoxide. Symbiodinium types also displayed differential oxidative stress resistance, which was apparent from their antioxidant activities; in particular, total antioxidant capacity was measured by the ferric reducing antioxidant potential (FRAP) and cellular antioxidant activity (CAA) assays. For example, the aforementioned Symbiodinium types, A1 from the Gulf of Aqaba and F1, increased their antioxidant activities with increasing $\mathrm{H}_{2} \mathrm{O}_{2}$ concentrations. Meanwhile, type E1 displayed higher baseline levels of antioxidants in comparison to the other two types (A1, F1), which then decreased with increasing $\mathrm{H}_{2} \mathrm{O}_{2}$. Specific activities of superoxide dismutase and ascorbate peroxidase were also measured.

Stress susceptibility appears to be related both to Symbiodinium type and geographic origin, but greater sensitivity to thermal stress did not necessarily correlate with greater susceptibility to oxidative stress. The exact relationship between thermal and oxidative sensitivities in Symbiodinium spp. remains elusive, but it is suggested that different types might follow different strategies for dealing with stress. I propose that some Symbiodinium types rely more on photo-protection when exposed to thermal stress (and hence cope less with oxidative stress), while other types depend more on antioxidants and oxidative stress resistance. The latter might be the better strategy for types from more variable environments, such as higher latitude reefs or intertidal regions, where potentially stressful conditions may be encountered more frequently.

This study gives new insights into the variability of stress responses in the genus Symbiodinium, and the complex relationship between thermal and oxidative stress. The implications of these findings for coral bleaching susceptibility and the biogeographic distribution of different Symbiodinium types are discussed. 


\section{Contributions and Publications}

This thesis is written as a series of manuscripts, which are to be submitted in the near future. All laboratory work, field-work, analyses and writing were conducted by the Author, with assistance as described below.

Chapter 2: This chapter is formatted as a stand-alone manuscript and will be submitted for publication: Wietheger A., Fisher P.L., Gould K.S., Davy S.K. (in prep. for Journal of Experimental Botany). Variability in thermal and oxidative stress response in distinct Symbiodinium cultures

J. Howe assisted with some of the laboratory work. P.L. Fisher, K.S. Gould and S.K. Davy advised on experimental set-up, analyses and writing.

Chapter 3: This chapter is formatted as a stand-alone manuscript and will be submitted for publication: Wietheger A., Gould K.S., Davy S.K. (in prep.). ROS generation in response to thermal and oxidative stress in different Symbiodinium types.

K.S. Gould and S.K. Davy advised on experimental set-up, analyses and writing.

Chapter 4: This chapter is formatted as a stand-alone manuscript and will be submitted for publication: Wietheger A., Gould K.S., Davy S.K. (in prep.). Antioxidant potential in response to oxidative stress in different Symbiodinium types: comparison of FRAP and CAA assays

K.S. Gould and S.K. Davy advised on experimental set-up, analyses and writing.

Chapter 5: This chapter is formatted as a stand-alone manuscript and will be submitted for publication: Wietheger A., Fisher P.L., Gould K.S., Davy S.K. (in prep.). Thermal and oxidative stress responses of different Symbiodinium types when in different states of symbiosis

P.L. Fisher, K.S. Gould and S.K. Davy advised on experimental set-up, analyses and writing. 


\section{Acknowledgments}

Foremost, I would like to thank my supervisor Dr Simon Davy who has made this thesis possible. Despite his busy schedule, Simon's door was always open and he was there to listen and give feedback. I would also like to thank my second supervisor Prof Kevin Gould who always lent me support and a sympathetic ear in my times of crisis.

For the supply of the Symbiodinium cultures for my lab experiments, I would like to thank Scott Santos (Auburn University, Auburn, AL, USA), Mary Alice Coffroth (University of Buffalo, Buffalo, NY, USA) and Gisele Muller-Parker (former Western Washington University, WA, USA).

While in Australia, Prof Peter Ralph and Dr Ross Hill welcomed me into their lab at the University of Technology, Sydney, which was greatly appreciated; as was the assistance provided by all who helped me during my fieldwork experience on Heron Island and kept me from leaving the island after 3 days: Dr Selina Ward, Ben McIntosh, Chris Doropoulos, Mirta Zupan, Alicia Lloyd and Catalina Reyes.

The realization of core and supplementary research for this thesis was possible due to funding by a Victoria PhD Scholarship, Faculty Strategic Research Grants, a Kathleen Stewart Scholarship, a Wellington Botanical Society student grant and a Victoria University Submission Scholarship.

Next, I wish to thank my lab group for their support and help - as well as for all those coffee breaks which gave me something to look forward to each morning. For help during the critical last phase, for helping with data and editing, I want to thank Susanne Becker and Thomas Krüger. I owe a special thanks to our post-doc Dr Paul Fisher who picked up a lot of the pieces over the years, helping me all along the way.

I will be eternally grateful for the endless support, provision of coffee, distractions and wine from my friends in Wellington, which kept me on the side of sanity in these long and often tough years; these are Jennifer Howe, Dorota Starzak, Stacey Martin, Scott Lawrence, Emma Gibbin, Penny Tok, Danelle Lekan, Rafael Barbieri, Lynn Jordan, Kart James, and especially Stefanie Pontasch for always being there. 
I also need to thank my 'old' friends all over the world who lent support and encouragement whenever necessary and kept believing in me when I did not: Annette Reus, Alice Fertig, Natascha Geistmann, Verena Seuffert, Ralf Aurahs, Katrin Sens, Claire Achmad and Jolanda v/d Woud.

Lastly, a very special thanks goes out to my parents, Michael and Ursula, and my evergrowing family, Lena, Sven and Ida, Jörg and Lucia, Peter and Katrin. I could not have done it without your moral and financial support over all these years. Despite the many miles between us, I'm thankful to still be a part of this family. 


\section{List of Figures}

Figure 1.1 - Light micrograph image of Symbiodinium sp.

Figure 1.2 - Schematic phylogenetic tree of the genus Symbiodinium

Figure 1.3 - Conceptual graph showing different mechanisms of symbiont loss from host tissue

Figure 1.4 - Conceptual graph of three sites of initial damage in the photosystems of symbiotic dinoflagellates due to temperature and light

Figure 1.5 - Generation of different reactive oxygen species (ROS)

Figure 1.6 - Differential bleaching by colonies of the same coral species

Figure 2.1 - Graph showing the effect of increasing temperature on photosynthetic capacity of Symbiodinium types

Figure 2.2 - The effect of increasing hydrogen peroxide $\left(\mathrm{H}_{2} \mathrm{O}_{2}\right)$ on photosynthetic capacity of Symbiodinium types

Figure 2.3 - The effect of increasing hydrogen peroxide $\left(\mathrm{H}_{2} \mathrm{O}_{2}\right)$ on oxygen fluxes of Symbiodinium types

Figure 3.1 - Confocal microscope images of Symbiodinium cells with different fluorescent probes

Figure 3.2 - Reactive oxygen species (ROS) production over time by different Symbiodinium types when exposed to elevated temperature

Figure 3.3 - Flow cytometer picture of Symbiodinium cells stained with fluorescent probe after exposure to $\mathrm{H}_{2} \mathrm{O}_{2}$

Figure 3.4 - Reactive oxygen species (ROS) production over time by different Symbiodinium types when exposed to hydrogen peroxide

Figure 4.1 - Ferric reducing antioxidant potential (FRAP) values per cell volume for three Symbiodinium types

Figure 4.2 - Fluorescence units for a Symbiodinium type after addition of hydrogen peroxide $\left(\mathrm{H}_{2} \mathrm{O}_{2}\right)$ and the fluorescent probe $\mathrm{H}_{2}$ DCF-DA

Figure 4.3 - Cellular antioxidant assay (CAA) fluorescent units per cell volume for Symbiodinium types

Figure 5.1 - Maximum quantum yield $\left(\mathrm{F}_{\mathrm{v}} / \mathrm{F}_{\mathrm{m}}\right)$ in Symbiodinium types in hospite at different temperatures and $\mathrm{H}_{2} \mathrm{O}_{2}$ exposure 
Figure 5.2 - Symbiodinium cell densities and combined chlorophyll $a+\mathrm{c}_{2}$ concentration for five Symbiodinium types

Figure 5.3 - Maximum quantum yield $\left(\mathrm{F}_{\mathrm{v}} / \mathrm{F}_{\mathrm{m}}\right)$ and total antioxidant potential (FRAP) in two types of freshly isolated Symbiodinium spp.

Figure 5.4 - Superoxide dismutase (SOD) and ascorbate peroxidase (APX) activity at different temperatures for Symbiodinium in hospite

Figure 5.5 - Antioxidant activity at different hydrogen peroxide $\left(\mathrm{H}_{2} \mathrm{O}_{2}\right)$ concentrations in five different Symbiodinium types in hospite

Figure 6.1 - Conceptual diagram showing relationship between thermal and oxidative stress in tolerant and susceptible types of Symbiodinium sp.

Figure B.1 - The effect of increasing temperature on photosynthetic capacity of Symbiodinium types over time

Figure B.2 - The effect of increasing hydrogen peroxide $\left(\mathrm{H}_{2} \mathrm{O}_{2}\right)$ on photosynthetic capacity of Symbiodinium types over time

Figure B.3 - The effect of increasing hydrogen peroxide $\left(\mathrm{H}_{2} \mathrm{O}_{2}\right)$ on photosynthetic capacity of Symbiodinium culture strains over time

Figure C.1 - Ferric reducing antioxidant potential (FRAP) values three Symbiodinium types

Figure C.2 - Cellular antioxidant assay (CAA) fluorescent units for two Symbiodinium types 


\section{List of Tables}

Table 2.1 - Symbiodinium cultures and freshly isolated Symbiodinium with corresponding host species, geographic origin and sub-clade.

Table 2.2 - Summary table of results from Chapter 2

Table 3.1 - Symbiodinium cultures with corresponding host species, geographic origin and sub-clade.

Table 4.1 -Symbiodinium cultures with corresponding host, geographic origin and subclade

Table 5.1 - Symbiodinium sub-clades present in coral species from Heron Island

Table 6.1 - Summary table of results from Chapters 2- 4

Table A1 - Complete list of used Symbiodinium cultures with corresponding sub-clade, host, geographic origin, GenBank number and sequence length 165

Table A.2 - Symbiodinium sub-clades present in coral species from Heron Island 169 


\section{Abbreviations}

$\mathrm{O}_{2}$

${ }^{1} \mathrm{O}_{2}$

ANOVA

APX

ATP

CAA

CAT

Chl

$\mathrm{CO}_{2}$

$\mathrm{Cu} / \mathrm{ZnSOD}$

DCF

DCMU

DHA

DMS

DMSO

DMSP

DNA

EDTA

$\mathrm{ETR}_{\max }$

FeSOD

Fp

FRAP

FSW

$\mathrm{F}_{\mathrm{v}} / \mathrm{F}_{\mathrm{m}}$

GSH
Oxygen

Singlet oxygen

Analysis of Variance

Ascorbate peroxidase

Adenosine triphosphate

Cellular antioxidant assay

Catalase

Chlorophyll

Carbon dioxide

Copper and zinc superoxide dismutase

2',7'-dichlorofluorescein

3-(3,4-dichlorophenyl)-1,1-dimethylurea

Dehydroascorbate

Dimethyl sulfide

Dimethyl sulfoxide

Dimethylsulfoniopropionate

Desoxyribonucleic acid

Ethylenediaminetetraacetic acid

Maximum electron transport rate

Iron superoxide dismutase

Fluorescent pigment

Ferric reducing antioxidant potential assay

Filtered seawater

Maximum quantum yield of PSII

Glutathione reduced 
GSSG

$\mathrm{H}_{2}$ DCF-DA

$\mathrm{H}_{2} \mathrm{O}_{2}$

$\mathrm{HCl}$

HI

Hsp

IPAM

ITS

$\mathrm{kDA}$

MAA

MDA

$\mu \mathrm{L}$

$\mathrm{mL}$

$\mu \mathrm{M}$

$\mathrm{mM}$

MnSOD

N/A

$\mathrm{NADPH}$

NBT

NCMA

NOAA

NPQ

$\mathrm{O}_{2}^{-}$

$\mathrm{OH}^{\bullet}$

$\mathrm{P}$

PAR

PCR
Glutathione oxidised

2',7'-dichlorodihydrofluorescein diacetate

Hydrogen peroxide

Hydrochloric acid

Heron Island

Heat shock protein

Imaging pulse amplitude modulated chlorophyll fluorometer

Internal transcribed spacer

Kilo Dalton

Mycosporine-like amino acid

Monodehydroascorbate

Microlitre

Millilitre

Micromolar

Millimolar

Manganese superoxide dismutase

Not available

Nicotinamide adenine dinucleotide phosphate (reduced)

NitroBlue Tetrazolium chloride

National Center for Marine Algae and Microbiota

National Oceanic and Atmospheric Administration

Non-photochemical quenching

Superoxide

Hydroxyl radical

Photosynthetic production

Photosynthetically active radiation $(400-700 \mathrm{~nm})$

Polymerase chain reaction 
Abbreviations

PSI +II

$\mathrm{Q}_{\mathrm{m}}$

$\mathrm{R}$

rDNA

RLC

RNA

ROS

Rubisco

SE

SOD

SOG

TPTZ

UVR
Photosystem I and II

Maximum excitation pressure over PSII

Respiratory consumption

Ribosomal DNA

Rapid light curve

Ribonucleic acid

Reactive oxygen species

Ribulose-1,5-bisphosphate carboxylase oxygenase

Standard error

Superoxide dismutase

Singlet oxygen sensor green

Tripyridyltriazine

Ultraviolet radiation (280- $400 \mathrm{~nm})$ 


\section{Table of Contents}

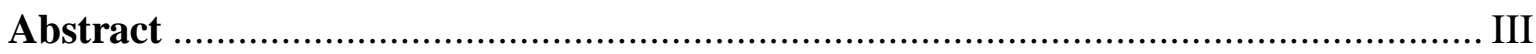

Contributions and Publications ....................................................................... V

Acknowledgments....................................................................................... VII

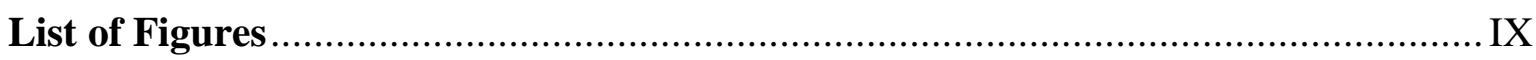

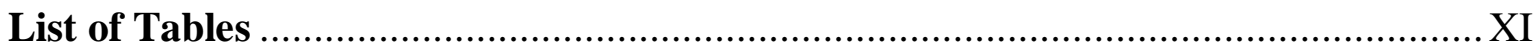

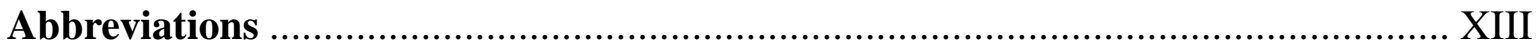

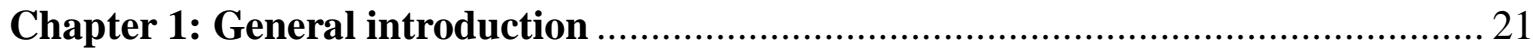

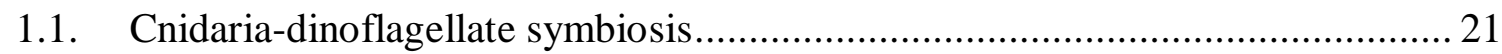



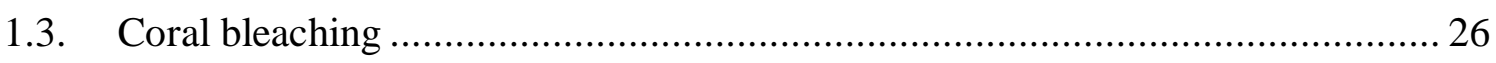

1.4. Oxidative stress and Reactive Oxygen Species (ROS) ……………………...... 31

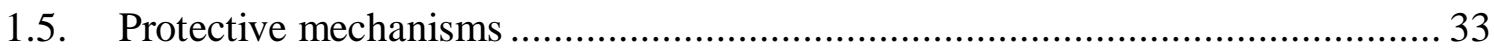

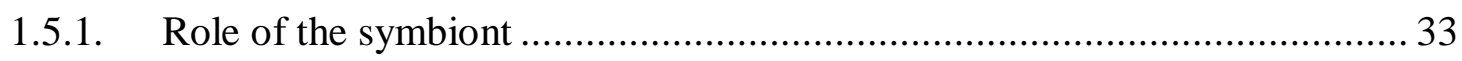

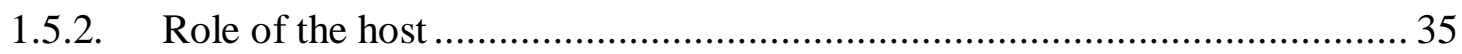

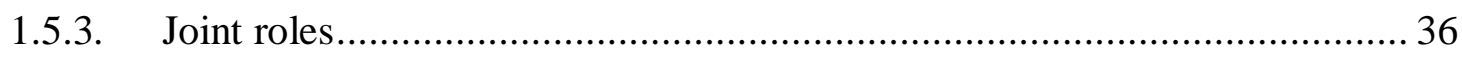

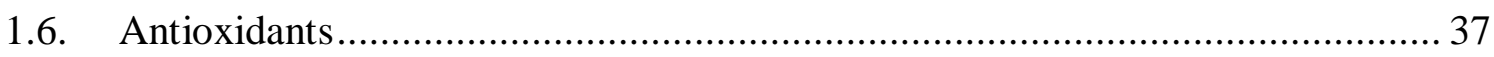

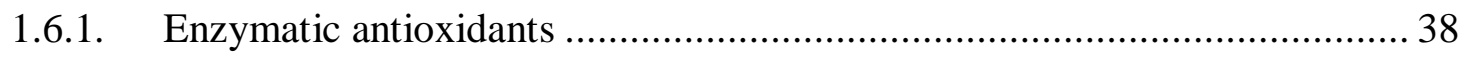

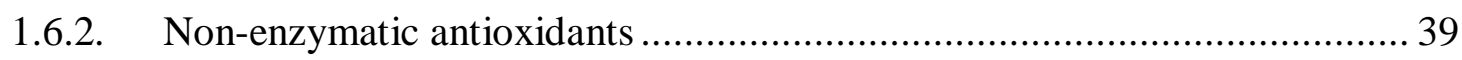

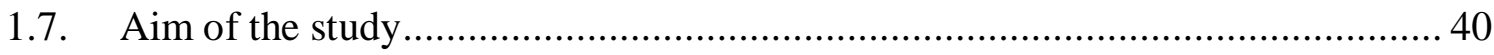

Chapter 2: Variability in thermal and oxidative stress response in distinct

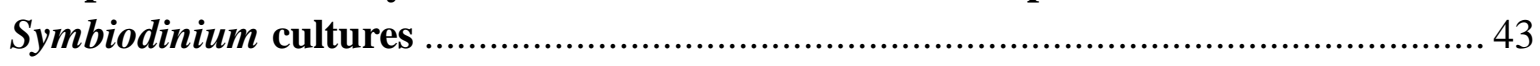

2.1. Introduction............................................................................................. 43

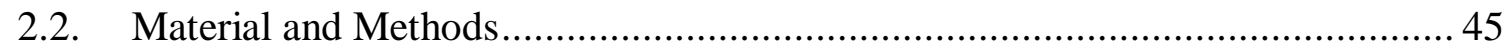

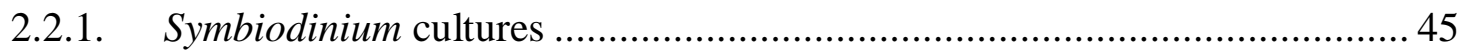

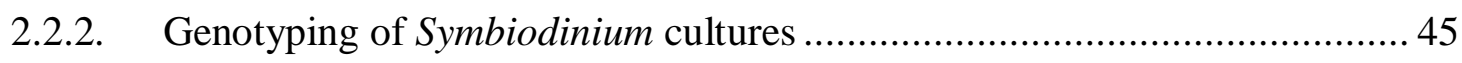

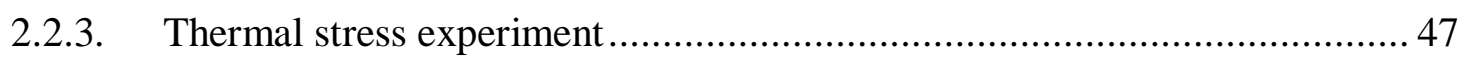

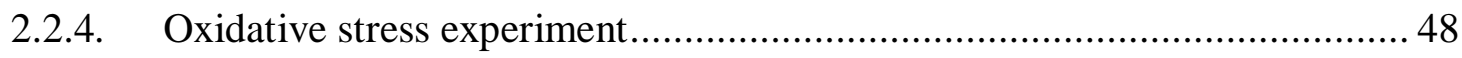

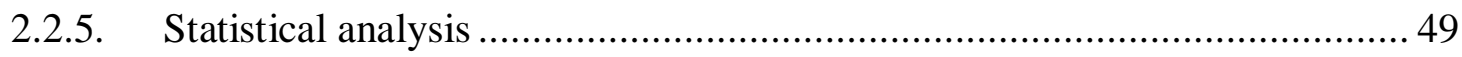

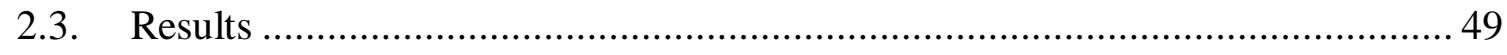

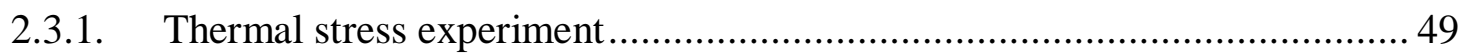

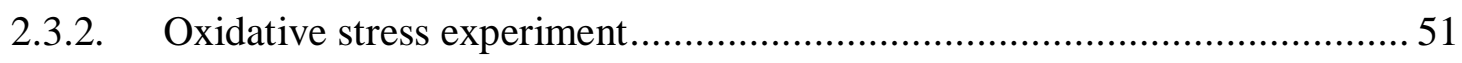

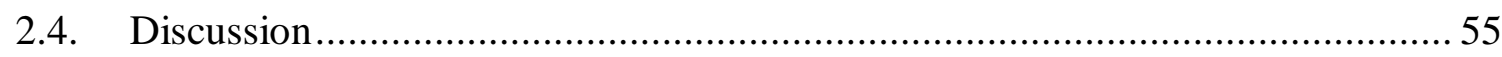


Chapter 3: ROS generation in response to thermal and oxidative stress in different



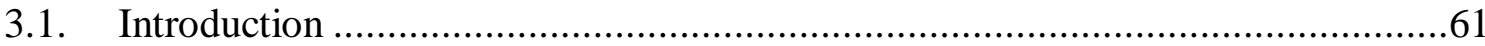

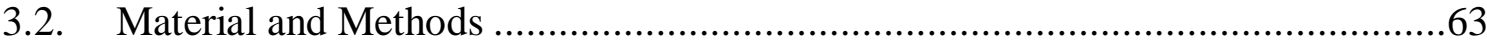

3.2.1. Symbiodinium types and cultivation ..................................................63

3.2.2. Genetic identification of Symbiodinium types.........................................63

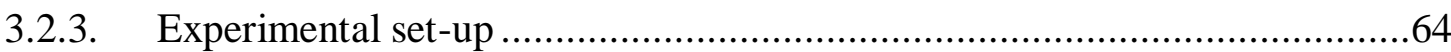

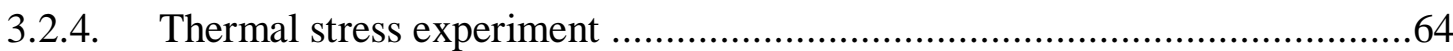

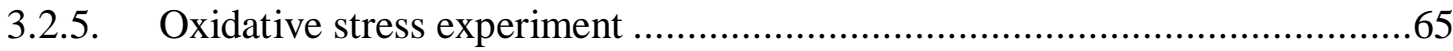

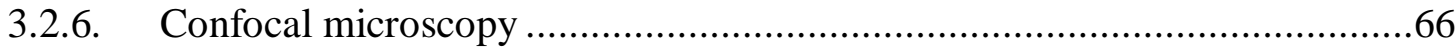

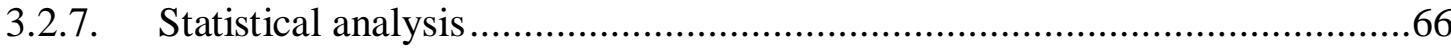

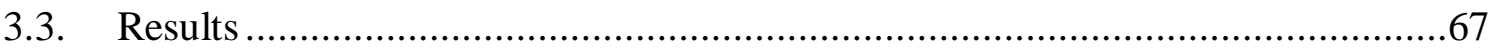

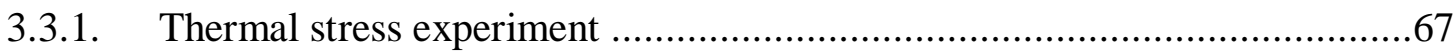

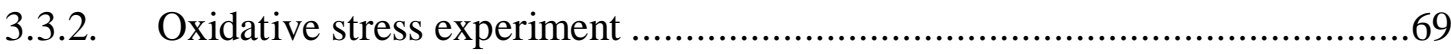

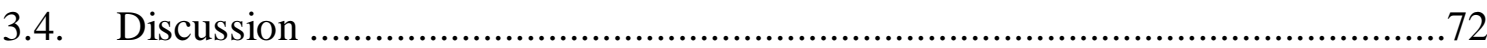

Chapter 4: Antioxidant potential in response to oxidative stress in different Symbiodinium types: comparison of FRAP and CAA assays ...................................79

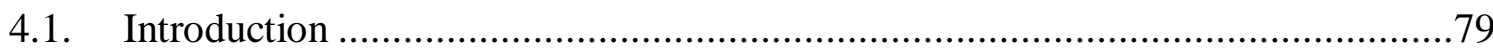

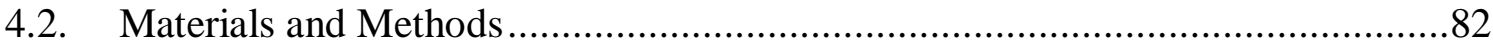

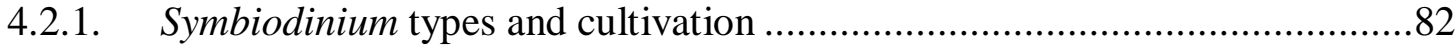

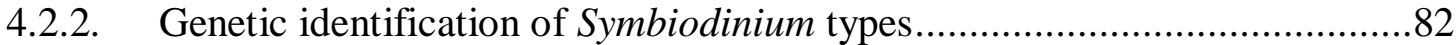

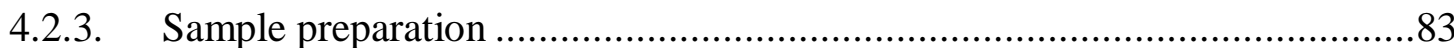

4.2.4. Ferric reducing antioxidant potential (FRAP) assay.................................83

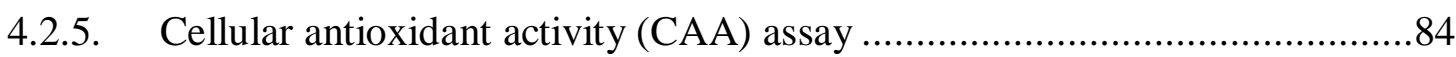

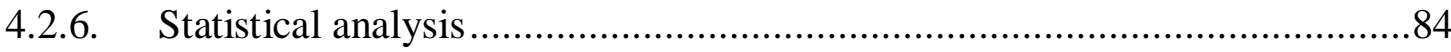

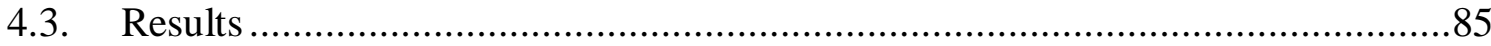

4.3.1. Ferric reducing antioxidant potential (FRAP) assay.................................85

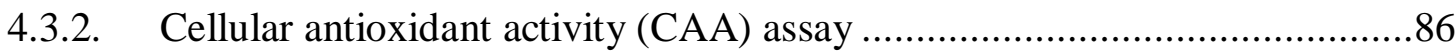

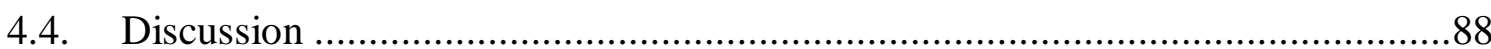

Chapter 5: Thermal and oxidative stress responses of different Symbiodinium types

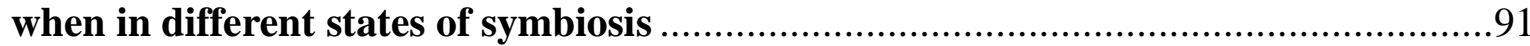

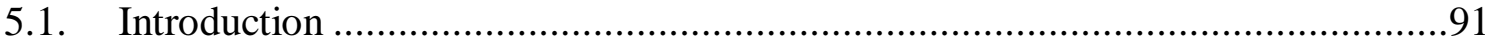

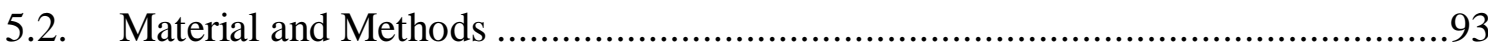

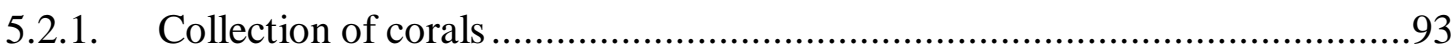


5.2.2. Thermal stress experiment with Symbiodinium sp. in hospite..................... 94

5.2.3. Oxidative stress experiment with Symbiodinium sp. in hospite ................... 95

5.2.4. Thermal and oxidative stress experiments with freshly isolated

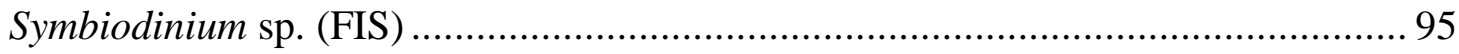

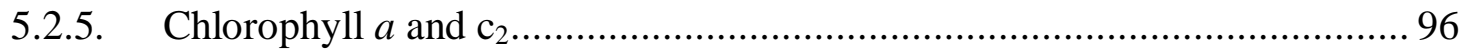

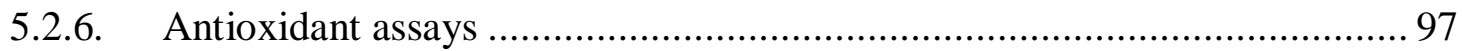

5.2.7. Genetic identification of Symbiodinium types........................................ 99

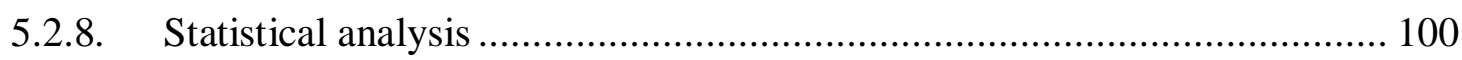



5.3.1. Photosynthetic parameters for Symbiodinium sp. in hospite ...................... 100

5.3.2. Photosynthetic parameters for freshly isolated Symbiodinium .................. 104

5.3.3. Antioxidant capacity in Symbiodinium cells in hospite ............................ 106

5.3.4. Antioxidant capacity (FRAP) in freshly isolated Symbiodinium................ 108

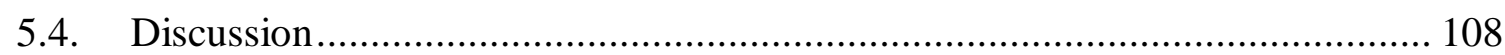

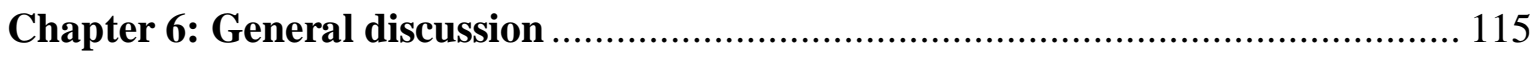

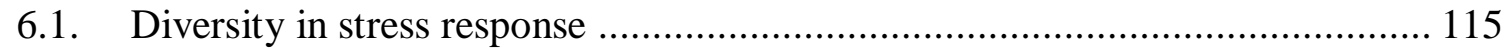

6.2. Protection from ROS - the special role of antioxidants …........................... 120

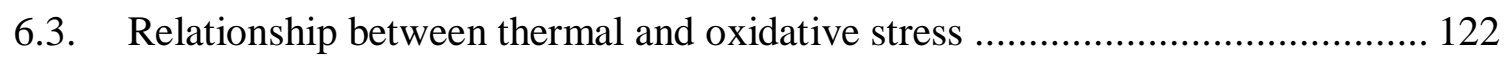

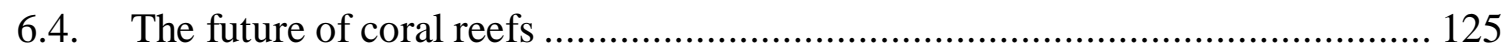

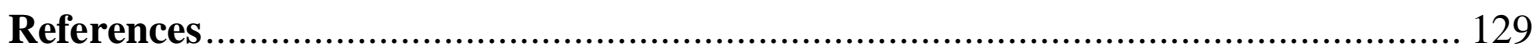

Appendix

Appendix A - Molecular Typing of Symbiodinium .............................................. 165

Appendix B - Additional chlorphyll fluorescence data (Chapter 2)........................... 171

Appendix C - Additional FRAP and CAA data (Chapter 4)..................................... 184 


\section{Chapter 1: General introduction}

\subsection{Cnidaria-dinoflagellate symbiosis}

'A coral reef cannot be properly described. It must be seen to be thoroughly appreciated.' (Sidney Hickson 1889).

For millennia, humans have been captured by the beauty of tropical and subtropical coral reefs. These natural structures occupy only $0.1 \%$ of the ocean's surface area and are largely restricted to the uppermost 25 meters, yet they are home to $25 \%$ of all marine species. This high biodiversity (Hoegh-Guldberg et al. 2007) is even more intriguing as reefs are situated in the nutrient-poor 'blue deserts' of the oceans. Additionally, coral reefs play important economic roles through fisheries, coastal protection and tourism (HoeghGuldberg et al. 2007), accounting globally for up to US\$375 billion a year (NOAA 2010). On the other hand, the destruction of just one $\mathrm{km}$ of coral reef could cost between US\$137,000 and US\$1.2 million over a 25-year period, according to the environmental organization WWF (www.worldwildlife.org).

Key to the success of the scleractinian (stony) corals as modern reef-builders is the complex association between these animals and their dinoflagellate symbionts of the genus Symbiodinium (Fig. 1.1). This close physical interaction between organisms of different species, or 'symbiosis', is a case of mutualism because both partners, the coral host as well as the algal symbiont, benefit from the relationship (Boucher 1985). The presence of these microalgae contributes significantly to the productivity, survival and success of their hosts (HoeghGuldberg et al. 2007, Davy et al. 2012). The algae, often called 'zooxanthellae', live as endosymbionts inside the gastrodermal host cells (Gates et al. 1992) surrounded by a host-derived membrane; this

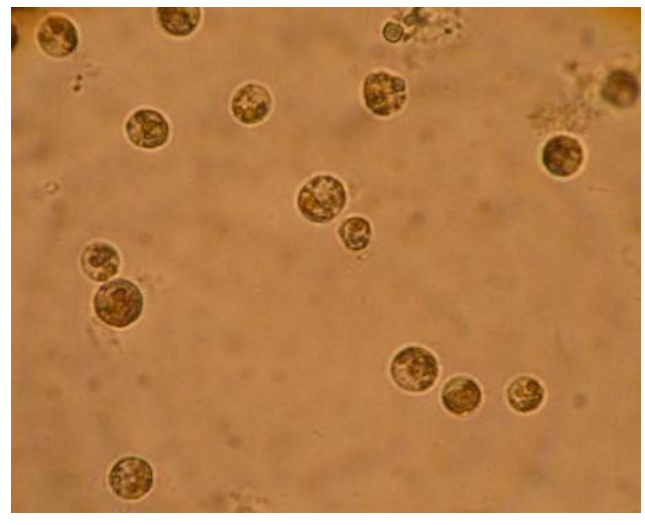

Figure 1.1: Light micrograph of Symbiodinium sp. The brown dinoflagellate cells are approximately $10 \mu \mathrm{m}$ in diameter. 
entire vacuolar structure is known as the symbiosome (Yellowlees et al. 2008). These autotrophic organisms provide photosynthetically-fixed carbon and oxygen to their hosts (Gates et al. 1995, Wang and Douglas 1997, Venn et al. 2008). In return, inorganic nutrients $\left(\mathrm{CO}_{2}, \mathrm{NH}_{3}, \mathrm{PO}_{4}\right)$ released by the host as waste are used by the algae to synthesize complex organic compounds, such as glycerol, sugars, organic acids and amino acids. Again, the generated products are returned to the host to supplement its metabolism (Gates et al. 1995, Yellowlees et al. 2008). This successful symbiosis is aided by the animal's body plan, with a large surface area to volume ratio that promotes light capture (Venn et al. 2008). In total, more than $100 \%$ of the metabolic requirements of the coral host can be provided by its symbionts under well-illuminated conditions, thus ensuring its survival and growth (Muscatine et al. 1984, Hoegh-Guldberg et al. 2007).

Depending on the coral species, Symbiodinium cells are acquired in two different ways. Firstly, genetically-identical algae can be inherited from the mother colony, by so-called vertical transmission (Szmant-Froelich et al. 1985). Secondly, horizontal transmission exists, which sees the uptake of algae from the surrounding environment (Coffroth et al. 2001, Rodriguez-Lanetty et al. 2006). The latter mode is far more common and is employed by about $85 \%$ of cnidarians (Weis et al. 2000).

The onset of symbiosis includes a series of complex steps in a process referred to as 'the winnowing' that range from molecular signalling involved in inter-partner recognition to inter-microbe ecological interactions (Wood-Charlson et al. 2006, Fransolet et al. 2012). One well-known microbe-associated molecular pattern (MAMP)-pattern recognition receptor (PRR) interaction in the cnidarian-dinoflagellate symbiosis is between cell-surface glycans of Symbiodinium and lectins in the host genome (Logan et al. 2010, Davy et al. 2012). Glycan-lectin signalling may determine whether a symbiosis is established and Symbiodinium cells are engulfed into the host's endodermal cells by phagocytosis (Schwarz et al. 1999). MAMP-PRR interaction might also play a role in the stabilization of the symbiotic relationship and the arrest of the algae in a non-motile stage while cell division is maintained (Koike et al. 2004). Digestion of Symbiodinium cells in the symbiosome by the host must be prevented, probably by manipulation of endosomal trafficking by the dinoflagellate (Fitt and Trench 1983, Chen et al. 2004, Venn et al. 2009). Inside the host, the algae are coccoid in shape and without flagella, unlike free-living 
dinoflagellates (Perez 2007). In the majority, host cells contain a single symbiont (Muscatine et al. 1998), while coral colonies average $1.54 \times 10^{6}$ dinoflagellates $\mathrm{cm}^{-2}$ coral surface (Titlyanov et al. 1996). Symbiont cell numbers are likely maintained by asexual division, as sexual reproduction has not been observed so far and a dynamic equilibrium between symbiont proliferation and host cell growth is maintained (Yellowlees et al. 2008). In order to control symbiont numbers, excess or unhealthy symbionts are expelled or actively degraded from the host's growing edges (Fitt 2000) and algal cell division can be inhibited also (Fishman et al. 2008). Additionally, symbiont density varies seasonally and is usually inversely correlated with increases in temperature and light (Fitt et al. 2000).

\subsection{Symbiodinium diversity}

When first formally classified, the symbiotic dinoflagellates assigned to the genus Symbiodinium Freudenthal were all thought to be one pandemic species, Symbiodinium microadriaticum (Freudenthal 1962). This classification was subsequently questioned as a result of behavioural, physiological and ultrastructural studies in the 1980s (Schoenberg and Trench 1980a, b, Trench and Blank 1987, Wilkerson et al. 1988) as well as in molecular studies in the 1990s and beyond (Rowan and Powers 1992, Rowan and Knowlton 1995, Santos et al. 2001, Baker 2003, Santos et al. 2004). Most recently, by comparing nuclear and chloroplast ribosomal DNA (rDNA), nine genetically distinct lineages (called clades A-I) containing a high number of sub-clades ('types') based on internal transcribed spacer (ITS) regions, have been identified (Fig. 1.2; LaJeunesse 2001, Coffroth and Santos 2005, Pochon and Gates 2010). With the introduction of new Symbiodinium clades and an increasing number of types over the years, some confusion has arisen over the ideal groupings and the relation of the taxa within the individual clades (Baker 2003). Also, many minimally divergent variants probably represent intraspecific variations that have diverged ancestrally rather than real types (LaJeunesse 2005, Thornhill et al. 2007, Sampayo et al. 2008, Correa and Baker 2008). A systematic revision of the Symbiodinium clades using modern molecular techniques and a combination of sequences (mitochondrial, chloroplast, ribosomal genes, ITS and plastid) might possibly resolve some of these problems and dramatically reduce type numbers (Weis et al. 2008, LaJeunesse and Thornhill 2011). 


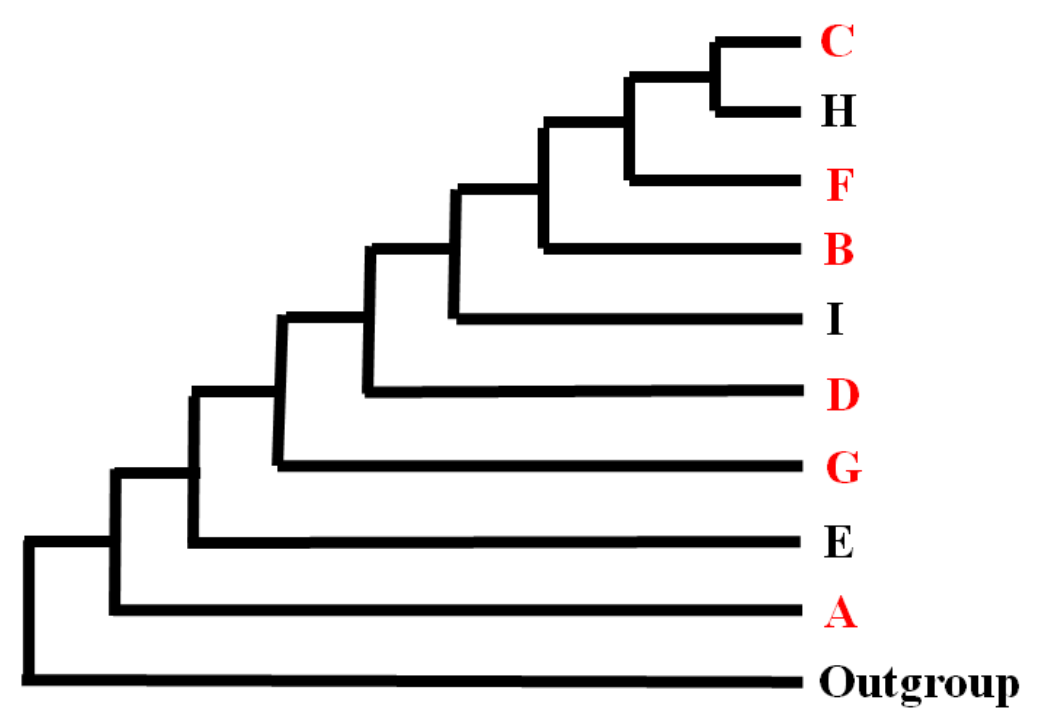

Figure 1.2: Schematic phylogenetic tree of the genus Symbiodinium, showing the known clades A-I, based on nuclear and chloroplastic markers. Highlighted in red are those clades/lineages that associate with scleractinian corals. Modified after Coffroth and Santos (2005) and Pochon et al. (2010).

The Symbiodinium types found in different taxa of cnidarians are not uniform, with scleractinian corals containing clades $\mathrm{A}, \mathrm{B}, \mathrm{C}, \mathrm{D}, \mathrm{F}$ and $\mathrm{G}$, sea anemones containing A, B, C, D and E, and zoanthids containing A, B, C and D (Baker 2003). Moreover, even though one scleractinian coral colony can host multiple symbiont types (Rowan and Knowlton 1995, Goulet and Coffroth 1997, Rowan et al. 1997, Coffroth et al. 2001, Santos et al. 2001), the ratio of observed to possible host-symbiont combinations is very small (Baker 2003). Coral hosts may also associate with different Symbiodinium types depending on the environmental conditions, so influencing the fitness of the holobiont (i.e. the coral and its community of symbiotic partners; Mieog et al. 2009). Indeed, it is clear that even closelyrelated Symbiodinium types can differ widely in their physiological responses to environmental factors (especially temperature and light) or host specificity (Sampayo et al. 2008). This will often be reflected in the alga's genetic code, with small differentiations, such as the aforementioned intraspecific variations (Smith-Keune and Van Oppen 2006, Thornhill et al. 2007). As a consequence of such niche diversification, vertical as well as horizontal distribution patterns of Symbiodinium types form (Rowan and Knowlton 1995, Rowan et al. 1997, Iglesias-Prieto et al. 2004, Sampayo et al. 2007, LaJeunesse et al. 
2010). Still, not all coral species can associate with different Symbiodinium types, presumably as a result of the host-symbiont recognition mechanisms. While there is the potential for environmental acclimation, i.e. physiological adjustment, of symbiont cells (Ulstrup et al. 2006), this 'monotyping' will likely make the coral holobiont more susceptible and less adaptable to environmental changes, especially drastic ones caused by climate change conditions (Weis 2010).

The initial uptake of dinoflagellates by juvenile corals during natural infection is not necessarily genetically limited; lineages, or 'clades', of Symbiodinium that are distinct from the parental assemblage can be acquired (Coffroth et al. 2001, Belda-Baillie et al. 2002). Dinoflagellate diversity does not seem to depend on the mode of symbiont acquisition, as acroporid coral species with horizontal symbiont transmission do not harbour a more diverse symbiont population than those with vertical transmission (Van Oppen 2004). However, the association between coral host and algal symbiont usually remains flexible. A change in Symbiodinium assemblage can occur over time, apparently in response to the prevailing environmental conditions (Rowan and Knowlton 1995, Coffroth et al. 2001, Baker 2003).

In early studies, generalisations about single Symbiodinium clades were made, such as referring to them as 'invasive/opportunistic' for Clade A, 'sun specialists' for B, 'shade specialists' for C and 'stress-tolerant' for E (Rowan 1998, Toller et al. 2001). This, however, proved to be premature when new information about intra-cladal variation emerged (Savage et al. 2002). Still, broad-scale inter-cladal patterns are apparent, with some taxa being more widely distributed among different hosts as well as across geographic regions than others; these can be considered as 'generalists' and 'specialists', respectively (LaJeunesse 2001, Stat et al. 2006, McCabe Reynolds et al. 2009). For instance, in some coral species, members of Symbiodinium clades A, B and/or F appear to be more abundant at higher latitudes while Symbiodinium clade $\mathrm{C}$ is more prevalent at tropical latitudes (Rodriguez-Lanetty et al. 2001, Savage et al. 2002). However, in other studies, no Symbiodinium clade diversity has been found over wide latitudinal ranges and differences occur only on sub-cladal level (LaJeunesse et al. 2004, Chen et al. 2005, LaJeunesse et al. 2008) 


\subsection{Coral bleaching}

Coral reefs are one of the most vulnerable marine ecosystems, despite their importance and persistence over geological time (Hoegh-Guldberg et al. 1999). In recent years, a massive decline of coral reefs has been occurring, with an estimated $30 \%$ already severely damaged and a possible loss of almost $60 \%$ by 2030 (Hughes et al. 2003, Donner 2009, Pandolfi et al. 2011).

Reefs are frequently threatened by natural events like storms and sedimentation, but they are able to cope with these sporadic events. A much more important factor is the increasing anthropogenic disturbance of the last few decades. Local influences include sewage effluent, eutrophication (Grigg 1994) and overfishing (Norström et al. 2009). On a global scale, coral reefs are facing global warming (Hughes et al. 2003, Hoegh-Guldberg et al. 2007, Harrison et al. 2011) and ocean acidification (Hoegh-Guldberg et al. 2007, Kleypas and Yates 2009, Crawley et al. 2010), both of which are caused by increasing levels of greenhouse gases (especially $\mathrm{CO}_{2}$ ) in the atmosphere. Of most relevance to this thesis, increased seawater temperatures related to global warming may cause mass coral bleaching events and the death of large expanses of coral reef (Hoegh-Guldberg 1999, HoeghGuldberg et al. 2007).

Coral bleaching is the loss of the symbiotic dinoflagellates and/or their photosynthetic pigments in response to stress; hence the coral loses its colour and the original calcium carbonate skeleton becomes apparent (Gates et al. 1992, Douglas 2003). Bleaching can be induced by a range of environmental stresses such as high UV radiation, extreme salinity and microbial infection (Berkelmans and Oliver 1999, Kerswell and Jones 2003, Rosenberg and Falkovitz 2004) but the major stress is higher-than-normal seawater temperature (Hoegh-Guldberg and Smith 1989, Gates et al. 1992, Perez et al. 2001), in combination with high light intensity (Hill and Ralph 2007, Ainsworth et al. 2008). Indeed, as corals and other tropical symbioses with Symbiodinium spp. live close to their upper thermal limits, an increase in just a few degrees Celsius can cause bleaching (Fitt et al. 2001). Consequences of bleaching are decreased growth, increased susceptibility to disease and dramatically increased mortality of the symbiotic partners (Hoegh-Guldberg et al. 2007, Weis 2008). 
A range of cellular mechanisms by which symbionts are lost from host tissue have been proposed (Fig. 1.3): 1) in situ degradation; this could include symbionts degrading or dying as a consequence of ROS, or the host actively destroying or expelling the symbiont as part of a host innate immune response (Dunn et al. 2004); 2) exocytosis of symbionts about which very little is known (Fang et al. 1997); 3) host cell detachment of whole living cells with the symbiont still inside, possibly as a consequence of host cell death (Gates et al. 1992); 4) apoptosis, i.e. controlled cell death, an orderly set of events including morphological alterations such as cell shrinkage, DNA fragmentation and the formation of apoptotic bodies containing cell debris; possible reasons for cell death are removal of damaged tissue and the aforementioned innate immune response (Richier et al. 2006, Dunn and Weis 2009, Tchernov et al. 2011); 5) necrosis, i.e. uncontrolled cell death caused by rupture of the plasma as a consequence of swelling of the cell due to extrinsic factors; cellular material is released (Douglas 2003, Strychar et al. 2004). However, it is still unknown how these mechanisms fit into the larger environmental picture of bleaching (Weis 2008). For example, there is no agreement upon which mechanism dominates in

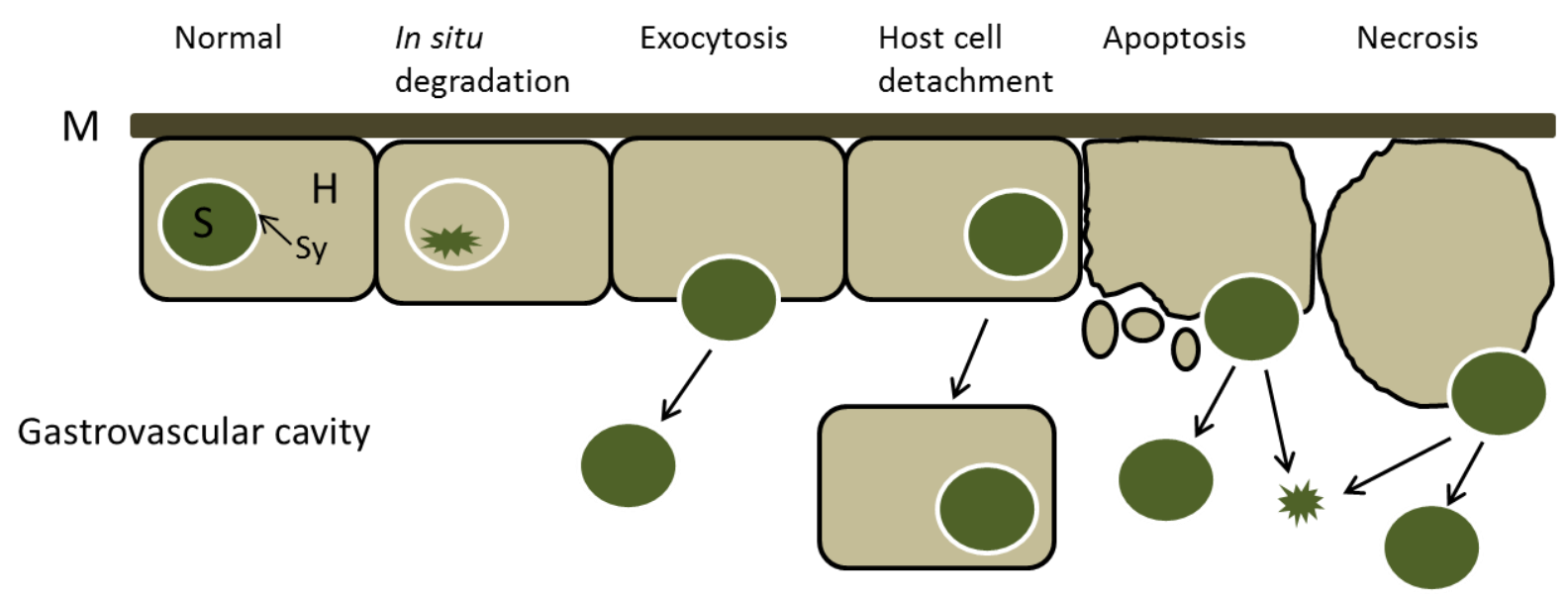

Figure 1.3: Different mechanisms of symbiont loss from host tissue as discussed in the text. From left to right, symbionts are lost by in situ degradation including symbiont expulsion (not shown), exocytosis of symbiont cells, host cell detachment, apoptosis or necrosis. Normal host cells $(\mathrm{H})$ include healthy dinoflagellate symbiont (S) surrounded by the symbiosome (Sy). Host cells are attached to the cell-free mesoglea $(\mathrm{M})$, while any cells or cell debris is released into the gastrovascular cavity. Figure adapted from Weis (2008). 
nature, if the different mechanisms are connected to differing types of stress, how the occurrence of these differing types varies between host and/or symbiont taxa, and how and if different mechanisms interact (Weis 2008).

While the underlying cellular mechanisms responsible for symbiont loss have not yet been fully identified (Downs et al. 2002, Weis et al. 2008), it has been suggested that the initial dysfunction occurs when high temperature causes a negative impact on the light (Warner et al. 1999, Tchernov et al. 2004) or alternatively the dark reactions of photosynthesis (Jones et al. 1998, Venn et al. 2008). Photosynthesis in general is separated into two different stages, the energy transduction reaction (light stage) and the carbon fixation reaction (dark stage, or Calvin Cycle). During the light stage, two photosystems (PSI and PSII) are involved which are both located in the membranes of the chloroplast. Each photosystem contains an antenna complex responsible for capturing light energy, which is then passed to the reaction centre. Pigment molecules such as chlorophyll $a$ and $\mathrm{c}_{2}$, and peridinin that convert light energy into chemical energy are located in the peripheral antennae. Photosynthetically active radiation (PAR; $400-700 \mathrm{~nm}$ of solar radiation) first excites the reaction centre in PSII, causing electrons to pass down the electron transport chain via carriers like plastoquinone and plastocyanin to PSI. This electron transport is coupled to a proton transport across the thylakoid membrane, creating a proton gradient used by adenosine triphosphate (ATP)-synthase to generate ATP (Falkowski and Raven 2007). Electrons are replaced by splitting water molecules into protons, electrons and oxygen gas molecules. Reaction centres in PSI are also excited, passing electrons to ferredoxin and nicotinamide adenine dinucleotide phosphate (NADPH). Both ATP and NADPH are then used to provide energy and reductant for the fixation of $\mathrm{CO}_{2}$ in the Calvin Benson Cycle (Falkowski and Raven 2007).

Three possible sites of initial damage during bleaching have been proposed (Fig. 1.4). All potentially prohibit repair of photodamaged reaction centres of PSII and accelerate photoinhibition (Hill et al. 2004). In the process, reactive oxygen species (ROS) such as hydrogen peroxide $\left(\mathrm{H}_{2} \mathrm{O}_{2}\right)$, superoxide $\left(\mathrm{O}_{2}{ }^{-}\right)$, singlet oxygen $\left({ }^{1} \mathrm{O}_{2}\right)$ and highly reduced hydroxyl radical $\left(\mathrm{OH}^{*}\right)$ are being produced (see section 1.4 for more information). These can damage lipids, proteins and DNA and their production is prevalent in the marine environment (Lesser 2006, Venn et al. 2008). 


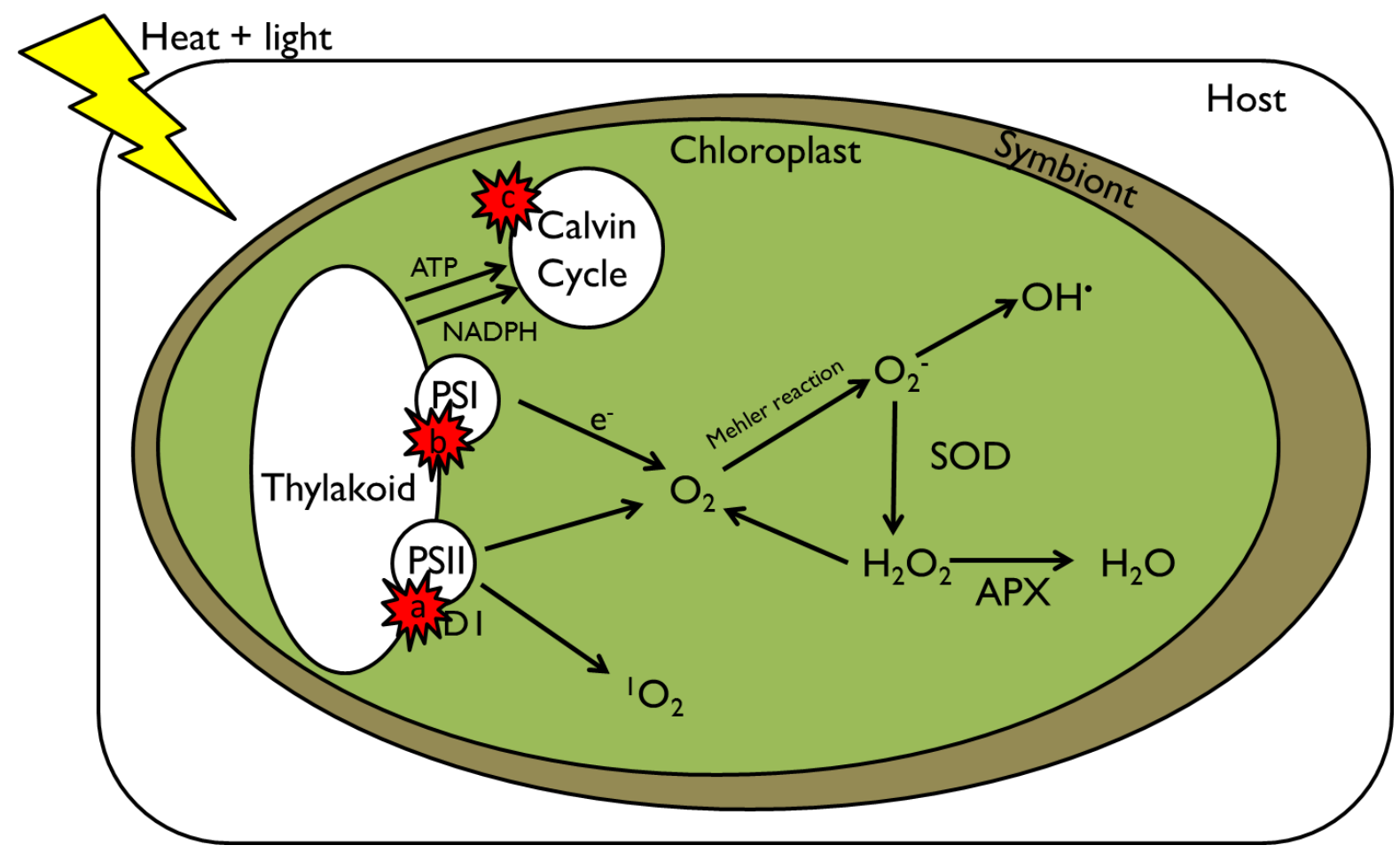

Figure 1.4: The three proposed sites (a-c) of initial damage in the photosystems of symbiotic dinoflagellates in a coral host due to temperature and light. a: Damage of PSII, including the D1 protein. b: Energetic uncoupling in the thylakoid membranes. c: Impairment of the Calvin cycle. Production of Reactive Oxygen Species (ROS) such as hydrogen peroxide $\left(\mathrm{H}_{2} \mathrm{O}_{2}\right)$, superoxide $\left(\mathrm{O}_{2}{ }^{-}\right)$, singlet oxygen $\left({ }^{1} \mathrm{O}_{2}\right)$ and the hydroxyl radical $\left(\mathrm{OH}^{*}\right)$ is counteracted by antioxidants superoxide dismutase (SOD) and ascorbate peroxidase (APX). Figure is adapted from Venn et al. (2008).

One possible site of damage is the D1 protein, which plays an important structural and functional role by binding components of the reaction centre and the electron transport chain together (Douglas 2003). Due to a high protein turnover, D1 is normally repaired at the same rate as damage occurs. Recently, it has become clearer that instead of damaging the D1 protein directly, thermal stress conditions might rather inhibit the D1 repair mechanism, i.e. protein re-synthesis and replacement (Nishiyama et al. 2006, Murata et al. 2007, Takahashi and Murata 2008). The inhibition leads to a loss of PSII functional reaction centres and explains the loss of PSII activity during stress (Warner et al. 1999, Lesser and Farrell 2004). 
Heat and light can also directly damage the thylakoid membranes of chloroplasts (Tchernov et al. 2004). Electron transport becomes energetically uncoupled and the transmembrane proton gradient, established by the photochemical reactions in the reaction centre, dissipates without generating ATP (Venn et al. 2008). Thus, pathways that facilitate build up of a proton gradient used to dissipate excess excitation energy are influenced or even inhibited (see section 1.5.1). The water-splitting complex remains active and the produced oxygen is reduced in the Mehler reaction to highly reactive superoxide $\left(\mathrm{O}_{2}^{-}\right)$by excess electrons derived from PSI (Fig. 1.5; Badger et al. 2000, Makino et al. 2002, Tchernov et al. 2004). Superoxide can be converted to the less reactive, but still damaging, hydrogen peroxide $\left(\mathrm{H}_{2} \mathrm{O}_{2}\right)$ by superoxide dismutase (Veljović-Jovanović 1998, Asada 2000). $\mathrm{H}_{2} \mathrm{O}_{2}$ can react with ferrous iron $\left(\mathrm{Fe}^{2+}\right)$ to form the most reactive ROS, a hydroxyl radical $\left(\mathrm{OH}^{*}\right)$ via the Haber-Weiss or Fenton reaction (Niyogi 1999, Weis 2008), but ideally it is reduced to water using ascorbate as the electron donor in the water-water cycle (Asada 2000). This way, the highly reactive oxygen species are effectively detoxified and the water-water cycle is an important mechanism for dissipating excess energy (Mullineaux et al. 2000). Thermal stress susceptibility of thylakoid membranes seems to be mainly determined by their lipid composition, i.e. saturation of the fatty acids (Tchernov et al. 2004).

A third site of initial damage, an impairment of the Calvin-Benson cycle caused by decreased carboxylation of ribulose-1,5-biphosphate by Rubisco has been proposed (Jones et al. 1998). Reduced consumption rates of products of electron transport (ATP, NADPH; referred to as sink limitation) restrict the flow rate in the electron transport chain and result in a maximal reduction of the plastoquinone pool. The subsequent build-up of excess energy leads to the formation of highly reactive triplet chlorophyll. The reactive chlorophyll molecule itself can then react with $\mathrm{O}_{2}$ to form the highly reactive singlet oxygen ( ${ }^{1} \mathrm{O}_{2}$; Veljović-Jovanović 1998, Apel and Hirt 2004, Hideg et al. 2007). If ${ }^{1} \mathrm{O}_{2}$ is not quenched by antioxidants $\beta$-carotene and $\alpha$-tocopherol, it can damage and react with the D1 protein and lead to bleaching of pigments in the photosynthetic apparatus of the thylakoids (Venn et al. 2008). Maximal reduction of the PSII quinone receptors can be prevented by the Mehler reaction as an alternative sink for electrons, but at the cost of producing reactive oxygen species (ROS; Badger et al. 2000). At the same time, ${ }^{1} \mathrm{O}_{2}$ can trigger the up-regulation of genes that are involved in the molecular defence against photo- 


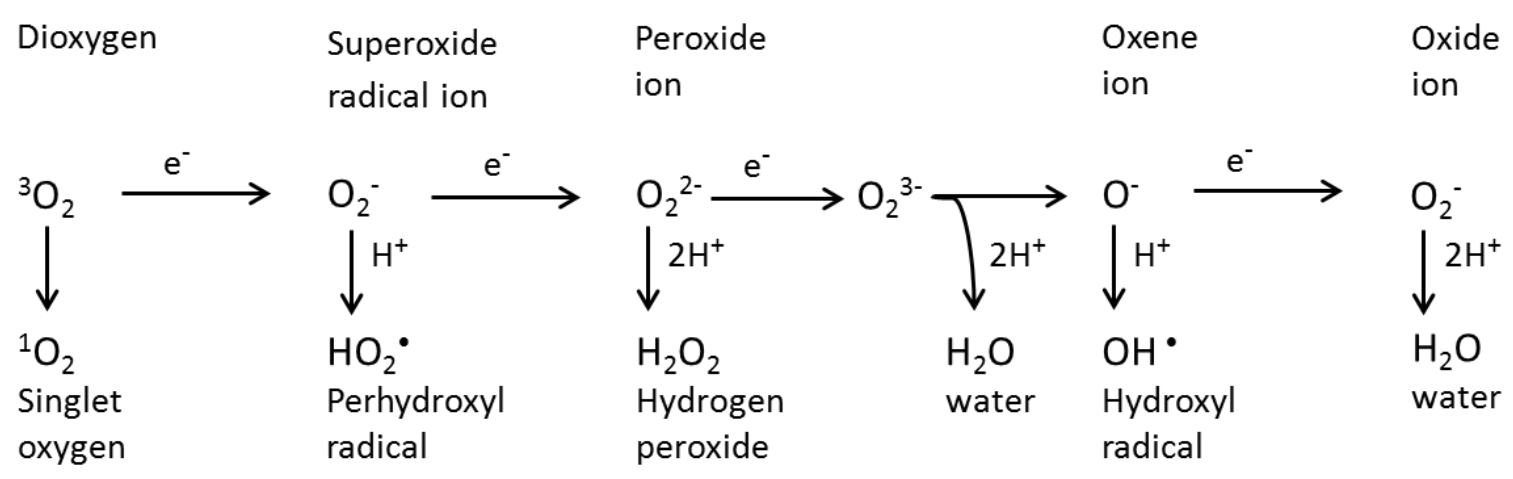

Figure 1.5: Generation of different reactive oxygen species (ROS) by energy transfer or sequential univalent reduction of ground state triplet oxygen (reproduced from Apel and Hirt 2004)

oxidative stress, for instance the glutathione peroxidase homologous gene (Leisinger et al. 2001, Op den Camp et al. 2003, Krieger-Liszkay 2004). Very recently, Buxton et al. (2011) provided evidence in support of this site of damage. By using methyl viologen, which accepts electrons from PSI and oxidizes ferredoxin, they were able to identify the initial site of damage as downstream from the light-dependent reactions in several Symbiodinium types (Buxton et al. 2011). However, they also concluded that the primary site of damage differed between Symbiodinium clades and possibly types.

\subsection{Oxidative stress and Reactive Oxygen Species (ROS)}

In recent years, it has become apparent that ROS are not only toxic by-products of aerobic metabolism, but a continuous product of cellular activity in plant chloroplasts, mitochondria, and peroxisomes (Mullineaux et al. 2000, Mittler 2002, Apel and Hirt 2004). ROS are used as signalling molecules to control processes such as programmed cell death, abiotic stress responses, pathogen defence and systemic signalling. During stress, ROS activate stress-response and defence pathways, and therefore can be viewed as secondary messengers (Mittler 2002, Wagner et al. 2005, Lesser 2006). It was even proposed by Foyer and Noctor (2005) that, instead of the highly negative term oxidative stress, the syndrome should be described as 'oxidative signalling'. However, in line with the majority of current literature, the term 'oxidative stress' will be retained here. 
Wherever the first site of thermally-induced stress might be (see previous section), it leads to the generation of ROS (Lesser 2006) such as hydrogen peroxide $\left(\mathrm{H}_{2} \mathrm{O}_{2}\right)$, singlet oxygen $\left({ }^{1} \mathrm{O}_{2}\right)$, superoxide $\left(\mathrm{O}_{2}{ }^{-}\right)$and the highly reduced hydroxyl radical $\left(\mathrm{OH}^{*}\right)$. If the production of ROS is not counterbalanced by antioxidants, a state of oxidative stress will develop inside the algal cells with possibly detrimental results (Sies 1997). ROS damage DNA, proteins and membranes (Lesser 1996, 1997) and are thought to trigger the expulsion of the symbiotic algae from the respective host (Tchernov et al. 2004, Smith et al. 2005). It is also proposed that ROS can diffuse from the algal cells into the host cells to cause more harm there (Downs et al. 2002, Flores-Ramírez and Liñán-Cabello 2007). Additionally, ROS are also produced directly by host mitochondria damaged by elevated temperatures (Nii and Muscatine 1997). Ultimately, when stress conditions persist, the continuous generation of ROS can lead to the coral's death (Richier et al. 2008).

Little is known about responses to oxidative stress at the cellular level in animals with photosynthetic symbionts (Venn et al. 2008). It has been shown that ROS play a central role in both responding to injuries to the partners and to inter-partner communication of a stress response (Weis 2008). ROS produced in the dinoflagellate symbiont through a photosynthetic dysfunction, initiate, together with reactive nitrogen species, a host-cell signalling cascade similar to an innate immune response that leads to the loss of the algae (Weis et al. 2008). In particular, the reactive nitrogen species nitric oxide (NO) may play an important role in the bleaching process, acting both as a cytotoxic and signalling molecule in animals (Weis 2008). In a study by Perez and Weis (2006), the symbiotic anemone Aiptasia pallida was exposed to heat stress or the photosynthetic inhibitor 3-(3,4dichlorophenyl)-1,1-dimethylurea (DCMU). Both treatments triggered the generation of NO, probably through a nuclear factor induced by ROS; moreover, the addition of NO to anemones at ambient temperature caused bleaching. Whether NO originates in the host, symbiont or both, is unclear, though NO was found in high concentrations in cultured and freshly isolated dinoflagellates at elevated temperature and subsequently in symbionts associated with bleaching corals (Trapido-Rosenthal et al. 2001, 2005, Bouchard and Yamasaki 2008). All of these studies provide evidence that NO, like ROS, might be a signalling molecule between the symbiotic partners (Weis 2008). 


\subsection{Protective mechanisms}

Most studies about coral bleaching have focused on the role of the symbiont in the bleaching process. As discussed earlier, different Symbiodinium types vary in their responses to temperature stress and light (Iglesias-Prieto et al. 2004). Some types are more susceptible to photoinhibition, which might lead to oxidative stress with possible spillover into the host, and expulsion and possibly permanent damage to the holobiont as a consequence (Downs et al. 2002, Flores-Ramírez and Liñán-Cabello 2007; Fig. 1.6). Other types are more tolerant and experience no photoinhibition under the same conditions. However, the host is not completely reliant on its symbiont's defence system and differences in the holobiont's stress response cannot be explained by the algal cell's susceptibilities alone. Rather, both symbiotic partners have developed strategies to deal with and possibly acclimatize to thermal and oxidative stressors (Middlebrook et al. 2008), and provide protection for each other (Bhagooli et al. 2008).

\subsubsection{Role of the symbiont}

Many studies have explored the role of the symbiont during bleaching events (e.g. Baker et al. 2004, Warner et al. 2006, Hill and Ralph 2008, Suggett et al. 2008). The earlier

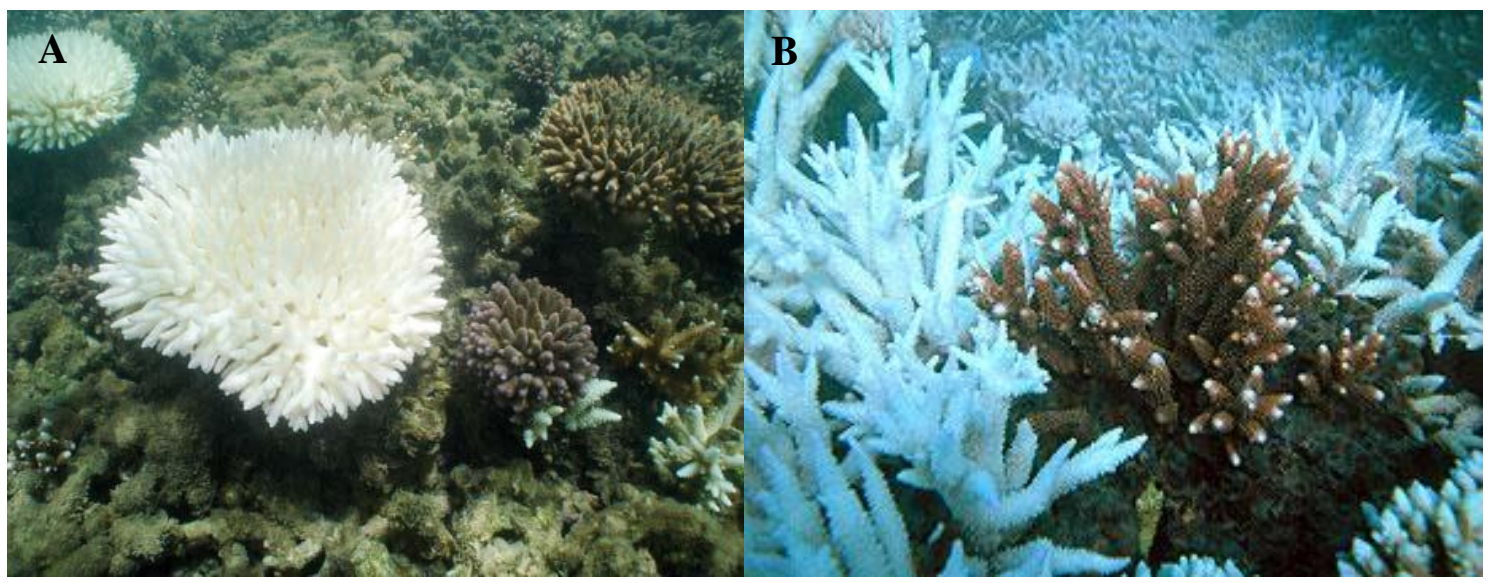

Figure 1.6: Differential bleaching by colonies of the same coral species. (A) $100 \%$ bleached Acropora millepora colonies dominated by Symbiodinium clade C2 and unbleached colonies with Symbiodinium clade D (photo: Jones et al. 2008); (B) the same Acropora species with bleached and unbleached individual colonies (photo: Hoegh-Guldberg 2006). 
discussed immense diversity of Symbiodinium types provides a wide array of stress responses which is influenced in several ways.

Non-photochemical quenching (NPQ) is one way of protecting the symbiosis from thermal damage (Müller et al. 2001). In this process, after absorbing light, the singlet-state excitation of a chlorophyll $a$ molecule is returned to ground state and excess photosynthetic energy is dissipated as heat (Hill et al. 2005). In a study by Gorbunov et al. (2001), it was shown that on a sunny day in shallow water, the integrated flux of photons absorbed and subsequently dissipated as heat, can be about four times that used for photosynthesis. A surplus of sunlight that exceeds the alga's capacity for $\mathrm{CO}_{2}$ fixation leads to a build-up of a gradient in $\mathrm{pH}(\Delta \mathrm{pH})$ across the thylakoid membrane that is produced by photosynthetic electron transport, with a decrease of the $\mathrm{pH}$ within the lumen. The decrease in lumen $\mathrm{pH}$ induces NPQ via protonation of PSII proteins and activation of the xanthophyll cycle. In the xanthophyll cycle, the carotenoid pigment pool increases, as does the conversion rate of the pigments diadinoxanthin to diatoxanthin (Dove et al. 2006). This xanthophyll pool may also increase with 'experience'- when the algal cells are more often subjected to high light intensities (Brown et al. 2002, Krämer et al. 2012). In a comparable study of the green alga Chlamydomonas reinhardtii, a deficiency in xanthophylls led to a loss in viability and photosynthetic activity along with destruction of the thylakoid membrane protein-pigment complexes, and an increase of ROS and membrane lipid peroxides (Baroli et al. 2004).

The symbiont also shares some protective mechanisms with the host which include the production of mycosporine-like amino acids (MAAs) and a range of antioxidants (see sections 1.5 .3 and 1.6 respectively for more information). All these mechanisms can vary between Symbiodinium types and have an effect on the symbiont's, and as a consequence, the holobiont's stress tolerance.

It is therefore an advantage for coral species to be able to exchange their dominant symbiont type for a genetically different assemblage when facing unusually high seawater temperatures (Baker et al. 2004). This is referred to as 'shuffling', when there is an increase in the relative abundance of a type already present in the coral's tissues in lower 
Chapter 1

numbers (Berkelmans and Van Oppen 2006, Abrego et al. 2008). Alternatively, 'switching' refers to a wholesale change in the symbiont type present as a result of a novel infection from the seawater (Buddemeier and Fautin 1993, Fautin and Buddemeier 2004) though evidence for this in situ is currently lacking. In both cases, the old symbiont community is replaced with one that is less susceptible to thermal stress and hence bleaching (Lesser 2007, Mostafavi et al. 2007, Jones et al. 2008). At the same time, the main source of ROS is eliminated from the host's cells (Merle et al. 2007, Obura 2008). Of particular note, many Symbiodinium types belonging to clade D have been described as being relatively thermally tolerant and often become dominant on reefs after bleaching events (Baker et al. 2004, Rowan 2004, Jones et al. 2008).

\subsubsection{Role of the host}

The role of the cnidarian host in protecting the holobiont from stress has been widely overlooked in the past. Recently, some studies have emerged that placed more emphasis on how the host influences the bleaching response (Bhagooli et al. 2008, Baird et al. 2009). Several potential protective mechanisms that are either unique to the host or can be provided by both symbiotic partners exist and suggest that host and symbiont should be treated equally.

Up to $97 \%$ of the coral hosts on a reef contain a range of fluorescent proteins (FPs), which belong to a single family of proteins closely related to the green fluorescent proteins (Baird et al. 2009). FPs absorb, scatter and dissipate high-energy solar radiation via fluorescence, at wavelengths of low photosynthetic activity (Salih et al. 2000). They thereby reduce photoinhibition and play a photoprotective role. Furthermore, FPs also have very high $\mathrm{H}_{2} \mathrm{O}_{2}$ scavenging activities (Palmer et al. 2009, 2010). High concentrations of FPs and therefore high fluorescence are found in more bleaching-tolerant coral taxa, while more bleaching- susceptible coral species have relatively low concentrations of FPs (Baird et al. 2009).

Some coral species can also adjust their diet as part of their bleaching protection. By increasing their heterotrophic food intake, they are better equipped to survive bleaching 
than taxa that solely rely on autotrophy. Moreover, this change in behaviour will decrease the energetic demands on the symbionts, which are then able to put extra energy into their own defence systems (Borell and Bischof 2008, Baird et al. 2009).

\subsubsection{Joint roles}

While the coral host and algal symbiont have some unique protective mechanisms as discussed before, they appear to also produce some similar chemical compounds when faced with stress.

Mycosporine-like amino acids (MAAs) that absorb ultraviolet radiation (UV) and dissipate UV energy, act as a natural sunscreen in the coral- algal symbiosis. Traditionally, it was assumed that MAAs can be produced only by the symbiont as animals lack the necessary pathways for their synthesis, though this view has been challenged recently (Starcevic et al. 2010, Shinzato et al. 2011). Still, the abundance and diversity of MAAs in host tissue and the holobiont, respectively, have been found to be much higher than in freshly isolated symbionts (Shick and Dunlap 2002). Reasons for these patterns could be either host stimulation of the dinoflagellate symbiont to produce a larger range of MAAs in hospite, or modification of MAAs translocated from the symbiont to the host. Either way, it shows that the host influences the diversity as well as the distribution of MAAs, and that it could possibly exert control over the amount of damage sustained by the symbiont cell (FerrierPagès et al. 2007, Baird et al. 2009). It appears that the generation of a first set of MAAs is followed by a later increase in different MAAs. This suggests a precursor-product relationship between the primary and secondary MAAs. It should be noted though, that while UV probably acts as a signal to start primary MAA synthesis, secondary MAAs continue to be generated after UV stress has stopped (Shick 2004).

Additionally, both symbiotic partners can produce certain proteins to handle stressful situations. When exposed briefly to relatively high temperatures, the holobiont can synthesize heat-shock proteins (Hsps) that correlate with the acquisition of enhanced thermotolerance (Black et al. 1995, Leggat et al. 2007). Hsps are not exclusively produced during thermal stress but act as molecular chaperones which maintain protein structure and 
cell function following different stressors. A number of different Hsps which are mostly produced in three different molecular weight classes (small Hsps: 18-30 kDa, Hsp70: 68-74 kDa, Hsp90: 80-90 kDa) are found in coral tissue (Black et al. 1995) and areas of higher concentrations of Hsps appear to bleach less (Baird et al. 2009).

While not a mechanism per se, how and to what extent different coral-dinoflagellate symbioses react to exogenous stress depends not only on their specific resistance but also on their capacity to recover from bleaching and possible acclimation, i.e. experiencemediated increase in resistance to bleaching (Visram and Douglas 2007). This is the case in corals that inhabit more variable habitats, like shallow reef pools, where they possess a substantially higher thermal tolerance that cannot be explained by heat-resistant symbionts alone (Barshis et al. 2010, Oliver and Palumbi 2011). Short-term and recent history, such as that related to seasonal change, can also play a role in a coral's stress response. There has been evidence that corals that have experienced stresses such as increased temperature in the past are better able to deal with them in the future (Maynard et al. 2008, Putnam and Edmunds 2010), for instance by increasing their antioxidant capacity (Griffin et al. 2006).

Antioxidant production as a form of oxidative stress resistance, both in the cnidarian host as well as the dinoflagellate symbiont is very important and will be discussed in more depth in the next section (section 1.6).

\subsection{Antioxidants}

The production of antioxidants by both partners also plays an important, but somewhat understudied role in the fight against oxidative stress. Antioxidants are defined as 'any substance that, when present at low concentrations compared with those of an oxidizable substrate, significantly delays or prevents oxidation of that substrate' (Griffin and Bhagooli 2004). Several earlier studies have suggested increased antioxidant activity in corals under oxidative stress conditions (Downs et al. 2002, Liñán-Cabello et al. 2009). 


\subsubsection{Enzymatic antioxidants}

Evidence of enzymatic antioxidant activity has been found in several coral studies (e.g. Downs et al. 2002, Sigaud-Kutner et al. 2002, Griffin and Bhagooli 2004, Higuchi et al. 2008) and a comparison between symbiotic and aposymbiotic sea anemones showed great differences in their antioxidant responses to oxidative stress (Richier et al. 2005), suggesting that symbiotic cells adapt to stress, while non-symbiotic cells remain acutely sensitive.

Enzymatic antioxidants include superoxide dismutase (SOD) that reduces superoxide to hydrogen peroxide $\left(\mathrm{H}_{2} \mathrm{O}_{2}\right)$, which is then removed by catalase (CAT) and ascorbate peroxidase (APX) and reduced to water and oxygen (Venn et al. 2008). Both host and symbiont produce SOD, however several forms of this antioxidant exist: both symbiotic partners contain copper and zinc superoxide dismutase $(\mathrm{Cu} / \mathrm{ZnSOD})$ and manganese superoxide dismutase (MnSOD), while Symbiodinium cells also possess iron superoxide dismutase (FeSOD; Okamoto et al. 2001, Richier et al. 2003, Plantivaux et al. 2004, Lesser 2006). CAT ( $\mu \mathrm{M}$ range) and APX (mM range) on the other hand, show different affinities for $\mathrm{H}_{2} \mathrm{O}_{2}$, suggesting they belong to two different classes of $\mathrm{H}_{2} \mathrm{O}_{2}$ scavenging enzymes: while APX manages ROS on lower levels for signalling purposes, CAT might be responsible for the removal of excess ROS under stress conditions (Mittler 2002). A study with scleractinian hosts found increasing levels of these antioxidants with increasing temperatures, in both the hosts as well as in their symbiotic dinoflagellates, though the level of increase varied between different coral species and different antioxidant types (Griffin and Bhagooli 2004, Yakovleva et al. 2004). Light spectrum and length seem to have similar effects as temperature, with SOD and CAT levels in the holobiont being shown to follow the diurnal light cycle, though higher levels of antioxidants were found in the host than the symbiont, probably due to accumulation of free radicals in the coral tissue (Levy et al. 2006a, b). CAT activity also seems to be lower in freshly isolated symbiotic dinoflagellates than in cultured ones, indicating that inside host cells the algae also rely on the host's antioxidant capacity (Lesser and Shick 1989a, b, Merle et al. 2007). Consistent with this, Nii and Muscatine (1997) showed that oxidative stress in Aiptasia pulchella mainly produces a host response and that freshly isolated symbionts hardly produce any SOD in response to elevated temperatures. SOD, CAT and APX act together to inactivate surplus ROS and prevent the formation of the $\mathrm{OH}^{-}$generated from $\mathrm{O}_{2}{ }^{-}$and $\mathrm{H}_{2} \mathrm{O}_{2}$ in the 
presence of transmition metals (e.g., ferric iron), via the Haber-Weis or Fenton reactions (Lesser et al. 1990, Lesser 1997). Antioxidants are therefore important tools for corals and their algal symbionts to lessen the effects of oxidative stress induced by climate change (Downs et al. 2000, Merle et al. 2007).

\subsubsection{Non-enzymatic antioxidants}

There are also a range of non-enzymatic antioxidants that help to neutralize ROS and minimize intracellular oxidative stress.

Two of the major non-enzymatic antioxidants are glutathione (GSH) and ascorbate which are connected by the ascorbate-glutathione cycle. By reacting with different ROS such as ${ }^{1} \mathrm{O}_{2}, \mathrm{O}_{2}{ }^{-}$and $\mathrm{OH}^{\bullet}$, GSH forms a thiyl radical that reacts with a second oxidized glutathione (GSSG), forming a disulphide bond when oxidized (Halliwell and Gutteridge 1990); the ratio of GSH to GSSG can be used as an indicator of oxidative stress in cells. Ascorbate, on the other hand, is oxidized to monodehydroascorbate (MDA) and dehydroascorbate (DHA), and GSSG, MDA, and DHA can be reduced again to reform GSH and ascorbate through the aforementioned ascorbate-glutathione cycle. Decreased ascorbate levels and altered GSH content in cells are therefore signs for hypersensitivity to stress (Apel and Hirt 2004).

Carotenoid antioxidants in contrast, play a role in the xanthophyll cycle where excess energy is dissipated as heat (Lesser 2006), as discussed in section 1.5. These nonenzymatic antioxidants function as accessory pigments in light harvesting, and also quench ROS and prevent lipid peroxidation (Fridovich 1998). They are lipid soluble antioxidants that are synthesized by photoautotrophic organisms and can potentially be taken up heterotrophically by the coral host (Mobley and Gleason 2003).

There are also small molecule antioxidants worth noting, such as dimethylsulfide (DMS) that is synthesised from dimethylsulphoniopropionate (DMSP) by many marine macrophytes and phytoplanktonic organisms (Steinke et al. 2011). DMS and DMSP can both quench $\mathrm{OH}^{*}$, and the ability of DMS to diffuse through biological membranes and act 
in any cellular compartment makes it an especially effective antioxidant (Sunda et al. 2002, Lesser 2006).

The previous sections have given an overview of the events that occur in the cnidariandinoflagellate symbiosis during stress, such as bleaching events induced by climate change. Thermal stress causes damage in the algal photosynthetic apparatus and leads to the formation of various reactive oxygen species (ROS) and intracellular oxidative stress (Venn et al. 2008). Both symbiotic partners have developed different strategies to deal with stress, including the generation of antioxidants (Lesser 2006) and many studies have shown that stress responses vary between even closely related Symbiodinium types (Thornhill et al. 2007, Sampayo et al. 2008). Unlike in the wild, where both coral host and algal symbiont will contribute to health and survival of the association (Gates et al. 1995, Yellowlees et al. 2008, Baird et al. 2009), the effects of various environmental factors on the algal cell can be isolated using Symbiodinium cultures. This is useful when trying to define properties of stress-resistance in specific sub-clades, independent from the host's influence. In particular, it may give a clearer picture of how different types deal with stress, and the influence it has on different parts of the photosynthetic apparatus and cell components such as membranes, proteins and nucleic acids (Lesser 1996, 1997, Robison and Warner 2006). At the same time, it is important to remember that in the wild, both coral host and algal symbiont will contribute to health and survival of the association and that a realistic picture of events can only be maintained by studying the holobiont.

\subsection{Aim of the study}

The aim of this study was to explore if the susceptibilities to oxidative stress of a range of Symbiodinium clades and types (i.e. sub-clades) in different states of symbiosis (i.e. in hospite, freshly isolated and in culture) and from different geographic origins are related to their thermal susceptibilities, and if the response to oxidative and thermal stress is a function of the dinoflagellate's antioxidant activity. 
Chapter 1

The specific objectives were to:

1) Establish whether distinct types of Symbiodinium cultures have different sensitivities to oxidative stress, and whether these are related to their thermal sensitivities.

This was achieved by exposing a range of Symbiodinium types to thermal and oxidative stress, and measuring their photosynthetic performance. It was hypothesized that a range of responses would be observed, and that the more thermally sensitive types would also be the most susceptible to oxidative stress.

2) Measure the activity and relative amounts of specific ROS in different types of cultured Symbiodinium in response to thermal and hydrogen peroxide $\left(\mathrm{H}_{2} \mathrm{O}_{2}\right)$ induced oxidative stress.

This was achieved by using fluorescent probes, flow cytometry and confocal microscopy. It was hypothesized that distinct Symbiodinium types would produce different types of ROS and/or different amounts of these specific ROS between stressors.

3) Measure total antioxidant activity in different cultured Symbiodinium types under oxidative stress.

Total antioxidant activity was measured using the Ferric Reducing Antioxidant Potential (FRAP) assay and the Cellular Antioxidant Assay (CAA); results for both assays were compared. It was hypothesized that antioxidant production would increase with increasing oxidative stress, but that it would differ between Symbiodinium types, with a lesser potential to produce antioxidants in those types that are more sensitive to stress.

4) Compare and contrast the responses of distinct Symbiodinium types to thermal and oxidative stress when both in hospite and freshly isolated from corals

Photosynthetic efficiency under stress and subsequent antioxidant production were compared between different Symbiodinium types when in hospite and freshly isolated from corals (including the same Symbiodinium types in different host species). It was hypothesized that the same Symbiodinium types would behave similarly, irrespective of host origin and symbiotic state 
Chapter 1 


\section{Chapter 2: Variability in thermal and oxidative stress response in distinct Symbiodinium cultures}

\subsection{Introduction}

The success of scleractinian corals as modern reef-builders is dependent on the symbiosis between these corals and their dinoflagellate symbionts of the genus Symbiodinium, as the presence of the symbionts contributes significantly to the productivity, survival and success of their hosts (Hoegh-Guldberg et al. 2007). Symbiodinium cells occur inside the host's gastrodermal cells where they supplement the coral's metabolism through the provision of photosynthetic compounds; in return they receive inorganic nutrients (e.g. nitrogen and phosphorus) from the host (see reviews by Yellowlees et al. 2008, Davy et al. 2012 and references therein). The genus Symbiodinium is very diverse with nine clades and many sub-clades ('types'; LaJeunesse 2001, Coffroth and Santos 2005, Pochon and Gates 2010). Even closely related types can differ in their physiological responses to environmental factors or host specificity, preventing generalizations about the characteristics of each clade. Symbiont distributions vary; some algal taxa are associated with widely different host species and occur across diverse geographic regions, while others show higher host specificity or appear regionally endemic (LaJeunesse 2001).

The steady state of an established cnidarian-dinoflagellate symbiosis can be radically altered by even subtle changes in abiotic factors influencing either partner's physiology (Fitt 2000). In recent years, this has occurred more frequently in so-called coral bleaching events (Hoegh-Guldberg et al. 2007). When the holobiont encounters exogenous stress, the density of algal symbionts or concentrations of their photosynthetic pigments inside the host are reduced, the coral loses its colour and the underlying calcium carbonate skeleton becomes apparent (Gates et al. 1992, Douglas 2003, Weis 2008). Bleaching is predominately attributed to higher than normal seawater temperatures (Weis 2008, Lough and Van Oppen 2009, Lesser 2011), the likely consequence of climate change, in combination with high light intensities (Hill and Ralph 2007, Ainsworth et al. 2008). As corals and other tropical symbioses with Symbiodinium spp. live in environments that approach their upper thermal limits, an increase of just a few degrees Celsius can cause 
bleaching (Fitt et al. 2001). This results in reduced growth, increased susceptibility to disease and dramatically increased mortality of the symbiotic partners (Hoegh-Guldberg et al. 2007, Weis 2008). While the underlying cellular mechanisms responsible for bleaching have yet to be fully identified (Weis 2008, Weis et al. 2008, Davy et al. 2012), it has been suggested that higher temperatures primarily cause a negative impact on the light reactions of photosynthesis (Venn et al. 2008) with sites of damage being the thylakoid membranes of chloroplasts (Tchernov et al. 2004, Venn et al. 2008) and inhibition of the D1 protein repair mechanisms (Douglas 2003, Murata et al. 2007, Takahashi and Murata 2008). But heat and light can also impair the Calvin-Benson cycle which leads to a build-up of excess energy in PSII (Jones et al. 1998).

If damage is not repaired and the generation of reactive oxygen species (ROS) exceeds their detoxification, photoinhibition can result (Lesser 2006). ROS such as hydrogen peroxide $\left(\mathrm{H}_{2} \mathrm{O}_{2}\right)$, superoxide $\left(\mathrm{O}_{2}^{-}\right)$, singlet oxygen $\left({ }^{1} \mathrm{O}_{2}\right)$ and the highly reactive hydroxyl radical $\left(\mathrm{OH}^{\circ}\right)$ damage DNA, proteins and membranes (Lesser 1996, 1997). The highly cellpermeable $\mathrm{H}_{2} \mathrm{O}_{2}$ is also able to diffuse from algal to host cells and trigger the expulsion of the alga from the host cell (Tchernov et al. 2004, Smith et al. 2005, Weis 2008). ROS are normally neutralised by antioxidant compounds such as the enzyme superoxide dismutase (SOD) which reduces $\mathrm{O}_{2}{ }^{-}$to $\mathrm{H}_{2} \mathrm{O}_{2}$; this is subsequently reduced by ascorbate peroxidase (APX) and catalase (CAT) to water and oxygen (Venn et al. 2008). During protracted abiotic stress, however, the antioxidant system can be overwhelmed, leading to a net increase of ROS and oxidative stress inside the algal cells. Given the differential susceptibilities of corals to thermal stress, it is therefore possible that different Symbiodinium types display dissimilar responses to oxidative stress with, for example, thermally sensitive corals containing symbionts that are especially sensitive to ROS (Fisher 2006).

In this baseline study, I compared the effects of thermal and oxidative stress (induced by $\mathrm{H}_{2} \mathrm{O}_{2}$ ) on the photo-physiology of different Symbiodinium types. Symbiodinium cultures and freshly isolated Symbiodinium cells were used that represented a range of clades and geographical origins (i.e. tropical, sub-tropical and temperate) and type-specific susceptibilities to stress were predicted. Given the close relationship between thermal and 
Chapter 2

oxidative stress (Lesser 2006), it was expected that Symbiodinium types with a tolerance of high temperature would be more tolerant of oxidative stress than types with a greater sensitivity to high temperature.

\subsection{Material and Methods}

\subsubsection{Symbiodinium cultures}

Experiments used six Symbiodinium- cultures originally isolated from a range of cnidarian host species and geographic regions (A1, B2, E1, F1, S. californium; Table 2.1), and two types freshly isolated from two anemone species (A1, B1). Throughout this thesis, Symbiodinium types, or in one case the species name, are used for culture identification (i.e. type A1, B2, S. californium), with additional geographic information to differentiate more than one culture of a distinct type; i.e. types E1-O from Okinawa, F1-H from Hawaii, F1-P from an unknown location in the Pacific. Freshly isolated Symbiodinium types are identified by the suffix 'FIS' (i.e. A1-FIS, B1-FIS).

Cultures were maintained at $26.5 \pm 1{ }^{\circ} \mathrm{C}$ on a $12 \mathrm{~h}$ light: $12 \mathrm{~h}$ dark cycle for a minimum of one year before use. White light at $55 \pm 5 \mu \mathrm{mol}$ photons $\mathrm{m}^{-2} \mathrm{~s}^{-1}$ was supplied by two Phillip TLD $36 \mathrm{~W}$ fluorescent tubes. Cultures were maintained in silica-free f/2 medium (NCMA, East Boothbay, Maine, USA) and sub-cultured every 6-8 weeks. All experiments were conducted 4-6 weeks after sub-culturing.

\subsubsection{Genotyping of Symbiodinium cultures}

For DNA extraction, Symbiodinium cells were incubated in guanidinium solution

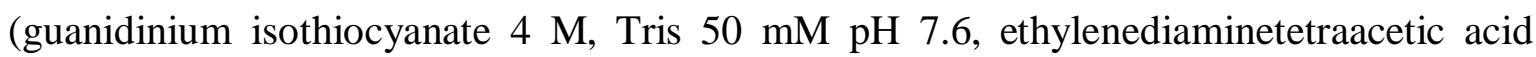
(EDTA) $10 \mathrm{mM}$; $0.02 \%$ sarkosyl (w/v), 0.01\% b-mercaptoethanol (v/v) for five days at $4{ }^{\circ} \mathrm{C}$. DNA extraction followed the method of Stat et al. (2009). Resulting DNA was used in conjunction with Symbiodinium specific ITS2 primers $(10 \mathrm{mM}$; forward primer: GTG AAT TGC AGA ACT CCG TG; reverse primer: CCT CCG CTT ACT TAT ATG CTT; 
Table 2.1: Symbiodinium cultures and freshly isolated Symbiodinium with corresponding host species, geographic origin and sub-clade.

\begin{tabular}{|c|c|c|c|c|}
\hline Culture I.D. & $\begin{array}{l}\text { Origin host } \\
\text { species }\end{array}$ & $\begin{array}{l}\text { Geographic origin } \\
\text { (approx. coordinates) }\end{array}$ & $\begin{array}{l}\text { Temperature } \\
\text { range* }\end{array}$ & $\begin{array}{l}\text { Sub- } \\
\text { clade }\end{array}$ \\
\hline CCMP2467 & $\begin{array}{l}\text { Stylophora } \\
\text { pistillata }\end{array}$ & $\begin{array}{l}\text { Gulf of Aqaba }(29.00 \mathrm{~N} \\
34.75 \mathrm{E})\end{array}$ & $20.5-27^{\circ} \mathrm{C}$ & A1 \\
\hline CCMP2459 & Oculina diffusa & $\begin{array}{l}\text { Bermuda }(32.38 \mathrm{~N} \\
64.68 \mathrm{~W})\end{array}$ & $18-29{ }^{\circ} \mathrm{C}$ & B2 \\
\hline A001 & Acropora sp. & Okinawa (26.3N 127.56E) & $21-30{ }^{\circ} \mathrm{C}$ & E1 \\
\hline Mv & $\begin{array}{l}\text { Montipora } \\
\text { verrucosa }\end{array}$ & Hawaii (21.26N $157.47 \mathrm{~W})$ & $24-27{ }^{\circ} \mathrm{C}$ & $\mathrm{F} 1$ \\
\hline AiptasiaI & Aiptasia pulchella & $\begin{array}{l}\text { Pacific (location } \\
\text { unknown) }\end{array}$ & & $\mathrm{F} 1$ \\
\hline S. californium & $\begin{array}{l}\text { Anthopleura } \\
\text { elegantissima }\end{array}$ & $\begin{array}{l}\text { Southern California } \\
(32.5 \mathrm{~N} 117.15 \mathrm{~W})\end{array}$ & $13.5-20^{\circ} \mathrm{C}$ & E1 \\
\hline N/A & $\begin{array}{l}\text { Anthopleura } \\
\text { aureoradiata }\end{array}$ & $\begin{array}{l}\text { Wellington, New Zealand } \\
(41.6 \mathrm{~S} 174.55 \mathrm{E})\end{array}$ & $7.9-19.6^{\circ} \mathrm{C}$ & A1 \\
\hline N/A & Aiptasia pulchella & $\begin{array}{l}\text { Pacific (location } \\
\text { unknown) }\end{array}$ & & B1 \\
\hline
\end{tabular}

* Sources: NOAA, Manasrah et al. 2006, Surf-forecast.com, Greater Wellington Regional Council

Logan et al 2010) in PCR reactions with a final volume of $25 \mu \mathrm{L}$. PCR reactions used MyTaqTM mastermix (Bioline, London, UK). The PCR program consisted of $3 \mathrm{~min}$ at $95{ }^{\circ} \mathrm{C}, 28$ cycles of $15 \mathrm{~s} 95^{\circ} \mathrm{C}, 15 \mathrm{~s} 57{ }^{\circ} \mathrm{C}, 10 \mathrm{~s} 72{ }^{\circ} \mathrm{C}$. Successful PCR was confirmed using a 1.5\% agarose gel (Sigma Aldrich New Zealand Ltd) run at $120 \mathrm{~V}$, for $45 \mathrm{~min}$. Gels were stained with ethidium bromide and visualised under UV light. PCR products were purified with Affymetrix USB ExoSAP-IT (In Vitro Technologies; Victoria, Australia) according to manufacturer's instructions and sent to Macrogen Inc. (Seoul, Korea) for sequencing. Sequences were analysed using the GeoSymbio Database, NCBI Genbank and Geneious software (Geneious 5.5.3; Biomatters Ltd. Auckland, NZ). 
Chapter 2

\subsubsection{Thermal stress experiment}

Symbiodinium cultures were centrifuged $(1800 \times g)$ for $5 \mathrm{~min}$ and then concentrated to $\sim 3 \times 10^{6}$ cells $\mathrm{mL}^{-1}$ in filtered seawater $(1 \mu \mathrm{m}-\mathrm{FSW})$; concentrations were estimated from haemocytometer counts ( $\times 100$ magnification; 6 replicates). Aliquots containing $300 \mu \mathrm{L}$ of algal suspensions were pipetted into black plastic 96 well-plates, $(\mathrm{n}=6$ per Symbiodinium type per plate). The photosynthetic performance of photosystem II (PSII) for each replicate suspension was estimated using imaging pulse amplitude modulated chlorophyll fluorometry (IPAM, M-Series, Maxi Version; Walz, Germany).

Each well plate was placed in water baths either at 27 (control), 30, 32 or $34{ }^{\circ} \mathrm{C}$ and illuminated at $100 \mu \mathrm{mol}$ photons $\mathrm{m}^{-2} \mathrm{~s}^{-1}$ using light banks of white $5 \mathrm{~W}$ halogen light supplied by Exe Dichro-Cool ${ }^{\mathrm{TM}}$. Further IPAM measurements were taken 1, 2 and $4 \mathrm{~h}$ after the initial measurement.

For each measurement, a saturation pulse (strength 8 , width: $0.8 \mathrm{sec}$ ) was applied to the samples after 20 min dark adaption for measurement of maximum quantum yield of PSII $\left(F_{\mathrm{v}} / \mathrm{F}_{\mathrm{m}}=\left(\mathrm{F}_{\mathrm{m}}-\mathrm{F}_{0}\right) / \mathrm{F}_{\mathrm{m}}\right)$. Values of $\mathrm{F}_{\mathrm{m}}$ (maximum fluorescence in the dark) and $\mathrm{F}_{0}$ (dark fluorescence yield) were calculated from a circular area of interest laid over the image of each well of the well plate. Additionally, samples were subjected to an irradiance ramp (rapid light curve, RLC) comprising of 11 steps. PAR increased from 1 to $466 \mu \mathrm{mol}$ photons $\mathrm{m}^{-2} \mathrm{~s}^{-1}$ and results were used to calculate maximum electron transport rate $\left(E R_{\max }\right)$ obtained by curve fitting (Platt et al. 1980, Ralph et al. 2002). As different sets of cells were used for $\mathrm{F}_{\mathrm{v}} / \mathrm{F}_{\mathrm{m}}$ and RLC measurements, NPQ could not be determined. Instead, the maximum excitation pressure over PSII $Q_{m}$ was calculated similarly to the equation of Iglesias-Prieto et al. (2004), using data from both the dark adapted samples as well as the RLC:

$$
\mathrm{Q}_{\mathrm{m}}=1-\left[\left(\Delta \mathrm{F} / \mathrm{F}_{\mathrm{m}} \text { at } 151 \mu \mathrm{mol} \text { photons } \mathrm{m}^{-2} \mathrm{~s}^{-1}\right) /\left(\mathrm{F}_{\mathrm{v}} / \mathrm{F}_{\mathrm{m}}\right)\right]
$$

The effective quantum yield of PSII $\left(\Delta F / F_{m}{ }^{\prime}=F_{m}{ }^{\prime}-F / F_{m}{ }^{\prime}\right)$ is calculated with $F_{m}{ }^{\prime}$ (maximum fluorescence in the light, here at $151 \mu \mathrm{mol}$ photons $\mathrm{m}^{-2} \mathrm{~s}^{-1}$ of RLC) and $\mathrm{F}$ (fluorescence yield in the light). 


\subsubsection{Oxidative stress experiment}

Aliquots containing $270 \mu \mathrm{L}$ Symbiodinium cell suspensions were pipetted into the wells of a black 96 well-plate, and $30 \mu \mathrm{L}$ of hydrogen peroxide $\left(\mathrm{H}_{2} \mathrm{O}_{2}\right)$ in FSW was added, to give final concentrations of 1,2 and $4 \mathrm{mM}$; a control contained no $\mathrm{H}_{2} \mathrm{O}_{2}$ but instead, a further $30 \mu \mathrm{L}$ of seawater was added to the cell suspensions $(\mathrm{n}=5$ per Symbiodinium type per $\mathrm{H}_{2} \mathrm{O}_{2}$ treatment). $\mathrm{H}_{2} \mathrm{O}_{2}$ concentrations were chosen as preliminary experiments showed that they elicited a full range of responses in Symbiodinium cells. The well-plates were placed in a water bath at $27{ }^{\circ} \mathrm{C}$ and illuminated as described in section 2.2.3. An initial IPAM measurement was performed at $30 \mathrm{~min}$ after dark adaption and 1, 2 and $4 \mathrm{~h}$ thereafter. Again, $\mathrm{F}_{\mathrm{v}} / \mathrm{F}_{\mathrm{m}}, \mathrm{ETR}_{\max }$ and $\mathrm{Q}_{\mathrm{m}}$ were measured.

At the same time as the IPAM measurements, $\mathrm{O}_{2}$ fluxes (respiratory consumption and photosynthetic production) were measured for each treatment. The same cell concentration as in the corresponding oxidative stress experiments was used $\left(\sim 3 \times 10^{6}\right.$ cells $\left.\mathrm{mL}^{-1}\right)$. Aliquots of $9 \mathrm{~mL}$ cell suspension were pipetted into plastic tubes to which $1 \mathrm{~mL}$ of $\mathrm{H}_{2} \mathrm{O}_{2}$ was added to give final concentrations of 1,2 and $4 \mathrm{mM}$; a control contained no $\mathrm{H}_{2} \mathrm{O}_{2}$ and instead, $1 \mathrm{~mL}$ of sea water was added ( $\mathrm{n}=5$ per Symbiodinium type per $\mathrm{H}_{2} \mathrm{O}_{2}$ treatment). Tubes were placed in a water bath at $27{ }^{\circ} \mathrm{C}$ and illuminated as described in section 2.2.3. After one hour, tubes were centrifuged $(1800 \times g)$ for $5 \mathrm{~min}$ to remove excess $\mathrm{O}_{2}$ produced during incubation with $\mathrm{H}_{2} \mathrm{O}_{2}$ that would otherwise have interfered with subsequent $\mathrm{O}_{2}$ flux measurements. Symbiodinium cells were re-suspended in $10 \mathrm{~mL}$ FSW and placed inside 10 $\mathrm{mL}$ glass chambers in a water bath at $27{ }^{\circ} \mathrm{C}$. Each glass chamber was then sealed with a stopper that contained an oxygen electrode (Fibox 3, PreSense Precision Sensing GmbH, Regensburg, Germany). Respiration rates in the dark as well as $\mathrm{O}_{2}$ development in the light (250 $\mu \mathrm{mol}$ photons $\mathrm{m}^{-2} \mathrm{~s}^{-1}$; white $5 \mathrm{~W}$ halogen light) of the Symbiodinium cells were measured whilst stirred by a magnetic stirrer (speed $120 \mathrm{rpm} ; \mathrm{H}+\mathrm{P}$ Labortechnik AG, Oberschleissheim, Germany). Each measurement was run until a clear trend emerged and $\mathrm{O}_{2}$ fluxes were measured and subsequently recorded by Oxyview PST3-V5.3.2 (respirometry software). One measurement was performed per treatment and replicate. Unlike the IPAM measurements, $\mathrm{O}_{2}$ fluxes were not compared over time due to the lengthy duration of the measurements. 
Chapter 2

\subsubsection{Statistical analysis}

Normality was tested using Shapiro Wilk's test and homogeneity of variance was tested by Levene's test. For normally distributed PAM data after transformation, several repeated measure analyses of variance (rANOVA; Symbiodinium type $\mathrm{x}_{2} \mathrm{O}_{2}$ concentration over time) with Greenhouse-Geisser correction were performed. If normality could not be achieved, the non-parametric Friedman Test was performed to test for any significant time effect. Subsequently, Mann-Whitney U Tests were performed for single time points to test for differences of means between treatments $(p \leq 0.05)$.

For respirometry measurements, data were transformed and subjected to a two-way ANOVA (Symbiodinium type $\mathrm{x}_{2} \mathrm{O}_{2}$ concentration). Subsequent post hoc tests were performed for PAM as well as respirometry data to test for differences of means (Tukey HSD, $\mathrm{p} \leq 0.05$ ). For all data analyses, PASW Statistics 18 was used (IBM SPSS, IBM Corp., Armonk, NY, USA).

\subsection{Results}

\subsubsection{Thermal stress experiment}

The maximum quantum yield of PSII $\left(\mathrm{F}_{\mathrm{v}} / \mathrm{F}_{\mathrm{m}}\right)$ changed with time in all Symbiodinium types in response to elevated temperature (Friedman Test, $\chi^{2}=219.1$, df $=3, \mathrm{p}<0.001$ ). Changes in $\mathrm{F}_{\mathrm{v}} / \mathrm{F}_{\mathrm{m}}$ were evident after only 80 min exposure between the control temperature and $34{ }^{\circ} \mathrm{C}$ in five out of the eight Symbiodinium types tested (A1, B2, F1-H, F1-P, S. californium), and the effects of temperature increased with time. Type A1 showed an especially marked response to the higher temperatures, with $\mathrm{F}_{\mathrm{v}} / \mathrm{F}_{\mathrm{m}}$ being only about $18 \%$ of the control value at $34{ }^{\circ} \mathrm{C}$ after $260 \mathrm{~min}$ exposure (Mann-Whitney $\mathrm{U} ; \mathrm{Z}=-2.364$, $\mathrm{p} \leq$ 0.02; Fig. 2.1A, C). Types B2, E1-O, F1-P and S. californium also displayed a decrease in $F_{v} / F_{m}$ values relative to the control at the same time point (Mann-Whitney $U$ Test; $\mathrm{p}<0.05$ for all comparisons). In contrast, the two freshly isolated types, temperate A1-FIS and B1-FIS, did not show any response in $\mathrm{F}_{\mathrm{v}} / \mathrm{F}_{\mathrm{m}}$ to the two highest temperatures at any time point (Mann-Whitney $U, p>0.05$ in all comparisons). Unlike in the other types, $F_{v} / F_{m}$ in type $\mathrm{F} 1-\mathrm{H}$ increased slightly at $34{ }^{\circ} \mathrm{C}$ after 140 and $260 \mathrm{~min}$, and was up to $102 \%$ of the 

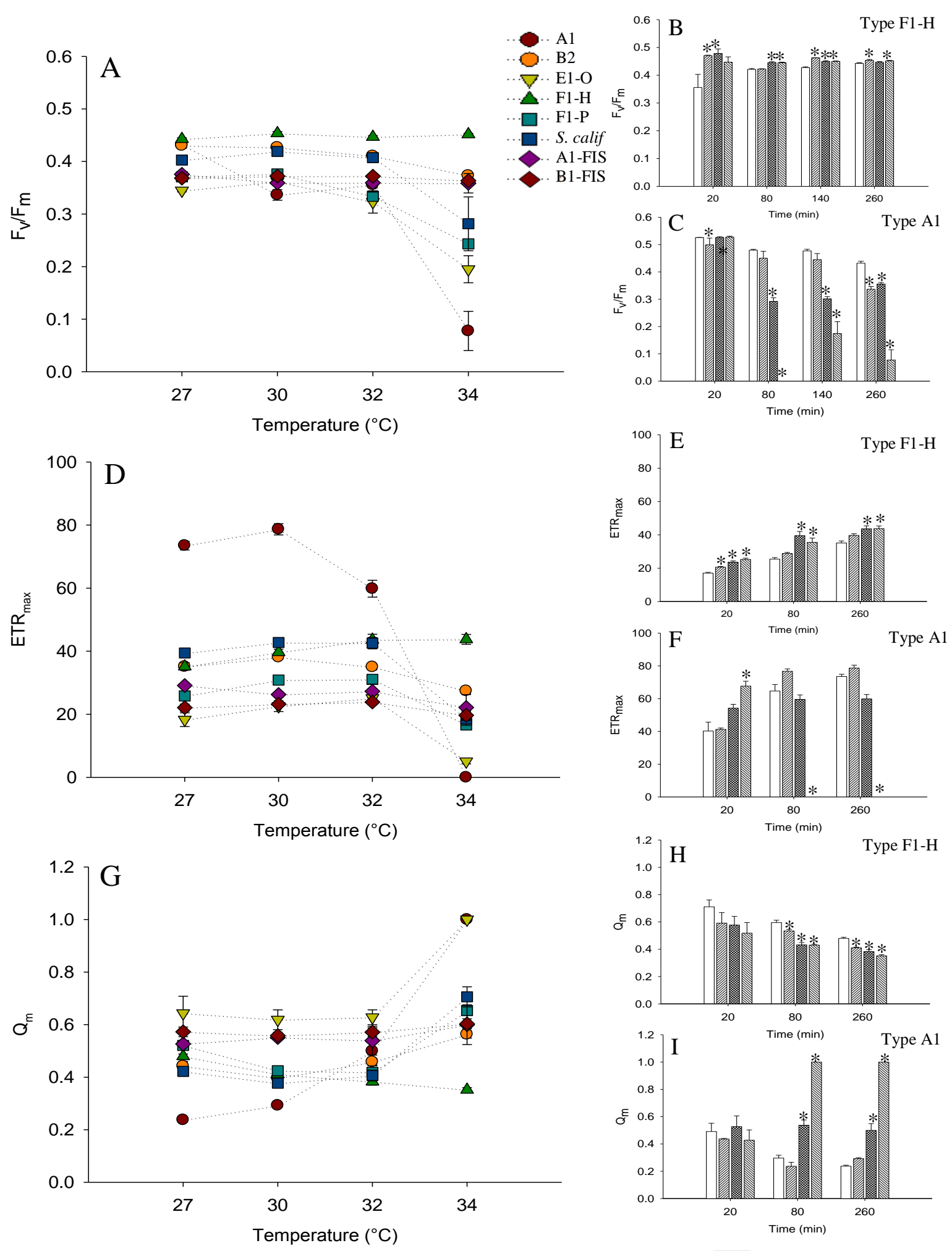

$\square 27^{\circ} \mathrm{CIIZ} 30^{\circ} \times 32^{\circ} \mathrm{C} M W \mathrm{~W} 34^{\circ} \mathrm{C}$

Figure 2.1: Effect of increasing temperature on photosynthetic capacity of Symbiodinium types (A1, B2, E1-O, F1-H, F1-P, S. californium, A1-FIS, B1-FIS); (A-C) maximum quantum yield $\left(\mathrm{F}_{\mathrm{v}} / \mathrm{F}_{\mathrm{m}}\right)$; (D-F): maximum electron transport rate $\left(\mathrm{ETR}_{\max }\right)$; $(\mathrm{G}-\mathrm{I})$ : maximum excitation pressure over photosystem II $\left(\mathrm{Q}_{\mathrm{m}}\right)$. (A, D, G) represent data after 260 min exposure; (B, C, E, F, H, I) show temporal development for each parameter for two types $(\mathrm{A} 1, \mathrm{~F} 1-\mathrm{H})$. Averages $\pm \mathrm{SE}$ shown $(\mathrm{n}=6)$; asterisks indicate significant differences $(\mathrm{p} \leq 0.05)$ between the control and treatment. 
control values at the end of the experiment (Mann-Whitney U Test; $Z=-2.250 ; p<0.025$; Fig. 2.1B).

Maximum electron transport rates $\left(\mathrm{ETR}_{\max }\right)$ varied with temperature across the different Symbiodinium types. After 260 min exposure, there was a significant change in $\mathrm{ETR}_{\max }$ at $34{ }^{\circ} \mathrm{C}$ in comparison to the control temperature (Tukey HSD, $\mathrm{p}<0.05$ for all comparisons) in all but two Symbiodinium types (A1-FIS, B1-FIS; Fig. 2.1D). As for $F_{v} / F_{m}$, ETR max $_{\text {of }}$ type A1 displayed a marked decline down to zero at $34{ }^{\circ} \mathrm{C}$ after 80 and $260 \mathrm{~min}$ (Fig. 2.1D, F). In contrast, type $\mathrm{F} 1-\mathrm{H}$ showed an increase at 32 and $34{ }^{\circ} \mathrm{C}$ relative to the control at the same time points (Tukey HSD, p < 0.05 for all comparisons; Fig. 2.1D, E).

The trends in $F_{v} / F_{m}$ and $E_{T R}$ max were also evident in the $Q_{m}$ values. Apart from $F 1-H$, A1-FIS and B1-FIS, all Symbiodinium types displayed an increase in $\mathrm{Q}_{\mathrm{m}}$ relative to the control over the exposure period (Fig. 2.1G). Type A1 showed the greatest increase from 0.240 in the control to the maximum value of 1 at $34{ }^{\circ} \mathrm{C}(422 \%)$ after $260 \mathrm{~min}$ (Tukey HSD, $\mathrm{p} \leq 0.001$; Fig. 2.1G, I). Again, the freshly isolated types A1-FIS and B1-FIS did not display significant differences between the control and $34{ }^{\circ} \mathrm{C}$ (Tukey HSD, p > 0.05 for both comparisons). By contrast, type $\mathrm{F} 1-\mathrm{H}$ decreased its $\mathrm{Q}_{\mathrm{m}}$ from 0.48 in the control to 0.35 at $34{ }^{\circ} \mathrm{C}(27 \%)$ after $260 \mathrm{~min}$ (Tukey HSD, p $\leq 0.001$; Fig. 2.1G, H).

\subsubsection{Oxidative stress experiment}

Imaging PAM chlorophyll fluorometry

$\mathrm{F}_{\mathrm{v}} / \mathrm{F}_{\mathrm{m}}$ values decreased in all Symbiodinium types following exposure to increasing $\mathrm{H}_{2} \mathrm{O}_{2}$ concentrations (rANOVA with Greenhouse-Geisser correction, $\mathrm{F}_{(60.62)}=1.58, \mathrm{p}<0.001$ ). As in the thermal stress experiment, the magnitude of the responses varied among Symbiodinium types (Fig. 2.2). $\mathrm{F}_{\mathrm{v}} / \mathrm{F}_{\mathrm{m}}$ decreased at $4 \mathrm{mM} \mathrm{H}_{2} \mathrm{O}_{2}$ relative to the control in six Symbiodinium types (B1-FIS, B2, E1-O, F1-H, F1-P, S. californium; Tukey HSD, $\mathrm{p}<0.001$ for all comparisons) after $270 \mathrm{~min}$. Type F1-H also showed a $100 \%$ decrease in $\mathrm{F}_{\mathrm{v}} / \mathrm{F}_{\mathrm{m}}$ between the control and the $2 \mathrm{mM} \mathrm{H}_{2} \mathrm{O}_{2}$ treatment after the same time period, 



Figure 2.2: Effect of increasing hydrogen peroxide $\left(\mathrm{H}_{2} \mathrm{O}_{2}\right)$ concentrations on photosynthetic capacity of Symbiodinium types (A1, B1, B2, E1, F1); (A-C) maximum quantum yield $\left(\mathrm{F}_{\mathrm{v}} / \mathrm{F}_{\mathrm{m}}\right)$; (DF): maximum rate of relative electron transport $\left(\mathrm{ETR}_{\max }\right)$; $(\mathrm{G}-\mathrm{I})$ : maximum excitation pressure over photosystem II $\left(\mathrm{Q}_{\mathrm{m}}\right)$. (A, D, G) represent data after 270 min exposure; (B, C, E, F, H, I) show temporal development for each parameter for two types $(\mathrm{A} 1, \mathrm{~F} 1-\mathrm{H})$. Averages $\pm \mathrm{SE}$ shown $(\mathrm{n}=5)$; asterisks indicate significant differences $(\mathrm{p} \leq 0.05)$ between the control and treatment. 
Chapter 2

270 min (Tukey HSD, $p<0.001$; Fig. 2.2A, C). There was no decrease in $F_{v} / F_{m}$ between the control and the $4 \mathrm{mM} \mathrm{H}_{2} \mathrm{O}_{2}$ treatments at any of the time points for the freshly isolated temperate Symbiodinium A1-FIS which showed a mean $\mathrm{F}_{\mathrm{v}} / \mathrm{F}_{\mathrm{m}}$ decrease of $17 \%$ after 270 min (Tukey HSD, p > 0.05 for all comparisons; Fig. 2.2A, B).

The $F_{v} / F_{m}$ results are consistent with changes in maximum ETR which decreased over time in all Symbiodinium types (rANOVA with Greenhouse-Geisser correction, $\mathrm{F}_{(13.34)}=4.20$, $\mathrm{p}<0.001$; Fig. 2.2D). Types B1 and F1-H again showed the greatest response with the latter displaying an $\mathrm{ETR}_{\max }$ value of zero in the $4 \mathrm{mM} \mathrm{H}_{2} \mathrm{O}_{2}$ treatment after only $30 \mathrm{~min}$ exposure time (Tukey HSD, p < 0.001; Fig. 2.2D, F). None of the other Symbiodinium types used showed significant changes between treatments at the same time point (Tukey

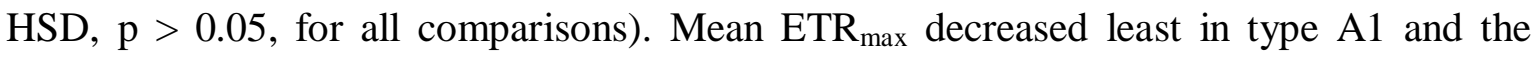
freshly isolated temperate type A1-FIS which showed a decline of only $11 \%$ between the control and $4 \mathrm{mM} \mathrm{H}_{2} \mathrm{O}_{2}$ treatments (Fig. 2.2D, E).

$\mathrm{Q}_{\mathrm{m}}$ values increased over time (Greenhouse-Geisser, $\mathrm{F}_{(14.582)}=2.139, \mathrm{p} \leq 0.010$ ). Both $\mathrm{B} 1$ and $\mathrm{F} 1-\mathrm{H}$ reached the maximum $\mathrm{Q}_{\mathrm{m}}$ value of 1 after 30 min exposure to $4 \mathrm{mM} \mathrm{H}_{2} \mathrm{O}_{2}$ with F1-H increasing from a value of 0.47 in the control (113\%; Tukey HSD, $p \leq 0.001$; Fig. 2.2G, I). No significant increase in $\mathrm{Q}_{\mathrm{m}}$ was observed over this same period in types $\mathrm{A} 1$ and E1-O as well as in the freshly isolated temperate type A1-FIS. The latter did not display any increase in $\mathrm{Q}_{\mathrm{m}}$ in response to any of the treatments at any time point (Tukey HSD, p > 0.050; Fig. 2.2G, H).

$\mathrm{O}_{2}$ fluxes

Rates of maximum photosynthesis $(\mathrm{P})$ and dark respiration $(\mathrm{R})$ were measured with an $\mathrm{O}_{2}$ electrode. These data corroborated the chlorophyll fluorescence measurements, with mean rates of photosynthesis $(\mathrm{P})$ and respiration $(\mathrm{R})$ declining in all Symbiodinium types with the addition of $\mathrm{H}_{2} \mathrm{O}_{2}$ (Two-way ANOVA, $\mathrm{F}_{(4)}=32.58, \mathrm{p}<0.001$; Fig. 2.3). Maximum photosynthesis decreased in half of the Symbiodinium types (B2, E1-O, F1-H, S. californium) at the highest $\mathrm{H}_{2} \mathrm{O}_{2}$ concentration, $4 \mathrm{mM}$ (Tukey HSD, $\mathrm{p} \leq 0.04$ for all comparisons). While type $\mathrm{F} 1-\mathrm{H}$ displayed a photosynthetic rate only $3.8 \%$ of the control 

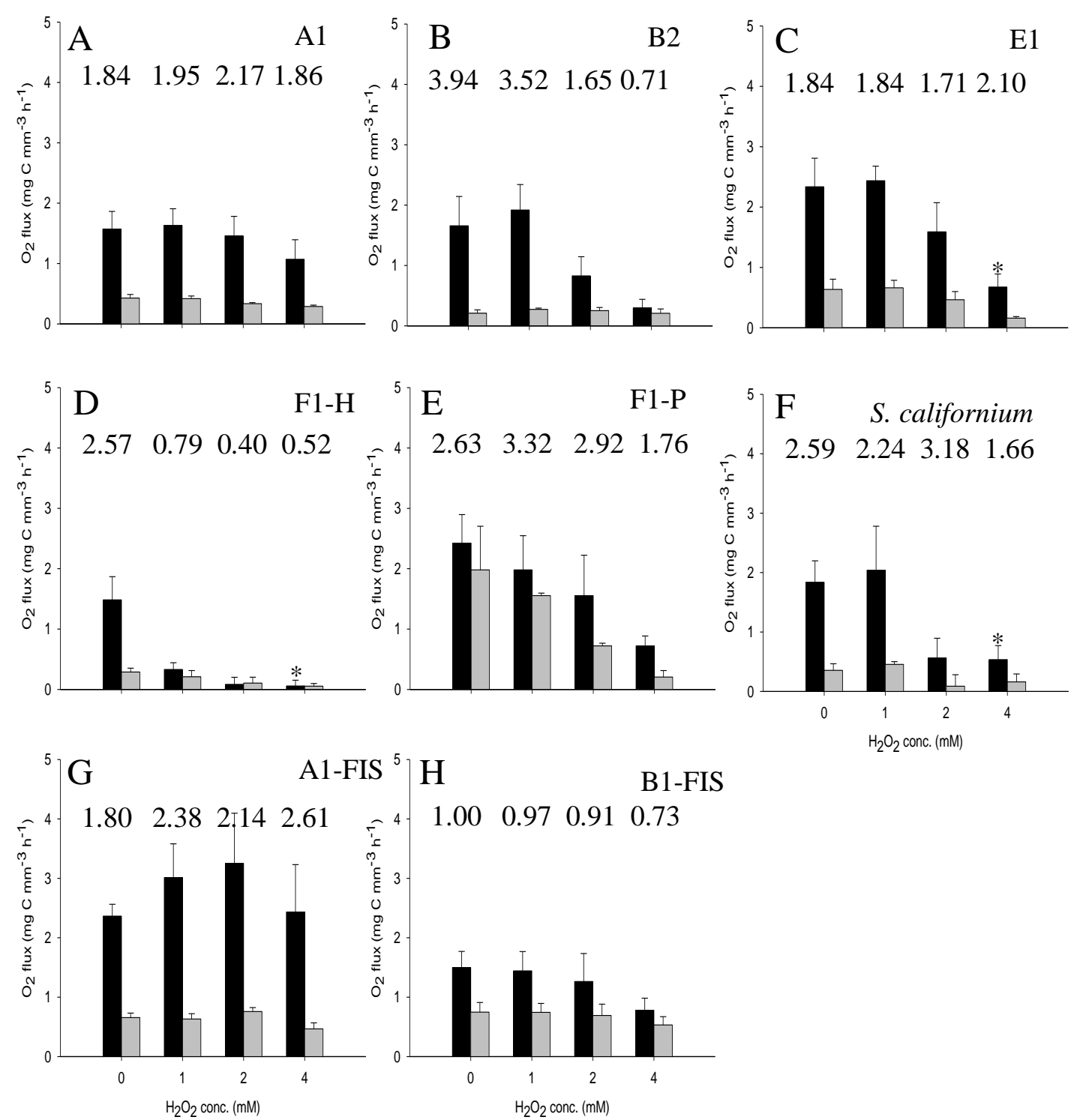

Figure 2.3: Effect of increasing hydrogen peroxide $\left(\mathrm{H}_{2} \mathrm{O}_{2}\right)$ on oxygen fluxes $\left(\mathrm{mg} \mathrm{C} \mathrm{mm}^{-3} \mathrm{~h}^{-1}\right)$ of Symbiodinium types (A1, B1, B2, E1, F1); (A-G) photosynthesis (P, dark bar) and respiration (R, light bar) per cell volume with $P: R$ ratio given above bars. Averages \pm SE shown $(n=5)$; asterisks indicate significant differences $(\mathrm{p} \leq 0.05)$ between the control and treatments.

value at $4 \mathrm{mM} \mathrm{H} \mathrm{H}_{2} \mathrm{O}_{2}$ (Tukey $\mathrm{HSD}, \mathrm{p}<0.05$; Fig. 2.3D), the freshly isolated temperate Symbiodinium type A1-FIS maintained its photosynthetic rate (Tukey HSD, p > 0.05; Fig. 2.3F). This physiological response was reflected in the P:R value (an indicator of autotrophic potential), which declined in most of the Symbiodinium types (B1-FIS, B2, F1-H, F1-P, S. californium) in response to the $4 \mathrm{mM} \mathrm{H}_{2} \mathrm{O}_{2}$ treatment. In three of these types (B1-FIS, B2, F1-H), the P:R value became lower than 1, with a minimum value of 
Chapter 2

0.5 in type F1-H (Tukey HSD, p < 0.05 for P; Fig. 2.3F). The P:R value also decreased from 2.6 to 1.8 and 1.7 between the control and the $4 \mathrm{mM} \mathrm{H}_{2} \mathrm{O}_{2}$ treatments in type F1-P and the temperate $S$. californium (Fig. 2.3E, F), respectively. In contrast, the P:R value of some Symbiodinium types remained the same between the control and the highest $\mathrm{H}_{2} \mathrm{O}_{2}$ treatment, as seen in type A1 (1.84 to 1.86; Tukey HSD, p < 0.015 for P and R; Fig. 2.3A) or it even increased as seen in type A1-FIS (1.8 to 2.6; Tukey HSD, p > 0.05 for P and R; Fig. 2.3F).

\subsection{Discussion}

This study showed that the various Symbiodinium types responded to elevated thermal and oxidative stress, as would be expected. Generally, a decrease in photosynthetic capacity was observed with increasing stress over time. However, the various Symbiodinium types showed marked differences in their response and therefore their susceptibilities to these stressors. Interestingly, these responses differed not only among types but also within single types, in terms of their relative susceptibilities to the two stressors (Table 2.2).

Both thermal and oxidative stress seemed to affect the algal cells either in direct or indirect damage to the function of the photosystems. This was illustrated by the reductions in $F_{v} / F_{m}$ and $\mathrm{ETR}_{\max }$ and, in the case of oxidative stress, decreasing rates of photosynthetic $\mathrm{O}_{2}$ production and respiratory $\mathrm{O}_{2}$ consumption with increasing hydrogen peroxide levels, as previously shown by Jones et al. (2000) and Marutani et al. (2012). Concurrent with these effects, $\mathrm{Q}_{\mathrm{m}}$, which I used as a proxy for non-photochemical quenching (NPQ), increased with elevated stress, consistent with an increase in excitation pressure over PSII (Abrego et al. 2008). However, while thermal and oxidative stress both impacted cellular photophysiology in similar ways, there were clear differences between the different Symbiodinium types, with some types being more resistant to the stress than others. The mechanisms that explain these differential stress tolerances are unclear. 
Table 2.2: Simplified summary of results with different Symbiodinium types (in culture, freshly isolated) under thermal stress and hydrogen peroxide $\left(\mathrm{H}_{2} \mathrm{O}_{2}\right)$ induced oxidative stress. $\uparrow$ and $\downarrow$ arrows represent increase and decrease of measured parameters $\left(\mathrm{F}_{\mathrm{v}} / \mathrm{F}_{\mathrm{m}}, \mathrm{ETR}_{\max }, \mathrm{Q}_{\mathrm{m}}, \mathrm{P} / \mathrm{R}\right)$ respectively, while - represents no change.

\begin{tabular}{|l|l|l|l|l|l|l|l|}
\hline & \multicolumn{3}{|c|}{ Thermal stress } & \multicolumn{5}{c|}{ Oxidative stress $\left(\mathbf{H}_{2} \mathbf{O}_{2}\right)$} \\
\hline Type & $\mathbf{F}_{\mathbf{v}} / \mathbf{F}_{\mathbf{m}}$ & $\mathbf{E T R}_{\max }$ & $\mathbf{Q}_{\mathbf{m}}$ & $\mathbf{F}_{\mathbf{v}} / \mathbf{F}_{\mathbf{m}}$ & $\mathbf{E T R}_{\max }$ & $\mathbf{Q}_{\mathbf{m}}$ & $\mathbf{P} / \mathbf{R}$ \\
\hline A1 & $\downarrow \downarrow \downarrow$ & $\downarrow \downarrow \downarrow$ & $\uparrow \uparrow \uparrow$ & - & $\downarrow$ & $\uparrow$ & - \\
\hline B2 & $\downarrow$ & $\downarrow$ & $\uparrow$ & $\downarrow \downarrow \downarrow$ & $\downarrow \downarrow \downarrow$ & $\uparrow \uparrow \uparrow$ & $\downarrow \downarrow \downarrow$ \\
\hline E1-O & $\downarrow \downarrow$ & $\downarrow \downarrow \downarrow$ & $\uparrow \uparrow \uparrow$ & $\downarrow \downarrow$ & $\downarrow \downarrow$ & $\uparrow \uparrow$ & $\uparrow$ \\
\hline F1-H & $\uparrow$ & $\uparrow$ & $\downarrow$ & $\downarrow \downarrow \downarrow$ & $\downarrow \downarrow \downarrow$ & $\uparrow \uparrow \uparrow$ & $\downarrow \downarrow \downarrow$ \\
\hline F1-P & $\downarrow \downarrow$ & $\downarrow \downarrow$ & $\uparrow \uparrow$ & $\downarrow \downarrow$ & $\downarrow \downarrow$ & $\uparrow \uparrow$ & $\downarrow$ \\
\hline S. californium & $\downarrow \downarrow$ & $\downarrow \downarrow$ & $\uparrow \uparrow$ & $\downarrow \downarrow$ & $\downarrow \downarrow$ & $\uparrow \uparrow$ & $\downarrow$ \\
\hline & & & & & & & \\
\hline A1-FIS & - & - & - & - & - & - & $\uparrow$ \\
\hline B1-FIS & - & - & - & $\downarrow \downarrow$ & $\downarrow \downarrow$ & $\uparrow$ & $\downarrow \downarrow$ \\
\hline
\end{tabular}

Several key protective mechanisms have been identified as being potentially important in controlling thermal tolerance in Symbiodinium; these are NPQ, D1 repair, heat shock proteins and thylakoid composition. NPQ is a protective mechanism, in which the singletstate excitation of a chlorophyll $a$ molecule is returned to ground state and excess photosynthetic energy is dissipated as heat (Müller et al. 2001, Hill et al. 2005). It may therefore follow that symbionts that are better at dissipating excess photochemical energy through NPQ are less susceptible to thermal bleaching (Warner et al 1999, Stambler and Dubinsky 2004). NPQ also leads to a decrease in photosynthetic efficiency and production (Smith et al. 2005), so the responses of the more sensitive Symbiodinium types here are consistent with a photo-protective role for NPQ. However, the relative thermal tolerance of Symbiodinium type F1-H cannot be explained by this mechanism alone, as it showed no decline in its photosynthetic performance with increased temperature and excitation pressure over PSII $\left(\mathrm{Q}_{\mathrm{m}}\right)$ did not increase. It may well be that this Symbiodinium type utilizes additional mechanisms to protect itself. For example, damage to PSII and especially the D1 protein might be compensated for by a faster rate of repair (Takahashi et al. 2008); this could prevent chronic photo-inhibition and higher rates of repair could indicate increased thermal tolerance (Ragni et al. 2010, Hennige et al. 2011). Also, dinoflagellates can synthesize heat shock proteins (Hsps) when they are briefly exposed to high temperatures as well as other stressful situations, and their generation correlates with the acquisition of enhanced thermotolerance (Black et al. 1995, Leggat et al. 2007). During 
stressful conditions, Hsps chaperone proteins thereby help to maintain protein structure and function (Fang et al. 1997, Leggat et al. 2011). Furthermore, the fatty acid composition of the dinoflagellate's thylakoid membranes may confer thermotolerance, with higher thermotolerance being linked to a lower content of the major polyunsaturated fatty acid, $\Delta 6,9,12,15$-cis-octadecatraenoic acid relative to $\Delta 9$-cis-octadecatetraenoic acid (Tchernov et al. 2004). The extent to which these various mechanisms explain the diverse trends seen in the current study are unknown and require further exploration.

Most pertinent to the current study is the role of antioxidants in controlling ROS, and hence damage to cellular structure and function. The production of ROS is a consequence of high temperature as well as other biotic and abiotic stressors (Alscher et al. 1997), and it is conceivable that the differential responses to thermal stress seen among Symbiodinium types are linked to differing capacities to deal with ROS and oxidative stress. Possible antioxidants include carotenoids, whose pool is decreased through xanthophyll cycling (Dove et al. 2006), itself a central mechanism of NPQ (Lesser 2006). Carotenoids are lipid soluble antioxidants that not only function as accessory pigments in light harvesting, but also quench ROS and prevent lipid peroxidation (Fridovich 1998). In addition, a number of other non-enzymatic, as well as enzymatic antioxidants may be generated in the dinoflagellate chloroplast and mitochondria (Lesser 2006). Key enzymes include SOD, which reduces superoxide to $\mathrm{H}_{2} \mathrm{O}_{2}$; this is subsequently reduced to water and oxygen by CAT and APX (Halliwell and Gutteridge 1989, Lesser 2006).

Symbiodinium type F1-H performed well at high temperature but was the least tolerant type to the addition of $\mathrm{H}_{2} \mathrm{O}_{2}$. One possible reason for this is that, due to its thermally robust nature (perhaps resulting from one or more of the mechanisms described above) this Symbiodinium type is not predisposed to dealing with high levels of oxidative stress. In contrast, type A1 was especially sensitive to high temperature but was quite tolerant of $\mathrm{H}_{2} \mathrm{O}_{2}$. In this case, the dinoflagellate may have adapted to a more frequent production of $\mathrm{H}_{2} \mathrm{O}_{2}$ in response to thermal change (Suggett et al. 2008) by acquiring a more efficient antioxidant defence system. The same might also apply to $S$. californium (E1). 
Many of the Symbiodinium types used in this study experience variable temperatures in their natural environments. For instance, sea water temperatures in the Gulf of Aqaba (A1) range seasonally from 20 to $27^{\circ} \mathrm{C}$ (Manasrah et al. 2006) and a similar range is observed in Okinawa (E1) where in extreme cases, temperatures vary between 21 and $30{ }^{\circ} \mathrm{C}$ (Surfforecast.com). These types were in general more resilient to thermal and oxidative stress than types from less variable environments: for example, type F1 from Hawaii was very susceptible to just a small increase in $\mathrm{H}_{2} \mathrm{O}_{2}$ concentration, while type E1 from Okinawa was a lot more resilient. It is notable that the two Symbiodinium types from temperate environments (type A1-FIS from Wellington, New Zealand and S. californium E1) from southern California, USA) were amongst the most resistant to both thermal and oxidative stress. In their natural habitats, A1-FIS and $S$. californium experience much lower minimum and average temperature than the other types, with averages ranging from 8-19.6 ${ }^{\circ} \mathrm{C}$ (Greater Wellington Regional Council) and 13-20 ${ }^{\circ} \mathrm{C}$ (NOAA), respectively. In addition, these two types come from high latitudes, and experience intertidal environments with variable irradiance and temperature. These highly variable conditions with more extreme temperature and irradiance fluctuations than in tropical environments (MullerParker and Davy 2001) could be the cause for the heightened tolerance to various stressors as observed in this and other studies (Kübler and Davison 1993, Bingham et al. 2011). That different Symbiodinium types, even within the same clade, exhibit different responses to stress is well known (Warner et al. 1999, Tchernov et al. 2004, Sampayo et al. 2007), and it is not surprising that corals and their symbionts growing in more high latitude environments should cope better with relatively variable conditions (Wicks et al. 2010). The basis for this environmental tolerance remains elusive, but the results suggest a link between environmental conditions and perhaps the relative importance of the different mechanisms used to defend against thermal and oxidative stress. Symbiodinium types from a far wider range of habitats are needed to test this further, though very few types of high latitude Symbiodinium are currently in culture around the world and this has limited the potential for latitudinal comparisons of Symbiodinium physiology.

This study clearly demonstrated a diversity of responses to thermal and oxidative stress within the genus Symbiodinium. These results point out the difficulty of predicting Symbiodinium stress responses, eg to temperature increases as a consequence of climate 
Chapter 2

change. Not only the Symbiodinium type needs to be considered but the algal cell's geographic origin and thermal history. Furthermore, the coral host's influence on bleaching tolerance, eg through its own generation of antioxidants, should be taken into account when looking at Symbiodinium cells in hospite in future research. It was also shown that sensitivity to oxidative stress did not necessarily result in reduced thermal tolerance, as had been hypothesised. The interrelationship between thermal and oxidative stress resistance therefore remains unclear, though further elucidation of the physiological mechanisms used to tolerate heat stress, as well as non-enzymatic and enzymatic antioxidant pathways, will help us to unravel the complexities of this important topic. 


\section{Chapter 2}




\section{Chapter 3: ROS generation in response to thermal and oxidative stress in different Symbiodinium types}

\subsection{Introduction}

Oxygen is produced by the light-driven splitting of water during photosynthesis in cyanobacteria, algae and higher plants. In its elemental form, oxygen is used in cellular respiration in all complex organisms (Lesser 2012). However, while these aerobic processes are efficient, the presence of oxygen at the cellular level creates a constant oxidative threat (Veljovic -Jovanovic 1998). Electrons, stemming from electron transport between cell organelles, react with molecular oxygen in plant cells and initiate the generation of reactive oxygen species (ROS; Weis 2008). Increases of ROS production can be caused by biotic stresses and abiotic ones, such as high light or temperature, where the first site of action is the interior of the cell, within the chloroplast (Alscher et al. 1997). While these processes can occur in all plant cells, in marine systems they are particularly important in the context of coral reefs and the functional biology of the symbiotic dinoflagellates (genus Symbiodinium) of corals.

Symbiodinium spp. live as endosymbionts in various hosts, including many cnidarians (e.g. corals, sea anemones, jellyfish). By contributing significantly to the productivity and survival of their host, these dinoflagellate symbionts have been key to the success of scleractinian (stony) corals as reef builders. However, this relationship is under threat due to climate change and higher-than-normal seawater temperatures, which work in tandem with high irradiance to stress corals (Hoegh-Guldberg et al. 2007). Heat and light can cause damage in the light and dark reactions of the symbiotic alga cell's photosynthetic apparatus (also see section 1.3), leading to the generation of ROS such as superoxide $\left(\mathrm{O}_{2}^{-}\right)$, singlet oxygen $\left({ }^{1} \mathrm{O}_{2}\right)$, hydrogen peroxide $\left(\mathrm{H}_{2} \mathrm{O}_{2}\right)$ and the highly reactive hydroxyl radical $\left(\mathrm{OH}^{\circ}\right.$; Lesser 2006). ROS can damage cell organelles of the algal symbiont and the coral host, and cause decreased growth, and increased susceptibility to disease in the host (Lesser 2012). Importantly, ROS can also lead to the expulsion of the symbiotic dinoflagellates from the host's cells in so-called coral bleaching events, therefore disrupting the symbiosis and often causing the death of the animal host (Tchernov et al. 
2004, Smith et al. 2005). It is therefore imperative that photosystem damage is counteracted, for instance with the biosynthesis of antioxidants (Alscher et al. 1997). In algae, daily as well as seasonal cycling of antioxidant production has been observed, with peaks during maximum light intensities at midday and during summer (Butow et al. 1997a, b, Lesser 2006), and at least one study shows transcriptional control of these cycles (Okamoto et al. 2001). Moreover, in plants, it appears that antioxidant levels are responsible for significant developmental differences in sensitivities to oxidative stress, as studies on peas have demonstrated (Donahue et al. 1997).

To measure ROS production in cells, indirect indices such as DNA and protein damage have been traditionally used (Halliwell and Gutteridge 1989). However, a more recent and precise approach is the combination of fluorescent probes and laser-scanning confocal microscopy, which can identify relative quantities and sites of photo-oxidative stress and ROS generation (Fryer et al. 2002, Wrona and Wardman 2006, Wang et al. 2011). Especially useful are the positive fluorogenic probes that are non-fluorescent (or weakly fluorescent), but yield fluorescent products upon reaction with ROS (Gomes et al. 2005, Bartosz 2006, Soh 2006, Driever et al. 2009). Antioxidant capacity can also be measured via fluorescent probes that detect ROS. Superoxide dismutase (SOD) reduces $\mathrm{O}_{2}{ }^{-}$to $\mathrm{H}_{2} \mathrm{O}_{2}$, which can be reduced by ascorbate peroxidase (APX) and catalase (CAT), while ${ }^{1} \mathrm{O}_{2}$ is reduced by $\beta$-carotene and $\alpha$-tocopherol, both to water and oxygen (Venn et al. 2008). These antioxidants react with pro-oxidants to produce water and oxygen (Weis 2008). Meanwhile, the probes react with any un-reacted pro-oxidants and produce fluorescent oxidation products (Wolfe and Liu 2007). Therefore, as antioxidant activity increases, the signal from the fluorescent product decreases. At the same time, laser-scanning confocal microscopy enables the generation of two- or three-dimensional images of the cells being studied. Fluorescent probes inside the cell make ROS and antioxidants visible, and provide information about their relative quantities and site(s) of activity in vivo (Flors et al. 2006, Ashtamker et al. 2007).

In this study, I used fluorescent probes to tag different ROS generated during either thermal or oxidative stress inside cultured Symbiodinium cells. Different Symbiodinium sub-clades ('types') from high and low latitudes were chosen. Changes in ROS abundance 
Chapter 3

were measured via flow cytometry, with concomitant confocal microscopy for visualization. I predicted a diverse range of thermal- and oxidative-stress responses consistent with the geographical origins of these Symbiodinium types, and that the responses to these two stressors would be similar in terms of ROS production.

\subsection{Material and Methods}

\subsubsection{Symbiodinium types and cultivation}

Five different types of cultured Symbiodinium (A1, B2, E1-O, F1, S. californium) were used for the experiments (Table 3.1). For culture identification in this chapter, see Chapter 2. These were grown in silica-free f/2 culture medium (NCMA, East Boothbay, Maine, USA) at $26 \pm 1{ }^{\circ} \mathrm{C}$ on a $12 \mathrm{~h}$ light: $12 \mathrm{~h}$ dark cycle $\left(55 \pm 5 \mu\right.$ mol photons $\mathrm{m}^{-2} \mathrm{~s}^{-1}, 36 \mathrm{~W}$ white fluorescent tubes, Philips TL-D) for more than a year beforehand. Cultures were subcultured every 6-8 weeks. All experiments were conducted two weeks after sub-culturing when they were still in the log growth phase.

\subsubsection{Genetic identification of Symbiodinium types}

Protocols by Stat et al. (2009) and Logan et al. (2010) for DNA extraction of the different types was used, except that the duration of the DNA extraction was adjusted to five days. DNA was added to MyTaq ${ }^{\mathrm{TM}}$ mastermix (Bioline, London, UK) with Symbiodinium specific ITS2 primers (10 mM; forward primer: GTG AAT TGC AGA ACT CCG TG; reverse primer: CCT CCG CTT ACT TAT ATG CTT) and double distilled (dd) $\mathrm{H}_{2} \mathrm{O}$ to give a final volume of $25 \mu \mathrm{L}$. Subsequently, a PCR was run on samples $\left(3\right.$ min at $95{ }^{\circ} \mathrm{C}$, 28 cycles of $15 \mathrm{~s}$ at $95^{\circ} \mathrm{C}, 15 \mathrm{~s}$ at $57{ }^{\circ} \mathrm{C}, 10 \mathrm{~s}$ at $\left.72{ }^{\circ} \mathrm{C}\right)$. Successful PCR was confirmed via $1.5 \%$ agarose gel (Sigma Aldrich New Zealand Ltd., $120 \mathrm{~V}, 45 \mathrm{~min}$ ) which was stained with ethidium bromide (final concentration $0.5 \mu \mathrm{L} \mathrm{mL}^{-1}$ ). The final PCR product was purified with Affymetrix USB ExoSAP-IT (In Vitro Technologies, Victoria, Australia) according to manufacturer's instructions and sent away for sequencing to Macrogen Inc. (Seoul, Korea). 
Chapter 3

Table 3.1: Symbiodinium cultures with corresponding host species, geographic origin and subclade.

\begin{tabular}{|l|l|l|l|}
\hline Culture I.D. & Origin host species & Geographic origin* & Sub-clade \\
\hline CCMP2467 & Stylophora pistillata & Gulf of Aqaba & A1 \\
\hline CCMP2459 & Oculina diffusa & Bermuda & B2 \\
\hline A001 & Acropora sp. & Okinawa & E1 \\
\hline Mv & Montipora verrucosa & Hawaii & F1 \\
\hline S. californium & Anthopleura elegantissima & Southern California & E1 \\
\hline
\end{tabular}

* for more detailed information, see Table 2.1

\subsubsection{Experimental set-up}

Symbiodinium cultures were centrifuged $\left(1800 \times g, 5 \mathrm{~min}, 25^{\circ} \mathrm{C}\right)$ and the $\mathrm{f} / 2$ medium was replaced with filtered seawater $(1 \mu \mathrm{m}$-FSW) to remove any pre-existing ROS. The final concentration was adjusted to $1 \times 10^{6}$ cells $\mathrm{mL}^{-1}$, as determined by haemocytometer counts ( $\times 100$ magnification, $\mathrm{n}=6$ counts per Symbiodinium cell suspension). Cell suspensions were then evenly distributed into $15 \mathrm{~mL}$ centrifuge tubes $(2 \mathrm{~mL}$ per tube) for different treatments.

\subsubsection{Thermal stress experiment}

For thermal stress experiments, two water baths at different temperatures $\left(26 \pm 1{ }^{\circ} \mathrm{C}\right.$ and $35 \pm 1{ }^{\circ} \mathrm{C}$ ) were used. Each water bath contained tubes for the control and two treatments with different fluorescent probes ( $\mathrm{n}=3$ per treatment). The treatment temperature of $35^{\circ} \mathrm{C}$ was used, as it is considered stressful under natural conditions (Fitt et al. 2009, Rosic et al. 2011) and has proven so in earlier experiments (see Chapter 2 of this thesis).

Control tubes contained Symbiodinium cells in FSW only. For the first treatment, $100 \mu \mathrm{M}$ 2',7'-dichlorodihydrofluorescein diacetate $\left(\mathrm{H}_{2}\right.$ DCF-DA) in dimethyl sulfoxide (DMSO) was added to cells. A combination of $1 \mathrm{mM}$ hydroethidine and $1 \mathrm{mM}$ singlet oxygen sensor green (SOG) was added for the second treatment (1 $\mu \mathrm{L}$ probe $1 \mathrm{~mL}^{-1}$ cell suspension). 
Chapter 3

$\mathrm{H}_{2}$ DCF-DA is a general indicator for ROS, emitting green fluorescence once the acetate group is removed, while hydroethidine is oxidised by superoxide to 2-hydroxyethidium emitting a bright red fluorescence. Singlet Oxygen Sensor Green reagent is highly selective for singlet oxygen and emits green fluorescence in its presence. The advantages of these three probes include high sensitivity and a linear response to a wide range of ROS concentrations (all chemicals: Invitrogen, Life Technologies, Grand Island, NY, USA). Before treatments were placed in water baths, cells were incubated in the dark for one hour at $26{ }^{\circ} \mathrm{C}$ and a first baseline measurement with the flow cytometer (FACScan, BD Biosciences, USA; 10,000- 50,000 cells measured, flow rate $60 \mu \mathrm{L} \mathrm{min}{ }^{-1}$, threshold 52 Forward scatter, FSC) was taken. After the first measurement, one set of tubes of each treatment (control; $+\mathrm{H}_{2} \mathrm{DCF}-\mathrm{DA}$; + dihydroethidine and $\mathrm{SOG}$ ) was placed in a water bath, either at $26 \pm 1{ }^{\circ} \mathrm{C}$ or $35 \pm 1{ }^{\circ} \mathrm{C}$. Cell suspensions were kept in darkness throughout the exposure period to prevent degradation of the fluorescent probes, and sub-samples (minimum $300 \mu \mathrm{L}$ ) were taken after 1, 2 and $4 \mathrm{~h}$ for measurements in the flow cytometer. For data analysis, control values were subtracted from the values for treatment samples at each temperature, to correct for any autofluorescence.

\subsubsection{Oxidative stress experiment}

To test the effects of oxidative stress induced by hydrogen peroxide $\left(\mathrm{H}_{2} \mathrm{O}_{2}\right)$ on Symbiodinium cells, a similar experimental design was used as in the thermal stress experiment. This time, all tubes were kept at room temperature $\left(25 \pm 1{ }^{\circ} \mathrm{C}\right)$ in the dark and included a control and four treatments ( $n=3$ per treatment).

Control tubes again contained cells but no probe. For the first and second experimental treatment, $\mathrm{H}_{2}$ DCF-DA, and a combination of dihydroethidine and SOG was added to the algal cells, respectively. For the third and fourth treatment, tubes with cells were prepared in the same way as for the first two treatments. However, before the first flow cytometer measurement, $\mathrm{H}_{2} \mathrm{O}_{2}$ at a final concentration of $8 \mathrm{mM}$ was added to Symbiodinium cells. A final concentration of $8 \mathrm{mM} \mathrm{H} \mathrm{H}_{2} \mathrm{O}_{2}$ was used for the oxidative stress experiment as this concentration had been found to invoke a response in all Symbiodinium types (Chapter 2). Fluorescence values for cells only were subtracted from each treatment before data analysis. Measurements were taken at 1,2 and 4 hours. 
Chapter 3

\subsubsection{Confocal microscopy}

Confocal microscopy was used to corroborate the data obtained with the flow cytometer by visualising the uptake of fluorescent probes inside the Symbiodinium cells (Fig. 3.1). Samples were prepared as described above. Confocal microscope images (Olympus IX81 FV1000, Olympus Europa Holding GmbH, Germany) were taken before and after the addition of $\mathrm{H}_{2} \mathrm{O}_{2}$ with the different fluorescent probes over the course of the $4 \mathrm{~h}$ exposure period.

\subsubsection{Statistical analysis}

Normality was tested using Shapiro Wilk's test. For all data sets from the thermal stress experiment, the non-parametric repeated measure Friedman Test was performed. Subsequently, post hoc Wilcoxon Rank Tests $(\mathrm{p} \leq 0.05)$ and Mann-Whitney U Tests with Bonferroni correction $(\mathrm{p} \leq 0.017)$ were conducted to look at differences between means. Normally distributed data from the oxidative stress experiment were transformed and subjected to a repeated measures ANOVA (Symbiodinium type $\mathrm{x}_{2} \mathrm{H}_{2}$ concentration). Separate one-way ANOVAs were performed to look at the treatment effect (one-way ANOVA, $\mathrm{p} \leq 0.05$ ) and post hoc tests on differences between means within Symbiodinium types were conducted (LSD, $\mathrm{p} \leq 0.05)$.

For all data analyses, PASW Statistics 18 was used (IBM SPSS, IBM Corp., Armonk, NY, USA). 

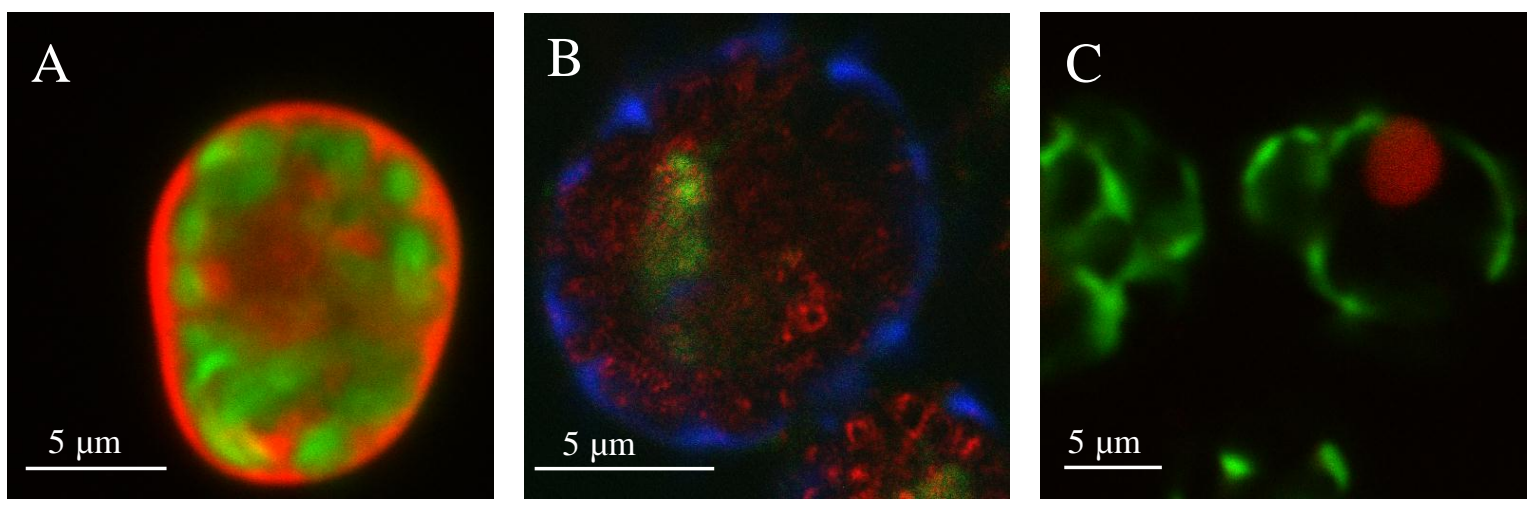

Figure 3.1: Symbiodinium cells with different fluorescent probes marking reactive oxygen species (ROS). (A) green: 2',7'-dichlorodihydrofluorescein diacetate $\left(\mathrm{H}_{2} \mathrm{DCF}-\mathrm{DA}\right)$ reacting with ROS in general, red: chlorophyll autofluorescence; (B) blue: unreacted dihydroethidine demonstrating no presence of superoxide, green: singlet oxygen sensor green (SOG) reacting with singlet oxygen, red: chlorophyll autofluorescence; (C) red: reacted dihydroethidine showing presence of superoxide, green: singlet oxygen sensor green (SOG; scale bars: $5 \mu \mathrm{m}$ ).

\subsection{Results}

\subsubsection{Thermal stress experiment}

The effect of increased temperature on the generation of ROS in five different Symbiodinium types was tested using specific fluorescent probes. Changes in fluorescence (i.e. ROS concentration) were detected via flow cytometry (Fig. 3.2).

When measuring overall ROS using the probe $\mathrm{H}_{2}$ DCF-DA, there was an overall significant effect of time (Friedman Test, $\chi^{2}=26.867, \mathrm{df}=2, \mathrm{p}<0.001$ ). In the E1 Symbiodinium type (E1-O and S. californium) no differences could be detected between the control and the temperature treatments at 1- 4 h (Fig. 3.2Ci, Ei). In Symbiodinium types A1 and B2, cells at $35{ }^{\circ} \mathrm{C}$ displayed higher fluorescence levels than at $26{ }^{\circ} \mathrm{C}$ after 4 hours (Mann-Whitney $\mathrm{U}$ Test, $\mathrm{Z}=-1.964, \mathrm{p}<0.05$ for both comparisons; Fig. 3.2Ai, Bi). Additionally, fluorescence at $35{ }^{\circ} \mathrm{C}$ was also higher than at $26{ }^{\circ} \mathrm{C}$ after two hours of exposure in type A1 (MannWhitney U Test, $\mathrm{Z}=-1.964, \mathrm{p}<0.05)$. In Symbiodinium type F1, fluorescence after $4 \mathrm{~h}$ exposure to $35{ }^{\circ} \mathrm{C}$ was higher than at $26{ }^{\circ} \mathrm{C}$ at the same time point (Mann-Whitney U Test, $\mathrm{Z}=-1.993, \mathrm{p}<0.05$; Fig. 3.2Di). 
Chapter 3
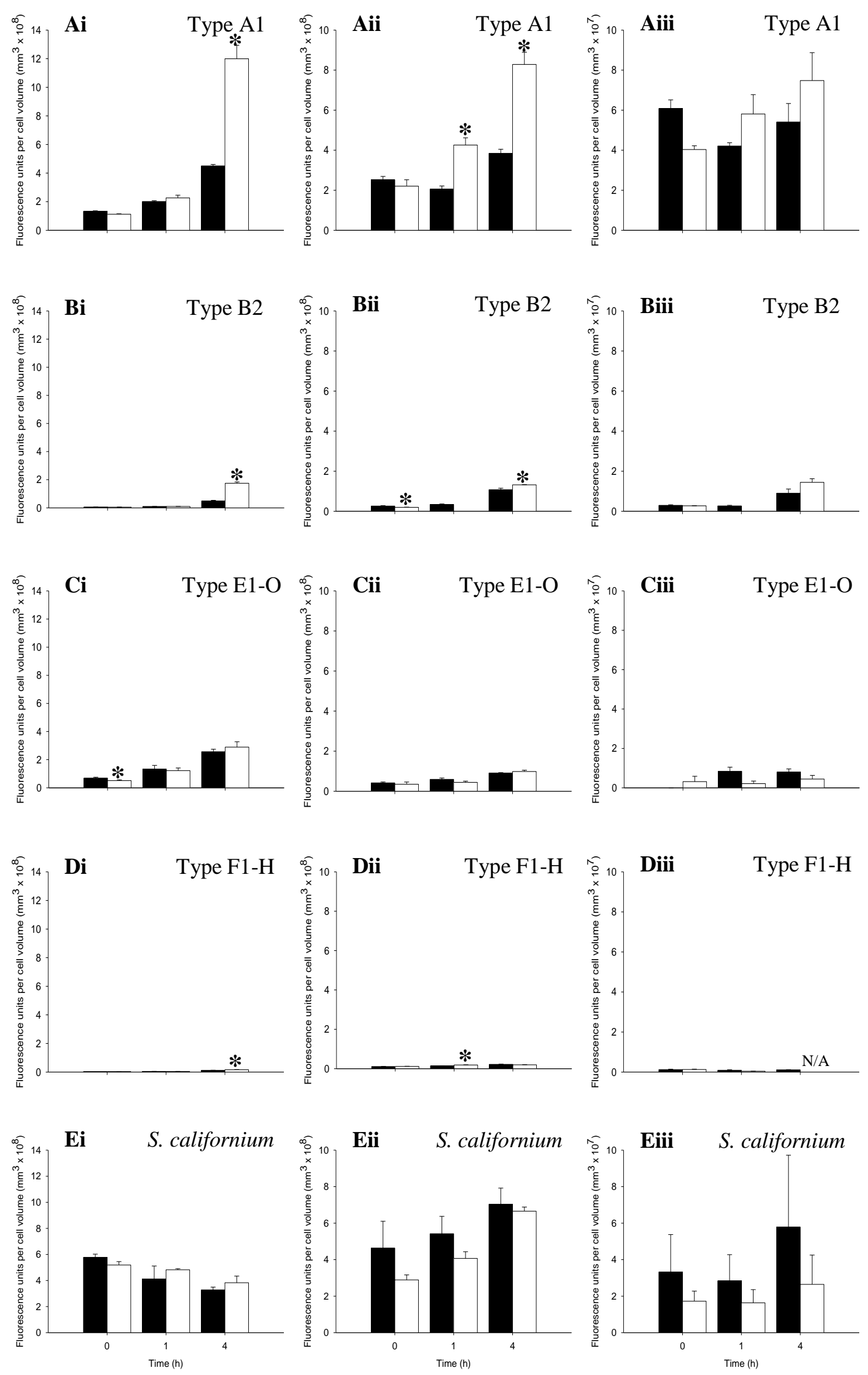
Figure 3.2 (pg. 68): Reactive oxygen species (ROS) production over time by five different Symbiodinium types when exposed to elevated temperature Results presented as fluorescence units of probes labelling ROS per algal cell volume. (Ai-Ei) General ROS; (Aii-Eii) superoxide; (AiiiEiii) singlet oxygen. N/A: No measurement could be attained due to problems with flow cytometer. Asterisks represent significant differences $(\mathrm{p} \leq 0.050)$ between the $35{ }^{\circ} \mathrm{C}$ and the $26{ }^{\circ} \mathrm{C}$ treatment. Data presented as mean \pm SE; $n=3$.

Upon the addition of the second probe dihydroethidine, which measures superoxide development, there was again an overall significant time effect for all Symbiodinium types (Friedman Test, $\chi^{2}=32.0, \mathrm{df}=2, \mathrm{p}<0.001$ ). No differences between the $26{ }^{\circ} \mathrm{C}$ and $35{ }^{\circ} \mathrm{C}$ treatments were seen in Symbiodinium E1-O and S. californium (E1; Fig. 3.2Cii, Eii). Type $\mathrm{A} 1$, on the other hand, displayed differences between the $26{ }^{\circ} \mathrm{C}$ and $35{ }^{\circ} \mathrm{C}$ treatments at the 1- 4 h (Mann-Whitney U Test, $\mathrm{Z}=-1.964, \mathrm{p}<0.05$; Fig. 3.2Aii). In Symbiodinium type $\mathrm{B} 2$, fluorescence levels at $35{ }^{\circ} \mathrm{C}$ were lower than at $26{ }^{\circ} \mathrm{C}$ at the beginning of the experiment (Mann-Whitney $\mathrm{U}$ Test, $\mathrm{Z}=-1.964, \mathrm{p} \leq 0.005$ ), but 1.2 times higher than at 26 ${ }^{\circ} \mathrm{C}$ after four hours (Mann-Whitney $\mathrm{U}$ Test, $\mathrm{Z}=-1.964, \mathrm{p} \leq 0.005$; Fig. 3.2Bii). In Symbiodinium type $\mathrm{F} 1$, fluorescence levels at $35^{\circ} \mathrm{C}$ were higher than at $26{ }^{\circ} \mathrm{C}$ but only at 1 h (Mann- Whitney U Test, $Z=-1.964, \mathrm{p}<0.05$; Fig. 3.2Dii).

When measuring singlet oxygen using the fluorescent probe SOG, no differences in fluorescence between the $26{ }^{\circ} \mathrm{C}$ and $35{ }^{\circ} \mathrm{C}$ treatments were detected across the entire exposure period in all five Symbiodinium types (Friedman Test, $\chi^{2}=1.0$, df $=2, \mathrm{p}>0.05$; Fig. 3.2Aiii-Eiii).

\subsubsection{Oxidative stress experiment}

The second part of the study investigated the effects of additional $\mathrm{H}_{2} \mathrm{O}_{2}$ on intracellular oxidative stress in Symbiodinium cells (Fig. 3.3). The changes in fluorescence of the cells without added $\mathrm{H}_{2} \mathrm{O}_{2}$ (control) and with added $\mathrm{H}_{2} \mathrm{O}_{2}(8 \mathrm{mM})$ were measured via flow cytometry (Fig. 3.4A-E). When measuring general ROS, an overall significant time $\mathrm{x}$ Symbiodinium type $\mathrm{x} \quad \mathrm{H}_{2} \mathrm{O}_{2}$ concentration effect was detected (Wilk's Lambda, $\left.F_{(9,36.657)}=2.673, p<0.02\right)$. 

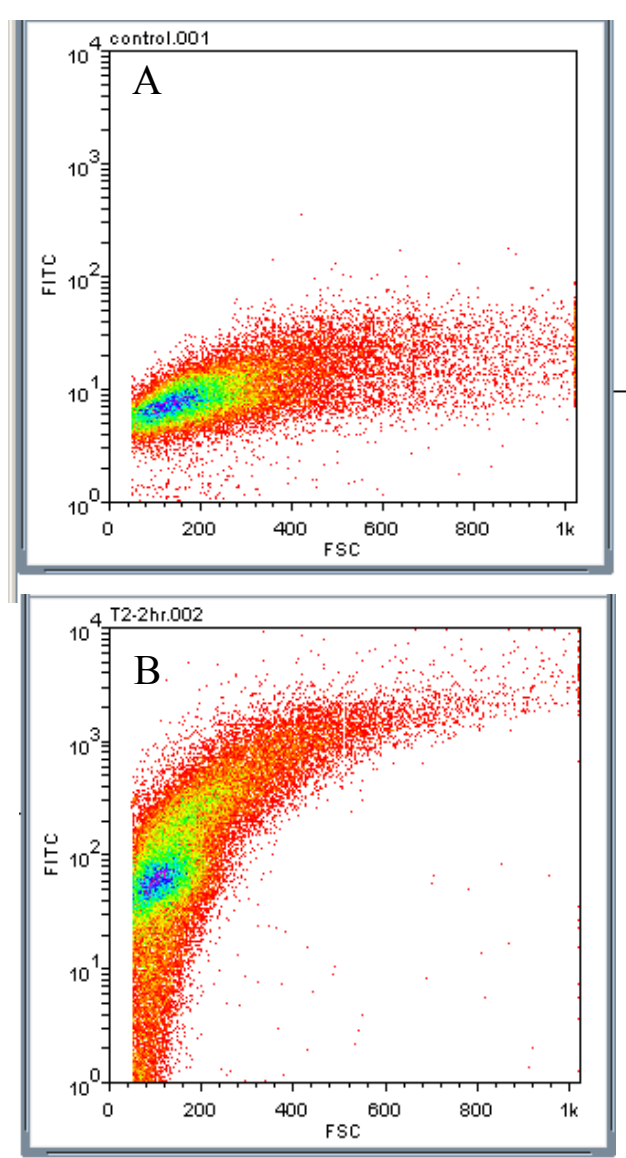

Figure 3.3: Forward scatter (FSC) of Symbiodinium cells on flow cytometer with green fluorescent probe (FITC); (A) control cells, (B) after addition of hydrogen peroxide $\left(\mathrm{H}_{2} \mathrm{O}_{2}\right)$

The fluorescence levels in the control and the $\mathrm{H}_{2} \mathrm{O}_{2}$ treatments were significantly different at all sampling points for all five Symbiodinium types (LSD, p $<0.05$ for all comparisons; Fig. 3.4Ai-Ei). However, it was not possible to determine how much the added $\mathrm{H}_{2} \mathrm{O}_{2}$ contributed to the overall measurement of general ROS tagged by the $\mathrm{H}_{2} \mathrm{DCF}-$ DA probe. In addition to the treatment effect, a temporal effect was observed. At $8 \mathrm{mM} \mathrm{H}_{2} \mathrm{O}_{2}$, fluorescence levels increased between the first measurement and all the following time points in types A1, B2 and S. californium (LSD, p $<0.03$ for all comparisons; Fig. 3.4Ai, Bi, Fi). In contrast, fluorescence levels in Symbiodinium type E1-O were 5.3 times higher at the beginning than at the end of the exposure to $\mathrm{H}_{2} \mathrm{O}_{2}$, while in type $\mathrm{F} 1$ the fluorescence level remained unchanged throughout the $\mathrm{H}_{2} \mathrm{O}_{2}$ treatment (LSD, $\mathrm{p}>0.1$; Fig. 3.4Ci, Di). Increases in fluorescence levels were also observed in the control treatment. These ranged from a doubling in $S$. californium to a six fold increase in A1 between the first and the last measurement (LSD, $\mathrm{p}<0.03)$.

With respect to superoxide, an overall significant time $\mathrm{x}$ Symbiodinium type $\times \mathrm{H}_{2} \mathrm{O}_{2}$ concentration effect was noted (Wilk's Lambda, $\mathrm{F}_{(9,34.223)}=6.053, \mathrm{p}<0.001$ ). However, no changes in fluorescence between the control and the $\mathrm{H}_{2} \mathrm{O}_{2}$ treatments were observed for Symbiodinium type A1 (Fig. 3.4Aii). In contrast, fluorescence in the control treatment was lower than in the $\mathrm{H}_{2} \mathrm{O}_{2}$ treatment at all sampling points in S. californium (LSD, p $<0.03$ for all comparisons; Fig. 3.4Fii), at $1-4 \mathrm{~h}$ in types B2 and F1 (LSD, p < 0.02 for all comparisons; Fig. 3.4Bii, Dii) and at 1- $2 \mathrm{~h}$ in Symbiodinium type E1-O (LSD, p $\leq 0.001$ for both comparisons; Fig. 3.4Cii). Again, a temporal effect was observed. Fluorescence after $4 \mathrm{~h}$ of exposure to $\mathrm{H}_{2} \mathrm{O}_{2}$ was higher than at all other time points in this treatment for 
Chapter 3
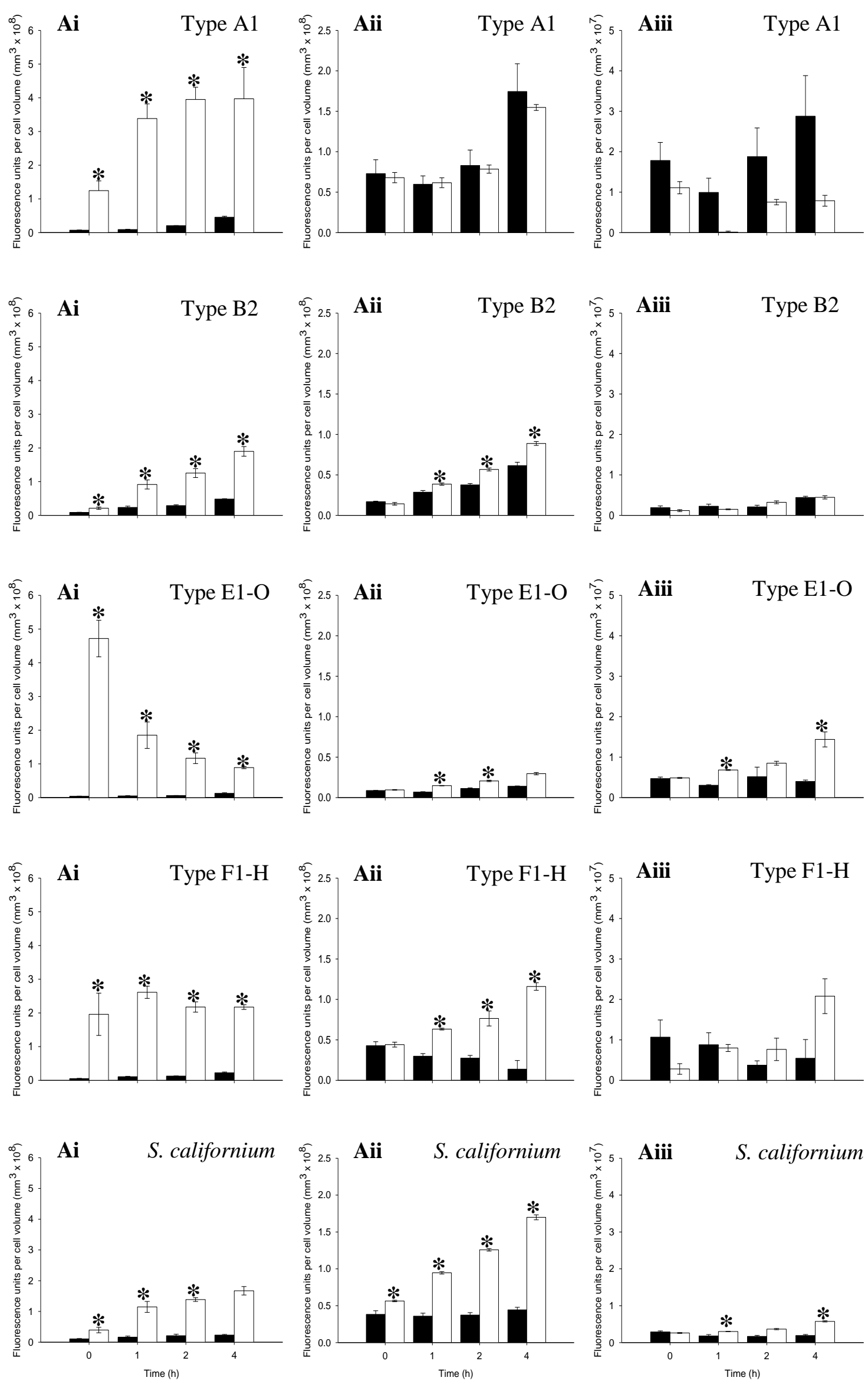
Figure 3.4 (pg. 71): Reactive oxygen species (ROS) production over time of five different Symbiodinium types when exposed to hydrogen peroxide $\left(\mathrm{H}_{2} \mathrm{O}_{2}\right)$. Results presented as fluorescent units of probes labelling ROS per algal cell volume. (Ai-Ei) General ROS; (Aii-Eii) superoxide; (Aiii-Eiii) singlet oxygen. Asterisks represent significant differences $(\mathrm{p} \leq 0.050)$ between the $8 \mathrm{mM}$ and the $0 \mathrm{mM} \mathrm{H}_{2} \mathrm{O}_{2}$ treatment. Data presented as mean $\pm \mathrm{SE} ; \mathrm{n}=3$.

all Symbiodinium types (LSD, $\mathrm{p} \leq 0.005$ for all comparisons), with a maximum six-fold increase from the initial measurement in type B2 (Fig. 3.4Bii). With the exception of $S$. californium, fluorescence also increased in the control for all types between the first and the last time points (LSD, $\mathrm{p}<0.015$ for all comparisons).

There was a significant overall effect of time x Symbiodinium type $\mathrm{x}_{2} \mathrm{O}_{2}$ concentration when measuring singlet oxygen with the probe SOG (Wilk's Lambda, $\mathrm{F}_{(6,28)}=47.077$, $\mathrm{p}<0.001)$. However, there were no significant differences between the control and the $\mathrm{H}_{2} \mathrm{O}_{2}$ treatments for Symbiodinium types A1, B2 and F1 (Fig. 3.4Aiii, Biii, Diii). The E1 Symbiodinium type showed higher fluorescence in the $\mathrm{H}_{2} \mathrm{O}_{2}$ treatment than in the control after 4 hours exposure (LSD, p $<0.005$ for both comparisons); additionally fluorescence at $8 \mathrm{mM} \mathrm{H}_{2} \mathrm{O}_{2}$ was higher than in the control at $2 \mathrm{~h}$ in $S$. californium (LSD, $\mathrm{p}<0.015$; Fig. 3.4Fiii) and at $1 \mathrm{~h}$ in type E1-O (LSD, p < 0.001; Fig. 3.4Ciii). Additionally, a temporal effect of the $\mathrm{H}_{2} \mathrm{O}_{2}$ treatment was observed. When exposed to $\mathrm{H}_{2} \mathrm{O}_{2}$, fluorescence of the E1 type (E1-O and S. californium) was higher at the end of the $4 \mathrm{~h}$ incubation than at any other time-points (LSD, $\mathrm{p}<0.005$ for all comparisons), increasing two(S. californium) to almost three-fold (E1-O) during the experiment.

\subsection{Discussion}

In this study, the effects of two stressors (increased seawater temperature and elevated concentration of $\mathrm{H}_{2} \mathrm{O}_{2}$ ) on the production of different ROS in various Symbiodinium types were investigated. After verifying the uptake of the probes via confocal microscopy, three fluorescent probes and flow cytometry were used to quantify ROS generation inside the Symbiodinium cells. I expected to find type-specific responses to each stress, and similar susceptibilities to both stressors within each Symbiodinium type. However, while responses 
to both stressors did indeed differ between the Symbiodinium types, susceptibility to one stressor did not predict the response to the other by an individual type.

Firstly, the effects of thermal stress on ROS development were measured. While no parameter of photosynthetic performance was measured, I observed that in most Symbiodinium types (E1-O, F1, S. californium) an increase in temperature did not lead to an increase in any of the measured ROS. As the probe $\mathrm{H}_{2}$ DCF-DA is very general, measuring oxygen species like $\mathrm{O}_{2}{ }^{-},{ }^{1} \mathrm{O}_{2}, \mathrm{H}_{2} \mathrm{O}_{2}, \mathrm{OH}^{\bullet}$ and various peroxides (Eruslanov and Kusmartsev 2010), it appears that intracellular oxidative stress was indeed not occurring. This might indicate that any developing intracellular stress was successfully counteracted by defence mechanisms such as antioxidants, which will be discussed later, or that a rise in temperature had no impact on the physiological properties of these Symbiodinium types. An explanation for this behaviour might be that Symbiodinium types used for the experiment had been kept under artificial laboratory conditions for years, which might have led to the cells acquiring a certain tolerance or hardiness for increased stress (Lakeman et al. 2009). Isolated Symbiodinium cells have also been shown to have a higher tolerance towards temperature stress than cells in hospite (Ralph et al. 2001).

Still, two Symbiodinium types, A1 and B2, displayed an increased ROS and superoxide production in the temperature treatment. This could indicate that they are more susceptible to this stress, with the high temperature damaging their photosystems. A subsequent buildup of excess electrons leads to the generation of general ROS. This has also been observed in two Symbiodinium types isolated from Caribbean corals, in which the type more susceptible to an increase in temperature, showed an increased $\mathrm{H}_{2} \mathrm{O}_{2}$ production (Suggett et al. 2008).

However, none of the Symbiodinium types produced more ${ }^{1} \mathrm{O}_{2}$ under stress. Therefore, it seems that thermal stress did not lead to a formation of triplet chlorophyll in PSII, the starting point for ${ }^{1} \mathrm{O}_{2}$ production (Lesser 2006). 
Negative effects of thermal stress on algae have been observed in many studies; these effects include physio-morphological changes (Lanza and Cairns 1972, Ainsworth et al. 2008) and decreases in photosynthetic efficiency (Fitt et al. 2001, Warner et al. 2002, Fine et al. 2005, Fisher 2006) and -rate (Iglesias-Prieto et al. 1992) at higher temperature. Subsequently, differences in thermal stress responses in distinct Symbiodinium types such as those described here, have been observed in other studies. Amongst others, distinct $\mathrm{C}$ types displayed different thermal susceptibilities, possibly due to varying repair mechanisms which could prevent ROS generation (Sampayo et al. 2008, Fitt et al. 2009); while Robison and Warner (2006) found that distinct Symbiodinium types adjusted to thermal stress in different ways, such as by a decline of PSII activity or cellular growth.

Secondly, oxidative stress in the Symbiodinium cells was induced by adding hydrogen peroxide. In the $\mathrm{H}_{2} \mathrm{O}_{2}$ treatment, I found higher overall concentrations of ROS, including $\mathrm{H}_{2} \mathrm{O}_{2}$, than in the control in all Symbiodinium types; this was at least partly due to the exogenous supply which was taken up by the algal cells from the medium (Downs et al. 2002). Still, Symbiodinium types E1-O and F1 appeared to respond to the added $\mathrm{H}_{2} \mathrm{O}_{2}$ faster than the other types, with higher overall ROS levels in the treatment than in the control from the start. A possible explanation is thinner cell walls (Wakefield et al. 2000). ROS levels in these two Symbiodinium types did not increase over the course of the exposure period like in the other types; rather, levels did not change (F1) or even decreased (E1-O). In both Symbiodinium types, the generation of antioxidants could be an explanation for this development (Lesser 2006). Other types such as Symbiodinium B2 seemed to take up $\mathrm{H}_{2} \mathrm{O}_{2}$ more slowly over time, possibly due to a thicker cell wall (Lesser and Shick 1989a, Wakefield et al. 2000). As a result, they might also have a slower antioxidant response (McGinty et al. 2012). This observation suggests the possibility of an inverse relationship between pro-oxidant uptake and antioxidant production and elicits further examination.

$\mathrm{O}_{2}^{-}$levels increased in all but one Symbiodinium type (A1). This RO species could have either increased due to an inhibition of its reducing agent SOD by downstream $\mathrm{H}_{2} \mathrm{O}_{2}$ (Cheng and Song 2006), or via the Mehler reaction and photosystem I (Badger et al. 2000, Tchernov et al. 2004). Type A1 showed no difference in $\mathrm{O}_{2}^{-}$with and without the addition 
Chapter 3

of $\mathrm{H}_{2} \mathrm{O}_{2}$, despite a strong increase in overall ROS concentration over time. This could indicate that this Symbiodinium type is tolerant towards $\mathrm{H}_{2} \mathrm{O}_{2}$ and oxidative stress. Antioxidants, especially superoxide dismutase, could have removed $\mathrm{O}_{2}{ }^{-}$produced as a result of $\mathrm{H}_{2} \mathrm{O}_{2}$ addition, and kept it at a level similar to that in the control, while at the same time adding to the existing $\mathrm{H}_{2} \mathrm{O}_{2}$ pool.

In contrast, only Symbiodinium type E1 (E1-O and S. californium), generated ${ }^{1} \mathrm{O}_{2}$ as a consequence of $\mathrm{H}_{2} \mathrm{O}_{2}$ addition. In this type, increasing $\mathrm{H}_{2} \mathrm{O}_{2}$ and $\mathrm{O}_{2}^{-}$levels inside the algal cells must have led to a build-up of triplett oxygen in PSII and were not counteracted by antioxidants.

Oxidative stress and the generation of ROS have been observed in human cells (Sies 1997), and higher plant- and algal-cells (Allen 1995, Alscher et al. 1997, Niyogi 1999, Ledford and Niyogi 2005); as in this study, the probe $\mathrm{H}_{2}$ DCF-DA has often been used to track general ROS development in such cells and their movement across cell barriers (Lesser 1996, Allan and Fluhr 1997, Wrona and Wardman 2006, Eruslanov and Kusmartsev 2010, Wang et al. 2011). As in thermal stress studies, different susceptibilities to ROS have been observed: Baroli et al. (2004) found that Chlamydomonas cells produced different ROS at various light levels, while a thermally tolerant Symbiodinium type produced less $\mathrm{H}_{2} \mathrm{O}_{2}$ than a sensitive one (Suggett et al. 2008, McGinty et al. 2012). However, most studies have focused on the differences in ROS neutralising antioxidant responses between algal types (Choo et al. 2004, Griffin and Bhagooli 2004, Yakovleva et al. 2004).

This is one of the first studies that look at differences in ROS generation as a consequence of stress between distinct algal types. Thermal and oxidative stress can lead to the generation of ROS via photosystem damage and various reducing agents. In the case of the thermal stress, $\mathrm{O}_{2}{ }^{-}$is first generated due to damage in the photosystems via the Mehler reaction (Allen 1997, Venn et al. 2008). Subsequently, $\mathrm{O}_{2}^{-}$is reduced to $\mathrm{H}_{2} \mathrm{O}_{2}$ by the antioxidant SOD (Halliwell and Gutteridge 1989, Alscher et al. 2002). At the same time, the presence of $\mathrm{H}_{2} \mathrm{O}_{2}$ could inhibit the SOD activity, leading to more $\mathrm{O}_{2}{ }^{-}$in return (Cheng and Song 2006). 
By adding $\mathrm{H}_{2} \mathrm{O}_{2}$ to induce oxidative stress, the processes are reversed. The $\mathrm{H}_{2} \mathrm{O}_{2}$ will have an immediate inhibitory effect on SOD, leading to an increase in $\mathrm{O}_{2}{ }^{-}$as it is no longer reduced (Cheng and Song 2006). Only as a secondary effect will $\mathrm{H}_{2} \mathrm{O}_{2}$ cause an impairment of the photosystems (Venn et al. 2008). Overall, the results in this study point towards an initial stress response defined by a generation of ROS including $\mathrm{O}_{2}{ }^{-}$and $\mathrm{H}_{2} \mathrm{O}_{2}$ rather than ${ }^{1} \mathrm{O}_{2}$.

To prevent the negative effects of photosystem damage by thermal stress, algal cells possess different protective mechanisms. Non-photochemical quenching (NPQ) dissipates excess photosynthetic energy as heat (Müller et al. 2001, Hill et al. 2005) while mycosporine-like amino acids (MAAs) specifically dissipate UV energy (Black et al. 1995, Shick and Dunlap 2002). Thermotolerance is also influenced by the fatty acid composition of the dinoflagellate's thylakoid membranes (Tchernov et al. 2004) and photosystem repair rates, such as of the important D1 protein in PSII (Takahashi et al. 2008).

In contrast, the generation of ROS is counteracted by the production of various antioxidants. $\mathrm{O}_{2}^{-}$is reduced by SOD to $\mathrm{H}_{2} \mathrm{O}_{2}$ which is subsequently reduced to water and oxygen by CAT and APX (Alscher et al. 1997, Asada 2000). ${ }^{1} \mathrm{O}_{2}$ is thought to be the most damaging ROS responsible for the loss of photosystem II (PSII) activity but it can be quenched by several non- enzymatic antioxidants, $\beta$-carotene and $\alpha$-tocopherol (Vitamin E; Venn et al. 2008) as well as glutathione (GSH; Lesser 2006; for a more comprehensive overview of various protective mechanisms, see Chapter 1).

All of these antioxidants can reduce ROS to harmless compounds and prevent further damage to cell organelles. Therefore, a Symbiodinium type's ability to generate antioxidants will determine type-specific oxidative stress susceptibilities. For instance, the lack of change in ROS levels in Symbiodinium types E1-O and F1 in the $\mathrm{H}_{2} \mathrm{O}_{2}$ treatment might have been a consequence of efficient antioxidant generation, as mentioned earlier. APX or CAT with their ability to reduce $\mathrm{H}_{2} \mathrm{O}_{2}$ (Alscher et al. 1997, Asada 2000) are more likely to have been present and responsible for a decrease in overall ROS (Venn et al. 2008). Also, as $\mathrm{H}_{2} \mathrm{O}_{2}$ was the first stressor, removing it from the cells would probably be a 
Chapter 3

priority. The production of antioxidants that reduce ROS other than $\mathrm{H}_{2} \mathrm{O}_{2}$ might have occurred more slowly and at a later time.

The increased production of ROS during thermal and oxidative stress in a number of Symbiodinium types could therefore be attributed to the addition of exogenous $\mathrm{H}_{2} \mathrm{O}_{2}$, with possible inhibition of reducing agents like SOD and impairment of the alga's photosystems. An increase in ROS over time in control samples indicated that cells experienced some stress due to sample preparation and handling; this does not diminish the significance of the applied stress treatments as differences between control and treatment were observed. In contrast, no change in ROS production was observed in some Symbiodinium types under either stress. While it is possible that a longer incubation period might have ultimately impaired the photosynthetic apparatus (Robison and Warner 2006, Fisher et al. 2012) and hence induced oxidative stress, it seems more likely that production of ROS in these Symbiodinium types was more effectively countered by antioxidants than in the more sensitive types

I originally hypothesized that a Symbiodinium type which is susceptible towards temperature will most likely be sensitive towards oxidative stress as well. However, this was not the case in all Symbiodinium types. On the one hand, the E1 type (E1-O and S. californium) showed no response to an increase of temperature, but they appeared very susceptible to $\mathrm{H}_{2} \mathrm{O}_{2}$, generating all the ROS tested for. On the other hand, Symbiodinium type A1 seemed very tolerant to $\mathrm{H}_{2} \mathrm{O}_{2}$ but less so towards an increase in temperature. Therefore, the relationship between thermal and oxidative stress remains unclear and cannot be fully explained by this study. Still, a possible explanation could be that distinct Symbiodinium types invest in different protective mechanisms. Thermally tolerant type E1, for instance, could have mechanisms to better tolerate high temperatures, such as NPQ and thylakoid membrane stability, and therefore less need for oxidative stress defences such as antioxidants. This suggests that this type might suffer damage more quickly when exposed to oxidative stress. The opposite might be true for thermally sensitive Symbiodinium type A1, which could be able to deal with thermal stress and subsequent ROS generation by investing in antioxidant production. 
Chapter 3

In this study, I tested the effects of two stressors on the production of various ROS in different Symbiodinium types, and observed differences in the quality and quantity of ROS production. My results indicate that there are different intracellular mechanisms at play when Symbiodinium cells are stressed, e.g. by increasing temperatures due to climate change. Initial sites of impairment or damage appear to vary, leading to different pathways of ROS generation. The basis for these differences, as well as the poor correlation between the responses to thermal versus oxidative stress, is of importance and should be explored in future research. For example, more information is needed regarding the cellular characteristics that convey thermal-stress resistance, as well as the antioxidant networks employed by these symbiotic algae. Therefore, this work is an important step towards elucidating the processes occurring inside Symbiodinium cells during stress events such as coral bleaching. 


\section{Chapter 4: Antioxidant potential in response to oxidative stress in different Symbiodinium types: comparison of FRAP and CAA assays}

\subsection{Introduction}

Tropical coral reefs are some of the most diverse ecosystems in the world, founded on the scleractinian (stony) corals as reef-builders and their successful association with dinoflagellates (Hoegh-Guldberg et al. 2007). These algae of the genus Symbiodinium spp. live as endosymbionts inside the coral and other cnidarian hosts, and contribute significantly to the productivity, survival and success of their hosts (LaJeunesse 2002, Hoegh-Guldberg et al. 2007). Once thought to be one pandemic species, the genus Symbiodinium has since been divided into nine genetically distinct lineages, called clades A-I, with a high number of sub-clades or 'types' (LaJeunesse 2001, Coffroth and Santos 2005, Pochon and Gates 2010). Even closely related types can differ in their physiological responses to abiotic and biotic factors (Sampayo et al. 2008). Coral hosts can associate with different Symbiodinium types, depending on the prevailing environmental conditions; therefore, the symbiont's physiology influences health and fitness of the holobiont, i.e. the coral host and all its symbiotic partners (Mieog et al. 2009).

However, coral reefs and their cnidarian inhabitants are in danger, in large part due to socalled coral bleaching events that have occurred in increasing frequency in recent years (Hoegh-Guldberg et al. 2007, Eakin et al. 2010, Harrison 2011). Coral bleaching describes the loss of symbionts and/or photosynthetic algal pigments from the coral host (Gates et al. 1992, Douglas 2003). The bleaching process is mainly induced by higher than normal seawater temperatures, in combination with high light intensities, as a consequence of climate change (Hoegh-Guldberg and Smith 1989, Gates et al. 1992, Hill and Ralph 2007, Ainsworth et al. 2008) As most corals already live close to their upper thermal limit, an increase of just a few degrees Celsius can have detrimental effects on their symbiotic alga's photosystems (Venn et al. 2008, Weis 2008). Elevated seawater temperatures can cause damage in the light and/or dark reactions of photosynthesis, which often leads to the excessive generation of various reactive oxygen species (ROS), such as singlet oxygen 
$\left({ }^{1} \mathrm{O}_{2}\right)$, superoxide $\left(\mathrm{O}_{2}{ }^{-}\right)$, hydrogen peroxide $\left(\mathrm{H}_{2} \mathrm{O}_{2}\right)$ and the highly reactive hydroxyl radical $\left(\mathrm{OH}^{\circ}\right.$; Lesser 2006). If ROS are not effectively removed by protective mechanisms, they will lead to intracellular oxidative stress (Lesser 2011). ROS can damage cell membranes, proteins and DNA (Lesser 1996, 1997) resulting in decreased growth, increased susceptibility to disease and dramatically increased mortality of the symbiotic partners (Weis 2008). Ultimately, if stress conditions persist, oxidative stress can lead to the expulsion of the Symbiodinium cells from the host (Tchernov et al. 2004, Smith et al. 2005).

Negative impacts of heat, light and ROS on the algal cell's structure and photosystems (Venn et al. 2008, Lesser, 2011) can be avoided or delayed by different protective mechanisms (Bhagooli et al. 2008, Middlebrook et al. 2008). Protection of the photosystems can be achieved by non-photochemical quenching (NPQ), when excess photosynthetic energy is dissipated as heat by the algae (Müller et al. 2001, Hill et al. 2005) or mycosporine-like amino acids (MAAs) absorb ultraviolet radiation (UV) and dissipate UV energy (Shick and Dunlap 2002, Shick 2004). Moreover, heat shock proteins (Hsps), which are produced in response to different stressors, maintain protein structure and cell function following stress (Black et al. 1995, Leggat et al. 2007). In contrast, subsequently produced ROS can be removed by the generation of antioxidants (Downs et al. 2002, Lesser 2006, Liñán-Cabello et al. 2009). There are a number of non-enzymatic antioxidants, which include glutathione (GSH) that reacts with ${ }^{1} \mathrm{O}_{2}, \mathrm{O}_{2}{ }^{-}$and $\mathrm{OH}^{\bullet}$ (Halliwell and Gutteridge 1990), and tocopherols that are able to quench ${ }^{1} \mathrm{O}_{2}$ and peroxides (Fridovich 1998, Lesser 2006). However, the first line of defence against ROS consists of enzymatic antioxidants such as superoxide dismutase (SOD), which reduces superoxide to hydrogen peroxide $\left(\mathrm{H}_{2} \mathrm{O}_{2}\right)$, and catalase (CAT) and ascorbate peroxidase (APX), which subsequently reduce $\mathrm{H}_{2} \mathrm{O}_{2}$ to water and oxygen (Alscher et al. 1997). Generally, the antioxidants act in concert to inactivate surplus ROS and prevent the formation of more active oxygen radicals like the hydroxyl radical $\mathrm{OH}^{\bullet}$ (Lesser 2006). Evidence of enzymatic antioxidant activity has been found in several coral studies (Griffin and Bhagooli 2004, Liñán-Cabello et al. 2009). 
Chapter 4

Many studies have examined the production of single antioxidants like SOD and CAT (Downs et al. 2002, Higuchi et al. 2008, Richier et al. 2008, Saragosti et al. 2010) or their gene expression (Császár et al. 2009) in Symbiodinium cells and their hosts. Assays measuring total antioxidant capacity have been widely used in other fields, such as food chemistry (Prior et al. 2005, Wolfe and Liu 2007), but have been largely neglected in coral research. In one of the exceptions, Griffin et al. (2006) used the ferric reducing antioxidant potential (FRAP) assay to compare total antioxidant capacity in different Pocillopora species from different sites. FRAP is a relatively inexpensive and easy assay in which the antioxidant reaction is activated by adding a ferric tripyridyltriazine (FeIII-TPTZ) complex. A coloured ferrous tripyridyltriazine (FeII-TPTZ) form is generated and the change in absorbance at $600 \mathrm{~nm}$ is compared to that of a standard, establishing the total antioxidant potential of a given sample (Ou et al. 2002). The cellular antioxidant activity (CAA) assay, on the other hand measures antioxidant capacity indirectly. It uses the cellpermeable probe 2',7'-dichlorodihydrofluorescein diacetate $\left(\mathrm{H}_{2} \mathrm{DCF}-\mathrm{DA}\right)$ which is nonfluorescent until the acetate group is removed by intracellular esterases and oxidation occurs in the presence of ROS, yielding highly fluorescent 2',7'-dichlorofluorescein (DCF; Baroli et al. 2004). Oxidation of the probe can be monitored (Wang et al. 2011) but this reaction can be counteracted by antioxidant compounds that prevent the oxidation of $\mathrm{H}_{2}$ DCF-DA, by reducing the existing ROS (Wolfe and Liu 2007). Therefore, increases in antioxidant quantity in a sample lead to increasing FRAP but decreasing CAA fluorescence values.

Measuring total antioxidant capacity of algal cells can potentially give us a quick and easy insight into their stress resistance. In this study, the FRAP and CAA assays were used to compare the total antioxidant potential of distinct Symbiodinium types experiencing oxidative stress induced by hydrogen peroxide $\left(\mathrm{H}_{2} \mathrm{O}_{2}\right)$. I hypothethised that differences in antioxidant production would be evident among the distinct Symbiodinium types, given the results from Chapter 2 and 3. The results will add to our knowledge of stress susceptibility and the bleaching response in Symbiodinium spp., and provide insight into the complex relationship between oxidative stress and antioxidant generation. 


\subsection{Materials and Methods}

\subsubsection{Symbiodinium types and cultivation}

Three different types of Symbiodinium cultures (A1, E1, F1) were used for the experiments (Table 1). For culture identification in this chapter, see Chapter 2. The different types were cultured in silica-free f/2 culture medium (NCMA, East Boothbay, Maine, USA) at $26 \pm 1{ }^{\circ} \mathrm{C}$ on a $12 \mathrm{~h}$ light: $12 \mathrm{~h}$ dark cycle for more than a year. White light at $55 \pm 5 \mu \mathrm{mol}$ photons $\mathrm{m}^{-2} \mathrm{~s}^{-1}$ was provided by Philips TL-D, $36 \mathrm{~W}$ fluorescent tubes. All experiments were conducted 4 weeks after the last sub-culturing.

\subsubsection{Genetic identification of Symbiodinium types}

DNA extraction from different Symbiodinium types followed the protocol of Stat et al. (2009) and Logan et al. (2010), except that DNA extraction was completed in five days. DNA was added to MyTaqTM mastermix (Bioline, London, UK) with Symbiodinium specific ITS2 primers (10 mM; forward primer: GTG AAT TGC AGA ACT CCG TG; reverse primer: CCT CCG CTT ACT TAT ATG CTT) at a final volume of $25 \mu \mathrm{L}$. Subsequently, a PCR was run on samples $\left(3 \mathrm{~min}\right.$ at $95^{\circ} \mathrm{C}, 28$ cycles of $15 \mathrm{~s}$ at $95^{\circ} \mathrm{C}, 15 \mathrm{~s}$ at $57^{\circ} \mathrm{C}, 10 \mathrm{~s}$ at $72{ }^{\circ} \mathrm{C}$ ). The final product was purified with Affymetrix USB ExoSAP-IT (In Vitro Technologies, Victoria, Australia) according to the manufacturer's instructions, and sequenced by Macrogen Inc. (Seoul, Korea).

Table 4.1: Symbiodinium cultures with corresponding host, geographic origin and sub-clade

\begin{tabular}{|l|l|l|l|}
\hline Culture I.D. & Host & Geographic origin* & Sub-clade \\
\hline CCMP2467 & Stylophora pistillata & Gulf of Aqaba & A1 \\
\hline A001 & Acropora sp. & Okinawa & E1 \\
\hline Mv & Montipora verrucosa & Hawaii & F1 \\
\hline
\end{tabular}

* for more detailed information about geographic origin, see Table 2.1. 
Chapter 4

\subsubsection{Sample preparation}

Symbiodinium cultures were centrifuged $\left(1900 \times g, 5 \mathrm{~min}, 26^{\circ} \mathrm{C}\right)$ and re-suspended in autoclaved filtered seawater $\left(1 \mu \mathrm{m}\right.$ FSW) with a concentration of $1-3 \times 10^{6}$ cells $\mathrm{mL}^{-1}$. Concentrations were determined by haemocytometer counts $(\times 100$ magnification, $n=6$ counts per cell suspension). Aliquots containing $270 \mu \mathrm{L}$ of cell suspension were then pipetted into $1.5 \mathrm{~mL}$ microcentrifuge tubes and $30 \mu 1 \mathrm{H}_{2} \mathrm{O}_{2}$ added at final concentrations of 1, 2 and $4 \mathrm{mM}$ to induce oxidative stress. Instead of $\mathrm{H}_{2} \mathrm{O}_{2}, 30 \mu \mathrm{L}$ FSW were added to control samples. Samples were kept at $26{ }^{\circ} \mathrm{C}$ under white $5 \mathrm{~W}$ halogen lights $(100 \mu \mathrm{mol}$ photons $\mathrm{m}^{-2} \mathrm{~s}^{-1}$; Exe Dichro-Cool ${ }^{\mathrm{TM}}$ ) for 4 and 2 hours for FRAP and CAA assays, respectively. Preparation times varied, but final antioxidant measurements for FRAP and CAA assays were performed after 4 hours exposure to $\mathrm{H}_{2} \mathrm{O}_{2}$.

\subsubsection{Ferric reducing antioxidant potential (FRAP) assay}

The protocol for the FRAP assay was adapted from Griffin and Bhagooli (2004). The FRAP reagent contained $300 \mathrm{mM}$ acetate buffer, $10 \mathrm{mM}$ 2,4,6-tripyridyl-s-triazine (TPTZ) solution and $20 \mathrm{mM} \mathrm{FeCl} 36 \mathrm{H}_{2} \mathrm{O}$, mixed in a 10:1:1 ratio just before use. For preparation of the $300 \mathrm{mM}$ acetate buffer, $3.1 \mathrm{~g}$ of sodium acetate trihydrate $\left(\mathrm{C}_{2} \mathrm{H}_{3} \mathrm{NaO}_{2} 3 \mathrm{H}_{2} \mathrm{O}\right)$ were mixed with $16 \mathrm{~mL}$ glacial acetic acid and brought to $1 \mathrm{~L}$ with distilled water, while the $10 \mathrm{mM}$ TPTZ solution was made in a $40 \mathrm{mM} \mathrm{HCl}$ solution. All chemicals were obtained from Invitrogen (Life Technologies, Mulgrave, Victoria, Australia). After mixing, the working FRAP reagent was heated to $37{ }^{\circ} \mathrm{C}$. A standard curve was generated from a range of $\mathrm{Fe}(\mathrm{II})$ concentrations $\left(\mathrm{FeSO}_{4} 7 \mathrm{H}_{2} \mathrm{O}: 50,75,100,200,500,1000 \mu \mathrm{M}\right)$, by measuring spectrophotometrically on an Anthos 2010 Microplate Reader (Biochrom Ltd., Cambridge, England). Subsequently, $150 \mu \mathrm{L}$ of the reagent were added to each well of a 96-well clear microtiter plate and a blank reading was performed at $600 \mathrm{~nm}$ on the plate reader. An aliquot of the sample $(50 \mu \mathrm{L})$ was added to each well and a second plate-reader measurement taken after 8 min. After blank correction, sample antioxidant concentrations were derived from the standard curve. Samples and standards were run in triplicates. 


\subsubsection{Cellular antioxidant activity (CAA) assay}

The protocol of Wolfe and Liu (2007) was used for the CAA assay. After the 2-hour incubation period with $\mathrm{H}_{2} \mathrm{O}_{2}$, the algal cells were centrifuged three times (1800 x $g, 5 \mathrm{~min}$, $26^{\circ} \mathrm{C}$ ). After each centrifugation step, the supernatant was replaced with $300 \mu$ sterile FSW to remove excess $\mathrm{H}_{2} \mathrm{O}_{2}$. The probe $(0.5 \mu \mathrm{L}$, stock concentration $100 \mathrm{mM})$ 2,7-dichlorodihydrofluorescein diacetate $\left(\mathrm{H}_{2} \mathrm{DCF}-\mathrm{DA}\right.$; Invitrogen, Life Technologies, Mulgrave, Victoria, Australia) was added to each sample followed by a 20 min incubation period. Sample aliquots $(300 \mu \mathrm{L})$ were then pipetted into the wells of a black 96-well plate. Once $\mathrm{H}_{2}$ DCF-DA was added to the cells, samples were kept in darkness to prevent degradation of the fluorescent probe. A fluorescence reading was performed every 2 min for one hour (emission: $538 \mathrm{~nm}$; excitation: $485 \mathrm{~nm}$ ) on an EnSpire 2300 Multilabel Reader (PerkinElmer, Waltham, MA, USA). The area under each curve was integrated and plotted as fluorescence units per total cell volume versus $\mathrm{H}_{2} \mathrm{O}_{2}$ concentration.

In preliminary experiments, the autofluorescence of Symbiodinium cells at different $\mathrm{H}_{2} \mathrm{O}_{2}$ concentrations was tested at the relevant wavelengths. As it was minimal and did not vary between treatments, it was not taken into consideration during the experiment.

\subsubsection{Statistical analysis}

Normality was tested using the Kolmogorov-Smirnov test and homogeneity of variance was tested with Levene's test. Subsequently, data obtained via FRAP and CAA assays were subjected to a two-way ANOVA $\left(\mathrm{H}_{2} \mathrm{O}_{2}\right.$ concentration $\mathrm{x}$ Symbiodinium type). Subsequent post hoc tests on differences between means were conducted for both data sets $(\mathrm{LSD}, \mathrm{p} \leq 0.05)$.

For all data analyses, PASW Statistics 18 was used (IBM SPSS, IBM Corp., Armonk, NY, USA). 
Chapter 4

\subsection{Results}

\subsubsection{Ferric reducing antioxidant potential (FRAP) assay}

When measuring antioxidant activity using the FRAP assay, no overall effect of $\mathrm{H}_{2} \mathrm{O}_{2}$ concentration was detected for all three Symbiodinium types combined (two-way ANOVA, $\left.\mathrm{F}_{(3)}=0.00, \mathrm{p}>0.05\right)$. Symbiodinium types $\mathrm{A} 1$ and $\mathrm{F} 1-\mathrm{H}$ showed similar patterns of antioxidant activity (FRAP assay values) with increasing $\mathrm{H}_{2} \mathrm{O}_{2}$ exposure (Fig. 4.1). Antioxidant activity decreased at 1 and $2 \mathrm{mM} \mathrm{H}_{2} \mathrm{O}_{2}$ relative to the control (LSD, $\mathrm{p} \leq 0.005$ for both types). In the $4 \mathrm{mM} \mathrm{H} \mathrm{O}_{2}$ treatment however, the activity in both Symbiodinium A1 and F1-H increased, being 71\% and $29 \%$ higher, respectively, than at $1 \mathrm{mM} \mathrm{H}_{2} \mathrm{O}_{2}$ (LSD, A1: $\mathrm{p} \leq 0.005, \mathrm{~F} 1: \mathrm{p} \leq 0.01$ ). FRAP values at $4 \mathrm{mM} \mathrm{H}_{2} \mathrm{O}_{2}$ were also higher than at $2 \mathrm{mM} \mathrm{H}_{2} \mathrm{O}_{2}$ in both these Symbiodinium types (LSD, $\mathrm{p}<0.015$ for both comparisons), but not higher than the control values (LSD, p > 0.05 for both comparisons). The responses of these two types were different, however, with A1 having a significantly lower FRAP value than type F1-H at $2 \mathrm{mM} \mathrm{H}_{2} \mathrm{O}_{2}$ (LSD, p < 0.001).

In contrast, antioxidant activity of Symbiodinium type E1-O, as also measured by the FRAP assay, showed the opposite trend. In this case, FRAP values initially increased by $19 \%$ between the control and $1 \mathrm{mM} \mathrm{H}_{2} \mathrm{O}_{2}$ treatments (LSD, $\mathrm{p}<0.001$ ), but then decreased

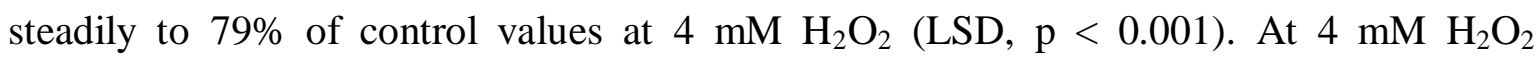
antioxidant activity was also lower than in the 1 and $2 \mathrm{mM} \mathrm{H}_{2} \mathrm{O}_{2}$ treatments (LSD, $\mathrm{p} \leq 0.001$ for both comparisons). Given this different trend, the FRAP values for Symbiodinium E1-O were significantly different from the other two Symbiodinium types at 1 and $2 \mathrm{mM} \mathrm{H}_{2} \mathrm{O}_{2}$ (LSD, $\mathrm{p}<0.01$ for all comparisons), though not at $4 \mathrm{mM} \mathrm{H}_{2} \mathrm{O}_{2}$ (LSD, $\mathrm{p}$ $>0.05$ for both comparisons) 


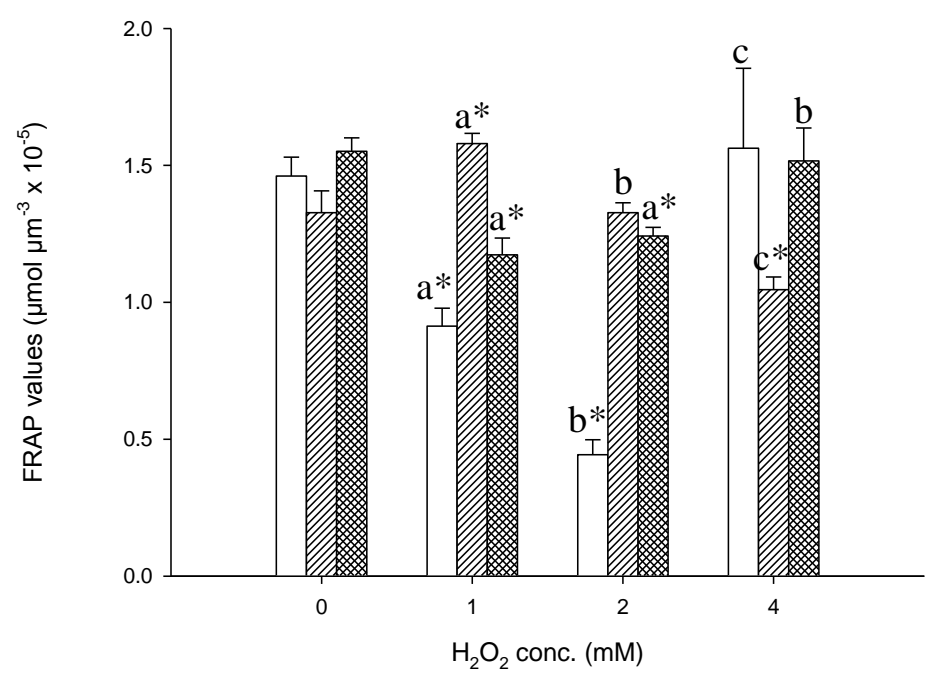

Figure 4.1: Ferric reducing antioxidant potential (FRAP) values per cell volume $\left(\mu \mathrm{M} \mu \mathrm{m}^{-3}\right)$ for three Symbiodinium types ( $\square$ : A1; $\mathbb{Z}:$ E1-O; significant differences $(\mathrm{p} \leq 0.05)$ between treatments for each species; an asterisk indicates a significant difference $(\mathrm{p} \leq 0.05)$ between the control and treatment. Means $\pm S E ; n=5$.

\subsubsection{Cellular antioxidant activity (CAA) assay}

For the CAA assay, changes in ROS concentration (and hence antioxidant activity) were measured as changes in fluorescence units over 1 hour (Fig. 4.2). Subsequently, the area under each curve was integrated (Fig. 4.3). A significant effect of both $\mathrm{H}_{2} \mathrm{O}_{2}$ concentration and Symbiodinium type on ROS concentration was observed (two-way ANOVA, $\mathrm{F}_{(4)}=$ 9.968, $\mathrm{p}<$ 0.001). Symbiodinium type A1 displayed decreasing fluorescence with increasing $\mathrm{H}_{2} \mathrm{O}_{2}$ concentration, with the value at $4 \mathrm{mM} \mathrm{H}_{2} \mathrm{O}_{2}$ being $27 \%$ lower than in the control and also significantly lower than at the other $\mathrm{H}_{2} \mathrm{O}_{2}$ concentrations (LSD, $\mathrm{p}<0.05$ for all comparisons). Fluorescence in Symbiodinium F1-H first increased with values in the $2 \mathrm{mM} \mathrm{H}_{2} \mathrm{O}_{2}$ treatment being 70 and $62 \%$ higher than in the control and the $1 \mathrm{mM} \mathrm{H}_{2} \mathrm{O}_{2}$, respectively (LSD, p < 0.001 for both comparisons). Fluorescence then declined (by 34\%) between the 2 and $4 \mathrm{mM} \mathrm{H}_{2} \mathrm{O}_{2}$ treatments (LSD, $\mathrm{p}<0.001$ ). 


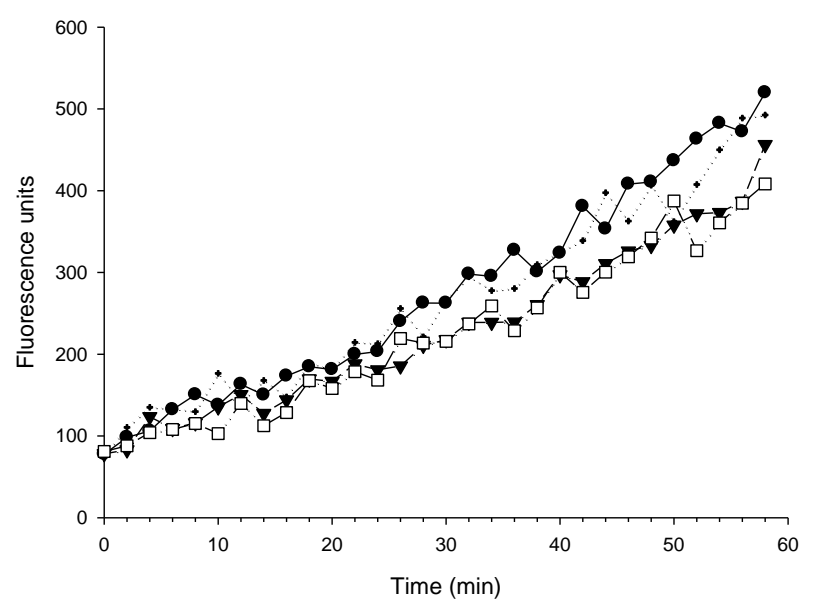

Figure 4.2: Fluorescence units for Symbiodinium type A1 after addition of different concentrations of hydrogen peroxide $\left(\mathrm{H}_{2} \mathrm{O}_{2}\right)$ and the fluorescent probe 2,7-dichlorodihydrofluorescein diacetate $\left(\mathrm{H}_{2}\right.$ DCF-DA; *: $0 \mathrm{mM}$; $:$ $1 \mathrm{mM}$; $\boldsymbol{\nabla}: 2 \mathrm{mM}$; $\square: 4 \mathrm{mM}$ ). Data are given as means $(\mathrm{n}=4)$; for clarity, no standard errors are given.

In contrast, CAA fluorescence in Symbiodinium E1-O increased consistently with increasing $\mathrm{H}_{2} \mathrm{O}_{2}$ concentration. Fluorescence values were significantly higher at 1,2 and $4 \mathrm{mM} \mathrm{H}_{2} \mathrm{O}_{2}$ than in the control (LSD, $\mathrm{p}<0.05$ for all comparisons); the value was $22 \%$ higher at $4 \mathrm{mM} \mathrm{H}_{2} \mathrm{O}_{2}$ than in the control.

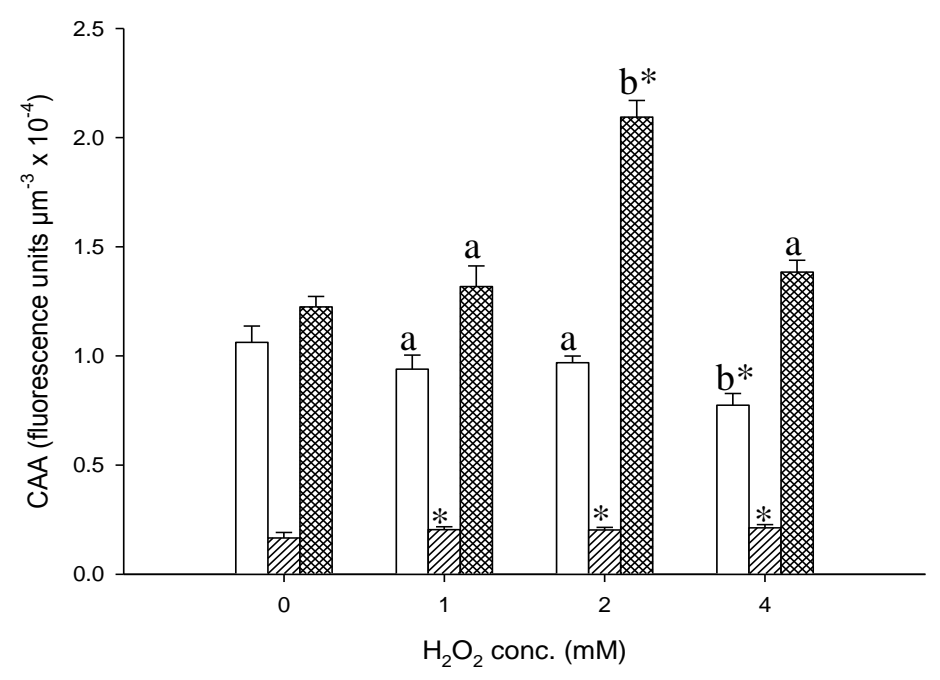

Figure 4.3: Cellular antioxidant assay (CAA) fluorescence units per cell volume for three

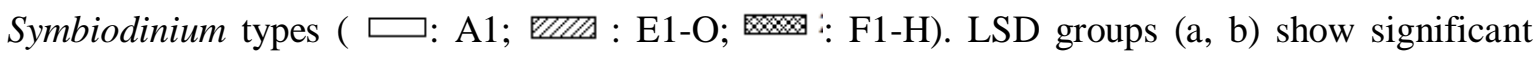
differences $(\mathrm{p} \leq 0.05)$ between treatments for each species; an asterisk highlights significant differences $(\mathrm{p} \leq 0.05)$ between the control and treatment. Measn $\pm S E ; n=4$. 


\subsection{Discussion}

The aim of this study was to measure the total antioxidant capacity in distinct Symbiodinium types exposed to different concentrations of $\mathrm{H}_{2} \mathrm{O}_{2}$. I used two different antioxidant assays, FRAP and CAA, with the FRAP assay previously having been applied to coral host tissue, but not to Symbiodinium cells. It was tested if comparable results could be produced with both assays and subsequently, if conclusions about the algal cells' stress resistance could be inferred.

Due to different methodologies, an increase in antioxidant activity is presented by increasing fluorescence in the FRAP assay and decreasing fluorescence values in the CAA. In Symbiodinium A1 and F1-H, the increase in antioxidant levels with increasing $\mathrm{H}_{2} \mathrm{O}_{2}$ concentration was matched by a reverse trend with the CAA assay, in which a decrease of fluorescence with increasing $\mathrm{H}_{2} \mathrm{O}_{2}$ concentration was observed. In contrast, antioxidant levels measured via the FRAP assay decreased in Symbiodinium type E1-O, which was corroborated by an increase in fluorescence in the CAA assay.

Symbiodinium types A1 and F1-H seem to counteract the addition of $\mathrm{H}_{2} \mathrm{O}_{2}$ by producing more antioxidants which leads to a decrease of ROS inside the cells. This might indicate that both types are relatively tolerant when facing oxidative stress caused by thermal stress in the field. A similar trend has been observed in other studies before: the antioxidants SOD, CAT and ascorbate increased during a spring bloom of the freshwater dinoflagellate Peridinium gatunense; this occurred simultaneously with elevated levels of ambient stressors such as high irradiance (Butow et al. 1997a, b). In freshly isolated Symbiodinium cells, an increase of SOD and CAT was observed at higher temperatures (Higuchi et al. 2008). Interestingly, the same study found that only CAT increased when low concentrations of $\mathrm{H}_{2} \mathrm{O}_{2}(0.3$ and $3.0 \mu \mathrm{M})$ were added to the cells. This suggests that $\mathrm{H}_{2} \mathrm{O}_{2}$ primarily affects the cell cytosol and does not necessarily induce $\mathrm{O}_{2}{ }^{-}$formation. Other studies found an increase in antioxidant concentrations in coral host tissue. CAT and glutathione-S-transferase showed a significant increase in host tissue of the coral Pocillopora capitata between winter and summer, counteracting the detrimental effects of environmental change (Liñán-Cabello et al. 2009). Overall antioxidant potential, as determined by the FRAP assay, also increased at higher water temperatures $\left(28-31^{\circ} \mathrm{C}\right)$ in 
comparison to control samples $\left(27^{\circ} \mathrm{C}\right.$ ) in two Pocillopora species (Griffin and Bhagooli 2004) Additionally, colonies that had been exposed to frequent elevated temperatures in their natural habitat displayed higher antioxidant activity than did colonies from a site with minimal thermal change. It appears that colonies from the variable site were acclimated to higher temperature and could produce a more effective antioxidant response. In contrast, Symbiodinium E1-O showed a decrease in antioxidant levels at higher $\mathrm{H}_{2} \mathrm{O}_{2}$ concentrations, which could indicate a weakening defence. This could point to a higher susceptibility to oxidative stress under natural conditions as well.

However, such results can be ambiguous and difficult to interpret. For example, decreasing FRAP values, like those in Symbiodinium type E1-O, could alternatively indicate a more efficient use of existing antioxidants (Griffin and Bhagooli 2004). Similarly, CAA data can be difficult to interpret, especially as antioxidant levels are deduced indirectly from ROS levels. Consequently, an increase in antioxidant activity in response to oxidative stress might be masked by an even greater increase in the concentration of ROS. As a consequence, it is hard to precisely deduce a Symbiodinium type's stress resistance by taking only the results from these two assays into account and they should ideally be combined with a range of other physiological measures, including photosynthetic performance.

When using the FRAP and CAA assays, several further limitations need to be taken into consideration. While results for either assay are a proxy for total antioxidant potential in a sample, slightly different antioxidant properties are measured. FRAP measures the reduction of FeIII-TPTZ to FeII-TPTZ and therefore not only quantifies antioxidants but also chemical reductants that do not act as antioxidants (Griffin and Bhagooli 2004). On the other hand, FRAP does not account for thiol antioxidants like glutathione as they are not able to reduce FeIII (Prior et al. 2005). Another consideration is the haphazard second plate-reader measurement after 8 minutes, as after adding TPTZ the absorption of the sample does not stop after several minutes but slowly increases even after several hours (Pulido et al. 2000). Because of this, FRAP values for certain compounds like ascorbic acid cannot be obtained (Huang et al. 2005), and different incubation periods will produce dissimilar results. As this study was designed to provide a snapshot of antioxidant activity 
after 4 hours exposure to $\mathrm{H}_{2} \mathrm{O}_{2}$, I maintained the 8 min incubation period. However, in future studies, kinetic absorption readings until a stable plateau is reached could be taken instead of endpoint measurements (Benzie and Strain 1996). Nevertheless, the FRAP assay as conducted here still provides important information about a cell's antioxidant defence system (Huang et al. 2005).

The CAA assay, in comparison, measures the oxidation of $\mathrm{H}_{2}$ DCF-DA to DCF by various ROS. In addition to $\mathrm{H}_{2} \mathrm{O}_{2}$, peroxynitrite $\left(\mathrm{ONOO}^{-}\right)$, nitric oxide (NO') and peroxyl radicals amongst others have been known to react with the probe (Franklin et al. 2004, Eruslanov and Kusmartsev 2010), and hence CAA is suitable for the measurement of general ROS and nitric oxygen species (NOS) levels inside cells. These different ROS can be reduced by a number of enzymatic as well as non-enzymatic antioxidants. However, one problem is that both $\mathrm{H}_{2}$ DCF-DA and DCF might not remain inside the algal cells but could leak into the medium over time; this would cause imprecision with respect to the measurement of intracellular activity (Wolfe and Liu 2007). Still, due to measuring antioxidant activity intra-cellularly, the CAA assay is an improvement on regular chemical assays (Wolfe and Liu 2007).

While both assays have their shortcomings, they corroborated one another and demonstrated that they can be applied in tandem in the study of Symbiodinium cells. In that respect, the FRAP and CAA assay present quick and relatively easy methods to gain insight into a cell's defence system. Therefore, both assays could be used as a first indicator before more expensive and time-consuming assays for single antioxidants are performed. The assays shed some light on the cellular antioxidant processes during stress giving indications of stress susceptibilities and tolerances. This could be especially useful for future research when using the assays to measure antioxidant production inside coral hosts and their symbionts under field conditions. Knowing the intracellular antioxidant activity might help to explain bleaching susceptibility of some corals and bleaching resistance of others under the same environmental conditions. In conclusion, the two antioxidant assays have excellent potential for studying the effects of various stressors, such as high temperature or high light, on Symbiodinium cells in the natural environment as well as under laboratory condition. 


\section{Chapter 5: Thermal and oxidative stress responses of different Symbiodinium types when in different states of symbiosis}

\subsection{Introduction}

Tropical coral reefs are the most diverse ecosystem in the sea; they are reliant on the symbiosis between cnidarian hosts and a diverse range of symbiotic dinoflagellate algae of the genus Symbiodinium. These algae are endosymbiotic and live in the gastrodermal cells of their coral host (Gates et al. 1992). Symbiodinium cells provide photosynthetically fixed carbon compounds to the host, while the coral host supplies inorganic nutrients like $\mathrm{CO}_{2}$ and ammonium to its algal symbionts (Gates et al. 1995). Crucially, the symbiotic algae can meet more than $100 \%$ of the host's metabolic needs under well-lit conditions (Muscatine et al. 1984, Davies 1991, Hoegh-Guldberg et al. 2007).

Coral reefs are under threat from increasing levels of natural and anthropogenic disturbance. One of the biggest threat to coral reefs today is increasing seawater temperature, as a consequence of climate change (Hughes et al. 2003, Bellwood et al. 2004). As corals live close to their upper thermal limit, a rise of just a few degrees can have negative effects on the symbiotic association (Fitt et al. 2001). The first site of impact is usually located in the symbiont's photosynthetic system (Venn et al. 2008). Three possible sites of initial damage have been suggested: the thylakoid membranes of chloroplasts (Tchernov et al. 2004) and impairment of the D1 protein in photosystem II (PSII) due to inhibition of its repair mechanisms (Warner et al. 1999, Douglas 2003, Nishiyama et al. 2006) and of the Calvin Benson cycle (Jones et al. 1998). This damage interrupts the normal flow of electrons between photosystems, and leads to an unusual build-up of energy and a reduction in photosynthetic capacity, or photoinhibition. Accumulated electrons interact with oxygen, forming reactive oxygen species (ROS) such as singlet oxygen $\left({ }^{1} \mathrm{O}_{2}\right)$ and superoxide $\left(\mathrm{O}_{2}{ }^{-}\right)$, which can be reduced to hydrogen peroxide $\left(\mathrm{H}_{2} \mathrm{O}_{2}\right)$ and the highly reduced hydroxyl radical $\left(\mathrm{OH}^{\circ}\right.$; Tchernov et al. 2004, Lesser 2006). 
ROS are constantly produced under normal, non-stressful conditions inside algal cells but are effectively removed by antioxidants (Mittler 2002, Lesser 2012). Oxidative stress occurs when the production of ROS overwhelms the antioxidant system. Antioxidants like superoxide dismutase (SOD), which reduces $\mathrm{O}_{2}{ }^{-}$to $\mathrm{H}_{2} \mathrm{O}_{2}$, and ascorbate peroxidase (APX), which reduces $\mathrm{H}_{2} \mathrm{O}_{2}$ to $\mathrm{H}_{2} \mathrm{O}$ and $\mathrm{O}_{2}$, are produced as the first line of defence (Lesser 2006, Venn et al. 2008). Other protective mechanisms that neutralize oxidative stress include fluorescent pigments, which deactivate $\mathrm{H}_{2} \mathrm{O}_{2}$ (Palmer et al. 2010), and heat shock proteins (Hsps), which help to maintain protein structure and function following stress (Black et al. 1995, Baird et al. 2009). ROS can denature proteins, oxidize cell membranes and damage DNA (Lesser 1996), and leak from the algal cell into the host cell (Lesser 2006). When oxidative stress inside the cell persists, it may lead to the end of the symbiosis (Franklin et al. 2004). The exact processes behind this are not quite clear, but possible scenarios are expulsion of Symbiodinium cells, whole host cell detachment, or apoptosis, all of which would protect the host from more damage (Gates et al. 1992, Weis 2008). Due to the loss of Symbiodinium cells and/or algal photosynthetic pigments, the underlying white calcium carbonate skeleton becomes apparent through the coral tissue, leading to the well-known phenomenon of coral bleaching (Gates et al. 1992, Hoegh-Guldberg 1999, HoeghGuldberg et al. 2007). Bleaching results in decreased growth, fecundity and increased susceptibility to disease in the host, often followed by death (Richier et al. 2008, Weis et al. 2008).

While the phenomenon of bleaching is seen across the Symbiodinium genus, the thermal threshold and rate of response can differ greatly. Symbiodinium is a very diverse genus with nine clades and many sub-clades, or 'types' (Coffroth and Santos 2005, Pochon and Gates 2010). Even though types might be very similar genetically, their response to the same stress conditions can vary widely (Sampayo et al. 2008). An increased tolerance to stress in a Symbiodinium type can partly be explained by the symbiont's defence system (Baker et al. 2004, Warner et al. 2006, Hill and Ralph 2008, Suggett et al. 2008, also Chapter 3 of this thesis). But in a healthy symbiosis, the host will not solely rely on its symbiont's protective mechanisms. Rather, both symbiotic partners have developed strategies to deal with stress, such as generation of the aforementioned antioxidants (Bhagooli et al. 2008). Therefore, when trying to define properties of stress-resistance in specific Symbiodinium sub-clades, algal cultures are a good tool. Various environmental 
effects can be isolated and these, as well as subsequent properties of stress-resistance, can be observed without host influence (Robison and Warner 2006). However, for a more complete and realistic picture, studies need to be done with algal cells inside the host, i.e. in hospite (Fitt et al. 2009). Only by doing this one can account for the host's role.

In this study, I tested the effects of thermal and oxidative stress, induced by hydrogen peroxide $\left(\mathrm{H}_{2} \mathrm{O}_{2}\right)$, on three different Symbiodinium types in hospite in corals and when freshly isolated. I measured changes in photophysiological parameters as well as oxidative stress resistance in the form of antioxidant production. The relationship between thermal and oxidative stress was explored and the influence of the host on both was investigated.

\subsection{Material and Methods}

\subsubsection{Collection of corals}

Fragments of five scleractinian coral species were collected on the reef flat of Heron Island (HI; Great Barrier Reef, 23 26' $60 \mathrm{~S}, 151^{\circ} 55^{\prime} 00 \mathrm{E}$, Australia) during low tide in November/December 2010. The species Pocillopora damicornis, Porites cylindrica, Isopora palifera, Acropora millepora and Montipora digitata were chosen as they are commonly associated with different Symbiodinium types (C1, C3, C15; Table 5.1); this allowed for comparison between different types of clade C Symbiodinium both in different and the same host species. For each coral species, fragments were collected from five different colonies $(n=5)$. After collection, coral explants for thermal stress experiments were mounted onto PVC rings (diameter: $2 \mathrm{~cm}$, width: $1 \mathrm{~cm}$ ) with two-part epoxy putty ('Knead It Aqua', Selleys Australia). In addition, coral fragments were hung on bamboo sticks with fishing line for subsequent oxidative stress experiments or experiments with freshly isolated Symbiodinium cells. They were acclimatized for a minimum of three days in glass aquaria with an open flow-through system with water from the surrounding lagoon $\left(26 \pm 1{ }^{\circ} \mathrm{C}\right)$. The aquaria were covered with mesh to shield them from direct sunlight, giving a peak irradiance of $200 \mu \mathrm{mol}$ photons $\mathrm{m}^{-2} \mathrm{~s}^{-1}$ over the daily light cycle. 


\subsubsection{Thermal stress experiment with Symbiodinium sp. in hospite}

For holobiont temperature experiments, equal numbers $(n=30)$ of coral explants of P. damicornis, P. cylindrica, I. palifera, A. millepora and M. digitata were distributed into three different aquaria in the flow-through system described above. After three days of acclimatization, the temperature in two of the tanks was raised to $28.5 \pm 1{ }^{\circ} \mathrm{C}$ and $31 \pm 1{ }^{\circ} \mathrm{C}$, respectively, with submersible aquarium heaters. The remaining tank was maintained at the control temperature, which was that of the surrounding lagoon water $\left(26 \pm 1{ }^{\circ} \mathrm{C}\right)$. Coral samples were maintained at these temperatures for six days, during which time physiological measurements were taken. Using pulse amplitude modulated fluorometry (Diving PAM, Walz, Germany) measurements were taken of the dynamic $\left(\Delta \mathrm{F} / \mathrm{F}_{\mathrm{m}}{ }^{\prime}\right)$ yield, followed by the maximum fluorescent $\left(\mathrm{F}_{\mathrm{v}} / \mathrm{F}_{\mathrm{m}}\right)$ yield of PSII after $20 \mathrm{~min}$ dark adaption, at $1 \mathrm{pm}$, and 1-2 hours after sunset (8-9 pm). Before turning the heaters on in the morning of day 1 , and at the end of days 2 and 6 , ten explants per coral species and treatment were taken from each tank for further analyses, including antioxidant measurements.

Table 5.1: Symbiodinium sub-clades present in five coral species from Heron Island. ITS sub-clade was determined using single stranded conformation polymorphism (SSCP). Samples were selected for sequencing and aligned to known ITS2 sequences using the GeoSymbio Database and Geneious.

\begin{tabular}{|l|l|l|l|l|}
\hline Host & Sub-clade & $\begin{array}{l}\text { Geneious } \\
(\% \text { agreement })\end{array}$ & GenBank & $\begin{array}{l}\text { Sequence } \\
\text { length }\end{array}$ \\
\hline Pocillopora damicornis & $\mathrm{C} 1 / \mathrm{C} 1 \mathrm{c}$ & $100 \% / 99.6 \%$ & EU449103 & 283 \\
\hline Acropora millepora & $\mathrm{C} 3$ & $100 \%$ & AF499789 & 283 \\
\hline Isopora palifera & $\mathrm{C} 3 *$ & N/A & N/A & N/A \\
\hline Montipora digitata & $\mathrm{C} 15$ & $100 \%$ & AF499789 & 283 \\
\hline Porites cylindrica & $\mathrm{C} 15$ & $100 \%$ & AY239369 & 283 \\
\hline
\end{tabular}

* Symbiodinium type from host I. palifera is C3 with little variation according to the literature (Benzie et al. 1995, LaJeunesse et al. 2003, LaJeunesse et al. 2004); this was not confirmed here due to problems with sample preservation. 
Chapter 5

\subsubsection{Oxidative stress experiment with Symbiodinium sp. in hospite}

Additionally, the effects of oxidative stress induced by hydrogen peroxide $\left(\mathrm{H}_{2} \mathrm{O}_{2}\right)$ on Symbiodinium in hospite were tested. On the experimental day, equal numbers of coral explants (total $n=10$ ) of each of the five coral species were transferred from the holding tanks into four small plastic containers filled with seawater (volume $0.7 \mathrm{~L}$ ) from the surrounding reef. $35 \% \mathrm{H}_{2} \mathrm{O}_{2}(\mathrm{v} / \mathrm{v})$ was added to three of the containers to give final concentrations of 10,20 and $40 \mathrm{mM}$. These $\mathrm{H}_{2} \mathrm{O}_{2}$ concentrations were chosen as preliminary experiments showed that they elicited a full range of responses in Symbiodinium cells in hospite. One container was kept as a control and held seawater only. The same physiological measurements were performed as described for the thermal stress experiment. Diving PAM measurements were performed prior to the addition of $\mathrm{H}_{2} \mathrm{O}_{2}$, and after 1 and 2 hours of exposure $(n=5)$. Temperature was maintained at $26 \pm 1{ }^{\circ} \mathrm{C}$ within experimental chambers by pumping lagoon water around the outside of the containers throughout the experiment. Two explants per replicate coral and $\mathrm{H}_{2} \mathrm{O}_{2}$ treatment were used for follow-up analyses after the last PAM measurement.

\subsubsection{Thermal and oxidative stress experiments with freshly isolated Symbiodinium sp. (FIS)}

To isolate potential host effects, freshly isolated Symbiodinium (FIS) from the corals P. cylindrica and I. palifera were also exposed to elevated temperature and $\mathrm{H}_{2} \mathrm{O}_{2}$. Attempts at obtaining healthy freshly isolated cells from other coral host species were unsuccessful, as demonstrated by their very poor photosynthetic performance $\left(\mathrm{F}_{\mathrm{v}} / \mathrm{F}_{\mathrm{m}}<0.1\right.$; data not shown). Coral tissue was carefully removed from the skeleton in $25-\mu \mathrm{m}$ filtered seawater (FSW) with a toothbrush. Subsequently, animal and algal cells were separated by three centrifugation cycles $\left(800 \times g, 5 \mathrm{~min}, 25^{\circ} \mathrm{C}\right)$, with the supernatant being replaced each time with FSW. After the last cycle, cells were concentrated to $2.5 \times 10^{6}$ cells $\mathrm{mL}^{-1}$; concentrations were determined by haemocytometer counts $(\times 100$ magnification, $n=6$ counts per cell suspension). 


\section{Chapter 5}

For the thermal stress experiment, $200 \mu \mathrm{L}$ of each cell suspension were pipetted into the wells of white 96-well plates (BD Falcon ${ }^{\mathrm{TD}}$, USA). Well plates were placed in plastic containers partly submerged into three glass aquaria. The control was maintained at the ambient lagoon water temperature of $26 \pm 1{ }^{\circ} \mathrm{C}$, and the two experimental tanks were heated to $28.5 \pm 1{ }^{\circ} \mathrm{C}$ and $31 \pm 1{ }^{\circ} \mathrm{C}$ with aquarium heaters. For the oxidative stress experiment, $180 \mu \mathrm{L}$ of cell suspension were pipetted into the wells of white 96 - well plates. $\mathrm{H}_{2} \mathrm{O}_{2}$ was added to the cells to obtain final concentrations of $0,1,2$ and $4 \mathrm{mM}$ and a total volume of $200 \mu \mathrm{L}$. Temperature was kept constant at $26 \pm 1{ }^{\circ} \mathrm{C}$ via the flow-through system. In both experiments, diving PAM measurements were taken as described previously, and were performed before the start of the experiments, i.e. before placing the cells into different temperatures or adding $\mathrm{H}_{2} \mathrm{O}_{2}$, and at one hour intervals thereafter.

\subsubsection{Chlorophyll $a$ and $c_{2}$}

Half of the coral explants collected during the in hospite thermal and oxidative stress experiments were removed using directional high-pressured air ('airbrushing') on Heron Island ( $\mathrm{n}=5$ per species, treatment and measuring point). Each of the resulting homogenates was made up to a volume of $20 \mathrm{~mL}$ with FSW. A sub-sample $(1 \mathrm{~mL})$ of the homogenate was taken to determine Symbiodinium cell density. The remaining homogenate was centrifuged at high speed $(16,000 \times \mathrm{g}, 5 \mathrm{~min})$ to pellet the Symbiodinium cells. The supernatant was discarded and the algal cells re-suspended in $2 \mathrm{~mL}$ acetone and maintained at $4{ }^{\circ} \mathrm{C}$ for 24 hours in the dark to extract photosynthetic pigments. Chlorophyll $a$ and $c_{2}$ concentrations of the extract were then determined using the method of Jeffrey and Humphrey (1975).

The surface area of each coral explant skeleton was established with the paraffin wax method (Stimson and Kinzie 1991). These values were used to calculate symbiont density and chlorophyll concentrations per unit surface area. 
Chapter 5

\subsubsection{Antioxidant assays}

Superoxide dismutase assay

The superoxide dismutase (SOD) assay was run at Victoria University of Wellington, NZ, with the remaining coral explants collected during the in hospite experiments $(\mathrm{n}=5$ per species, treatment and measuring point). Coral explants were brought from Heron Island to $\mathrm{NZ}$ on dry ice and were maintained before and after at $-80{ }^{\circ} \mathrm{C}$.

The SOD assay was adapted from Beyer and Fridovich (1987) and Janknegt et al. (2007). In this method, the dye NitroBlue Tetrazolium chloride (NBT) competes with the sample's SOD for $\mathrm{O}_{2}^{-}$generated by riboflavin under illumination. The presence of SOD inhibits the reduction of NBT and prevents a colour change. Algal cells were isolated from the coral host by airbrushing and centrifugation $\left(5000 \mathrm{x} g, 2 \mathrm{~min}, 4^{\circ} \mathrm{C}\right)$, the host supernatant was discarded, and the algal cells were re-suspended in $300 \mu \mathrm{L} 50 \mathrm{mM} \mathrm{KH} \mathrm{PO}_{4} / 0.1 \mathrm{mM}$ ethylenediaminetetraacetic acid (EDTA) buffer. Re-suspended algal cells were homogenized using the TissueLyser LT bead mill (Qiagen, NZ, $50 \mathrm{~Hz}, 5 \mathrm{~min}$ ), followed by further centrifugation $\left(16,000 \times \mathrm{g}, 10 \mathrm{~min}, 4^{\circ} \mathrm{C}\right)$. The supernatant was carefully transferred into a new tube without disturbing the pellet. An aliquot $(50 \mu \mathrm{L})$ of each sample was added to $120 \mu \mathrm{L}$ of $0.01 \mathrm{M} \mathrm{NBT/1 \%}$ Triton X-100 in buffer (0.05 $\mathrm{M} \mathrm{KH}_{2} \mathrm{PO}_{4} / 0.0001 \mathrm{M}$ EDTA) in the wells of a 96-well plate. Next, $130 \mu \mathrm{L}$ of $0.25 \mathrm{M}$ L-methionine in $0.15 \mathrm{mM}$ riboflavin were pipetted into the wells. Both pipetting steps were done in low light to prevent an early generation of $\mathrm{O}_{2}^{-}$. Afterwards, the well plate was placed under four fluorescence tubes (Philips TLD/18 W, $30 \mathrm{~cm}$ distance) at $200 \mu \mathrm{mol}$ photons $\mathrm{m}^{-2} \mathrm{~s}^{-1}$ for $10 \mathrm{~min}$ before measuring the absorbance at $560 \mathrm{~nm}$ using an EnSpire 2300 Multilabel Reader (PerkinElmer, Waltham, MA, USA). Fifty percent inhibition was calculated by regression using a natural semi-log curve generated by the standard, which consisted of SOD in ammonium sulfate with different specific activities. Established sample activities were calculated per mg algal protein as determined by the Bradford assay (see below).

Ascorbate peroxidase assay

The same coral explants were used for SOD and ascorbate peroxidase (APX) assays. APX activity was determined by adapting the method of Nakano and Asada (1981). Airbrushed 
algal cells were prepared for the assay in the same way as for the SOD test. An aliquot $(20 \mu \mathrm{L})$ of each sample and $180 \mu \mathrm{L}$ of assay buffer $(50 \mathrm{mM} \mathrm{PO} / 0.1 \mathrm{mM}$ EDTA; $0.5 \mathrm{mM}$

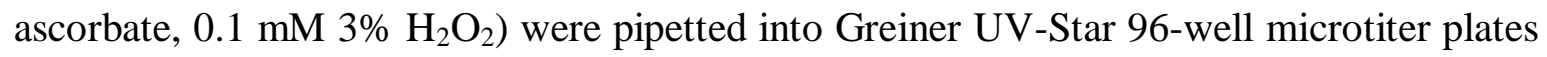
(Raylab NZ Ltd., Auckland, NZ). The reaction was started by the addition of $\mathrm{H}_{2} \mathrm{O}_{2}$ and the change in absorbance caused by peroxidation of ascorbate to dehydroascorbate was measured over $7 \mathrm{~min}$ at $290 \mathrm{~nm}$, using an EnSpire 2300 Multilabel Reader. Sample activity was determined via the Lambert-Beer Law and by using a linear part of the decrease in absorbance. Enzyme activity was calculated per $\mathrm{mg}$ algal protein as determined by the Bradford assay (see below).

\section{Bradford assay}

Protein content of the Symbiodinium cells was determined using the Bradford assay (Bradford 1976). Aliquots (10 $\mu \mathrm{L}$ and $30 \mu \mathrm{L})$ of Symbiodinium cell suspension were pipetted into the wells of a 96-well plate with $90 \mu \mathrm{L}$ and $70 \mu \mathrm{L}$ of distilled water, respectively. After adding $100 \mu \mathrm{L}$ of Bradford reagent, an absorbance reading at $595 \mathrm{~nm}$ was taken on an EnSpire 2300 Multilabel Reader. Results were compared with those of a standard curve obtained by different concentrations of bovine serum albumin in distilled water. Chemicals for the SOD, APX and Bradford assays were acquired from Sigma Aldrich New Zealand Ltd (Auckland, NZ).

Ferric Reducing Antioxidant Potential (FRAP) assay

Total antioxidant capacity of FIS was determined on Heron Island using the Ferric Reducing Antioxidant Potential (FRAP) assay. The protocol for the FRAP assay was adapted from Griffin and Bhagooli (2004). A combination of $300 \mathrm{mM}$ acetate buffer, $10 \mathrm{mM}$ 2,4,6-tripyridyl-s-triazine (TPTZ) solution and $20 \mathrm{mM} \mathrm{FeCl}_{3} 6 \mathrm{H}_{2} \mathrm{O}$ were mixed in a 10:1:1 ratio just before use to obtain the working FRAP reagent. For preparation of the $300 \mathrm{mM}$ acetate buffer, $3.1 \mathrm{~g}$ sodium acetate trihydrate $\left(\mathrm{C}_{2} \mathrm{H}_{3} \mathrm{NaO}_{2} 3 \mathrm{H}_{2} \mathrm{O}\right)$ were mixed with $16 \mathrm{~mL}$ glacial acetic acid and brought to $1 \mathrm{~L}$ with distilled water, while the $10 \mathrm{mM}$ TPTZ solution was made in a $40 \mathrm{mM} \mathrm{HCl}$ solution. All chemicals were attained from Invitrogen (Mulgrave, Victoria, Australia). After mixing, the working FRAP reagent was heated to $37^{\circ} \mathrm{C}$. Reagent $(150 \mu \mathrm{L})$ was added to each well of a 96 -well clear microtiter plate and a 
blank reading was performed at $600 \mathrm{~nm}$ on a plate reader (Biochrom Ltd., Cambridge, England). Following the initial absorbance reading, $50 \mu \mathrm{L}$ of sample were added to each well and a second plate reader reading was taken after $8 \mathrm{~min}$. The initial blank reading was subtracted from the second reading of the FRAP reagent plus sample. The change in absorbance was used to determine the sample's FRAP value by comparison with a known standard; to obtain the standard curve, different $\mathrm{Fe}(\mathrm{II})$ concentrations $\left(\mathrm{FeSO}_{4} 7 \mathrm{H}_{2} \mathrm{O}\right)$ were run on the plate reader $(50,75,100,200,500,1000 \mu \mathrm{M})$ and the resulting FRAP values were plotted against their concentrations. Both, samples and standards were run in triplicate.

\subsubsection{Genetic identification of Symbiodinium types}

Coral tissue was removed from the skeleton in the field, by airbrushing in FSW. Cells were centrifuged at high speed $\left(16,000 \times \mathrm{g}, 5 \mathrm{~min}, 25^{\circ} \mathrm{C}\right)$ and the supernatant was replaced with $\mathrm{NaCl}$-saturated $20 \%$ dimethyl sulfoxide (DMSO) and subsequently frozen at $-18{ }^{\circ} \mathrm{C}$ until processing at Victoria University of Wellington. A protocol by Logan et al. (2010) for DNA extraction was used, except that the time of extraction was adjusted to five days. DNA was added to MyTaqTM mastermix (Bioline, London, UK) with Symbiodinium specific ITS2 primers (10 mM; forward primer: GTG AAT TGC AGA ACT CCG TG; reverse primer: CCT CCG CTT ACT TAT ATG CTT) and double distilled water $\left(\mathrm{ddH}_{2} \mathrm{O}\right)$ to a final volume of $25 \mu \mathrm{L}$. A polymerase chain reaction (PCR) was run on samples (3 min at $95{ }^{\circ} \mathrm{C}, 28$ cycles of $15 \mathrm{~s}$ at $95{ }^{\circ} \mathrm{C}, 15 \mathrm{~s}$ at $57{ }^{\circ} \mathrm{C}, 10 \mathrm{~s}$ at $72{ }^{\circ} \mathrm{C}$ ). ITS sub-clade was determined using single stranded conformation polymorphism (SSCP). Formamide loading buffer (80\% formamide, $5 \mathrm{mM}$ EDTA, $10 \mathrm{mg} \mathrm{mL}^{-1}$ blue dye, $\mathrm{pH}$ 8) and DNA amplicons were mixed in equal volumes and heated to $95^{\circ} \mathrm{C}$ for $5 \mathrm{~min}$. After this, DNA was cooled for $10 \mathrm{~min}$ on ice before being loaded on to an SSPC gel (15\% polyacrylamide (50:1 acrylamide:bisacrylamide), 12\% x 5 tris-/borate/EDTA (TBE) buffer (450 mM Tris, $450 \mathrm{mM}$ boric acid and $10 \mathrm{mM}$ EDTA for x 5 stock solution), $75 \mu \mathrm{L}$ Temed $10 \%$ and

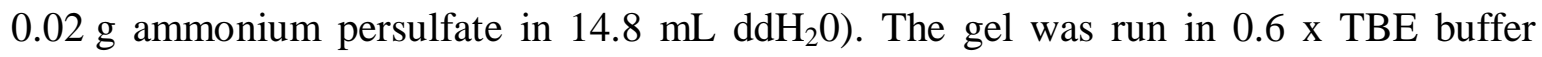
$(110 \mathrm{~V}, 150 \mathrm{~min})$ and subsequently stained with ethidium bromide in TBE buffer (final concentration $0.5 \mu \mathrm{L} \mathrm{mL}^{-1}$ ). Resulting distinct DNA bands were selected and dissolved in $\mathrm{ddH}_{2} \mathrm{O}$ overnight before being added to MyTaqTM mastermix and re-amplified via PCR ( $3 \mathrm{~min}$ at $95{ }^{\circ} \mathrm{C}, 29$ cycles of $15 \mathrm{~s}$ at $95{ }^{\circ} \mathrm{C}, 15 \mathrm{~s}$ at $56^{\circ} \mathrm{C}, 10 \mathrm{~s}$ at $72{ }^{\circ} \mathrm{C}$ ). PCR product was 
purified (Affymetrix USB ExoSAP-IT) according to manufacturer's instructions, sequenced (Macrogen Inc., Seoul, Korea) and aligned to known ITS2 sequences using the GeoSymbio Database, NCBI Genbank, and Geneious software (Geneious 5.5.3, Biomatters Ltd. Auckland, NZ). Polyacrylamide was supplied by Bio-Rad (Auckland, New Zealand), Affymetrix USB ExoSAP-IT by In Vitro Technologies (Victoria, Australia), and the remaining chemicals by Sigma Aldrich New Zealand Ltd.

\subsubsection{Statistical analysis}

Normality was tested using Shapiro Wilk's test. For normal distributed data collected over time, several repeated measure analyses of variance (rANOVA, Symbiodinium type $\mathrm{x}_{2} \mathrm{O}_{2}$ concentration over time and Symbiodinium type x temperature over time, respectively) with a Greenhouse-Geisser correction were performed. For normally distributed data that were not collected over time, two-way ANOVAs (Symbiodinium type $\mathrm{x}$ treatment) were performed. Subsequent post hoc tests were performed in both cases to test for differences of means (Tukey HSD, $\mathrm{p} \leq 0.05$ ). For non-parametric data, the Friedman Test was performed to test for any time effect. Subsequently, Mann-Whitney U Tests were performed for single time points to test for differences of means between treatments $(\mathrm{p} \leq 0.05)$. The Wilcox test was used to test for differences of means between time points ( $\mathrm{p} \leq 0.05$ ). Additionally, correlation analyses with the Pearson coefficient were run for symbiont density and chlorophyll concentrations from experiments with whole coral explants. For all data analyses, the statistical program SPSS Statistics 19 (IBM SPSS, IBM Corp., Armonk, NY, USA) was used.

\subsection{Results}

\subsubsection{Photosynthetic parameters for Symbiodinium sp. in hospite}

The maximum quantum yield $\left(\mathrm{F}_{\mathrm{v}} / \mathrm{F}_{\mathrm{m}}\right)$ decreased over time when the different Symbiodinium types were subjected to increasing temperatures in hospite (Chi-Square = 39.056, df $=5, \mathrm{p}<0.001)$. However, the different types displayed varying responses to thermal stress (Fig. 5.1). Symbiodinium type C1 in P. damicornis did not show any 
difference in $\mathrm{F}_{\mathrm{v}} / \mathrm{F}_{\mathrm{m}}$ between the control $\left(26{ }^{\circ} \mathrm{C}\right.$ ) and $28.5{ }^{\circ} \mathrm{C}$ (Mann-Whitney $\mathrm{U}, \mathrm{p}>0.005$ for all comparisons; Fig. 5.1Ai), but had no measurable $F_{v} / F_{m}$ at $31{ }^{\circ} \mathrm{C}$ after 2 days of exposure and so measurements at this temperature were discontinued. Type C3 in A. millepora displayed higher $\mathrm{F}_{\mathrm{v}} / \mathrm{F}_{\mathrm{m}}$ values in the control than in the $28.5{ }^{\circ} \mathrm{C}$ treatment after three days (Mann-Whitney $\mathrm{U}, \mathrm{Z}=-2.095$, $\mathrm{p}<0.05$ ) and also 20-100\% higher values than in the $31{ }^{\circ} \mathrm{C}$ treatment from Day 2 until the end of the experiment (Mann-Whitney U, $\mathrm{p}<0.050$ for all comparisons; Fig. 5.1Bi). Symbiodinium C3 in I. palifera showed $\mathrm{F}_{\mathrm{v}} / \mathrm{F}_{\mathrm{m}}$ values in the control that were higher than at $28.5{ }^{\circ} \mathrm{C}$ at Day 6 (Mann-Whitney $\mathrm{U}$, $\mathrm{Z}=-1.991, \mathrm{p}<0.05$ ) and higher than at $31{ }^{\circ} \mathrm{C}$ from Days 1-6 (Mann-Whitney $\mathrm{U}$, $Z=-2.611, p<0.01$ for all comparisons; Fig. 5.1Ci). At all other time points, there was no difference between the control and the treatments in this host-symbiont combination (Mann-Whitney U, p < 0.05 for all comparisons). Symbiodinium type C15 in M. digitata displayed $\mathrm{F}_{\mathrm{v}} / \mathrm{F}_{\mathrm{m}}$ values in the control that were higher than at $28.5^{\circ} \mathrm{C}$ on Day 2 (MannWhitney $\mathrm{U}, \mathrm{Z}=-2.193, \mathrm{p}<0.03$ ) and $8 \%$ higher than at $31{ }^{\circ} \mathrm{C}$ at Day 6 (Mann-Whitney $\mathrm{U}$, $\mathrm{Z}=-1.984, \mathrm{p}<0.05$; Fig. 5.1Di). $\mathrm{F}_{\mathrm{v}} / \mathrm{F}_{\mathrm{m}}$ of Symbiodinium $\mathrm{C} 15$ in $P$. cylindrica did not show a change between the control and the $28.5^{\circ} \mathrm{C}$ treatments, but was $22-64 \%$ higher in the control than in the $31{ }^{\circ} \mathrm{C}$ treatment on all days (Mann-Whitney $\mathrm{U}, \mathrm{p}<0.03$ for all comparisons; Fig. 5.1Ei).

When comparing results for the same Symbiodinium types inside different coral species, $\mathrm{C} 3$ in I. palifera showed higher $\mathrm{F}_{\mathrm{v}} / \mathrm{F}_{\mathrm{m}}$ values than $\mathrm{C} 3$ in A. millepora at $31{ }^{\circ} \mathrm{C}$ at Day 6 (Mann-Whitney $\mathrm{U}, \mathrm{Z}=-2.353, \mathrm{p}<0.02$ ). $\mathrm{F}_{\mathrm{v}} / \mathrm{F}_{\mathrm{m}}$ of $\mathrm{C} 15$ in $M$. digitata was higher than in P. cylindrica at $31{ }^{\circ} \mathrm{C}$ from Day 2 until the end of the experiment (Mann-Whitney U, $\mathrm{p}<0.030$ for all comparisons). When comparing different Symbiodinium types, at $31{ }^{\circ} \mathrm{C}$, $\mathrm{F}_{\mathrm{v}} / \mathrm{F}_{\mathrm{m}}$ of $\mathrm{C} 15$ in $M$. digitata was higher than for $\mathrm{C} 3$ in both coral species (I. palifera and A. millepora) from Day 3 until the end of the experiment (Mann-Whitney $\mathrm{U}, \mathrm{p}<0.01$ for all comparisons). Also at $31{ }^{\circ} \mathrm{C}$, type $\mathrm{C} 1$ displayed $\mathrm{F}_{\mathrm{v}} / \mathrm{F}_{\mathrm{m}}$ values after one day which were lower than of type C3 in I. palifera and type C15 in P. cylindrica (Mann-Whitney U, $\mathrm{p} \leq 0.005$ for both comparisons). 
Chapter 5
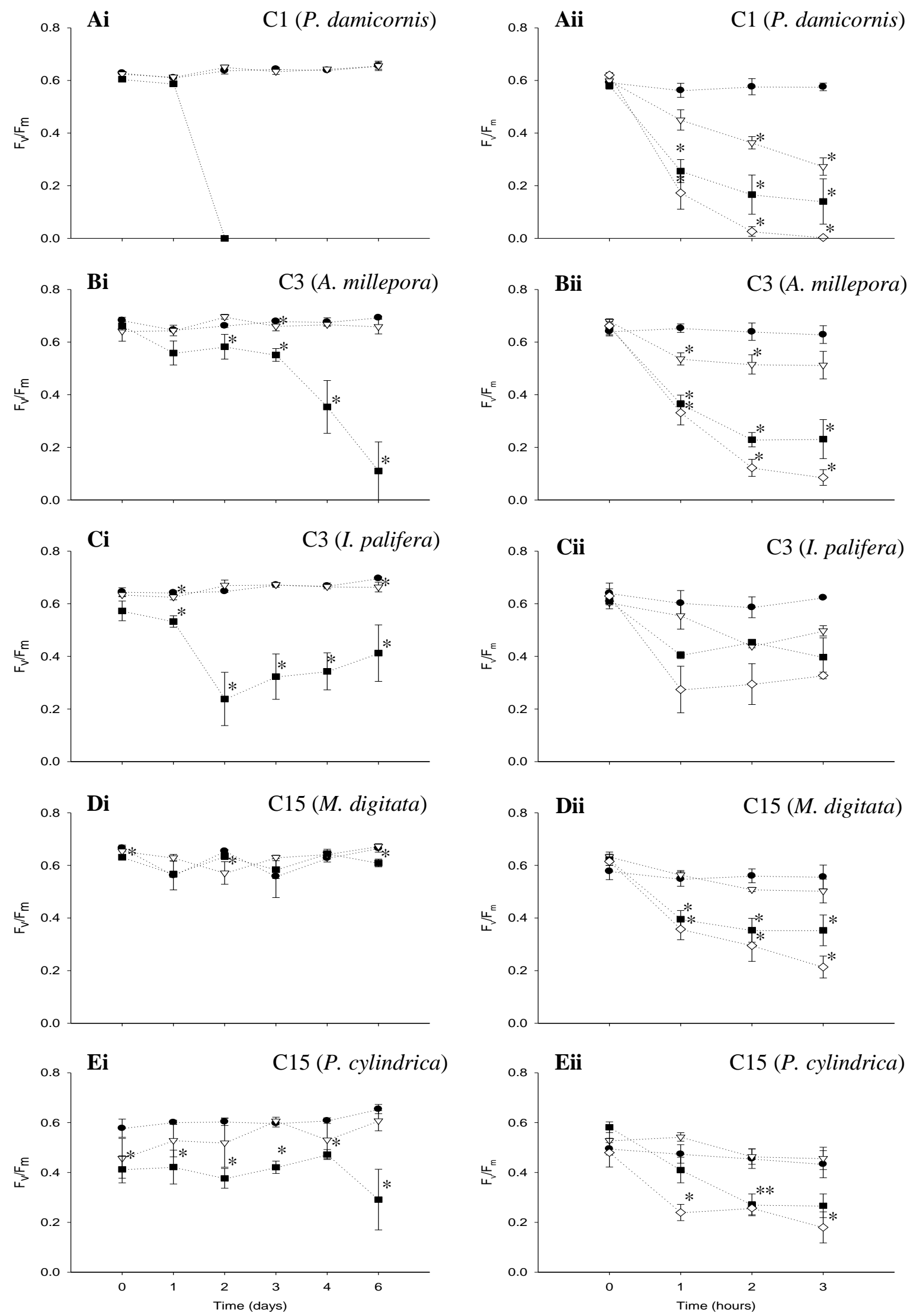

Figure 5.1: Maximum quantum yield $\left(\mathrm{F}_{\mathrm{v}} / \mathrm{F}_{\mathrm{m}}\right)$ of three Symbiodinium types in five different coral species at different temperatures (Ai-Ei; $\bullet: 26^{\circ} \mathrm{C} ; \nabla: 28.5^{\circ} \mathrm{C} ; \mathbf{m :} 31^{\circ} \mathrm{C}$ ) and hydrogen peroxide 
$\left(\mathrm{H}_{2} \mathrm{O}_{2}\right)$ concentrations (Aii-Eii; $\bullet: 0 \mathrm{mM}: \nabla 10 \mathrm{mM}$; $\mathbf{m}: 20 \mathrm{mM} ; \diamond: 40 \mathrm{mM}$ ) over time. Means $\pm \mathrm{SE}$ $(\mathrm{n}=5$ per time point); significant differences $(\mathrm{p}<0.05)$ between the control and treatment at the respective time point are represented with *. Note: dotted line used despite these being discrete samples, to clarify trends.

There were no differences in Symbiodinium cell density between any coral species at the beginning of the experiment (Tukey HSD, $\mathrm{p}>0.05$ ), but a time effect was observed in the different species (rANOVA with Greenhouse-Geisser correction, $F_{(7.363)}=3.842$, $\mathrm{p} \leq$ 0.001; Fig. 5.2). After six days of exposure to the different temperatures, Symbiodinium density was lower at $31^{\circ} \mathrm{C}$ than in the control explants for type $\mathrm{C} 3$ in A. millepora (Tukey HSD, $\mathrm{p}<0.03)$. At the same time point, C3 in I. palifera and C15 in P. cylindrica displayed lower cell densities at $31^{\circ} \mathrm{C}$ than at 26 and $28.5^{\circ} \mathrm{C}$ (Tukey HSD, $\mathrm{p} \leq 0.03$ ). There was no difference in cell density between any of the treatments for C15 in M. digitata throughout the experiment (Tukey HSD, p > 0.05). Symbiodinium cell density was correlated with chlorophyll $a$ concentration in both I. palifera and P. cylindrica (Pearson $\mathrm{r}=0.371, \mathrm{p}<0.001$ for overall correlation; Fig. 5.2A) and chlorophyll $\mathrm{c}_{2}$ in all coral species except for P. damicornis (Pearson $\mathrm{r}=0.588, \mathrm{p}<0.001$; Fig. 5.2B).
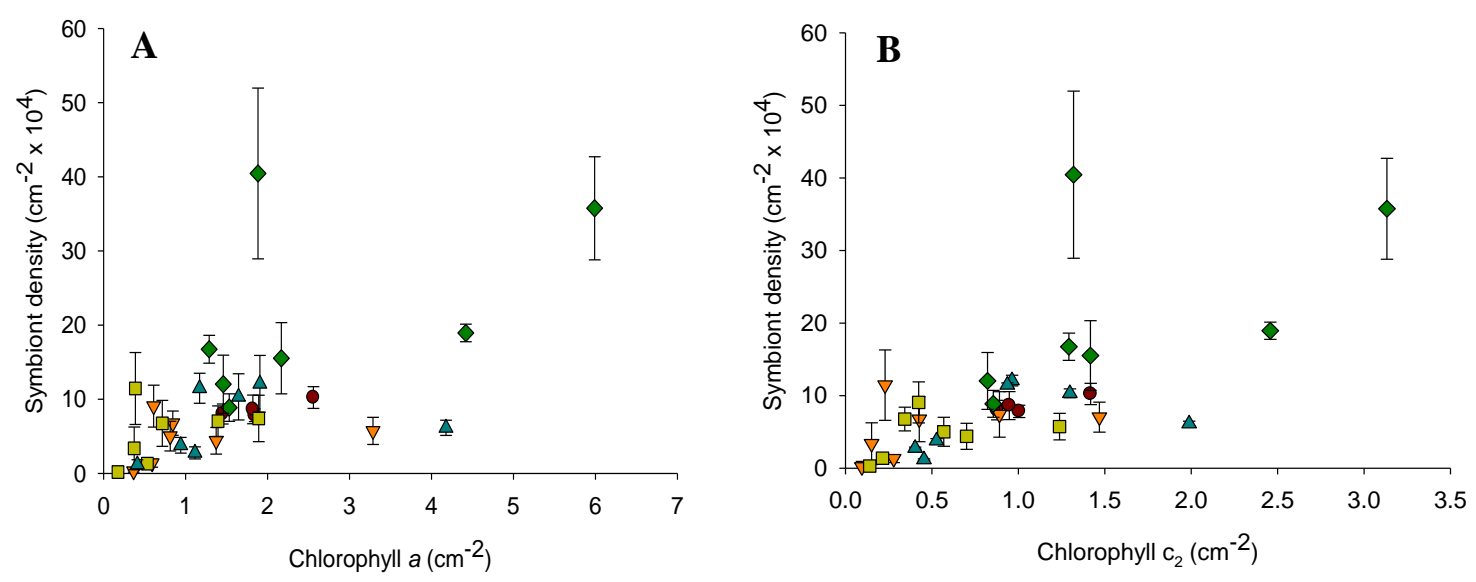

Figure 5.2: Symbiodinium cell numbers and corresponding chlorophyll $a$ (A) and $c_{2}$ (B) concentration per coral host surface area for three Symbiodinium types in five coral species (red: C1 (P. damicornis); orange: C3 (A. millepora); yellow: C3 (I. palifera); green: C15 (M. digitata); blue: C15 ( $P$. cylindrica). Data from Day 1, 2 and 6 when cells were exposed to 26, 28.5 and $31{ }^{\circ} \mathrm{C}$. Values are means $\pm \mathrm{SE}(\mathrm{n}=5)$ 
When subjecting Symbiodinium cells in hospite to increasing oxidative stress induced by hydrogen peroxide $\left(\mathrm{H}_{2} \mathrm{O}_{2} ; 10,20\right.$ and $\left.40 \mathrm{mM}\right)$, a time and $\mathrm{H}_{2} \mathrm{O}_{2}$ concentration effect on the maximum yield $\left(\mathrm{F}_{\mathrm{v}} / \mathrm{F}_{\mathrm{m}}\right)$ was observed (rANOVA with Greenhouse-Geisser correction, $\left.\mathrm{F}_{(7.744)}=23.731, \mathrm{p}<0.001\right)$. After just one hour of exposure to $40 \mathrm{mM} \mathrm{H}_{2} \mathrm{O}_{2}, \mathrm{~F}_{\mathrm{v}} / \mathrm{F}_{\mathrm{m}}$ in all treatments was lower than in the corresponding controls, and it remained significantly lower throughout the experiment (Tukey HSD, $\mathrm{p}<0.005$ ). However, the responses to the other $\mathrm{H}_{2} \mathrm{O}_{2}$ concentrations were different between types. After three hours of exposure, $\mathrm{F}_{\mathrm{v}} / \mathrm{F}_{\mathrm{m}}$ in the control of Symbiodinium $\mathrm{C} 1$ in P. damicornis was higher than at both 10 and $20 \mathrm{mM} \mathrm{H}_{2} \mathrm{O}_{2}$ (Tukey HSD, $\mathrm{p} \leq 0.001$ in all comparisons; Fig. 5.1Aii), while for types $\mathrm{C} 3$ in A. millepora and C15 in M. digitata $\mathrm{F}_{\mathrm{v}} / \mathrm{F}_{\mathrm{m}}$ in the control was higher than at $20 \mathrm{mM} \mathrm{H}_{2} \mathrm{O}_{2}$ (Tukey HSD, p < 0.05 for all comparisons; Fig. 5.1Bii, 5.1Dii). In comparison, for type $\mathrm{C} 15$ in $P$. cylindrica at this same time point, a decline in $\mathrm{F}_{\mathrm{v}} / \mathrm{F}_{\mathrm{m}}$ was only apparent at $40 \mathrm{mM} \mathrm{H}_{2} \mathrm{O}_{2}$ (Tukey HSD, $\mathrm{p}<0.02$; Fig. 5.1Eii), however statistical comparison of type C15 in the two coral species did not reveal any significant differences between them in any treatment at any sampling point (Mann-Whitney $\mathrm{U}, \mathrm{p}>0.05$ ).

Different responses to oxidative stress were also apparent when the different symbiont types were compared. $\mathrm{F}_{\mathrm{v}} / \mathrm{F}_{\mathrm{m}}$ of type $\mathrm{C} 1$ was lower than for $\mathrm{C} 15$ in both host species after two and three hours of exposure to $40 \mathrm{mM} \mathrm{H}_{2} \mathrm{O}_{2}$ (Tukey HSD, p < 0.005). Similarly, type C3 in A. millepora was more affected than C15 in either host species after two hours at $40 \mathrm{mM} \mathrm{H} \mathrm{O}_{2}$ (Tukey HSD, $\mathrm{p}<0.050$ for both comparisons).

No changes in Symbiodinium cell density or chlorophyll $a$ and $\mathrm{c}_{2}$ concentrations were observed during the three hour exposure to oxidative stress (two-way ANOVA, $\mathrm{p}>0.05$ ).

\subsubsection{Photosynthetic parameters for freshly isolated Symbiodinium}

The effects of thermal and oxidative stress were tested on two types of freshly isolated Symbiodinium. After a 60-min exposure to three temperatures, the isolated C3 type from I. palifera displayed $\mathrm{F}_{\mathrm{v}} / \mathrm{F}_{\mathrm{m}}$ values that, at 28.5 and $31{ }^{\circ} \mathrm{C}$, were 71 and $85 \%$ of the control values, respectively (Mann-Whitney U, p $<0.01$ for both comparisons; Fig. 5.3Ai). The 

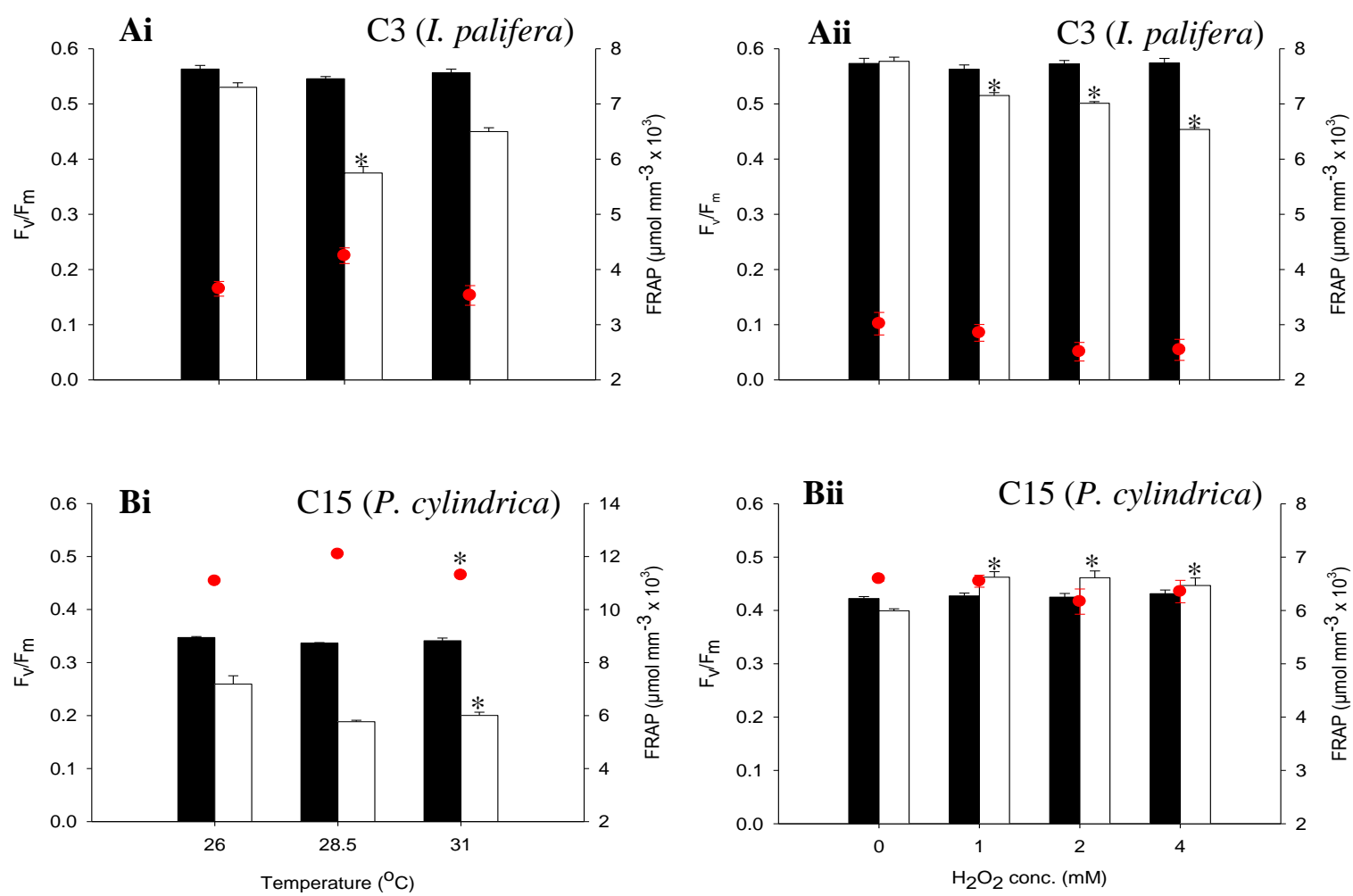

Figure 5.3: Maximum quantum yield $\left(\mathrm{F}_{\mathrm{v}} / \mathrm{F}_{\mathrm{m}}\right.$; bars) and total antioxidant potential (FRAP; red dots) in two types of freshly isolated Symbiodinium (A: C3 from I. palifera; B: C15 from P. cylindrica) at different temperatures $(\mathrm{Ai}, \mathrm{Bi})$ and different concentrations of hydrogen peroxide $\left(\mathrm{H}_{2} \mathrm{O}_{2}\right.$; Aii, Bii) over time (black bars: time 0 ; white bars: 60 min exposure). Values are means $\pm S E(n=4)$; significant differences between the control and treatment are represented as *. Please note: FRAP axis in Bi differs from the rest.

isolated $\mathrm{C} 15$ type from $P$. cylindrica showed $\mathrm{F}_{\mathrm{v}} / \mathrm{F}_{\mathrm{m}}$ values in the $31{ }^{\circ} \mathrm{C}$ treatment that were $22 \%$ lower after 60 min than in the control (Mann-Whitney U, p $\leq 0.025$; Fig. 5.3Bi).

$\mathrm{F}_{\mathrm{v}} / \mathrm{F}_{\mathrm{m}}$ changed after 60 min of exposure to various $\mathrm{H}_{2} \mathrm{O}_{2}$ concentrations $(1,2$ and $4 \mathrm{mM})$, in the case of both Symbiodinium types (rANOVA, Greenhouse-Geisser, $\mathrm{F}_{(6)}=39.207$, $\mathrm{p}<0.001) . \mathrm{F}_{\mathrm{v}} / \mathrm{F}_{\mathrm{m}}$ for type $\mathrm{C} 3$ in the control was higher than in all other treatments and was $21 \%$ higher than in the $4 \mathrm{mM} \mathrm{H}_{2} \mathrm{O}_{2}$ treatment (Tukey HSD, $\mathrm{p}<0.001$; Fig. 5.3Aii). $\mathrm{F}_{\mathrm{v}} / \mathrm{F}_{\mathrm{m}}$ in the $4 \mathrm{mM} \mathrm{H}_{2} \mathrm{O}_{2}$ treatment was also lower than in the 1 and $2 \mathrm{mM} \mathrm{H}_{2} \mathrm{O}_{2}$ treatments (Tukey HSD, $\mathrm{p}<0.001$ for both comparisons). In comparison, $\mathrm{F}_{\mathrm{v}} / \mathrm{F}_{\mathrm{m}}$ for type $\mathrm{C} 15$ was different between the control and $\mathrm{H}_{2} \mathrm{O}_{2}$ treatments, and in fact increased by $12 \%$ during the 


\section{Chapter 5}

one-hour exposure to $4 \mathrm{mM} \mathrm{H}_{2} \mathrm{O}_{2}$ (Tukey $\mathrm{HSD}$, p $<0.001$ for all comparisons; Fig. 5.3Bii).

\subsubsection{Antioxidant capacity in Symbiodinium cells in hospite}

SOD and APX activity were measured in Symbiodinium cells in hospite at 0,2 and 6 days of the thermal stress experiment (Fig. 5.4Ai-Ei). The only change in SOD activity was in type C15 in $M$. digitata, which showed a 55\% lower SOD activity in the control than at $28.5^{\circ} \mathrm{C}$ after two days of exposure to the different temperatures (Tukey HSD, p < 0.050). There were no changes in APX activity at any of the three sampling points (Tukey HSD, $\mathrm{p}>0.050$ for all comparisons; Fig. 5.4Aii-Eii).

Antioxidant activity was also measured after four hours exposure to $\mathrm{H}_{2} \mathrm{O}_{2}$ (Fig. 5.5). In type $\mathrm{C} 15$ in $M$. digitata, SOD activity was $158 \%$ higher in the $40 \mathrm{mM} \mathrm{H}_{2} \mathrm{O}_{2}$ treatment than at $10 \mathrm{mM} \mathrm{H} \mathrm{H}_{2}$ (Tukey HSD, $\mathrm{p} \leq 0.025$ ). Similarly, SOD activity increased in $\mathrm{C} 15$ in P. cylindrica between the control and the 20 and $40 \mathrm{mM} \mathrm{H}_{2} \mathrm{O}_{2}$ treatments, by 103 and $114 \%$ respectively (Tukey HSD, $\mathrm{p}<0.015$ for both comparisons); moreover, SOD activity was 290 and $310 \%$ higher at 20 and $40 \mathrm{mM} \mathrm{H}_{2} \mathrm{O}_{2}$ than at $10 \mathrm{mM} \mathrm{H} \mathrm{O}_{2}$ (Tukey HSD, $\mathrm{p}<0.001)$. No other Symbiodinium type displayed any differences between treatments (Tukey HSD, p > 0.05).

APX activity at $40 \mathrm{mM} \mathrm{H} \mathrm{H}_{2} \mathrm{O}_{2}$ was higher than in all other treatments in all Symbiodinium types in hospite (Tukey HSD, $\mathrm{p}<0.05$ for all comparisons; Fig. 5.5B). This increase was considerable, ranging between $1800 \%$ for type $\mathrm{C} 1$ and $3250 \%$ for $\mathrm{C} 15$ in M. digitata relative to the control. No other differences between treatments were observed in any of the Symbiodinium types (Tukey HSD, $\mathrm{p}>0.05$ ). 

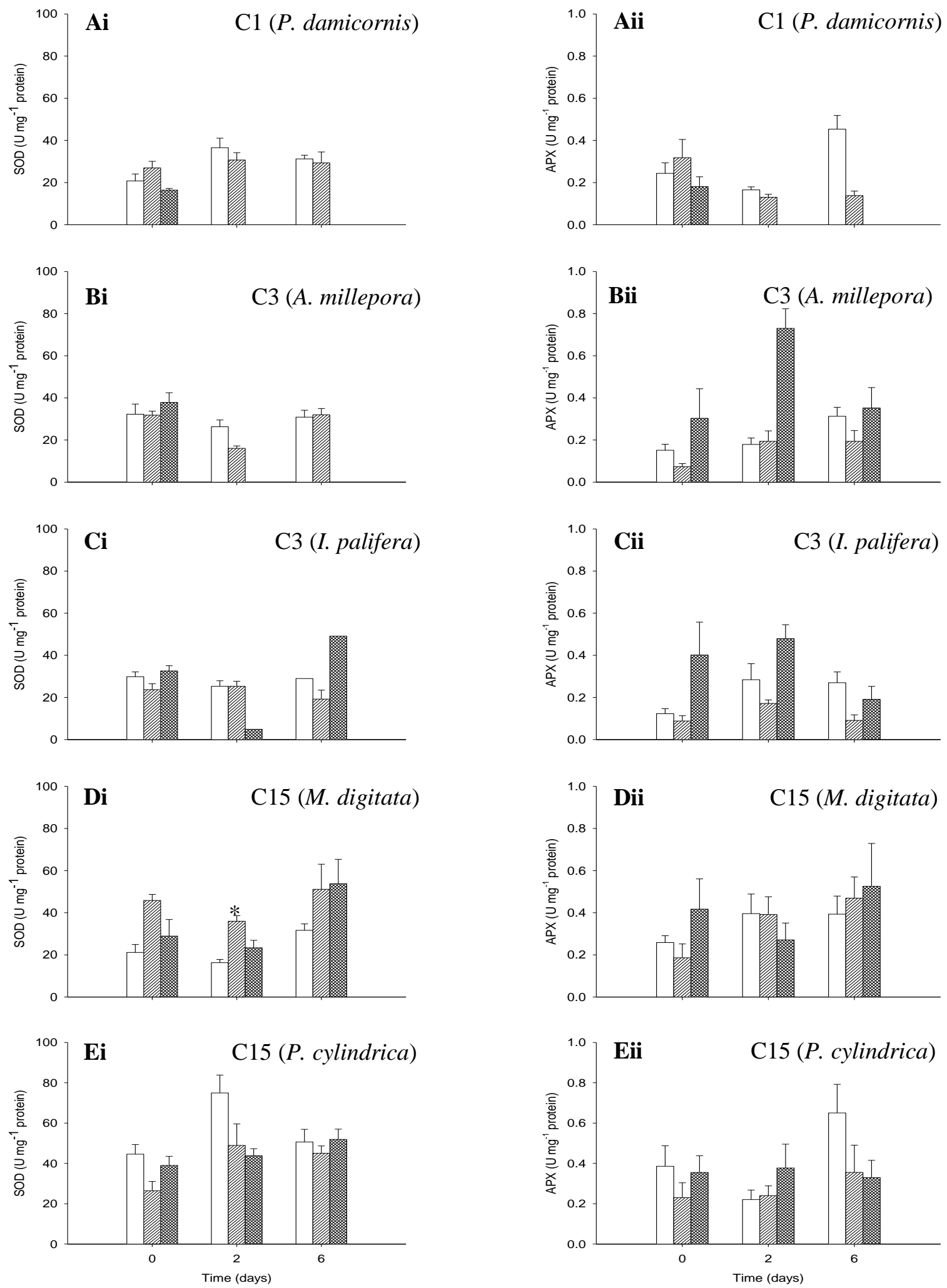

Figure 5.4: Superoxide dismutase (SOD; Ai-Ei) and ascorbate peroxidase (APX; Aii-Eii) activity ( $\mathrm{U} \mathrm{mg}^{-1}$ algal protein) for three Symbiodinium types in five different coral hosts at different temperatures ( $\square: 26{ }^{\circ} \mathrm{C}$; שा : $28.5{ }^{\circ} \mathrm{C}$; $31{ }^{\circ} \mathrm{C}$ ) before and after two and six days of exposure. Means $\pm \mathrm{SE}(\mathrm{n}=5$ per time point); significant differences $(\mathrm{p}<0.05)$ between the control and treatment at the respective time point are represented with *. 

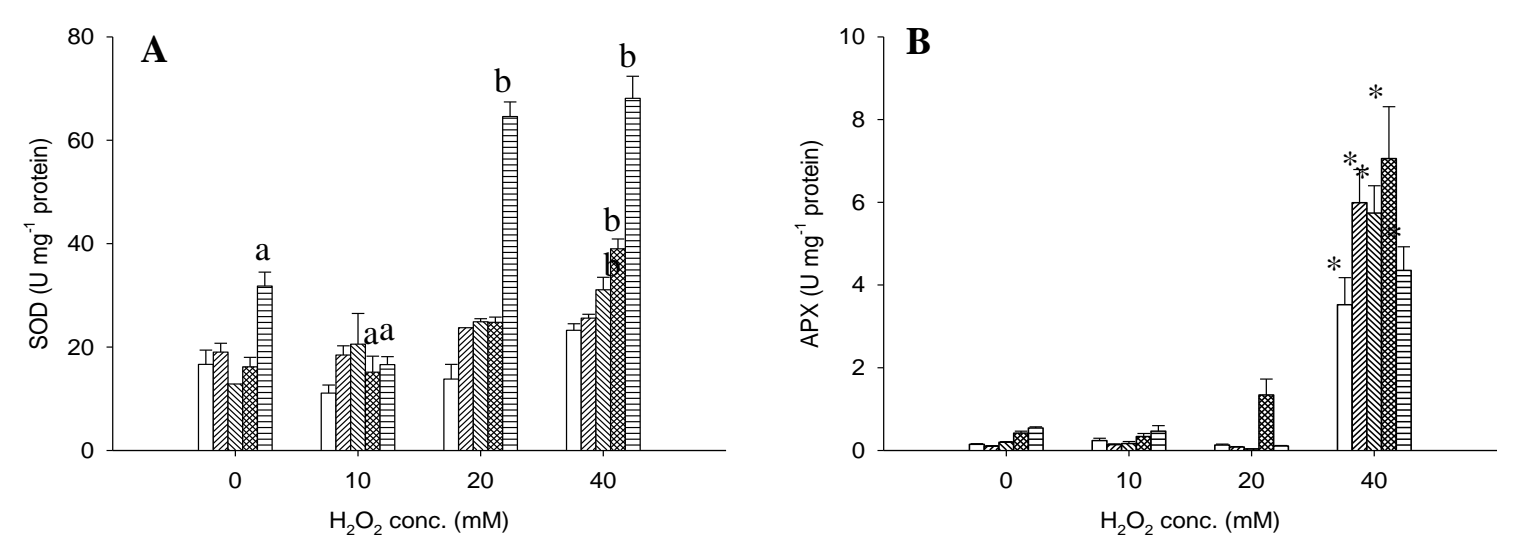

Figure 5.5: Superoxide dismutase (SOD; A) and ascorbate peroxidase (APX; B) activity ( $\mathrm{U} \mathrm{mg}^{-1}$ algal protein) at different hydrogen peroxide $\left(\mathrm{H}_{2} \mathrm{O}_{2}\right)$ concentrations $(0,10,20,40 \mathrm{mM})$ in three different Symbiodinium types in hospite in five coral species ( $\square$ : $\mathrm{C} 1$ (P. damicornis); שाय : C3 (A. millepora); : C3 (I. palifera); : C15 (M. digitata); $\rightleftharpoons: \mathrm{C} 15$ (P. cylindrica). Values are means $\pm \operatorname{SE}(n=5)$. Significant differences $(\mathrm{p} \leq 0.05)$ highlighted by letters $(a)$ and $(b)$; * shows significant differences between the $40 \mathrm{mM} \mathrm{H}_{2} \mathrm{O}_{2}$ treatment and all other treatments.

\subsubsection{Antioxidant capacity (FRAP) in freshly isolated Symbiodinium}

Total antioxidant potential was measured in the isolated Symbiodinium cells at the end of the thermal and oxidative stress experiments, via the FRAP assay. The assay detected an increase in antioxidant activity between the control and the $31{ }^{\circ} \mathrm{C}$ treatments in type $\mathrm{C} 15$ isolated from P. cylindrica (Mann-Whitney $\mathrm{U}, \mathrm{Z}=-2.249, \mathrm{p}<0.025$; Fig. 5.3Bii). There was no difference in FRAP values for type C3 from I. palifera (Mann-Whitney U, $\mathrm{p}>0.05)$. When the freshly isolated Symbiodinium cells were subjected to different $\mathrm{H}_{2} \mathrm{O}_{2}$ concentrations, no differences were apparent between the treatments in either type (Tukey HSD, p > 0.05; Fig. 5.3Ai, Bi).

\subsection{Discussion}

In this study, I demonstrated the effects of thermal and oxidative stress on photosynthetic efficiency and antioxidant defenses in different Symbiodinium types, when both in hospite and freshly isolated from corals. Marked differences were found between some of the 
Symbiodinium types, indicating differences in stress susceptibility, and the more thermally sensitive Symbiodinium types were also susceptible to oxidative stress. However, the typespecific response was influenced by the species of host coral. In particular, differences in stress response between Symbiodinium types were more marked when they were outside the host.

Thermal stress can impair the alga's photosynthetic apparatus, producing various reactive oxygen species (ROS) in the process (Venn et al. 2008). ROS like $\mathrm{H}_{2} \mathrm{O}_{2}, \mathrm{O}_{2}{ }^{-}$and ${ }^{1} \mathrm{O}_{2}$ can damage cell organelles and molecules causing intracellular oxidative stress. In both thermal and oxidative stress experiments in the current study, Symbiodinium type C1 in the coral P.damicornis was very susceptible to increasing stress. Indeed, there was no measurable chlorophyll fluorescence in hospite at $31{ }^{\circ} \mathrm{C}$ after two days of exposure. The highest $\mathrm{H}_{2} \mathrm{O}_{2}$ treatment had the same effect on the algal cell's photosystem after just three hours; this was the strongest response to oxidative stress observed. Type $\mathrm{C} 1$ has been shown to display variable thermal tolerances in the past, with host species seemingly having an impact. While Symbiodinium C1 was very tolerant to long-term high temperatures in A.tenuis (Abrego et al. 2008, Littman et al. 2010), its photosynthetic capacity decreased rapidly during short-term thermal stress while inside Stylophora pistillata (Fitt et al. 2009); this latter response was more comparable to that observed in the current study. Type C3 in A. millepora and I. palifera was more tolerant to both thermal and oxidative stress. During thermal stress, $\mathrm{F}_{\mathrm{v}} / \mathrm{F}_{\mathrm{m}}$ in both corals declined at $31{ }^{\circ} \mathrm{C}$ relative to the control from very early in the experiment. The magnitude of this decrease was the same in both coral species, becoming different only on the last day of exposure. In contrast, type $\mathrm{C} 15$ responded quite differently in its two coral hosts, with $\mathrm{F}_{\mathrm{v}} / \mathrm{F}_{\mathrm{m}}$ in $M$. digitata being higher than in $P$. cylindrica almost throughout the entire experimental period. This differential response has been observed before for type $\mathrm{C} 15$, which was more susceptible to a slow increase in temperature in Porites lutea than in Montipora digitata (Fisher 2006). In the current study, C15 in M. digitata also showed a higher tolerance to increasing temperature and $\mathrm{H}_{2} \mathrm{O}_{2}$, being the most resistant type. Its photosynthetic capacity was often higher than for Symbiodinium C1 and C3 under both stress conditions, as has also been shown previously (Fitt et al. 2009, Fisher et al. 2012, Wang et al. 2012). 


\section{Chapter 5}

Symbiodinium C15 from P. cylindrica was less tolerant to increased temperature and showed a more similar response to $\mathrm{C} 3$.

Symbiodinium types C3 and C15 in P. cylindrica displayed decreases in cell density at higher temperatures by the end of the exposure time, suggesting the onset of bleaching. While a decrease in $\mathrm{F}_{\mathrm{v}} / \mathrm{F}_{\mathrm{m}}$ does not necessarily predict coral bleaching (Fisher et al. 2012), both factors indicate a decline in the general health of the holobiont. Occurrence of bleaching in both coral species containing Symbiodinium C3, and in P. cylindrica containing type $\mathrm{C} 15$ underlines their greater stress susceptibility in comparison to M. digitata with type C15. In comparison, the two species that contained C15 types were equally tolerant to increased $\mathrm{H}_{2} \mathrm{O}_{2}$. Overall, it appears that Symbiodinium C1 was the most susceptible type to stress, while types C3 and C15 are more similar in their responses. Differences between these two Symbiodinium types appear to depend mainly on the host species, which of course could also play a role in the sensitivity of type $\mathrm{C} 1$ seen here.

When inside their animal hosts, Symbiodinium cells can be protected in several ways by the coral. For instance, both the coral and symbiont can synthesize heat shock proteins (Hsps). These proteins can lead to a higher thermotolerance in the symbiotic partners (Black et al. 1995, Leggat et al. 2007). A number of different Hsps are found in coral tissue and areas of higher concentrations appear to bleach less (Baird et al. 2009). Most corals also contain a family of proteins called fluorescent pigments (FPs) that are closely related to the green fluorescent proteins (Baird et al. 2009). These FPs play a photoprotective role and reduce photoinhibition by absorbing, scattering and dissipating high-energy solar radiation via fluorescence (Salih et al. 2000). FPs also have very high $\mathrm{H}_{2} \mathrm{O}_{2}$ scavenging activities (Palmer et al. 2009, 2010) which might have played an important protective role in the oxidative stress experiment. It also appears that more bleaching susceptible coral species contain lower densities of FPs (Baird et al. 2009). This might have been the case in P. damicornis, which displayed a low tolerance to $\mathrm{H}_{2} \mathrm{O}_{2}$, and has been shown to contain less FPs than the other coral species used in the current study (Palmer et al. 2010). Under stress conditions, mycosporine-like amino acids (MAAs) can absorb ultraviolet (UV) radiation and dissipate UV energy (Shick and Dunlap 2002, Shick 2004). The abundance and diversity of MAAs is much higher in the holobiont and host 
tissue than in freshly isolated Symbiodinium (Shick and Dunlap 2002). Therefore, it appears that the host influences the diversity and distribution of MAAs, and subsequently the amount of damage sustained by the symbiont, as well as possibly producing MAAs itself (Ferrier-Pagés et al. 2007, Baird et al. 2009, Shinzato et al. 2011). By building the first line of defense against harmful UV, MAAs act as a natural sunscreen in the coraldinoflagellate symbiosis.

UV- or light-stress in the symbiont is also affected by the growth form of the coral. Coral tissue thickness is especially important as it might provide protection through self-shading of the symbionts within (Hoegh-Guldberg 1999, Loya et al. 2001, Hughes et al. 2012). A. millepora, I. palifera, P. cylindrica and M. digitata all have similar growth forms with thick, robust branches (Kelley 2009). In M. digitata, which has a relatively high symbiont cell density, self-shading probably contributed as a protective mechanism, as previously observed by Fisher (2006). P. damicornis on the other hand has very many fine branches with much thinner host tissue (Loya et al. 2001).

Most likely due to these various host-related factors, differences in the stress responses were seen in the current study between the same Symbiodinium type but when associated with different coral species. To resolve the host's impact on these differences, the effects of thermal and oxidative stress on Symbiodinium cells that were freshly isolated from the same coral species were tested. Unfortunately, a number of the cell isolates were not photosynthetically viable, as has been shown in the past (Wang et al. 2011); however, Symbiodinium C3 from I. palifera and C15 from P. cylindrica were successfully isolated. Type C3, which showed a similar stress response to type C15 when in hospite, displayed a much lower photosynthetic capacity under oxidative stress when in the isolated state. Similarly, Fisher (2006) noted that stress responses between Symbiodinium types in different coral species tend to be more similar in the symbiotic than in the freshly-isolated state. This might indicate that type C3 in I. palifera is more reliant on its host's protective mechanisms than is $\mathrm{C} 15$ in $P$. cylindrica. 
Symbiodinium cells also have their own means to protect themselves from the effects of thermal and oxidative stress. Algae can use non-photochemical quenching (NPQ) to dissipate excess photosynthetic energy as heat and prevent it from damaging the photosynthetic apparatus (Müller et al. 2001, Hill et al. 2005). NPQ helps to avoid a buildup of energy and stress occurring in the cells. However, if NPQ is overwhelmed, a subsequent production of ROS can often be countered by various antioxidants (Lesser 2006). Examples for non-enzymatic antioxidants include carotenoids, which play a role in NPQ, and glutathione, which reacts with ${ }^{1} \mathrm{O}_{2}, \mathrm{O}_{2}{ }^{-}$and the $\mathrm{OH}^{*}$. However, in the first line of defense against ROS are enzymatic antioxidants such as SOD, which reduces $\mathrm{O}_{2}{ }^{-}$to $\mathrm{H}_{2} \mathrm{O}_{2}$, and APX and CAT, which reduce $\mathrm{H}_{2} \mathrm{O}_{2}$ to water and oxygen (Venn et al. 2008). When SOD and APX were measured at several time points during the six-day period of the thermal stress experiment, no changes were apparent in the Symbiodinium cells. One explanation might have been that the temperature stress was not strong enough to induce an antioxidant response in the algal cells, but as photosynthetic efficiency and other parameters declined over the course of exposure, this explanation is not very likely. Also, Higuchi et al. (2008) found an increase in SOD and CAT in Symbiodinium cells in hospite at $31{ }^{\circ} \mathrm{C}$, the same temperature as used in my study. More probably, antioxidants were indeed produced in the Symbiodinium cells but were readily used to remove any generated ROS. These antioxidants might have prevented detrimental effects from being apparent at $29{ }^{\circ} \mathrm{C}$. Indeed, turnover rates might have been amplified by the increase in temperature (Eckert and Randall 1978, Putnam and Edmunds 2010). Further experimentation, with more time points than were used here, will help to resolve the pattern of events.

In comparison, APX production was heavily up-regulated in all Symbiodinium types at the highest $\mathrm{H}_{2} \mathrm{O}_{2}$ concentration. As this antioxidant reduces $\mathrm{H}_{2} \mathrm{O}_{2}$, its production is the first step to counteract the increasing stress (Alscher et al. 1997, Asada 2000). In contrast, no changes in APX activity were observed at the other $\mathrm{H}_{2} \mathrm{O}_{2}$ concentrations. This might indicate that the $\mathrm{H}_{2} \mathrm{O}_{2}$ concentrations were either not high enough to cause an immediate defense response within the short time frame of the experiment, or that any up-regulated APX was immediately used to remove $\mathrm{H}_{2} \mathrm{O}_{2}$. SOD was up-regulated in type $\mathrm{C} 15$, consistent with its greater oxidative-stress tolerance. However, no SOD generation was observed in the other two Symbiodinium types. It is possible that the added $\mathrm{H}_{2} \mathrm{O}_{2}$ did not 
cause sufficient damage in the cell. More likely, though, is that there was no increase in SOD activity due to inhibition of this enzyme by its end product, $\mathrm{H}_{2} \mathrm{O}_{2}$ (Cheng and Song 2006).

When testing total antioxidant capacity in freshly isolated Symbiodinium cells under thermal and oxidative stress, not many treatment effects could be observed for each of the different types. This response was also observed in other stress experiments with freshly isolated Symbiodinium cells from sea anemones (see Appendix B). The reasons for this could include the exposure time being too short or incomplete separation of host tissue and symbiont cells during isolation. Also any animal or other cellular material present in the sample could have contaminated the assay, masking the Symbiodinium response. Even though no differences between treatments could be found, antioxidant activity (expressed as FRAP values) differed substantially between the two Symbiodinium types tested. Under both stressors, type C15 from $P$. cylindrica showed higher total antioxidant activity than type C3 from I. palifera. These high baseline antioxidant levels further corroborate the status of C15 as a very tolerant Symbiodinium type.

In this study, I tested the effects of elevated temperature and $\mathrm{H}_{2} \mathrm{O}_{2}$ concentration on Symbiodinium cells in different states of symbiosis. A host species influence on the Symbiodinium cells was detected in hospite, with the symbiont response in isolation being different. Furthermore, holobiont- as well as type-specific responses were observed during stress that can provide insight into how these specific Symbiodinium-cnidarian associations will behave in future bleaching scenarios. I have also shown how stress events may induce intracellular protective mechanisms like the production of antioxidants. This study provides evidence about the diversity of stress responses in different, closely related Symbiodinium types. At the same time, it underlines the importance of studying Symbiodinium in hospite, as part of the holobiont, for a realistic picture of the processes occurring on coral reefs. 


\section{Chapter 5}




\section{Chapter 6: General discussion}

In this thesis, the effects of thermal and oxidative stress induced by hydrogen peroxide $\left(\mathrm{H}_{2} \mathrm{O}_{2}\right)$ were tested on a range of Symbiodinium clades and types, in different states of symbiosis (in culture, freshly isolated, in hospite) and from different geographic origins. The aim was to explore the diversity of type-specific stress responses, and to investigate the relationship between thermal and oxidative stress, as well as possible protective mechanisms, that could influence the bleaching response of corals and anemone hosts.

\subsection{Diversity in stress response}

This work shows that different clades of Symbiodinium and even closely related types (i.e. sub-clades) display an immense variation in their response to thermal and oxidative stress. Generally, when examining the effects of stress on photosynthetic health of the dinoflagellate cells, a decrease in photosynthetic efficiency of PSII, as described by the maximum quantum yield of PSII $\left(\mathrm{F}_{\mathrm{v}} / \mathrm{F}_{\mathrm{m}}\right)$ and the maximum electron transport rate between photosystems $\left(\mathrm{ETR}_{\max }\right)$, was observed (Chapters 2, 5). At the same time, the production of different reactive oxygen species (ROS) such as hydrogen peroxide $\left(\mathrm{H}_{2} \mathrm{O}_{2}\right)$, superoxide $\left(\mathrm{O}_{2}{ }^{-}\right)$, and singlet oxygen $\left({ }^{1} \mathrm{O}_{2}\right)$ clearly increased inside most dinoflagellate cells when stressed by high temperature or $\mathrm{H}_{2} \mathrm{O}_{2}$ at different concentrations (Chapter 3; Table 6.1).

These data suggest that increased thermal and oxidative stress have a negative impact on the alga's photosynthetic apparatus. Thermal stress is an external factor which can impair PSII, probably via the D1 protein, as well as damage thylakoid membranes and cause a disruption of carboxylation by Rubisco in the Calvin-Benson cycle (Apel and Hirt 2004, Tchernov et al. 2004, Takahashi and Murata 2008, Venn et al. 2008). The impairment of photosystems can lead to photoinhibition and a build-up of excess energy; as a result, electrons are not transported correctly between photosystems. Instead, they are transferred onto oxygen molecules forming ${ }^{1} \mathrm{O}_{2}$ in PSII, and $\mathrm{O}_{2}{ }^{-}$via the Mehler reaction (Badger et al. 
2000, Tchernov et al. 2004), with the latter being reduced to $\mathrm{H}_{2} \mathrm{O}_{2}$ by the antioxidant superoxide dismutase (SOD; Veljović-Jovanović 1998, Asada 2000). When added to the algal cells to simulate oxidative stress, $\mathrm{H}_{2} \mathrm{O}_{2}$ quickly permeates the cell walls and chloroplasts (Downs et al. 2002). The presence of the pro-oxidant has an indirect but immediate effect on the amount of $\mathrm{O}_{2}{ }^{-}$inside the cells, because $\mathrm{H}_{2} \mathrm{O}_{2}$ inhibits SOD enzyme activity in a negative feedback reaction (Cheng and Song 2006). These processes do not necessitate any impairment of the photosynthetic apparatus. In comparison, the production of ${ }^{1} \mathrm{O}_{2}$ in response to the addition of $\mathrm{H}_{2} \mathrm{O}_{2}$ is likely to be a less direct effect, and indicates the involvement of PSII. Damage to the photosystem could have led to a formation of triplet chlorophyll in PSII, the starting point for ${ }^{1} \mathrm{O}_{2}$ production (Veljović-Jovanović 1998, Apel and Hirt 2004, Lesser 2006). Indeed, in the present study ${ }^{1} \mathrm{O}_{2}$ production only occurred under prolonged thermal and oxidative stress, and not in all Symbiodinium types (Chapter 3; Table 6.1).

All of these processes of ROS formation can take place inside algal cells during stress, but severity might vary between the different Symbiodinium clades and types, so explaining variations in the bleaching response. A range of mechanisms can prevent impairment of the photosystems and photoinhibition, and consequently prevent or slow down the generation of ROS. The D1 protein in PSII is one of the sites of impairment in the photosynthetic apparatus, where it plays an important structural and functional role (Warner et al. 1999, Lesser and Farrell 2004). While the D1 protein has a fast turnover with high re-synthesis and replacement rates, high temperature and light can inhibit the repair mechanisms that can lead to a loss of PSII activity (Takahashi and Murata 2008, Takahashi et al. 2009). Some Symbiodinium types might be more efficient in D1 repair than others, and therefore better able to counteract its impairment and the consequences of this (Takahashi et al. 2009, Hennige et al. 2011). A protective, rather than a repair mechanism of the photosystems is non-photochemical quenching (NPQ; Müller et al. 2001). During thermal and light stress, chlorophyll in its singlet excited state can be quenched to the ground state by internal conversion of xanthophyll pigments. Thus, excess excitation energy is harmlessly dissipated as heat instead of being used in photosynthesis (Gorbunov et al. 2001, Hill et al. 2005). Many studies have shown that different Symbiodinium types use NPQ to different extents to protect the photosystems from damage; this might explain 
Table 6.1: Simplified summary of results from Chapters 2-4 with different Symbiodinium types (in culture, freshly isolated) under thermal stress and hydrogen peroxide $\left(\mathrm{H}_{2} \mathrm{O}_{2}\right)$ induced oxidative stress. $\uparrow$ and $\downarrow$ arrows represent increase and decrease of measured parameters $\left(\mathrm{F}_{\mathrm{v}} / \mathrm{F}_{\mathrm{m}}\right.$, production of general ROS, superoxide $\mathrm{O}_{2}{ }^{-}$and singlet oxygen ${ }^{1} \mathrm{O}_{2}$, and antioxidant activity) respectively, while - represents no change.

\begin{tabular}{|c|c|c|c|c|c|c|c|c|c|c|}
\hline & \multicolumn{4}{|c|}{ Thermal stress } & \multicolumn{6}{|c|}{ Oxidative stress $\left(\mathrm{H}_{2} \mathrm{O}_{2}\right)$} \\
\hline & Chapter 2 & & Chapter 3 & & & $\operatorname{ter} 2$ & & Chapter 3 & & Chapter 4 \\
\hline Type & $F_{v} / F_{m}$ & ROS prod. & $\mathrm{O}_{2}$ prod. & ${ }^{\mathrm{T}} \mathrm{O}_{2}$ prod. & $\mathrm{F}_{\mathrm{v}} / \mathrm{F}_{\mathrm{m}}$ & $\mathbf{P} / \mathbf{R}$ & ROS prod. & $\mathrm{O}_{2}$ prod. & ${ }^{\mathrm{T}} \mathrm{O}_{2}$ prod. & $\begin{array}{l}\text { Antioxidant } \\
\text { activity }\end{array}$ \\
\hline A1 & $\downarrow \downarrow \downarrow$ & $\uparrow \uparrow \uparrow$ & $\uparrow \uparrow \uparrow$ & - & - & - & $\uparrow \uparrow \uparrow$ & - & - & $\uparrow$ \\
\hline B2 & $\downarrow$ & $\uparrow \uparrow \uparrow$ & $\uparrow \uparrow$ & - & $\downarrow \downarrow \downarrow$ & $\downarrow \downarrow \downarrow$ & $\uparrow \uparrow \uparrow$ & $\uparrow \uparrow$ & - & \\
\hline E1-0 & $\downarrow \downarrow$ & $\uparrow$ & - & - & $\downarrow \downarrow$ & $\uparrow$ & $\downarrow \downarrow$ & - & 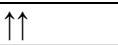 & $\downarrow$ \\
\hline F1-H & $\uparrow$ & $\uparrow$ & - & - & $\downarrow \downarrow \downarrow$ & $\downarrow \downarrow \downarrow$ & - & $\uparrow \uparrow$ & 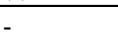 & $\uparrow$ \\
\hline F1-P & $\downarrow \downarrow$ & & & & $\downarrow \downarrow$ & $\downarrow$ & & & & \\
\hline S. californium & $\downarrow \downarrow$ & $\uparrow$ & - & - & $\downarrow \downarrow$ & $\downarrow$ & $\uparrow \uparrow \uparrow$ & $\uparrow \uparrow \uparrow$ & $\uparrow \uparrow$ & \\
\hline A1-FIS & - & & & & - & $\uparrow$ & & & & \\
\hline B1-FIS & - & & & & $\downarrow \downarrow$ & $\downarrow \downarrow$ & & & & \\
\hline
\end{tabular}

varying stress susceptibilities (Ragni et al. 2010, Hennige et al. 2011). A similar role is played by mycosporine-like amino acids (MAAs). These act as natural sunscreens for the coral-algal symbiosis by absorbing UV radiation and dissipating UV energy (Shick 2004). MAAs act as a first line of defence against harmful UV rays and protect the photosynthetic apparatus from damage (Shick and Dunlap 2002). A range of different MAAs exists and again, quality and quantity of synthesis might vary between Symbiodinium types and influence their ability to handle conditions of high UVR. In coral hosts, fluorescent proteins (FPs) can be produced which dissipate high-energy solar radiation via fluorescence, thereby protecting the holobiont from photoinhibition (Salih et al. 2000, Dove 2004). These mechanisms deal with avoidance of damage and/or damage in the photosystems themselves. Other organic compounds such as a wide range of antioxidants and FPs can also be produced in the holobiont to counteract consequences if damage of the photosynthetic apparatus could not be avoided. Antioxidants play an especially important role in oxidative stress resistance (Lesser 2006; Chapters 3,4) and a more detailed account of their function and diversity will be given in section 6.2. 
These different mechanisms work separately and/or together to protect the photosynthetic apparatus, and cell-structure and -function from any stress-induced damage. Dissimilar Symbiodinium types might emphasize different protective mechanisms, with some making more use of NPQ while others might have a higher production of antioxidants, for instance. This variability is founded on the immense genetic diversity within the genus Symbiodinium (LaJeunesse 2001, Coffroth and Santos 2005, Pochon and Gates 2010). Scleractinian corals, as well as anemones, can associate with seven of the nine known Symbiodinium clades, and each clade has a large number of sub-clades, or types, many with only small differences in their genetic code (Baker 2003, Pochon et al. 2010). It has long been known that even closely related types can vary in their stress responses (Sampayo et al. 2008), but responses are not only influenced by the algal genetic code, but also by thermal history (of both host and symbiont) and geographic origin (Rowan and Knowlton 1995, Baird et al. 2009, Barshis et al. 2010). The importance of the latter has been demonstrated in the current study. When subjected to stress, Symbiodinium types that originated from higher latitudes appeared to be more tolerant than types from lower latitudes (Chapters 2, 3, 4). The reason for this could be that Symbiodinium types at higher latitudes have to be able to deal with temperatures that can often be higher or lower (and more variable) than temperatures on a typical tropical coral reef (Kleypas et al. 1999). For instance, average surface sea temperatures (SST) in Hawaii range from 24 to $27{ }^{\circ} \mathrm{C}$ (NOAA) throughout the year, whilst SST in Okinawa can reach up to $28-30{ }^{\circ} \mathrm{C}$ in summer and fall to $21-23{ }^{\circ} \mathrm{C}$ in winter (www.surf-forecast.com). Other high latitude reefs have similar SST ranges, suggesting that not only the extreme values but the variability and the range of temperature are important. With thermal conditions that often change naturally, for example with season, the Symbiodinium types from these reefs might be better prepared to counteract a change that is more marked; for example, types from higher latitudes such as A1 (Gulf of Aqaba) and E1 (Okinawa) from this study appeared to be very tolerant to oxidative stress. This theory is corroborated by the extreme cases of Symbiodinium californium in culture and type A1 freshly isolated from the anemone Anthopleura aureoradiata from Wellington, New Zealand. Not only are both types originally from high latitude temperate environments with a wide average temperature range of $13-20{ }^{\circ} \mathrm{C}$ (NOAA) and 8-19 ${ }^{\circ} \mathrm{C}$ (Greater Wellington Regional Council), respectively, but they originate from the intertidal zone. This combination results in an especially variable environment with large variations in many abiotic parameters over the year. These 
parameters include temperature, irradiance and daylight hours, nutrients and intermittent desiccation during low tide; moreover, temperatures may approach or even reach freezing levels during winter (Muller-Parker and Davy 2001, Muller-Parker et al. 2007, Gibbons 2008, Bingham et al. 2011). It appears that under these extreme natural conditions, the Symbiodinium types have evolved to be more tolerant towards thermal as well as oxidative stress. They have probably developed a wider range of the aforementioned protective mechanisms to be prepared for a rapid change in external conditions.

While it seems that geographic origin and thermal history influence the stress tolerance of Symbiodinium, predictions about clade- or type-specific differences need to be made with caution. Nevertheless, in the current study clades A and E were generally more stress tolerant than clades $\mathrm{B}$ and $\mathrm{F}$, even though their responses to thermal and oxidative stress were slightly different (Chapters 2, 3). In stress experiments with three types of the diverse clade C from the Great Barrier Reef, C15 was found to be generally more tolerant than types $\mathrm{C} 1$ and $\mathrm{C} 3$, with type $\mathrm{C} 1$ being the most susceptible (Chapter 5); these results were consistent with those of Fisher et al. (2012). The reason or need for these differences, given that these symbionts were collected from a relatively small area, are unclear. However, thermal history might still have varied due to the coral host's growth form (Fisher 2006, Kelley 2009). Indeed, the host's influence on the dinoflagellate's photosynthetic physiology during stress was apparent in the current study, as type C15 responded quite differently to thermal stress in the two coral hosts, Montipora digitata and Porites cylindrica.

It is useful to conduct research on Symbiodinium cells in culture to observe treatment effects uncoupled from any host influence. This can give a clearer picture of where, inside the dinoflagellate cell, damage is caused precisely and what innate stress responses are triggered as a consequence (Robison and Warner 2006). At the same time, it should be remembered that it is not a realistic representation of what might occur in the intact symbiosis under the same stress conditions. The host can provide protection for its symbionts in several ways, for example by influencing MAA production (Shick and Dunlap 2002), generating fluorescent pigments and antioxidants, or simply by its speciesspecific growth form which could facilitate self-shading of symbiont cells (Baird et al. 
2009). These host-related factors can have an impact on the cell's health during stress (Chapter 5). Therefore, as host and symbiont both contribute to a holobiont's stress response, it would be valuable to corroborate any laboratory data with similar experiments in the field.

\subsection{Protection from ROS - the special role of antioxidants}

As discussed earlier, Symbiodinium cells as well as their cnidarian hosts have different mechanisms to protect the photosynthetic apparatus from damage caused by high light or temperature (Bhagooli et al. 2008, Middlebrook et al. 2008). Still, ROS are constantly being produced inside the algal cells and can potentially cause damage to the holobiont (Halliwell and Gutteridge 1989, Mittler 2002). The intracellular production of antioxidants is one of the most important mechanisms by which the algal cells can protect themselves from ROS (Lesser 2006).

There are a number of non-enzymatic and enzymatic antioxidants, with the latter often being described as the first line of defence against ROS. Enzymatic antioxidants include SOD which reduces $\mathrm{O}_{2}{ }^{-}$to $\mathrm{H}_{2} \mathrm{O}_{2}$ (Veljović-Jovanović 1998, Asada 2000). However, if the end-product, $\mathrm{H}_{2} \mathrm{O}_{2}$, accumulates inside the cell it will have an inhibitory effect on enzyme activity (Cheng and Song 2006). This could have contributed to the patterns seen in the current study (Chapter 2-5), though $\mathrm{H}_{2} \mathrm{O}_{2}$ is typically reduced by antioxidant enzymes APX and CAT to water and oxygen (Venn et al. 2008). While not antioxidants, FPs produced by the coral host also have very high $\mathrm{H}_{2} \mathrm{O}_{2}$ scavenging activities and should be considered when studying stress in holobionts (Palmer et al. 2009, 2010; Chapter 5). Singlet oxygen $\left({ }^{1} \mathrm{O}_{2}\right)$ is removed by several non-enzymatic antioxidants, $\alpha$-tocopherol, glutathione (GSH) and carotenoids (Venn et al. 2008).

Antioxidants reduce ROS concentrations and prevent oxidative stress inside the algal cell (Downs et al. 2002, Liñán-Cabello et al. 2009). While the processes are simple, interpretation of antioxidant measurements in vivo are not. When looking at the total antioxidant potential in cultured Symbiodinium cells stressed with $\mathrm{H}_{2} \mathrm{O}_{2}$, two independent 
Chapter 6

assays (FRAP, CAA) found similar results (Chapter 4). They indicated increasing antioxidant concentration with increasing $\mathrm{H}_{2} \mathrm{O}_{2}$ concentration in two Symbiodinium types (A1 and F1-H) while antioxidants decreased in a third type (E1-O). These results could indicate that the former two types were able to increase their antioxidant potential due to the increase in stress and were perhaps more tolerant to oxidative stress than was type E1-O. On the one hand, this was supported by chlorophyll fluorescence data from earlier experiments, which showed type $\mathrm{A} 1$ to be more tolerant to $\mathrm{H}_{2} \mathrm{O}_{2}$ induced oxidative stress than type E1-O (Chapter 2). On the other hand, type F1-H had shown very little tolerance to the added $\mathrm{H}_{2} \mathrm{O}_{2}$ (Chapter 2) while being able to up-regulate antioxidants in response to the same stressor (Chapter 4; Table 6.1); unfortunately, this contradiction cannot be explained with the available data sets. In contrast, type E1-O might have been more susceptible to stress, as it did not have the ability to up-regulate its antioxidant production. However, it might also be that antioxidants generated in type E1-O were used more efficiently than in the other two types, as suggested by the decrease in antioxidant concentration in Chapter 4 and the rapid decrease in ROS concentration in Chapter 3 (Table 6.1). High turnover rates could point to E1-O being, in fact, a tolerant Symbiodinium type; this was corroborated by data from earlier experiments in which chlorophyll fluorescence data showed E1-O to be tolerant of oxidative stress (Chapter 2).

Interpretation of changes in the concentrations of specific antioxidants (SOD, APX) in Symbiodinium cells in hospite should also be viewed cautiously (Chapter 5). There was a strong up-regulation of APX, as well as upstream SOD production during oxidative stress experiments with whole corals, while little change in antioxidant activity was observed during thermal stress experiments; this does not necessarily mean that algal cells were not stressed by increasing temperature. Instead, antioxidants might have been continuously produced and efficiently used during this longer-term, moderate thermal stress, with temperatures 2-3 ${ }^{\circ} \mathrm{C}$ higher than reached during summer months (Berkelmans 2002). $\mathrm{H}_{2} \mathrm{O}_{2}$ was added to the holobionts in concentrations much higher than experienced under natural conditions (Yuan and Shiller 1999); up-regulation of antioxidants, especially APX which reduces $\mathrm{H}_{2} \mathrm{O}_{2}$, appeared to be the immediate response to cope with the sudden and extreme stress in all Symbiodinium types. It appears that the C15 type, which was most tolerant to $\mathrm{H}_{2} \mathrm{O}_{2}$ addition in comparison to the other Heron Island types as indicated from the $\mathrm{F}_{\mathrm{v}} / \mathrm{F}_{\mathrm{m}}$ 
data, was also the one that increased its antioxidant activity more. Interpreting antioxidant development with respect to stress tolerance is even more difficult in Symbiodinium cells in hospite than in culture. Not only do the earlier considerations need to be taken into account, but the coral host influences the bleaching response as well; by generating its own antioxidants, or at least influencing their production in the algae (Ferrier-Pagès et al. 2007, Baird et al. 2009), the host can possibly diminish the stress on its symbionts and consequently change their response.

It appears that measuring antioxidant activity alone cannot provide a clear picture of the algal cell's stress tolerance. Therefore, it would be useful to measure photosynthetic efficiency via PAM, antioxidant activity and other protective mechanism at the same time.

\subsection{Relationship between thermal and oxidative stress}

The Symbiodinium stress response is influenced by many different factors, such as photoprotective mechanisms, antioxidant production and cell structure. These factors are determined by the algae's transcriptome and therby protein expression, probably in combination with environmental factors as discussed earlier. For this study, the effects of both thermal and oxidative stress on photosynthetic capacity of different Symbiodinium types were tested. Subsequently, antioxidant production as a proxy for the algal cell's oxidative stress resistance was measured. Some types were found to be tolerant to increasing temperature and $\mathrm{H}_{2} \mathrm{O}_{2}$ concentration, as was seen for the temperate cultured S. californium and freshly isolated Symbiodinium A1 from a New Zealand anemone, suggesting a possible link between thermal and oxidative stress. Other types displayed a high tolerance to increasing temperature but were very susceptible to oxidative stress, like the tropical type F1-H from Hawaii and type B2 from Bermuda. Other types showed opposing stress responses, with lower tolerance to temperature and higher tolerance to oxidative stress induced by $\mathrm{H}_{2} \mathrm{O}_{2}$; for example type A1 from the Gulf of Aqaba (Chapters 2, 3; Table 6.1). It therefore appears that it is not necessarily possible to predict a Symbiodinium type's response to one stress by knowing its susceptibility to another. At the same time, as discussed above, antioxidant production during either stress is sometimes 
Chapter 6

difficult to interpret with respect to the cell's oxidative stress resistance. In light of these different stress responses, a key question is how oxidative stress is related to thermal stress.

It is widely considered that ROS are toxic by-products of aerobic metabolism, generated during stress in Symbiodinium cells and which, if not removed quickly enough, can lead to irreversible damage in cell structure and function. Ultimately ROS may induce the expulsion of the algal cells from the cnidarian host (Halliwell and Gutteridge 1989, Lesser 2006). This sequence of events is supported by a number of studies (Sies 1997, Tchernov et al. 2004, Smith et al. 2005, Suggett et al. 2008), however it is often forgotten that even under non-stressful conditions, ROS are constantly being produced at low levels inside Symbiodinium cells and may function as signalling molecules (Mullineaux et al. 2000, Mittler 2002, Apel and Hirt 2004).

During non-stressful conditions, ROS are part of the redox signal transduction pathway, while during stress they act as secondary messengers; they activate cell processes such as defence pathways and programmed cell death, and adjust gene expression and cell structure as part of the stress response (Mittler 2002, Wagner et al. 2005, Lesser 2006, Mittler et al. 2011). Therefore, a higher generation of ROS with accompanying higher antioxidant levels under non-stressful conditions might enable Symbiodinium cells to produce a faster response to thermal and light stress. This may have been the case in the temperate S. californium, which contained higher ROS concentrations than the other types, even at the start of experiments (Chapter 3), and was relatively resistant to stress.

These considerations suggest that dissimilar Symbiodinium types might have different strategies to deal with stress (Fig. 6.1). Some cells appear to put more energy into mechanisms that prevent damage to the photosynthetic apparatus and photoinhibition, such as D1 repair or NPQ, than into mechanisms that remove ROS. This implies that increased ROS generation needs to be avoided as long as possible in these types. Other types might accept damage to the photosystems and ROS generation while more metabolic energy is used for defence mechanisms that neutralise ROS, such as antioxidants. This might suggest that short-term stress to the photosynthetic apparatus is necessary to be better prepared for 
Lower latitudes ..............................................................................higher latitudes

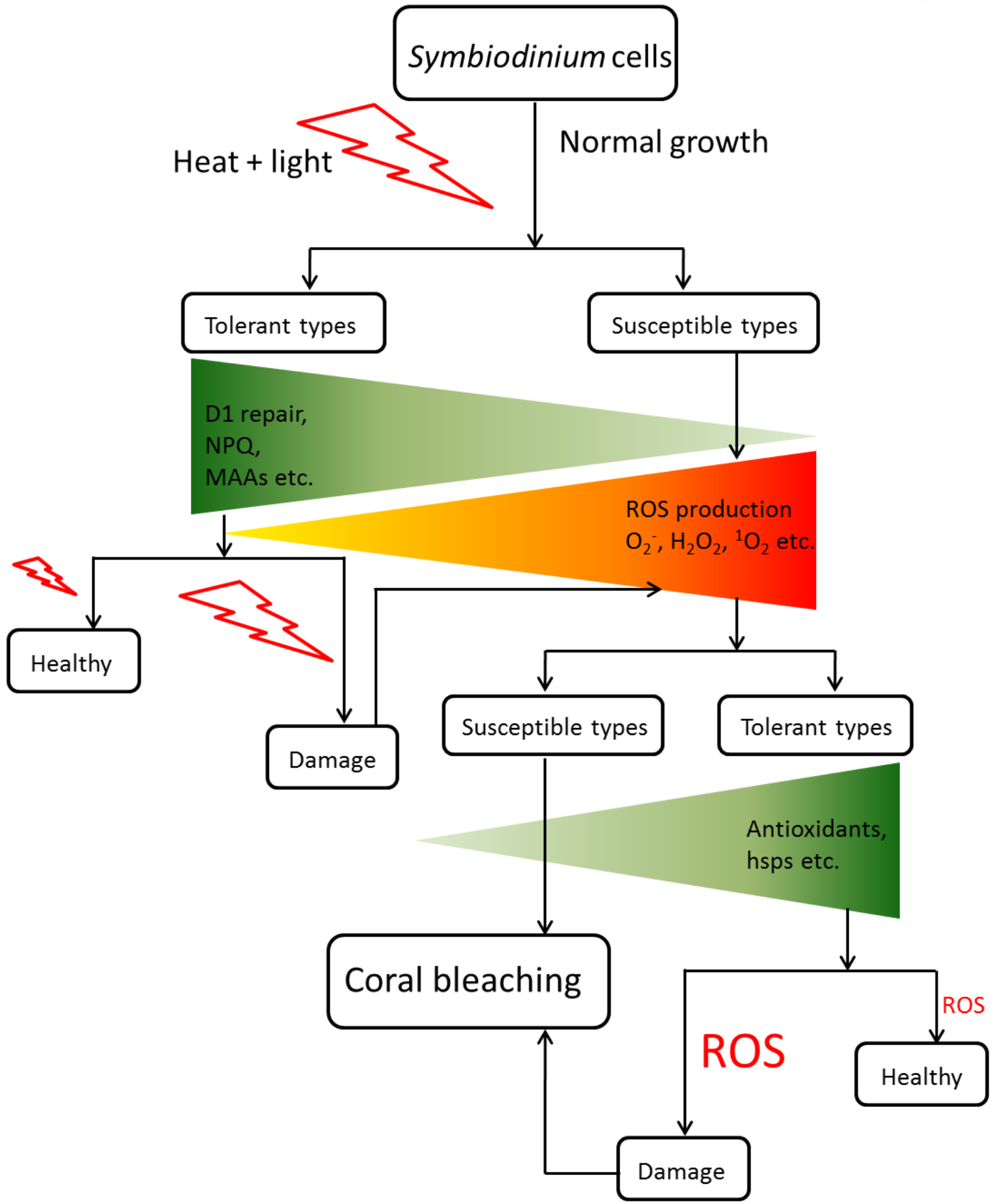

Figure 6.1: Simplified schematic figure depicting the relationship between thermal and oxidative stress in tolerant and susceptible types of Symbiodinium sp across latitudes. Thermal stress is presented as lightning bolts, oxidative stress as ROS (size indicates severity and duration of stress). In triangles, colour gradient represents different concentrations of triangle content. 
Chapter 6

long-term oxidative stress. The first strategy might be applicable for Symbiodinium types in stable environments like tropical coral reefs. Any variation in external conditions such as temperature would normally be only short term, which these types (e.g. F1-H) would be prepared for. The second strategy might be more useful for Symbiodinium types in more variable environments like higher latitude coral or rocky reefs. External conditions vary regularly and stress might be longer term, so damage to the photosynthetic apparatus would most definitely occur. Therefore, types that follow this strategy might invest relatively less in photoprotective mechanisms and allow for some ROS generation, while focusing their energetic resources on ROS removal, for example by antioxidants. This might have been the case in type A1 from the Gulf of Aqaba. In comparison this is the riskier strategy, as there might be a fine line between the amounts of photoinhibition and ROS a cell can counter via antioxidants, and the amounts that lead to algal cell death and bleaching. Still, accepting some photoinhibition and ROS generation for the 'greater good' of cell survival could be the better strategy for Symbiodinium types in these environments.

Currently, the natural variations in environmental conditions are intensified and made more problematic by anthropogenic climate change. Surface seawater temperatures (SST) are higher on average, and also show more regular and extreme outliers (Donner 2009). These previously unencountered conditions might lead to too much ROS generation for cells of the first strategy to handle, due to their relatively limited antioxidant production. At the same time, it might also lead to strong photoinhibition in cells that utilise the second strategy. In both cases, this might lead to irreversible damage inside the algal cell and bleaching. The best chances of survival might lie with those Symbiodinium types that use a combination of the two strategies, such as S. californium and Symbiodinium A1 from New Zealand. They appear to be well prepared for either stress due to the variable environments experienced at their geographic origins.

\subsection{The future of coral reefs}

As described in earlier chapters, Symbiodinium types have different strategies to deal with external and consequent internal stress. These strategies might have slowly developed over 
thousands of years and are adapted to suit their respective environments (Berkelmans 2002, Sampayo et al. 2008). Subsequently, many coral reefs have formed with an immense diversity of cnidarian-dinoflagellate associations and local differences (Jackson 1991, LaJeunesse 2001, Baker 2003). Until now, lower latitude coral reefs were considered optimal due to their stable conditions. Optimum conditions are traditionally associated with a higher biodiversity, while increasing latitude in combination with decreasing temperature leads to a decrease in diversity (Guinotte et al. 2003). Extreme cases are marginal reefs, which are defined as those where conditions are close to the environmental thresholds for coral survival (Kleypas et al. 1999, Harriott and Banks 2002). Amongst these thresholds are low and high temperature, salinity and nutrient levels, as well as light and aragonite availability (Kleypas et al. 1999). Accordingly, marginal environments also exist at lower latitudes in areas not suitable for reef formation due to runoff, upwelling or river deltas (Perry and Larcombe 2003).

This distribution of coral reefs and their respective diversities might dramatically change in the future due to anthropogenic threats; in particular, increasing SSTs due to global warming might change the face of coral reefs (Hughes et al. 2003, Hoegh-Guldberg 2007, Donner 2009). The areas that are likely to be affected first lie in the tropical belt, which typically has stable conditions close to the upper thermal limits of corals. Even a slight increase in temperature could cause damage to the holobiont and might lead to bleaching. But, as discussed earlier, responses vary between Symbiodinium types and also coral host species (Chapter 2-5). This could point to a future in which certain Symbiodinium types might be prevalent due to their higher stress tolerance, as has been proposed for Symbiodinium clade D in the past (Baker et al. 2004, Berkelmans and Van Oppen 2006). Additionally, certain host-symbiont pairings might have an advantage over other combinations due to their overall stress resistance, but also higher recruitment and growth rates (Donner 2009). Therefore, these associations might increase in numbers, and previously diverse coral reefs could shift into a mono-specific state. Coral species that have been observed to be less affected by disturbances mainly include encrusting species such as Montipora spp. (consistent with the behaviour of the Symbiodinium type studied here Chapter 5), and several massive species (Hughes and Connell 1999). 
Chapter 6

Another future scenario is the shift from predominantly tropical coral reefs to higher latitude or marginal reef communities (Guinotte et al. 2003, Wicks 2009). These reefs consist of coral species with symbionts accustomed to variable environmental conditions or that even host multiple symbiont types to cope with fluctuations (LaJeunesse et al. 2004); coral hosts from these environments might therefore have a natural advantage during future change. This has also been shown in the current study with Symbiodinium types from variable higher latitude environments displaying greater tolerances to thermal and oxidative stress. Due to being situated in colder climates, high latitude reefs might also serve as refuges for tropical reef species when their natural homes become too warm (Riegl and Piller 2003). Therefore, while some high latitude reefs already display a high diversity today, like Lord Howe Island, Australia (Harriot et al. 1995), others might become more diverse in the future.

In conclusion, this work has shown that stress elicits a wide range of responses within the genus Symbiodinium, which is dependent on type, state of symbiosis and thermal history. I have contributed new insight into the complex relationship between thermal and oxidative stress; it was demonstrated that knowing a Symbiodinium type's response to one stress does not enable us to predict its tolerance to another. It appears that Symbiodinium types vary in their intracellular stress pathways with some types maximising initial protection against stress while others maximise repair but remain somewhat susceptible. Repair mechanisms might include antioxidant generation whose significance remains elusive. The work presented here represents an important first step and future research should include other protective mechanisms, both for photoprotection such as D1 repair, NPQ and thylakoid membrane composition, as well as ROS scavenging mechanisms, for comparison and to provide a more complete picture of the cellular events that occur. The relationship between thermal and oxidative stress should be further investigated and the theories presented here about the influence of geographic origin tested by examining a wider range of Symbiodinium types from across a latitudinal gradient and other different habitats.

The ongoing rise in seawater temperature induced by climate change leads to coral bleaching events and might change the face of coral reefs for ever. Results from this research shed light on intracellular processes occurring during bleaching events and offer a 
Chapter 6

more complete picture of initial stress response leading to ROS build-up and subsequent stress resistance through antioxidant generation. Lessons learnt from algal cells in culture and freshly isolated cells are likely to provide important parallels to Symbiodinium cells in hospite. Ultimately, this might help explain coral bleaching susceptibilities and the important role that Symbiodinium play therein. 


\section{References}

Abrego D., Ulstrup K.E., Willis B.L., Van Oppen M.J.H. (2008) Species-specific interactions between algal endosymbionts and coral hosts define their bleaching response to heat and light stress. Proceedings of the Royal Society B 275, 2273-2282.

Ainsworth T.D., Hoegh-Guldberg O., Heron S.F., Skirving W.J., Leggat W. (2008) Early cellular changes are indicators of pre-bleaching thermal stress in the coral host. Journal of Experimental Marine Biology and Ecology 364, 63-71.

Allan A.C. and Fluhr R. (1997) Two distinct sources of elicited reactive oxygen species in tobacco epidermal cells. The Plant Cell 9, 1559-1572.

Allen R.D. (1995) Dissection of oxidative stress tolerance using transgenic plants. Plant Physiology 107, 1049-1 054.

Alscher R.G., Donahue J.L., Cramer C.L. (1997) Reactive oxygen species and antioxidants: Relationships in green cells. Physiologia Plantarum 100, 224-233.

Alscher R.G., Erturk N., Heath L.S. (2002) Role of superoxide dismutases (SODs) in controlling oxidative stresss in plants. Journal of Experimental Botany 53(372), 13311341

Apel K. and Hirt H. (2004) Reactive oxygen species: Metabolism, oxidative stress, and signal transduction. Annual Review of Plant Biology 55, 373-99.

Asada K. (2000) The water-water cycle as alternative photon and electron sinks. Philosophical Transactions of the Royal Society B 355, 1419-1431.

Ashtamker C., Kiss V., Sagi M., Davydov O., Fluhr R. (2007) Diverse subcellular locations of cryptogein-induced reactive oxygen species production in tobacco bright yellow-2 cells1. Plant Physiology 143, 1817-1826. 
References

Badger M.R., Von Caemmerer S., Ruuska S., Nakano H. (2000) Electron flow to oxygen in higher plants and algae: rates and control of direct photoreduction (Mehler reaction) and rubisco oxygenase. Philosophical Transactions of the Royal Society B. 355, $1433-1446$.

Baird A.H., Bhagooli R., Ralph P.J. and Takahashi S. (2009) Coral bleaching: the role of the host. Trends in Ecology and Evolution 24(1), 16-20.

Baker A.C. (2003) Flexibility and specificity in coral-algal symbiosis: Diversity, Ecology, and Biogeography of Symbiodinium. Annual Review of Ecology, Evolution, and Systematics 34, 661-689.

Baker A.C., Starger C.J., McClanahan T.R., Glynn P.W. (2004) Corals' adaptive response to climate change. Nature 430, 741.

Baroli I., Gutman B.L., Ledford H.K., Shin J.W., Chin B.L., Havaux M., Niyogi K.K. (2004) Photo-oxidative stress in a xanthophyll-deficient mutant of Chlamydomonas. The Journal of Biological Chemistry 279, 6337-6344.

Barshis D.J., Stillman J.H., Gates R.D., Toonen R.J., Smith L.W., Birkeland C. (2010) Protein expression and genetic structure of the coral Porites lobata in an environmentally extreme Samoan back reef: does host genotype limit phenotypic plasticity? Molecular Ecology 19, 1705-1720.

Bartosz G. (2006) Use of spectroscopic probes for detection of reactive oxygen species. Clinica Chimica Acta 368, 53-76.

Belda-Baillie C.A., Baillie B.K., Maruyama T. (2002) Specificity of a model cnidariandinoflagellate symbiosis. The Biological Bulletin 202, 74-85.

Bellwood D.R., Hughes T.P., Folke C., Nyström M. (2004) Confronting the coral reef crisis. Nature 429, 827-833. 
Benzie J.A.H., Haskell A., Lehman H. (1995) Variation in the genetic composition of coral (Pocillopora damicornis and Acropora palifera) populations from different reef habitats. Marine Biology 121, 731-739.

Benzie I.F.F. and Strain J.J. (1996) The Ferric Reducing Ability of Plasma (FRAP) as a measure of "Antioxidant Power"': The FRAP Assay. Analytical Biochemistry 239, 70-76.

Berkelmans R. (2002) Time-integrated thermal bleaching thresholds of reefs and their variation on the Great Barrier Reef. Marine Ecology Progress Series 229, 73-82.

Berkelmans R. and Oliver J.K. (1999) Large-scale bleaching of corals on the Great Barrier Reef. Coral Reefs 18, 55-60.

Berkelmans R. and Van Oppen M.J.H. (2006) The role of zooxanthellae in the thermal tolerance of corals: a 'nugget of hope' for coral reefs in an era of climate change. Proceedings of the Royal Society B 273, 2305-2312.

Beyer W.F. Jr and Fridovich I. (1987) Assaying for superoxide dismutase activity: Some large consequences of minor changes in conditions. Analytical Biochemistry 161, 559-566.

Bhagooli R., Baird A.H., Ralph P.J. (2008) Does the coral host protect its algal symbionts from heat and light stresses? Proceedings of the 11th International Coral Reef Symposium, Ft. Lauderdale, Florida.

Bingham B.L., Freytes I., Emery M., Dimond J., Muller-Parker G. (2011) Aerial exposure and body temperature of the intertidal sea anemone Anthopleura elegantissima. Invertebrate Biology 130(4): 291-301.

Black N.A., Voellmy R., Szmant A.M. (1995) Heat shock protein induction in Montastraea faveolata and Aiptasia pallida exposed to elevated temperatures. The Biological Bulletin 188, 234-240. 
Bongaerts P., Ridgway T., Sampayo E.M., Hoegh-Guldberg O. (2010) Assessing the 'deep reef refugia' hypothesis: focus on Caribbean reefs. Coral Reefs 29, 309-327.

Borell E.M. and Bischof K. (2008) Feeding sustains photosynthetic quantum yield of a scleractinian coral during thermal stress. Oecologia 157, 593-601.

Bouchard J.N. and Yamasaki H. (2008) Heat stress stimulates nitric oxide production in Symbiodinium microadriaticum: a possible linkage between nitric oxide and the coral bleaching phenomenon. Plant and Cell Physiology 49(4), 641-652.

Boucher D.H. (1985) The Biology of Mutualism: Ecology and Evolution. Oxford University Press Inc. New York, 13-18.

Bradford M.M. (1976) A rapid and sensitive method for the quantitation of microgram quantities of protein utilizing the principle of protein-dye binding. Analytical Biochemistry $72,248-254$.

Brown B.E., Dunne R.P., Goodson M.S., Douglas A.E. (2002) Experience shapes the susceptibility of a reef coral to bleaching. Coral Reefs 21, 119-126.

Buddemeier R.W. and Fautin D.G. (1993) Coral bleaching as an adaptive mechanism. BioScience 43(5), 320-326.

Butow B.J., Wynne D., Tel-Or E. (1997a) Antioxidant protection of Peridinium gatunense in Lake Kinneret: seasonal and daily variation. Journal of Phycology 33, 780786.

Butow B.J., Wynne D., Tel-Or E. (1997b) Superoxide dismutase activity in Perdinium gatunense an Lake Kinneret: Effect of light regime and carbon dioxide. Journal of Phycology 33, 787-793. 
Buxton L., Takahashi S., Hill R., Ralph P.J. (2011) Variability in the primary site of photosynthetic damage in Symbiodinium sp. (Dinophyceae) exposed to thermal stress. Journal of Phycology 48(1), 117-126.

Chen C.A., Yang Y.-W., Wei N.V., Tsai W-S., Fang L.-S. (2005) Symbiont diversity in scleractinian corals from tropical reefs and subtropical non-reef communities in Taiwan. Coral Reefs 24, 11-22.

Chen M-C., Cheng Y.-M., Hong M.-C., Fang L.-S. (2004) Molecular cloning of Rab5 (ApRab5) in Aiptasia pulchella and its retention in phagosomes harboring live zooxanthellae. Biochemical and Biophysical Research Communications 324, 1024-1033.

Cheng H.-Y. and Song S.-Q. (2006) Species and organ diversity in the effects of hydrogen peroxide on superoxide dismutase activity in vitro. Journal of Integrative Plant Biology 48(6), 672-678.

Choo K.-S., Snoeijs P., Pedersén M. (2004) Oxidative stress tolerance in the filamentous green algae Cladophora glomerata and Enteromorpha ahlneriana. Journal of Experimental Marine Biology and Ecology 298, 111 - 123.

Coffroth M. A., Santos S. R., Goulet T. L. (2001) Early ontogenetic expression of specificity in a cnidarian-algal symbiosis. Marine Ecology Progress Series 222, 85-96.

Coffroth M.A. and Santos S.R. (2005) Genetic diversity of symbiotic dinoflagellates in the genus Symbiodinium. Protist 156, 19-34.

Coffroth M.A., Santos S.R., Goulet T.L. (2001) Early ontogenetic expression of specificity in a cnidarian-algal symbiosis. Marine Ecology Progress Series 222, 85-96.

Correa A.M.S. and Baker A.C. (2008) Understanding diversity in coral-algal symbiosis: a cluster-based approach to interpreting fine-scale genetic variation in the genus Symbiodinium. Coral Reefs 28(1), 81-93. 
References

Crawley A., Kline D.I., Dunn S., Anthony K., Dove S. (2010) The effect of ocean acidification on symbiont photorespiration and productivity in Acropora formosa. Global Change Biology 16, 851-863.

Császár N.B.M., Seneca F.O. and Van Oppen M.J.H. (2009). Variation in antioxidant gene expression in the scleractinian coral Acropora millepora under laboratory thermal stress. Marine Ecology Progress Series 392, 93-102.

Davies P.S. (1991) Effect of daylight variations on the energy budgets of shallow-water corals. Marine Biology 108, 137-144.

Davy S.K., Allemand D., Weis V.M (2012) The cell biology of cnidarian-dinoflagellate symbiosis. Microbiology and Molecular Biology Reviews 76(2), 229-261.

Donahue J.L., Okpodu C.M., Cramer C.L., Grabau E.A., Alscher R.G. (1997) Responses of antioxidants to paraquat in pea leaves. Plant Physiology 113, 249-257.

Donner S.D. (2009) Coping with commitment: projected thermal stress on coral reefs under different future scenarios. PLoS ONE 4(6), e5712.

Douglas A.E. (2003) Coral bleaching-how and why? Marine Pollution Bulletin 46, 385392.

Dove S. (2004) Scleractinian corals with photoprotective host pigments are hypersensitive to thermal bleaching. Marine Ecology Progress Series 272, 99-116.

Dove S., Ortiz J.C., Enriquez S., Fine M., Fisher P., Iglesias-Prieto R., Thornhill D., Hoegh-Guldberg O. (2006) Response of holosymbiont pigments from the scleractinian coral Montipora monasteriata to short-term heat stress. Limnology and Oceanography 51(2), 1149-1158. 
Downs C.A., Fauth J.E., Halas J.C., Dustan P., Bemiss J., Woodley C.M. (2002) Oxidative stress and seasonal coral bleaching. Free Radical Biology \& Medicine 33, 533543.

Downs C.A., Mueller E., Phillips S., Fauth J.E., Woodley C.M. (2000) A molecular biomarker system for assessing the health of coral (Montastraea faveolata) during heat stress. Journal of Marine Biotechnology 2, 533-544.

Driever S.M., Fryer M.J., Mullineaux P.M. Baker N.R. (2009) Imaging of reactive oxygen species in vivo. In T. Pfannschmidt (ed.), Plant Signal Transduction 479, 109-116.

Drummond A.J., Ashton B., Buxton S., Cheung M., Cooper A., Duran C., Field M., Heled J., Kearse M., Markowitz S., Moir R., Stones-Havas S., Sturrock S., Thierer T., Wilson A. (2011) Geneious v5.4.

Dunn S.R., Thomason J.C., Le Tissier M.D.A., Bythell J.C. (2004) Heat stress induces different forms of cell death in sea anemones and their endosymbiotic algae depending on temperature and duration. Cell Death and Differentiation 11, 1213-1222.

Dunn S.R. and Weis V.M. (2009) Apoptosis as a post-phagocytic winnowing mechanism in a coral-dinoflagellate mutualism. Environmental Microbiology 11(1), 268-276. 
Eakin C.M., Morgan J.A., Heron S.F., Smith T.B., Liu G., Alvarez-Filip L., Baca B., Bartels E., Bastidas C., Bouchon C., Brandt M., Bruckner A.W., Bunkley-Williams L., Cameron A., Causey B.D., Chiappone M., Christensen T.R.L., Crabbe M.J.C., Day O., de la Guardia E., Díaz-Pulido G., DiResta D., Gil-Agudelo D.L., Gilliam D.S., Ginsburg R.N., Gore S., Guzmán H.M., Hendee J.C., Hernández-Delgado E.A., Husain E., Jeffrey C.F.G., Jones R.J., Jordán-Dahlgren E., Kaufman L.S., Kline D.I., Kramer P.A., Lang J.C., Lirman D., Mallela J., Manfrino C., Maréchal J.-P., Marks K., Mihaly J., Miller W.J., Mueller E.M., Muller E.M., Orozco Toro C.A., Oxenford H.A., Ponce-Taylor D., Quinn N., Ritchie K.B., Rodríguez S., Ramírez A.R., Romano S., Samhouri J.F., Sánchez J.A., Schmahl G.P., Shank B.V., Skirving W.J., Steiner S.C.C., Villamizar E., Walsh S.M., Walter C., Weil E., Williams E.H., Roberson K.W., Yusu Y. (2010) Caribbean corals in crisis: record thermal stress, bleaching, and mortality in 2005. PLoS ONE 5(11), e13969.

Eckert R. and Randall D. (1978) Animal Physiology. San Francisco, W.H. Freeman and Company.

Eruslanov E. and Kusmartsev S. (2010). Identification of ROS using oxidized DCFDA and flow-cytometry. In D. Armstrong (ed.) Advanced Protocols in Oxidative Stress II, Methods in Molecular Biology 594, 57-72.

Falkowski P.G. and Raven J.A. (2007) Aquatic photosynthesis. Princeton University Press, 2nd Edition.

Fang L.-S., Huang S.-P., Lin K.-L. (1997) High temperature induces the synthesis of heat-shock proteins and the elevation of intracellular calcium in the coral Acropora grandis. Coral Reefs 16, 127-131.

Fautin D.G. and Buddemeier R.W. (2004) Adaptive bleaching: a general phenomenon. Hydrobiologia 530/531, 459-467. 
Ferrier-Pagés C., Richard C., Forcioli D., Allemand D., Pichon M., Shick J.M. (2007) Effects of temperature and UV radiation increases on the photosynthetic efficiency in four Scleractinian coral species. The Biological Bulletin 213, 76-87.

Fine M., Meroz-Fine E., Hoegh-Guldberg O. (2005) Tolerance of endolithic algae to elevated temperature and light in the coral Montipora monasteriata from the southern Great Barrier Reef. The Journal of Experimental Biology 208, 75-81.

Fisher P.L. (2006) Investigating the photo-physiology of Symbiodinium sub-clades and its relationship to coral bleaching. Dissertation, University of Queensland, Brisbane, Australia, USA.

Fisher P.L., Malme M.K., Dove S. (2012) The effect of temperature stress on coralSymbiodinium associations containing distinct symbiont types. Coral Reefs 31, 473-485.

Fishman Y., Zlotkin E., Sher D. (2008) Expulsion of symbiotic algae during feeding by the green hydra - a mechanism for regulating symbiont density? PLoS ONE 3(7), e2603.

Fitt W.K. (2000) Cellular growth of host and symbiont in a cnidarian - zooxanthellar symbiosis. The Biological Bulletin 198, 110-120.

Fitt W.K. and Trench R.K. (1983) Endocytosis of the symbiotic dinoflagellate Symbiodinium microadraticum Freudenthal by endodermal cells of the Scyphistomae of Cassiopeia xamachana and resistance of the algae to host digestion. Journal of Cell Science 64, 195-212.

Fitt W.K., McFarland F.K., Warner M.E., Chilcoat G.C. (2000) Seasonal patterns of tissue biomass and densities of symbiotic dinoflagellates in reef corals and relation to coral bleaching. Limnology and Oceanography 45(3), 677-685.

Fitt W.K., Brown B.E., Warner M.E, Dunne R.P. (2001) Coral bleaching: interpretation of thermal tolerance limits and thermal thresholds in tropical corals. Coral Reefs 20, 51-65. 
Fitt W.K., Gates R.D., Hoegh-Guldberg O., Bythell J.C., Jatkar A., Grottoli A.G., Gomez M., Fisher P., Lajuenesse T.C., Pantos O., Iglesias-Prieto R., Franklin D. J., Rodrigues L.J., Torregiani J.M., van Woesik R., Lesser M.P. (2009) Response of two species of Indo-Pacific corals, Porites cylindrica and Stylophora pistillata, to short-term thermal stress: The host does matter in determining the tolerance of corals to bleaching. Journal of Experimental Marine Biology and Ecology 373, 102-110.

Flores-Ramírez L.A. and Liñán-Cabello M.A. (2007) Relationships among thermal stress, bleaching and oxidative damage in the hermatypic coral, Pocillopora capitata. Comparative Biochemistry and Physiology, Part C 146, 194-202.

Flors C. Fryer M.J., Waring J., Reeder B., Bechtold U., Mullineaux P.M., Nonell S., Wilson M.T. Baker N.R. (2006) Imaging the production of singlet oxygen in vivo using a new fluorescent sensor, Singlet Oxygen Sensor Green. The Journal of Experimental Botany 57, 1725-1734.

Foyer C.H. and Noctor G. (2005) Oxidant and antioxidant signalling in plants: a reevaluation of the concept of oxidative stress in a physiological context. Plant, Cell and Environment 28, 1056-1071.

Franklin D.J., Hoegh-Guldberg O., Jones R.J., Berges J.A. (2004) Cell death and degeneration in the symbiotic dinoflagellates of the coral Stylophora pistillata during bleaching. Marine Ecology Progress Series 722, 117-130.

Fransolet D., Roberty S., Plumier J.-C. (2012) Establishment of endosymbiosis: The case of cnidarians and Symbiodinium. Journal of Experimental Marine Biology and Ecology 420-421, 1-7.

Freudenthal H. (1962) Symbiodinium gen. nov. and Symbiodinium microadriaticum sp. nov., a zooxanthella: Taxonomy, life cycle, and morphology. Journal of Protozoology 9,45 . 
Fridovich I. (1998). Oxygen toxicity: a radical explanation. The Journal of Experimental Botany 201, 1203-1209.

Fryer M.J., Oxborough K., Mullineaux P.M., Baker N.R. (2002) Imaging of photooxidative stress responses in leaves. The Journal of Experimental Botany 53, 1249-1254.

Gates R. D., Baghdasarian G., Muscatine L. (1992). Temperature stress causes host cell detachment in symbiotic cnidarians: Implications for coral bleaching. The Biological Bulletin 182, 324-332.

Gates R. D., Hoegh-Guldberg O., McFall-Ngai M. J., Bil K. Y., Muscatine L. (1995) Free amino acids exhibit anthozoan "host factor" activity: They induce the release of photosynthate from symbiotic dinoflagellates in vitro. Proceedings of the National Academy of Science USA 92, 7430-7434.

GeoSymbio: http://sites.google.com/site/geosymbio/home

Gibbons C.L. (2008) Carbon flux in the temperate zooxanthellate sea anemone Anthopleura aureoradiata. Master Thesis. Victoria University of Wellington, New Zealand.

Gomes A., Fernandes E., Lima J.L.F.C. (2005) Fluorescence probes used for detection of reactive oxygen species. Journal of Biochemical and Biophysical Methods 65, 45-80.

Gorbunov M.Y., Kolber Z.S., Lesser M.P., Falkowski P.G. (2001) Photosynthesis and photoprotection in symbiotic corals. Limnology and Oceanography 46(1), 75-85.

Goulet T.L. and Coffroth M.A. (1997) A within colony comparison of zooxanthella genotypes in the Caribbean gorgonian Plexaura kuna. Proceeding 8th International Coral Symposium 2, 1331-1334.

Greater Wellington Regional Council: www.gw.govt.nz/Porirua-Harbour-at-ManaCruising-Club. 
Griffin S.P. and Bhagooli R. (2004) Measuring antioxidant potential in corals using the FRAP assay. Journal of Experimental Marine Biology and Ecology 302, 201- 211.

Griffin S.P., Bhagooli R., Weil E. (2006) Evaluation of thermal acclimation capacity in corals with different thermal histories based on catalase concentrations and antioxidant potentials. Comparative Biochemistry and Physiology, Part A 144, 155-162.

Grigg R.W. (1994) The international coral-reef initiative - Conservation and effective management of marine resources. Coral Reefs 13, 197-198.

Guinotte J.M., Buddemeier R.W., Kleypas J.A. (2003) Future coral reef habitat marginality: temporal and spatial effects of climate change in the Pacific basin. Coral Reefs 22, 551-558.

Halliwell B. and Gutteridge J.M.C. (1989) Free Radicals in Biology and Medicine (2 $2^{\text {nd }}$ edition). Oxford, Clarendon Press.

Harriott V.J. and Banks S.A. (2002) Latitudinal variation in coral communities in eastern Australia: a qualitative biophysical model of factors regulating coral reefs. Coral Reefs 21, 83-94.

Harriott V.J., Harrison P.L., Banks S.A. (1995) The Coral Communities of Lord Howe Island. Marine and Freshwater Research 46, 457-465.

Harrison P.L., Dalton S.J., Carroll A.G. (2011) Extensive coral bleaching on the world's southernmost coral reef at Lord Howe Island, Australia. Coral Reefs 30, 775.

Hennige S.J., McGinley M.P., Grottoli A.G., Warner M.E. (2011) Photoinhibition of Symbiodinium spp. within the reef corals Montastraea faveolata and Porites astreoides: implications for coral bleaching. Marine Biology 158, 2515-2526.

Hideg E., Kós P.B., Vass I. (2007) Photosystem II damage induced by chemically generated singlet oxygen in tobacco leaves. Physiologia Plantarum 131, 33-40. 
Higuchi T., Fujimura H., Arakaki T., Oomori T. (2008) Activities of antioxidant enzymes (SOD and CAT) in the coral Galaxea fascicularis against increased hydrogen peroxide concentrations in seawater. Proceedings of the 11th International Coral Reef Symposium, Ft. Lauderdale, Florida.

Hill R. and Ralph P.J. (2006) Photosystem II heterogeneity of in hospite zooxanthellae in scleractinian corals exposed to bleaching conditions. Photochemistry and Photobiology 82, 1577-1585.

Hill R. and Ralph P.J. (2007) Post-bleaching viability of expelled zooxanthellae from the scleractinian coral Pocillopora damicornis. Marine Ecology Progress Series 352, 137-144.

Hill R. and Ralph P.J. (2008) Impact of bleaching stress on the function of the oxygen evolving complex of zooxanthellae from Scleractinian corals. Journal of Phycology 44, 299-310.

Hill R., Frankart C., Ralph P.J. (2005) Impact of bleaching conditions on the components of non-photochemical quenching in the zooxanthellae of a coral. Journal of Experimental Marine Biology and Ecology 322, 83-92.

Hill R., Larkum A.W.D., Frankart C., Kühl M., Ralph P.J. (2004) Loss of functional Photosystem II reaction centres in zooxanthellae of corals exposed to bleaching conditions: using fluorescence rise kinetics. Photosynthesis Research 82, 59-72.

Hill R., Ulstrup K.E., Ralph P.J. (2009) Temperature induced changes in thylakoid membrane thermostability of cultured, freshly isolated, and expelled zooxanthellae from scleractinian corals. Bulletin of Marine Science 85(3), 223-244.

Hoegh-Guldberg O. (1999) Climate change, coral bleaching and the future of the world's coral reefs. Marine and Freshwater Research 50, 839-866.

Hoegh-Guldberg O. (2006) International concern over coral bleaching on the Great Barrier Reef. http://www.uq.edu.au/news/?article=8903. 
Hoegh-Guldberg O. and Smith G.J. (1989) The effect of sudden changes in temperature, light and salinity on the population density and export of zooxanthellae from the reef corals Stylophora pistillata Esper and Seriatopora hystrix Dana. J. Exp. Mar. Biol. Ecol. 129, 279-303.

Hoegh-Guldberg O., Mumby P.J., Hooten A.J., Steneck R.S., Greenfield P., Gomez E., Harvell C.D., Sale P.F., Edwards A.J., Caldeira K., Knowlton N., Eakin C.M., Iglesias-Prieto R., Muthiga N., Bradbury R.H., Dubi A., Hatziolos M.E. (2007) Coral reefs under rapid climate change and ocean acidification. Science 318, 1737-1742.

Huang D., Ou B., Prior R.L. (2005). The chemistry behind antioxidant capacity assays. Journal of Agricultural and Food Chemistry 53 (6), 1841-1856.

Hughes T.P. and Connell J.H. (1999) Multiple stressors on coral reefs: a long-term perspective. Limnology and Oceanography 44(3) Part 2: The Effects of Multiple Stressors on Freshwater and Marine Ecosystems, 932-940.

Hughes T.P., Baird A.H., Bellwood D.R., Card M., Connolly S.R., Folke C., Grosberg R., Hoegh-Guldberg O., Jackson J.B.C., Kleypas J., Lough J.M., Marshall P., Nyström M., Palumbi S.R., Pandolfi J.M., Rosen B., Roughgarden J. (2003) Climate change, human impacts, and the resilience of coral reefs. Science 301, 929-933.

Hughes T.P., Baird A.H., Dinsdale E.A., Moltschaniwskyj N.A., Pratchett M.S., Tanner J.E., Willis B.L. (2012) Assembly rules of reef corals are flexible along a steep climatic gradient. Current Biology 22, 736-741.

Hughes T.P., Bellwood D.R., Baird A.H., Brodie J., Bruno J.F., Pandolfi J.M. (2011) Shifting base-lines, declining coral cover, and the erosion of reef resilience: comment on Sweatman et al. (2011). Coral Reefs 30, 653-660.

Iglesias-Prieto R., Beltrán V.H., LaJeunesse T.C., Reyes-Bonilla H., Thome P.E. (2004) Different algal symbionts explain the vertical distribution of dominant reef corals in the eastern Pacific. Proceedings of the Royal Society B 271, 1757-1763. 
Iglesias-Prieto R., Matta J.L., Robins W.A., Trench R.K. (1992) Photosynthetic response to elevated temperature in the symbiotic dinoflagellate Symbiodinium microadriaticum in culture. Proceedings of the National Academy of Sciences 89, 1030210305.

Jackson J.B.C. (1991) Adaptation and diversity of reef corals. BioScience 41(7), 475-482.

Janknegt P.J., Rijstenbil J.W., Van de Poll W.H., Gechev T.S., Buma A.G.J. (2007) A comparison of quantitative and qualitative superoxide dismutase assays for application to low temperature microalgae. Journal of Photochemistry and Photobiology B: Biology 87, $218-226$.

Jeffrey S.W. and Humphrey G.F. (1975) New spectrophotometric equations for determining chlorophylls $a, b, \mathrm{c}_{1}$ and $\mathrm{c}_{2}$ in higher plants, algae and natural phytoplankton. Biochemie und Physiologie der Pflanzen 167, 191-194.

Jones A.M., Berkelmans R., van Oppen M.J.H., Mieog J.C., Sinclair W. (2008) A community change in the algal endosymbionts of a scleractinian coral following a natural bleaching event: field evidence of acclimatization. Proceedings of the Royal Society B 275, $1359-1365$.

Jones R.J., Hoegh-Guldberg O., Larkum A.W.D., Schreiber U. (1998) Temperatureinduced bleaching of corals begins with impairment of the $\mathrm{CO}_{2}$ fixation mechanism in zooxanthellae. Plant, Cell and Environment 21, 1219-1230.

Jones R.J., Ward S., Amri A.Y., Hoegh-Guldberg O. (2000) Changes in quantum efficiency of photosystem II of symbiotic dinoflagellates of corals after heat stress, and of bleached corals sampled after the 1998 Great Barrier Reef mass bleaching event. Marine and Freshwater Research 51, 63-71.

Kelley R. (2009) The Australian Coral Reef Society Coral Finder Indo Pacific., Townsville, Australia, Russell Kelley. 
Kerswell A.P. and Jones R.J. (2003) Effects of hypo-osmosis on the coral Stylophora pistillata: nature and cause of 'low-salinity bleaching'. Marine Ecology Progress Series $253,145-154$.

Kleypas J.A. and Yates K.K. (2009) Coral reefs and ocean acidification. Oceanography 22(4), 108-117.

Kleypas J.A., McManus J.W., Meñez L.A.B. (1999) Environmental limits to coral reef development: where do we draw the line? American Zoologist 39, 146-159.

Koike K., Jimbo M., Sakai R., Kaeriyama M., Muramoto K., Ogata T., Maruyama T., Kamiya H. (2004) Octocoral chemical signaling selects and controls dinoflagellate symbionts. The Biological Bulletin 207, 80-86.

Krämer W.E., Caamaño-Ricken I., Richter C., Bischof K. (2012) Dynamic regulation of photoprotection determines thermal tolerance of two phylotypes of Symbiodinium clade A at two photon fluence rates. Photochemistry and Photobiology 88, 398-413.

Krieger-Liszkay A. (2005) Singlet oxygen production in photosynthesis. Journal of Experimental Botany 56, 337-346.

Kübler J.E. and Davison I.R. (1993) High-temperature tolerance of photosynthesis in the red alga Chondrus crispus. Marine Biology 117, 327-335.

LaJeunesse T.C. (2001) Investigating the biodiversity, ecology, and phylogeny of endosymbiotic dinoflagellates in the genus Symbiodinium using the ITS region: In search of a "species" level marker. Journal of Phycology 37, 866-880.

LaJeunesse T.C. (2002) Diversity and community structure of symbiotic dinoflagellates from Caribbean coral reefs. Marine Biology 141, 387-400. 
LaJeunesse T.C. (2005) "Species” radiations of symbiotic dinoflagellates in the Atlantic and Indo-Pacific since the miocene-pliocene transition. Molecular Biology and Evolution $22(3), 570-581$.

LaJeunesse T.C. and Thornhill D.J. (2011) Improved resolution of reef-coral endosymbiont (Symbiodinium) species diversity, ecology, and evolution through psbA non-coding region genotyping. PLoS ONE 6(12), e29013.

LaJeunesse T.C., Bhagooli R., Hidaka M., deVantier L., Done T., Schmidt G.W., Fitt W.K., Hoegh-Guldberg O. (2004) Closely related Symbiodinium spp. differ in relative dominance in coral reef host communities across environmental, latitudinal and biogeographic gradients. Marine Ecology Progress Series 284, 147-161.

LaJeunesse T.C., Loh W.K.W., Van Woesik R., Hoegh-Guldberg O., Schmidt G.W., Fitt W.K. (2003) Low symbiont diversity in southern Great Barrier Reef corals, relative to those of the Caribbean. Limnology and Oceanography 48(5), 2046-2054.

LaJeunesse T.C., Pettay D.T., Sampayo E.M., Phongsuwan N., Brown B., Obura D.O., Hoegh-Guldberg O., Fitt W.K. (2010) Long-standing environmental conditions, geographic isolation and host-symbiont specificity influence the relative ecological dominance and genetic diversification of coral endosymbionts in the genus Symbiodinium. Journal of Biogeography 37, 785-800.

LaJeunesse T.C., Reyes Bonilla H., Warner M.E., Wills M., Schmidt G.W., Fitt W.K. (2008) Specificity and stability in high latitude eastern Pacific coral-algal symbioses. Limnology and Oceanography 53(2), 719-727.

Lakeman M.B., von Dassow P., Cattolico R.A. (2009) The strain concept in phytoplankton ecology. Harmful Algae 8, 746-758.

Lanza G.R. and Cairns, J. Jr (1972) Physio-morphological effects of abrupt thermal stress on diatoms. Transactions of the American Microscopical Society 91(3), 276-298. 
References

Ledford H.K. and Niyogi K.K. (2005) Singlet oxygen and photo-oxidative stress management in plants and algae. Plant, Cell and Environment 28, 1037-1045.

Leggat W., Hoegh-Guldberg O., Dove S., Yellowlees D. (2007) Analysis of an EST library from the Dinoflagellate (Symbiodinium sp.) symbiont of reef-building corals. Journal of Phycology 43, 1010-1021.

Leggat W., Seneca F., Wasmund K., Ukani L., Yellowlees D., Ainsworth T.D. (2011) Differential responses of the coral host and their algal symbiont to thermal stress. PLoS ONE 6(10), e26687.

Leisinger U., Rüfenacht K., Fischer B., Pesaro M., Spengler A., Zehnder A.J.B., Eggen R.I.L. (2001) The glutathione peroxidase homologous gene from Chlamydomonas reinhardtii is transcriptionally up-regulated by singlet oxygen. Plant Molecular Biology 46, 395-408.

Lesser M.P. (1996) Elevated temperatures and ultraviolet radiation cause oxidative stress and inhibit photosynthesis in symbiotic dinoflagellates. Limnology and Oceanography 41(2), 271-283.

Lesser M.P. (1997) Oxidative stress causes coral bleaching during exposure to elevated temperatures. Coral Reefs 16, 187-192.

Lesser M.P. (2006) Oxidative stress in marine environments: Biochemistry and Physiological Ecology. Annual Review of Physiology 68, 253-78.

Lesser M.P. (2007) Coral reef bleaching and global climate change: Can corals survive the next century? Proceedings of the National Academy of Sciences 104, 5259-5260.

Lesser M.P. (2011). Coral bleaching: Causes and Mechanisms. In Z. Dubinsky and N. Stambler (eds.) Coral Reefs: An Ecosystem in transition, Springer, 405-419. 
Lesser M.P. (2012) Oxidative stress in tropical marine ecosystems. In D. Abele, J.P. Vázquez-Medina, T. Zenteno-Savín (eds.) Oxidative Stress in Aquatic Ecosystems, Blackwell Publishing Ltd.

Lesser M.P. and Farrell J.H. (2004) Exposure to solar radiation increases damage to both host tissues and algal symbionts of corals during thermal stress. Coral Reefs 23, 367-377.

Lesser M.P. and Shick J.M. (1989a) Effects of irradiance and ultraviolet radiation on photoadaptation in the zooxanthellae of Aiptasia pallida: primary production, photoinhibition, and enzymic defenses against oxygen toxicity. Marine Biology 102, 243255.

Lesser M.P. and Shick J.M. (1989b) Photoadaption and defenses against oxygen toxicity in zooxanthellae from natural populations of symbiotic cnidarians. Journal of Experimental Marine Biology and Ecology 134, 129-141.

Lesser M.P., Stochaj W.R., Tapley D.W., Shick J.M. (1990) Bleaching in coral reef anthozoans: effects of irradiance, ultraviolet radiation, and temperature on the activities of protective enzymes against active oxygen. Coral Reefs 8, 225-232.

Levy O., Achituv Y., Yacobi Y.Z., Dubinsky Z., Stambler N. (2006b) Diel 'tuning' of coral metabolism: physiological responses to light cues. The Journal of Experimental Biology 209, 273-283.

Levy O., Achituv Y., Yacobi Y.Z., Stambler N., Dubinsky Z. (2006b) The impact of spectral composition and light periodicity on the activity of two antioxidant enzymes (SOD and CAT) in the coral Favia favus. Journal of Experimental Marine Biology and Ecology $328,35-46$.

Liñán-Cabello M.A., Flores-Ramirez L.A., Zenteno-Savin T., Olguin- Monroy N.O., Sosa-Avalos R., Patiño-Barragan M., Olivos-Ortiz A. (2009) Seasonal changes of antioxidant and oxidative parameters in the coral Pocillopora capitata on the Pacific coast of Mexico. Marine Ecology 31, 407-417. 
Littman R.A., Bourne D.G., Willis B.L. (2010) Responses of coral-associated bacterial communities to heat stress differ with Symbiodinium type on the same coral host. Molecular Ecology 19, 1978-1990.

Logan D.D.K., LaFlamme A.C., Weis V.M., Davy S.K. (2010) Flow-cytometric characterization of the cell-surface glycans of symbiotic dinoflagellates (Symbiodinium spp.). Journal of Phycology 46, 525-533.

Lough J.M. and Van Oppen M.J.H. (2009) Introduction: coral bleaching - Patterns, processes, causes and consequences. In M.J.H. van Oppen, J.M. Lough (eds.) Coral Bleaching. Springer-Verlag Berlin Heidelberg, 1-5.

Loya Y., Sakai K., Yamazato K., Nakano Y., Sambali H., Van Woesik R. (2001) Coral bleaching: the winners and the losers. Ecology Letters 4, 122-131.

Makino A., Miyake C., Yokota A. (2002) Physiological functions of the water-water cycle (Mehler reaction) and the cyclic electron flow around PSI in rice leaves. Plant and Cell Physiology 43(9), 1017-1026.

Manasrah R., Raheed M., Badran M.I. (2006) Relationships between water temperature, nutrients and dissolved oxygen in the northern Gulf of Aqaba, Red Sea. Oceanologia 48 (2), 237-253.

Marutani Y., Yamauchi Y., Kimura Y., Mizutani M., Sugimoto Y. (2012) Damage to photosystem II due to heat stress without light-driven electron flow: involvement of enhanced introduction of reducing power into thylakoid membranes. Planta, DOI: 10.1007/s00425-012-1647-5.

Maynard J.A., Anthony K.R.N., Marshall P.A., Masiri I. (2008) Major bleaching events can lead to increased thermal tolerance in corals. Marine Biology 155, 173-182. 
McCabe Reynolds J., Bruns B.U., Fitt W.K., Schmidt G.W. (2009) Enhanced photoprotection pathways in symbiotic dinoflagellates of shallow-water corals and other cnidarians. Proceedings of the National Academy of Sciences 105(36), 13674-13678.

McGinty E.S., Pieczonka J., Mydlarz L.D. (2012) Variations in reactive oxygen release and antioxidant activity in multiple Symbiodinium types in response to elevated temperature. Microbial Ecology 64(4), 1000-1007.

Merle P.L., Sabourault C., Richier S., Allemand D., Furla P. (2007) Catalase characterization and implication in bleaching of a symbiotic sea anemone. Free Radical Biology \& Medicine 42, 236-246.

Middlebrook R., Hoegh-Guldberg O., Leggat W. (2008) The effect of thermal history on the susceptibility of reef-building corals to thermal stress. The Journal of Experimental Biology 211, 1050-1056.

Mieog J.C., Olsen J.L., Berkelmans R., Bleuler-Martinez S.A., Willis B.L., Van Oppen M.J.H. (2009) The roles and interactions of symbiont, host and environment in defining coral fitness. PLoS ONE 4(7), 1-12.

Mittler R. (2002) Oxidative stress, antioxidants and stress tolerance. TRENDS in Plant Science 7, 405-410.

Mittler R., Vanderauwera S., Suzuki N., Miller G., Tognetti V.B., Vandepoele K., Gollery M., Shulaev V., Van Breusegem F. (2011) ROS signaling: the new wave? Trends in Plant Science 16(6), 300-309.

Mobley K.B. and Gleason D.F. (2003) The effect of light and heterotrophy on carotenoid concentrations in the Caribbean anemone Aiptasia pallida (Verrill). Marine Biology 143, $629-637$. 
Mostafavi P.G., Fatem S.M.R., Shahhosseiny M.H., Hoegh-Guldberg O., Loh W.K.W. (2007) Predominance of clade D Symbiodinium in shallow-water reef-building corals off Kish and Larak Islands (Persian Gulf, Iran). Marine Biology 153, 25-34.

Müller P., Li X.-P., Niyogi K.K. (2001) Non-photochemical quenching. A response to excess light energy. Plant Physiology 125, 1558-1566.

Muller-Parker G. and Davy S.K. (2001) Temperate and tropical algal-sea anemone symbioses. Invertebrate Biology 120, 104-123.

Muller-Parker G., Pierce-Cravens J., Bingham B.L. (2007) Broad thermal tolerance of the symbiotic dinoflagellate Symbiodininum muscatinei (Dinophyta) in the sea anemone Anthopleura elegantissima (Cnidaria) from northern latitudes. Journal of Phycology 43, $25-31$.

Mullineaux P., Ball L., Escobar C., Karpinska B., Creissen G., Karpinski S. (2000) Are diverse signalling pathways integrated in the regulation of Arabidopsis antioxidant defence gene expression in response to excess excitation energy? Philosophical Transactions of the Royal Society B 355, 1531-1540.

Murata N., Takahashi S., Nishiyama Y., Allakhverdiev S.I. (2007) Photoinhibition of photosystem II under environmental stress. Biochimica et Biophysica Acta 1767, 414-421.

Muscatine L., Falkowski P.G., Porter J.W., Dubinsky Z. (1984) Fate of photosynthetic fixed carbon in light- and shade-adapted colonies of the symbiotic coral Stylophora pistillata. Proceedings of the Royal Society B 222(1227), 181-202.

Muscatine L., Ferrier-Pagès C., Blackburn A., Gates R.D., Baghdasarian G., Allemand D. (1998) Cell-specific density of symbiotic dinoflagellates in tropical anthozoans. Coral Reefs 17, 329-337.

Nakano Y. and Asado K. (1981) Hydrogen peroxide is scavenged by ascorbate-specific peroxidase in spinach chloroplasts. Plant and Cell Physiology 22(5):867-880. 
Nii C.M and Muscatine L. (1997) Oxidative stress in the symbiotic sea anemone Aiptasia pulchella (Carlgren, 1943): Contribution of the animal to superoxide ion production at elevated temperature. The Biological Bulletin 192, 444-456.

Nishiyama Y., Allakhverdiev S.I., Murata N. (2006) A new paradigm for the action of reactive oxygen species in the photoinhibition of photosystem II. Biochimica et Biophysica Acta 1757, 742-749.

Niyogi K.K. (1999) Photoprotection revisited: Genetic and molecular approaches. Annual Review of Plant Physiology and Plant Molecular Biology 50, 333-59.

NOAA - National Oceanic and Atmospheric Administration:

http://www.ncdc.noaa.gov/oa/climate/research/sst/sst.php

NOAA (2010) Heat Stress to Caribbean Corals in 2005 Worst on Record. http://www.noaanews.noaa.gov/stories2010/20101115_coralbleaching.html.

Norström A.V., Nyström M., Lokrantz J., Folke C. (2009) Alternative states on coral reefs: beyond coral-macroalgal phase shifts. Marine Ecology Progress Series 376, 295-306.

Obura D.O. (2008) Reef corals bleach to resist stress. Marine Pollution Bulletin 58(2), 206-212.

Okamoto O.K., Robertson D.L., Fagan T.F., Woodland Hastings J., Colepicolo P. (2001) Different regulatory mechanisms modulate the expression of a dinoflagellate ironsuperoxide dismutase. The Journal of Biological Chemistry 276, 19989-19993.

Oliver T.A. and Palumbi S.R. (2011) Do fluctuating temperature environments elevate coral thermal tolerance? Coral Reefs 30, 429-440. 
Op den Camp R.G.L., Przybyla D., Ochsenbein C., Laloi C., Kim C., Danon A., Wagner D., Hideg E., Göbel C., Feussner I., Nater M., Apel K. (2003) Rapid induction of distinct stress responses after the release of singlet oxygen in Arabidopsis. The Plant Cell 15, 2320-2332.

Ou B., Huang D., Hampsch-Woodill M., Flanagan J.A., Deemer E.K. (2002) Analysis of antioxidant activities of common vegetables employing oxygen radical absorbance capacity (ORAC) and ferric reducing antioxidant power (FRAP) assays: A comparative study. Journal of Agricultural and Food Chemistry 50, 3122-3128.

Palmer C.V., Bythell J.C., Willis B.L. (2010) Levels of immunity parameters underpin bleaching and disease susceptibility of reef corals. The FASEB Journal 24, 1935-1946.

Palmer C.V., Modi C.K., Mydlarz L.D. (2009) Coral fluorescent proteins as antioxidants. PLoS ONE 4(10), 1-9.

Perez S. and Weis V. (2006) Nitric oxide and cnidarian bleaching: an eviction notice mediates breakdown of a symbiosis. The Journal of Experimental Biology 209, 2804-2810.

Perez S.F. (2007) Exploring the cellular mechanisms of cnidarian bleaching in the sea anemone Aiptasia pallida. Dissertation, Oregon State University, Oregon, USA.

Perez S.F., Cook C.B., Brooks W.R. (2001). The role of symbiotic dinoflagellates in the temperature induced bleaching response of the subtropical sea anemone Aiptasia pallida. Journal of Experimental Marine Biology and Ecology 256, 1-14.

Perry C.T. and Larcombe P. (2003) Marginal and non-reef-building coral environments. Coral Reefs 22, 427-432.

Plantivaux A., Furla P., Zoccola D., Garello G., Forcioli D., Richier S., Merle P.-L., Tambutté E., Tambutté S. and Allemand D. (2004) Molecular characterization of two CuZn-superoxide dismutases in a sea anemone. Free Radical Biology \& Medicine 37(8), $1170-1181$. 
Platt, T., Gallegos, C.L., Harrison, W.G. (1980) Photoinhibition of photosynthesis in natural assemblages of marine phytoplankton. Journal of Marine Research 38, 687-701.

Pochon X. and Gates R.D. (2010) A new Symbiodinium clade (Dinophyceae) from soritid foraminifera in Hawai'i. Molecular Phylogenetics and Evolution 56, $492-497$.

Prior R. L., Wu X. and Schaich K. (2005). Standardized methods for the determination of antioxidant capacity and phenolics in foods and dietary supplements. Journal of Agricultural and Food Chemistry 53, 4290-4302.

Pulido R., Bravo L., Saura-Calixto F. (2000) Antioxidant activity of dietary polyphenols as determined by a modified ferric reducing/antioxidant power assay. Journal of Agricultural and Food Chemistry 48, 3396-3402.

Putnam H.M. and Edmunds P.J. (2010) The physiological response of reef corals to diel fluctuations in seawater temperature. Journal of Experimental Marine Biology and Ecology 396, 216-222.

Ragni M., Airs R.L., Hennige S.J., Suggett D.J., Warner M.E., Geider R.J. (2010) PSII photoinhibition and photorepair in Symbiodinium (Pyrrhophyta) differs between thermally tolerant and sensitive phylotypes. Marine Ecology Progress Series 406, 57-70.

Ralph P.J., Gademann R., Larkum A.W.D. (2001) Zooxanthellae expelled from bleached corals at $33{ }^{\circ} \mathrm{C}$ are photosynthetically competent. Marine Ecology Progress Series 220, 163-168.

Ralph P.J., Gademann R., Larkum A.W.D., Kühl M. (2002) Spatial heterogeneity in active chlorophyll fluorescence and PSII activity of coral tissues. Marine Biology 141, 639-646. 
Richier S., Cottalorda J.-M., Guillaume M.M.M., Fernandez C., Allemand D., Furla P. (2008) Depth-dependant response to light of the reef building coral, Pocillopora verrucosa: Implication of oxidative stress. Journal of Experimental Marine Biology and Ecology 357, 48-56.

Richier S., Furla P., Plantivaux A., Merle P-L., Allemand D. (2005) Symbiosis-induced adaptation to oxidative stress. The Journal of Experimental Biology 208, 277-285.

Richier S., Merle P.-L., Furla P., Pigozzia D., Sola F., Allemand D. (2003) Characterization of superoxide dismutases in anoxia- and hyperoxia-tolerant symbiotic cnidarians. Biochimica et Biophysica Acta 1621, 84- 91.

Richier S., Sabourault C., Courtiade J., Zucchini N., Allemand D., Furla P. (2006) Oxidative stress and apoptotic events during thermal stress in the symbiotic sea anemone, Anemonia viridis. FEBS Journal 273, 4186-4198.

Riegl B. and Piller W.E. (2003) Possible refugia for reefs in times of environmental stress. International Journal of Earth Sciences / Geologische Rundschau 92, 520-531.

Robison J.D. and Warner M.E. (2006) Differential impacts of photoacclimation and thermal stress on the photobiology of four different phylotypes of Symbiodinium (Pyrrhophyta). Journal of Phycology 42, 568-579.

Rodriguez-Lanetty M., Loh W., Carter D., Hoegh-Guldberg O. (2001) Latitudinal variability in symbiont specificity within the widespread scleractinian coral Plesiastrea versipora. Marine Biology 138, 1175-1181.

\section{Rodriguez-Lanetty M., Wood-Charlson E.M., Hollingsworth L.L., Krupp D.A.,} Weis V.M. (2006) Temporal and spatial infection dynamics indicate recognition events in the early hours of a dinoflagellate/coral symbiosis. Marine Biology 149, 713-719.

Rosenberg E. and Falkovitz L. (2004) The Vibrio shiloilOculina patagonica model system of coral bleaching. Annual Review of Microbiology 58, 143-59. 
Rosic N.N., Pernice M., Dove S., Dunn S., Hoegh-Guldberg O. (2010) Gene expression profiles of cytosolic heat shock proteins Hsp70 and Hsp90 from symbiotic dinoflagellates in response to thermal stress: possible implications for coral bleaching. Cell Stress and Chaperones 16, 69-80.

Rowan R. (1998) Diversity and ecology of zooxanthellae on coral reefs. Journal of Phycology 34, 407-417.

Rowan R. (2004) Thermal adaptation in reef coral symbionts. Nature 430. 742.

Rowan R. and Knowlton N. (1995) Intraspecific diversity and ecological zonation in coral-algal symbiosis. Proceedings of the National Academy of Sciences 92, 2850-2853.

Rowan R. and Powers D.A. (1992) Ribosomal RNA sequences and the diversity of symbiotic dinoflagellates (zooxanthellae). Proceedings of the National Academy of Sciences 89, 3639-3643.

Rowan R., Knowlton N., Baker A., Jara J. (1997) Landscape ecology of algal symbionts creates variation in episodes of coral bleaching. Nature 388, 265-269.

Salih A., Larkum A., Cox G., Kühl M., Ove Hoegh-Guldberg O. (2000) Fluorescent pigments in corals are photoprotective. Nature 408, 850-853.

Sampayo E.M., Franceschinis L., Hoegh-Guldberg O., Dove S. (2007) Niche partitioning of closely related symbiotic dinoflagellates. Molecular Ecology 16, 3721-3733.

Sampayo E.M., Ridgway T., Bongaerts P., Hoegh-Guldberg O. (2008) Bleaching susceptibility and mortality of corals are determined by fine-scale differences in symbiont type. Proceedings of the National Academy of Sciences 105(30), 1044-10449. 
Santos S.R., Shearer T.L., Hannes A.R., Coffroth M.A. (2004) Fine-scale diversity and specificity in the most prevalent lineage of symbiotic dinoflagellates (Symbiodinium Dinophyceae) of the Caribbean. Molecular Ecology 13, 459-469.

Santos S.R., Taylor D.J., Coffroth M.A. (2001) Genetic comparisons of freshly isolated versus cultured symbiotic dinoflagellates: Implications for extrapolating to the intact symbiosis. Journal of Phycology 37, 900-912.

Saragosti E., Tchernov D., Katsir A., Shaked Y. (2010) Extracellular production and degradation of superoxide in the coral Stylophora pistillata and cultured Symbiodinium. Public library of Science One 5, 1-10.

Savage A.M., Trapido-Rosenthal H., Douglas A.E. (2002) On the functional significance of molecular variation in Symbiodinium, the symbiotic algae of Cnidaria: photosynthetic response to irradiance. Marine Ecology Progress Series 244, 27-37.

Schoenberg D.A. and Trench R.K. (1980a) Genetic variation in Symbiodinium (=Gymnodinium) microadriaticum Freudenthal, and specificity in its symbiosis with marine invertebrates. I. Isoenzyme and soluble protein patterns of axenic cultures of Symbiodinium microadriaticumt. Proceedings of the Royal Society B 207, 405-427.

Schoenberg D.A. and Trench R.K. (1980b) Genetic variation in Symbiodinium (=Gymnodinium) microadriaticum Freudenthal, and specificity in its symbiosis with marine invertebrates III Specificity and Infectivity of Symbiodinium microadraticum. Proceedings of the Royal Society B 207, 445-460.

Schwarz J.A., Krupp D.A., Weis V.M. (1999) Late larval development and onset of symbiosis in the scleractinian coral Furtgia scutaria. The Biological Bulletin 196, 70-79.

Shick J.M. (2004) The continuity and intensity of ultraviolet irradiation affect the kinetics of biosynthesis, accumulation, and conversion of mycosporine-like amino acids (MAAs) in the coral Stylophora pistillata. Limnology and Oceanography 49(2), 442-458. 
Shick J.M. and Dunlap W.C. (2002) Mycosporine-like amino acids and related gadusols: Biosynthesis, accumulation, and UV-protective functions in aquatic organisms. Annual Review of Physiology 64, 223-62.

Shinzato C., Shoguchi E., Kawashima T., Hamada M., Hisata K., Tanaka M., Fujie M., Fujiwara M., Koyanagi R., Ikuta T., Fujiyama A., Miller D.J., Satoh N. (2011) Using the Acropora digitifera genome to understand coral responses to environmental change. Nature 476, 320-324.

Sies H. (1997) Impaired endothelial and smooth muscle cell function in oxidative stress: Oxidants and antioxidants. Experimental Physiology 82, 291-295.

Sigaud-Kutner T.C.S., Pinto E., Okamoto O.K., Latorre L.R., Colepicolo P. (2002) Changes in superoxide dismutase activity and photosynthetic pigment content during growth of marine phytoplankters in batch-cultures. Plant Physiology 114, 566-571.

Smith D.J., Suggett D.J., Baker N.R. (2005) Is photoinhibition of zooxanthellae photosynthesis the primary cause of thermal bleaching in corals? Global Change Biology $11,1-11$.

Smith-Keune C. and Van Oppen M. (2006) Genetic structure of a reef-building coral from thermally distinct environments on the Great Barrier Reef. Coral Reefs 25, 493-502.

Soh N. (2006) Recent advances in fluorescent probes for the detection of reactive oxygen species. Analytical and Bioanalytical Chemistry 386, 532-543.

Stambler N. and Dubinsky Z. (2004) Stress effects on metabolism and photosynthesis of hermatypic corals. In E. Rosenberg and Y. Loya (eds.) Coral health and disease, Heidelberg New York, Springer Verlag, 195-215. 
Starcevic A., Dunlap W.C., Cullum J., Shick J.M., Hranueli D. and Long P.F. (2010)

Gene expression in the scleractinian Acropora microphthalma exposed to high solar irradiance reveals elements of photoprotection and coral bleaching. PLoS ONE 5(11), e13975.

Stat M., Carter D., Hoegh-Guldberg O. (2006) The evolutionary history of Symbiodinium and scleractinian hosts-Symbiosis, diversity, and the effect of climate change. Perspectives in Plant Ecology, Evolution and Systematics 8, 23-43.

Stat M., Pochon X., Cowie R.O.M., Gates R.D. (2009). Specificity in communities of Symbiodinium in corals from Johnston Atoll. Marine Ecology Progress Series 386, 83-96.

Steinke M., Brading P., Kerrison P., Warner M.E., Suggett D.J. (2011) Concentrations of dimethylsufoniopropionate and dimethyl sulfide are strain-specific in symbiotic dinoflagellates (Symbiodinium sp., Dinophyceae). Journal of Phycology 47, 775-783.

Stimson J., and Kinzie III R.A. (1991). The temporal pattern and rate of release of zooxanthellae from reef coral Pocillopora damicornis (Linnaeus) under nitrogenenrichment and control conditions. Journal of Experimental Marine Biology and Ecology $153,63-74$.

Strychar K.B., Sammarco P.W., Piva T.J. (2004) Apoptotic and necrotic stages of Symbiodinium (Dinophyceae) cell death activity: bleaching of soft and scleractinian corals. Phycologia 43(6), 768-777.

Suggett D.J., Warner M.E., Smith D.J., Davey P., Hennige S., Baker N.R. (2008) Photosynthesis and production of hydrogen peroxide by Symbiodinium (Pyrrhophyta) phylotypes with different thermal tolerances. The Journal of Phycology 44, 948-956.

Sunda W., Kieber D.J., Kiene R.P., Huntsman S. (2002) An antioxidant function for DMSP and DMS in marine algae. Nature 418, 317-320.

Surf-forecast.com: www.surf-forecast.com/breaks/Oku/seatemp 
Szmant-Froelich A., Reutter M., Riggs L. (1985) Sexual reproduction of Favia fragum (Esper): Lunar patterns of gametogenesis, embryogenesis and planulation in Puerto Rico. Bulletin of Marine Science 37(3), 880-892.

Takahashi S. and Murata N. (2008) How do environmental stresses accelerate photoinhibition? Trends in Plant Science 13(4), 178-182.

Takahashi S., Whitney S., Itoh S., Maruyama T. , Badger M. (2008) Heat stress causes inhibition of the de novo synthesis of antenna proteins and photobleaching in cultured Symbiodinium. Proceedings of the National Academy of Sciences 105, 4203-4208.

Tchernov D., Gorbunov M.Y., de Vargas C., Yadav S.N., Milligan A.J., Häggblom M., Falkowski P.G. (2004) Membrane lipids of symbiotic algae are diagnostic of sensitivity to thermal bleaching in corals. Proceedings of the National Academy of Sciences 101, 13531-13535.

Tchernov D., Kvitt H., Haramaty L., Bibby T.S., Gorbunov M.Y., Rosenfeld H., Falkowski P.G. (2011) Apoptosis and the selective survival of host animals following thermal bleaching in zooxanthellate corals. Proceedings of the National Academy of Sciences 108(24), 9905-9909.

The National Center for Biotechnology Information (NCBI): http://www.ncbi.nlm.nih.gov/

Thornhill D.J., LaJeunesse T.C., Santos S.R. (2007) Measuring rDNA diversity in eukaryotic microbial systems: how intragenomic variation, pseudogenes, and PCR artifacts confound biodiversity estimates. Molecular Ecology 16, 5326-5340.

Titlyanov E.A., Titlyanova T.V., Leletkin V.A., Tsukahara J., van Woesik R., Yamazato K. (1996) Degradation of zooxanthellae and regulation of their density in hermatypic corals. Marine Ecology Progress Series 139, 167-178. 
Toller W.W., Rowan R., Knowlton N. (2001) Zooxanthellae of the Montastraea annularis species complex: Patterns of distribution of four taxa of Symbiodinium on different reefs and across depths. The Biological Bulletin 201, 348-359.

Trapido-Rosenthal H., Zielke S., Owen R., Buxton L., Boeing B., Bhagooli R., Archer J. (2005) Increased zooxanthellae nitric oxide synthase activity is associated with coral bleaching. The Biological Bulletin 208, 3-6.

Trapido-Rosenthal H.G., Sharp K.H., Galloway T.S., Morrall C.E. (2001) Nitric oxide and cnidarian-dinoflagellate symbioses: Pieces of a puzzle. American Zoologist 41, 247257.

Trench R.K. and Blank R.J. (1987) Symbiodinium microadraticum Freudenthal, S. goreauii sp. Nov., S. kawagutii sp. Nov. and S. pilosum sp. Nov.: Gymnodinioid dinoflagellate symbionts of marine invertebrates. Journal of Phycology 23, 469-481.

Ulstrup K.E., Hill R., Van Oppen M.J.H., Larkum A.W.D. and Ralph P.J. (2008) Seasonal variation in the photo-physiology of homogeneous and heterogeneous Symbiodinium consortia in two scleractinian corals. Marine Ecology Progress Series 361, 139-150.

Ulstrup K.E., Ralph P.J., Larkum A.W.D. and Kühl M. (2006) Intra-colonial variability in light acclimation of zooxanthellae in coral tissues of Pocillopora damicornis. Marine Biology 149, 1325-1335.

Van Oppen M.J.H. (2004) Mode of zooxanthella transmission does not affect zooxanthella diversity in acroporid corals. Marine Biology 144, 1-7.

Van Oppen M.J.H. and Gates R.D. (2006) Conservation genetics and the resilience of reef-building corals. Molecular Ecology 15, 3863-3883. 
Van Oppen M.J.H., Bongaerts P., Underwood J.N., Peplow L.M. and Cooper T.F. (2011) The role of deep reefs in shallow reef recovery: an assessment of vertical connectivity in a brooding coral from west and east Australia. Molecular Ecology 20, $1647-1660$.

Veljovic -Jovanovic S. (1998) Active oxygen species and photosynthesis: Mehler and ascorbate peroxidase reactions. Yugoslav Physiol. Pharmacol. Acta 34, 503-522.

Venn A. A., Loram J. E., Douglas A. E. (2008) Photosynthetic symbioses in animals. The Journal of Experimental Botany 59, 1069-1080.

Venn A.A., Tambutté E., Lotto S., Zoccola D., Allemand D., Tambutté S. (2009) Imaging intracellular $\mathrm{pH}$ in a reef coral and symbiotic anemone. Proceedings of the National Academy of Sciences 106(39), 16574-16579.

Visram S. and Douglas A.E. (2007) Resilience and acclimation to bleaching stressors in the scleractinian coral Porites cylindrica. Journal of Experimental Marine Biology and Ecology 349, 35-44.

Wagner D., Przybyla D., Op den Camp R., Kim C., Landgraf F., Lee K.P., Würsch M., Laloi C., Nater M., Hideg E., Apel K. (2005) The genetic basis of singlet oxygeninduced stress responses in Arabidopsis thaliana. Science 306, 1183-1185.

Wakefield T.S., Farmer M.A., Kempf S.C. (2000) Revised description of the fine structure of in situ "zooxanthellae" genus Symbiodinium. The Biological Bulletin 199, 76-84.

Wang J., Zhu J., Liu S., Liu B., Gao Y., Wua Z. (2011) Generation of reactive oxygen species in cyanobacteria and green algae induced by allelochemicals of submerged macrophytes. Chemosphere 85, 977-982. 
References

Wang J.-T. and Douglas A.E. (1997) Nutrients, signals, and photosynthate release by symbiotic algae' The impact of taurine on the dinoflagellate alga Symbiodinium from the sea anemone Aiptasia pulchella. Plant Physiology 114, 631-636.

Wang J.-T., Meng P.-J., Chen Y.-Y., Chen C.A. (2012) Determination of the thermal tolerance of Symbiodinium using the activation energy for inhibiting photosystem II activity. Zoological Studies 51(2), 137-142.

Warner M.E., Chilcoat G.C., McFarland F.K., Fitt W.K. (2002) Seasonal fluctuations in the photosynthetic capacity of photosystem II in symbiotic dinoflagellates in the Caribbean reef-building coral Montastraea. Marine Biology 141, 31-38.

Warner M.E., Fitt W.K., Schmidt G.W. (1999) Damage to photosystem II in symbiotic dinoflagellates: A determinant of coral bleaching. Proceedings of the National Academy of Sciences 96, 8007-8012.

Warner M.E., LaJeunesse T.C., Robison J.D., Thur R.M. (2006) The ecological distribution and comparative photobiology of symbiotic dinoflagellates from reef corals in Belize: Potential implications for coral bleaching. Limnology and Oceanography 51(4), 1887-1897.

Weis V.M. (2008). Cellular mechanisms of Cnidarian bleaching: stress causes the collapse of symbiosis. The Journal of Experimental Biology 211, 3059-3066.

Weis V.M. (2010) The susceptibility and resilience of corals to thermal stress: adaptation, acclimatization or both? Molecular Ecology 19, 1515-1517.

Weis V.M., Davy S.K., Hoegh-Guldberg O., Rodriguez-Lanetty M., Pringle J.R. (2008) Cell biology in model systems as the key to understanding corals. Trends in Ecology \& Evolution 23, 369-376. 
Weis V.M., Reynolds W.S., DeBoer M.D., Krupp D.A. (2000) Host-symbiont specificity during onset of symbiosis between the dinoflagellates Symbiodinium spp. and planula larvae of the sleractinian coral Fungia scutaria. Coral Reefs 20, 301-308.

Wicks L.C. (2009) Persistence of corals in marginal habitats: the role of the environment, and symbiont diversity and ecophysiology. Dissertation. Victoria University of Wellington, New Zealand.

Wicks L.C., Hill R., Davy S.K. (2010) The influence of irradiance on tolerance to high and low temperature stress exhibited by Symbiodinium in the coral, Pocillopora damicornis, from the high-latitude reef of Lord Howe Island. Limnology and Oceanography 55(6), 2476-2486.

Wilkerson F.P., Kobayashi D., Muscatine L. (1988) Mitotic index and size of symbiotic algae in Caribbean Reef corals. Coral Reefs 7, 29-36.

Wolfe K.L. and Liu R.H. (2007) Cellular Antioxidant Activity (CAA) assay for assessing antioxidants, foods, and dietary supplements. Journal of Agricultural and Food Chemistry $55,8896-8907$.

Wood-Charlson E.M., Hollingsworth L.L., Krupp D.A., Weis V.M. (2006) Lectin/glycan interactions play a role in recognition in a coral/dinoflagellate symbiosis. Cellular Microbiology 8(12), 1985-1993.

Wrona M. and Wardman P. (2006) Properties of the radical intermediate obtained on oxidation of 2',7'-dichlorodihydrofluorescein, a probe for oxidative stress. Free Radical Biology \& Medicine 41, 657-667.

WWF: The importance of coral to people, http://www.worldwildlife.org/what/wherewework/coraltriangle/importance-of-coral.html. 
References

Yakovleva I., Bhagooli R., Takemurac A., Hidaka M. (2004) Differential susceptibility to oxidative stress of two scleractinian corals: antioxidant functioning of mycosporineglycine. Comparative Biochemistry and Physiology, Part B 139, 721-730.

Yellowlees D., Rees T.A.V., Leggat W. (2008) Metabolic interactions between algal symbionts and invertebrate hosts. Plant, Cell and Environment 31, 679-694.

Yuan J. and Shiller A.M. (1999) Determination of subnanomolar levels of hydrogen peroxide in seawater by reagent-injection chemiluminescence detection. Analytical Chemistry 71, 1975-1980. 


\section{Appendix}

\section{Appendix A - Molecular Typing of Symbiodinium}

\section{A.1 - Laboratory Symbiodinium types}

Table A1: complete list of used Symbiodinium cultures with corresponding sub-clade, host, geographic origin, Genbank number and sequence length.

\begin{tabular}{|c|c|c|c|c|c|}
\hline Culture ID & Sub- clade & Host & $\begin{array}{l}\text { Geographic } \\
\text { origin }\end{array}$ & $\begin{array}{l}\text { GenBank no } \\
\text { (\% } \\
\text { agreement) }\end{array}$ & $\begin{array}{l}\text { Sequence } \\
\text { length }\end{array}$ \\
\hline CCMP2467 & A1 & Stylophora pistillata & $\begin{array}{l}\text { Gulf of } \\
\text { Aqaba }\end{array}$ & $\begin{array}{l}\text { AF333505 } \\
(100 \%)\end{array}$ & 255 \\
\hline Zs & $A^{*}$ & Zoanthus sociatus & Jamaica & & \\
\hline S.bermudense & $\begin{array}{l}\text { B1 } \\
\text { (S.bermudense) }\end{array}$ & Aiptasia pallida? & Bermuda & $\begin{array}{l}\text { AF060891 } \\
(99.7 \%)\end{array}$ & 279 \\
\hline Ap1 & $\mathrm{B} 1-\mathrm{O}$ & Aiptasia pulchella & Okinawa & $\begin{array}{l}\text { AF333511 } \\
(100 \%)\end{array}$ & 271 \\
\hline CCMP2459 & B2 & Oculina diffusa & Bermuda & AF333513 & 275 \\
\hline Ap2 & D5 & Unknown anemone & Okinawa & JN558078 & 271 \\
\hline S.californium & $\begin{array}{l}\text { E1 } \\
\text { (S.californium) }\end{array}$ & $\begin{array}{l}\text { Anthopleura } \\
\text { elegantissima }\end{array}$ & $\begin{array}{l}\text { Southern } \\
\text { California }\end{array}$ & $\begin{array}{l}\text { AF334659 } \\
(100 \%)\end{array}$ & 278 \\
\hline A001 & E1-O & Acropora sp. & Okinawa & JN558086 & 278 \\
\hline $\mathrm{Mv}$ & $\mathrm{F} 1-\mathrm{H}$ & $\begin{array}{l}\text { Montipora } \\
\text { verrucosa }\end{array}$ & Hawaii & JN558068 & 288 \\
\hline AiptasiaI & F1-P & Aiptasia pulchella & $\begin{array}{l}\text { Pacific } \\
\text { (location } \\
\text { unknown) }\end{array}$ & JN558068 & 288 \\
\hline $\operatorname{Pd} 45 \mathrm{a}$ & $\mathrm{F} 5.2 \mathrm{e}$ & Porites divaricata & Florida & JN558074 & 285 \\
\hline N/A & A1 & $\begin{array}{l}\text { Anthopleura } \\
\text { aureoradiata }\end{array}$ & $\begin{array}{l}\text { Wellington, } \\
\text { NZ }\end{array}$ & $\begin{array}{l}\text { JN242191 } \\
(98.5 \%)\end{array}$ & 238 \\
\hline N/A & B1 & Aiptasia pulchella & $\begin{array}{l}\text { Pacific } \\
\text { (location } \\
\text { unknown) }\end{array}$ & AF333511 & 251 \\
\hline
\end{tabular}

A*: typed by D. Logan (2006) 
Appendix

Culture sequences: 5.8S ribosomal RNA gene, partial sequence; internal transcribed spacer 2, complete sequence; and 28S ribosomal RNA gene, partial sequence

Symbiodinium type A1:

CTCCGTGAACCAATGGCCTCTTGAACGTGCATTGCGCTCTTGGGATATGCCTGAGAGC ATGTCTGCTTCAGTGCTTCTACTTTCATTTTCTGCTGCTCTTGTTATCAGGAGCAGTGTT GCTGCATGCTTCTGCAAGTGGCACTGGCATGCTAAATATCAAGTTTTGCTTGCTGTTGT GACTGATCAACATCTCATGTCGTTTCAGTTGGCGAAACAAAAGCTCATGTGTGTTCTTA ACACTTCCTAGCATGAAGTCAGACAAGTGAACCCGCCGAATTTAAGCATATAGC

Symbiodinium type $S$. bermudense:

CNACTCCGTGAACCGATGGCCTCCTGAACGCGCATTGCGCTCTCGGGATTTCCTGAGA GCAGGTCTGCTTCAGTGCTTAGCATTATCTACCTGTGCTTGCAAGCAGCATGTATGTCT GCATTGCTGCTTCGCTTTCCAACAAGTCATCGATCGCTTTTGTGTTCGTAAATGGCTTG TTTGCTGCCTGGCCCATGCGCCAAGCTTGAGCGTACTGTTGTTCCAAGCTTTGCTTGCA TCGTGCAGCTCAAGCGCGCAGCTGTCGGGATGCTGATGCATGCCCTTAGCATGAAGTC AGACAAGAGAACCCGCTGAATTTAAGCATATAGAAANCGGAGGAA

Symbiodinium type B2:

GTNGTGAATTACAAACTCCGTGAACCGATGGCCTCCTGAACGCGCATTGCGCTCTCGG GATTTCCTGAGAGCAGGTCTGCTTCAGTGCTTAGCATTATCTACCTGTGCTTGCAAGCA GCATGTCTACACTGCTGCTTTGCTTTCCAACAAGTCATCGATCGCGTTTGTGTTCGTAA ATGGCTTGTTTGCTGCCTGGCCCATGCGCCAAGCTTGAGCGTACTGTTGTTCCAAGCTT AGCTTGCATCGTACAGCTCAAGCGCGCAGCTGTTGGGATGCTGATGCATGCCCTTAGC ATGAAGTCAGACAAGAGAACCCGCTGAATTTAAGCATATAAGAAAGCGGAGGAGG

Symbiodinium type D1:

TGNTGAATTGCCNAACTCCGTGAACCAATGGCCCCCTGAACGCGCATTGCACTCTTGG GACTTCCTGAGAGTATGTTTGCTTCAGTGCTTRTTTTACCTCCTTGCAAGGTTCTGTCGC AACCTTGTGCCCTGGCCAGCCACGGGTTAACTTGCCCATGGCTTGCTGAGTAGTGATCT TTTAGAGCAAGCTCTGGCACGCTGTTGTTTGAGGCAGCCTATATTGAGGCTATTTCAAA TGACGTTGCTACAAGCTTGATGTGTCCTTCTGCGCCGTTGCGCATCCCATAGCATGAAG TCAAACAAGAGAACCCGCTGAATTTAAGCATATAAGAAAGCGGAGGACG 
Appendix

Symbiodinium type E1-O:

TTTGTGAATTGCAAACTCCGTGAACCAATAGCACCCTGAACTCGCATTGCACTCTTGG GACACGCCTGAGAGTATGTCTGCTTCAGTGCTTTTCATATCTTCGCAGTGCGGGCTTCC TGGAGAAGCCTTGAGCCTCTTTGCGCGCTGCTGCATCAGAATTTGCAGCGGCGCGCTG AACACAAACCGGGAGGTAAGCTGGACTGATTTGTCGCGCATCACTGGGCACGTGTGTC CGTTTTGGCCCAATCATGCCAGCCTGCCAAGCAATTGGTGCTCAAATACCAATCTTAG CATGAAGTCAGACAAGCAAACCCGCTGAATTTAAGCATATAAGAAGCGGAGGGG

Symbiodinium type F1-H:

CAAACTCCGTGAACCAATGGCCTCCTGAACGTACGTTGCACTCTTGGGATTTCCTGAG AGTATGTCTGCTTCAGTGCTTAGCTTGCCCAATCTTGCGGATAGATTTTGTTTCTGTCTT GCGCCCCTGTGAGCCATTGAAACTCTAGTCAATGGCTTATTGAATGAGTTGGTCTTGCA AAAGCTTTGCGCGATGCTATTCAAGATTCCACCTTGAAATGGTATTTCTTGAGTGACGC TGCTTATGCTTGCAACTGCTGGGATGCTAGCGCATGCCTCTAGCATGAAGTCAGACAA GTGAACCCGCTGAATTTAAGCATA

Symbiodinium type F1-P:

NGAATTGGCGAACTCCGTGAACCAATGGCCTCCTGAACGTNCGTTGCACTCTTGGGAT TTCCTGAGAGTATGTCTGCTTCAGTGCTTAGCTTGCCCAATCTTGCGGATAGATTTTGT TTCTGTCTTGCGCCCCTGTGAGCCATTGAAACTCTAGTCAATGGCTTATTGAATGAGTT GGTCTTGCAAAAGCTTTGCGCGATGCTATTCAAGATTCCACCTTGAAATGGTATTTCTT GAGTGACGCTGCTTATGCTTGCAACTGCTGGGATGCTAGCGCATGCCTCTAGCATGAA GTCAGACAAGTGAACCCGCTGAATTTAAGCATATAAGAAGCGGAGGAACGGANTTCT NCNANNCACA

Symbiodinium type F5.2.e:

TGAATTGCAAACTCCGTGAACCAATGGCCTCCTGAACGTACGTTGCNCTCTTGGGATTT CCTGAGAGTATGTCTGCTTCAGTGCTTAGCTTGCCCAATCTTGCGGACAGATTGTGTTT CTGTCTTGCGCCCCTGTGAGCCATTGAATGTCTACTCAGTGGCTTATTGAATGATTTGG TCTTGCAAAAGCTTTGCGCGCTGCTATTCAAGATTCCACCTTAAAGTGGTATTGCTTGA GTGACGCTGCTTATGCTTGCAGCTGCTGGGATGCTAGCNCAYGCCTCTAGCATGAAGT CAGACAAGCNAACCCGCTGAATTTAAGCATATAAGTAAGCGGAGGAG 
Appendix

Symbiodinium type S. californium:

CTCCTCCGCTTTCTTATATGCTTAAATTCAGCGGGTTTGCTTGTCTGACTTCATGCTAAG ATTGGTATTTGAGCACCAATTGCTTGGCAGGCTGGCATGATTGGGCCAAAACGGACAC ACGTGCCCAGTGATGCGCGACAAATCAGTCCAGCTTACCTCCCGGTTTGTGTTCAGCG CGCCGCTGCAAATTCTGATGCAGCAGCGCACAAAGAGGCTCAAGGCTTCTCCAGGAA GCCCGCACTGCGAAGATATGAAAAGCACTGAAGCAGACATACTCTCAGGCGTGTCCC AAGAGTGCAATGCGAGTTCAGGGTGCTATTGGTTCACGGAGTTTGGCAATTCACAAA

Freshly isolated Symbiodinium type A1-FIS:

AACCAATGGCCTCTTGAACGTGCATTGCGCTCTTGGGATATGCCTGAGAGCATGTCTG CTTCAGTGCTTCTTATATCAATTTTTTTGCTGCTGCTCTTTTTCAAAGGGCAGCGTGGGT GCATGCTACTGCTTGGCAGCACTGGCATGCTTAGATTTGCTGCTTGCTCACTGGTTTGA TTGATCACTTTATCAAATCTTGTCAGTATTGCAAGTCTTGTGTATGTGTTGTGACACAT CCTAGCATGAAGTCAGACAAGCGA

Freshly isolated Symbiodinium type B1-FIS:

GCTCTCGGGATTTCCTGAGAGCAGGTCTGCTTCAGTGCTTAGCATTATCTACCTGTGCT TGCAAGCAGCATGTATGTCTGCATTGCTGCTTCGCTTTCCAACAAGTCATCGATCGCTT TTGTGTTCGTAAATGGCTTGTTTGCTGCCTGGCCCATGCGCCAAGCTTGAGCGTACTGT TGTTCCAAGCTTTGCTTGCATCGTGCAGCTCAAGCGCGCAGCTGTCGGGATGCTGATG CATGCCCTTAGCATGAAGTCAGACAAGAGAACCCGCTGAATTTAAGCATATAAGWAA GCGGAGGATGGTCTTAT 
Appendix

\section{A.2 - Heron Island Symbiodinium types}

Table A.2: Symbiodinium sub-clades present in coral species from Heron Island.

\begin{tabular}{|l|l|l|l|l|}
\hline Host & Sub-clade & $\begin{array}{l}\text { Geneous } \\
(\% \text { agreement })\end{array}$ & GenBank no. & $\begin{array}{l}\text { Sequence } \\
\text { length }\end{array}$ \\
\hline Pocillopora damicornis & $\mathrm{C} 1 / \mathrm{C} 1 \mathrm{c}$ & $100 \% / 99.6 \%$ & EU449103 & 283 \\
\hline Acropora millepora & $\mathrm{C} 3$ & $100 \%$ & AF499789 & 283 \\
\hline Montipora digitata & $\mathrm{C} 15$ & $100 \%$ & AF499789 & 283 \\
\hline Porites cylindrica & $\mathrm{C} 15$ & $100 \%$ & AY239369 & 283 \\
\hline
\end{tabular}

Heron Island sequences: 5.8S ribosomal RNA gene, partial sequence; internal transcribed spacer 2, complete sequence; and 28S ribosomal RNA gene, partial sequence

Symbiodinium type C1 (Pocillopora damicornis):

CAGCGGGTTCACTTGTCTGACTTCATGCTAGAGGCATGCACCTGCATCCCAGCGGTTG CAAGCATGAGCAGCGTCACTCAAGTAAAACCACGAAGGTAGAAACCTGAATAACAGC GCGCAAAGCATTTTGCAAGAACCAAGCAATTCACAAGCCATTGGCTCAGAGGGCAAT AGCTCATAAGAACGCAAGGCAGAAACACATCCTGCTTGCAAAGTTGGGGCAAGTTAA GCACTGAAGCAGACATACTCTCAGGAAATCCCAAGAGTGCAACGCACGTTCAGGAGG CCATTGGTTCACGGAGTTCTGCAATT

TTGTGAATTGCAGAACTCCGTGAACCAATGGCCTCCTGAACGTGCGTTGCACTCTTGG GATTTCCTGAGAGTATGTCTGCTTCAGTGCTTAACTTGCCCCAACTTTGCAAGCAGGAT GTGTTTCTGCCTTGCGTTCTTATGAGCTATTGCCCTCTGAGCCAATGGCTTGTTAATTGC TTGGTTCTTGCAAAATGCTTTGCGCGCTGTTATTCAGGTTTCTACCTTCGTGGTTTTACT TGAGTGACGCTGCTCATGCTTGCAACCGCTGGGATGCAGGTGCATGCCTCTAGCATGA AGTCAGACAAGTGAACCCGCTGAATTTAAGCATATAAGTAAGCGGAGGACT 
Appendix

Symbiodinium type C3 (Acropora millepora)

AACCAATGGCCTCCTGAACGTGCGTTGCACTCTTGGGATTTCCTGAGAGTATGTCTGCT TCAGTGCTTAACTTGCCCCAACTTTGCAAGCAGGATGTGTTTCTGCCTTGCGTTCTTAT GAGTTATTGCCCTCTGAGCCAATGGCTTGTTAATTGCTTGGTTCTTGCAAAATGCTTTG CGCGCTGTTATTCAAGTTTCTACCTTCGTGGTTTTACTTGAGTGACGCTGCTCATGCTTG CAACCGCTGGGATGCAGGTGCATGCCTCTAGCATGAAGTCAGACAA

AACCAATGGCCTCCTGAACGTGCGTTGCACTCTTGGGATTTCCTGAGAGTATGTCTGCT TCAGTGCTTAACTTGCCCCAACTTTGCAAGCAGGATGTGTTTCTGCCTTGCGTTCTTAT GAGCTATTGCCCTCTGAGCCAATGGCTTGTTAATTGCTTGGTTCTTGCAAAATGCTTTG CGCGCTGTTATTCAAGTTTCTACCTTCGTGGTTTTACTTGAGTGACGCTGCTCATGCTTG CAACCGCTGGGATGCAGGTGCATGCCTCTAGCATGAAGTCAGACAAGTGAACCCGCTG AATTTAAGCATATAA

Symbiodinium type C15 (Montipora digitata)

AACCAATGGCCTCCTGAACGTGCGTTGCACCCTTGGGATTTCCTGAGAGTATGTCTGCT TCAGTGCTTAACTTGCCCCAACTTTGCAAGCAGGATGTGTTTCTGCCTTGCGTTCTTAT GAGCTATTGCCTTCTGCGCCAATGGCTTGTTAATTGCTTGGTTCTTGCAAAATGCTTTG CGCGCTGTTATTCAAGTTTCTACCTTCGCGGTTTTACTTGAGTGACGCTGCTCATGCTT GCAACCGCTGGGATGCAGGTGCATGCCTCTAGCATGAAGTCAGACAA

AACCAATGGCCTCCTGAACGTGCGTTGCACCCTTGGGATTTCCTGAGAGTATGTCTGCT TCAGTGCTTAACTTGCCCCAACTTTGCAAGCAGGATGTGTTTCTGCCTTGCGTTCTTAT GAGCTATTGCCTTCTGCGCCAATGGCTTGTTAATTGCTTGGTTCTTGCAAAATGCTTTG CGCGCTGTTATTCAAGTTTCTACCTTCGCGGTTTTACTTGAGTGACGCTGCTCATGCTT GCAACCGCTGGGATGCAGGTGCATGCCTCTAGCATGAAGTCAGACAA

Symbiodinium type C15 (Porites cylindrica)

AACCAATGGCCTCCTGAACGTGCGTTGCACCCTTGGGATTTCCTGAGAGTATGTCTGCT TCAGTGCTTAACTTGCCCCAACTTTGCAAGCAGGATGTGTTTCTGCCTTGCGTTCTTAT GAGCTATTGCCTTCTGCGCCAATGGCTTGTTAATTGCTTGGTTCTTGCAAAATGCTTTG CGCGCTGTTATTCAAGTTTCTACCTTCGCGGTTTTACTTGAGTGACGCTGCTCATGCTT GCAACCGCTGGGATGCAGGTGCATGCCTCTAGCATGAAGTCAGACAA 
Appendix

Appendix B - Additional chlorphyll fluorescence data (Chapter 2)

B.1 - Additional graphs for thermal stress experiment

Symbiodinium type B2
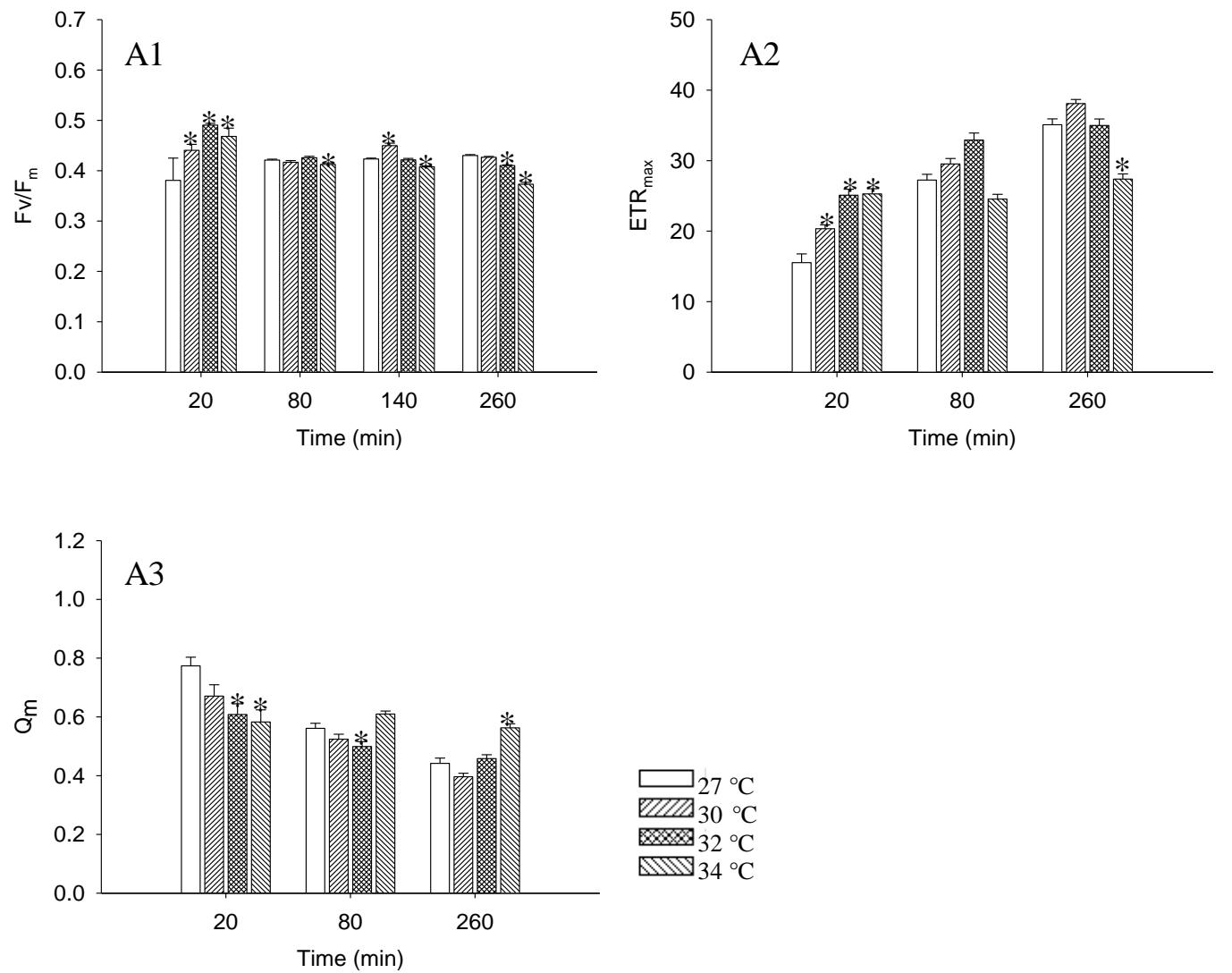
Appendix

Symbiodinium type E1-O
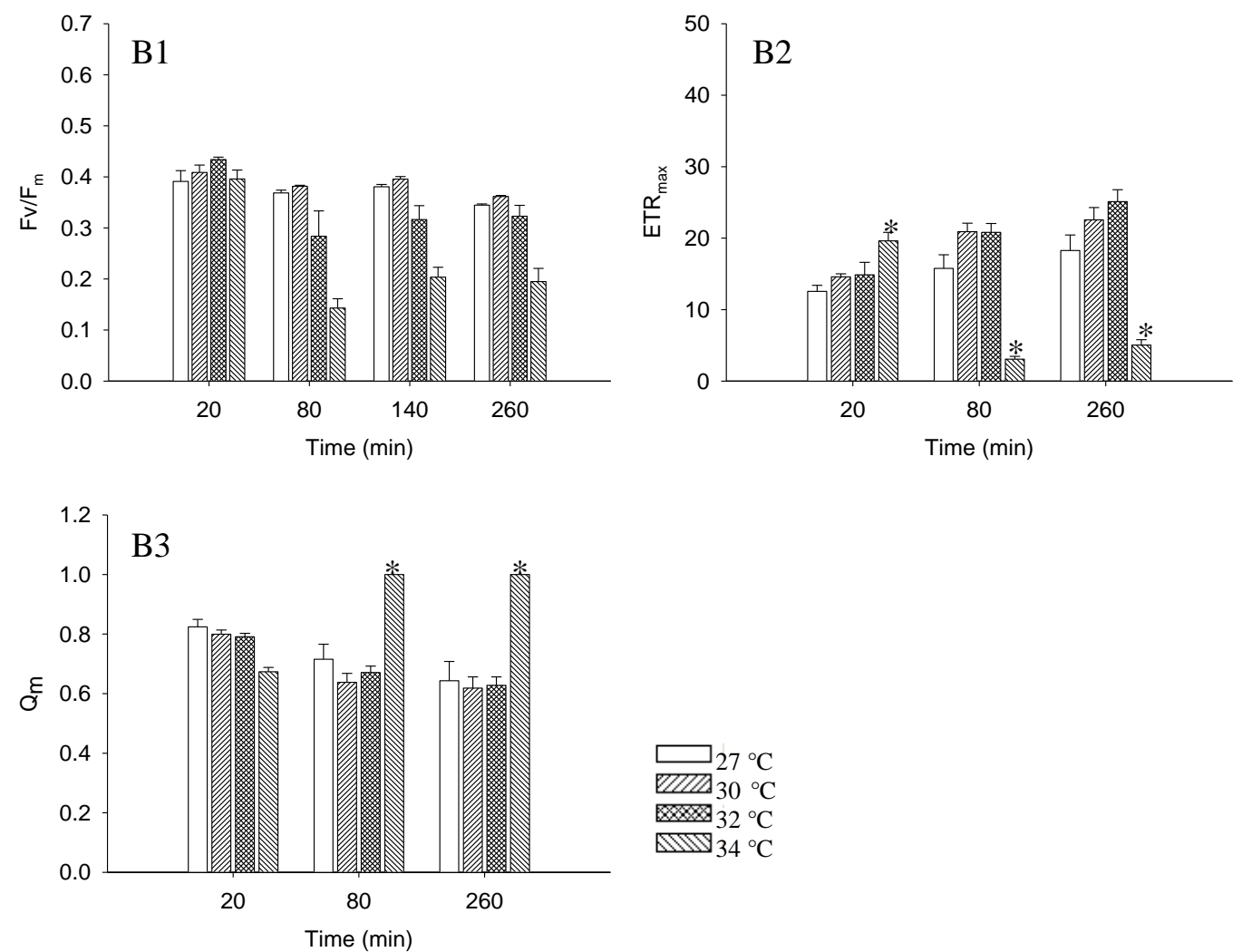

Symbiodinium type F1-P
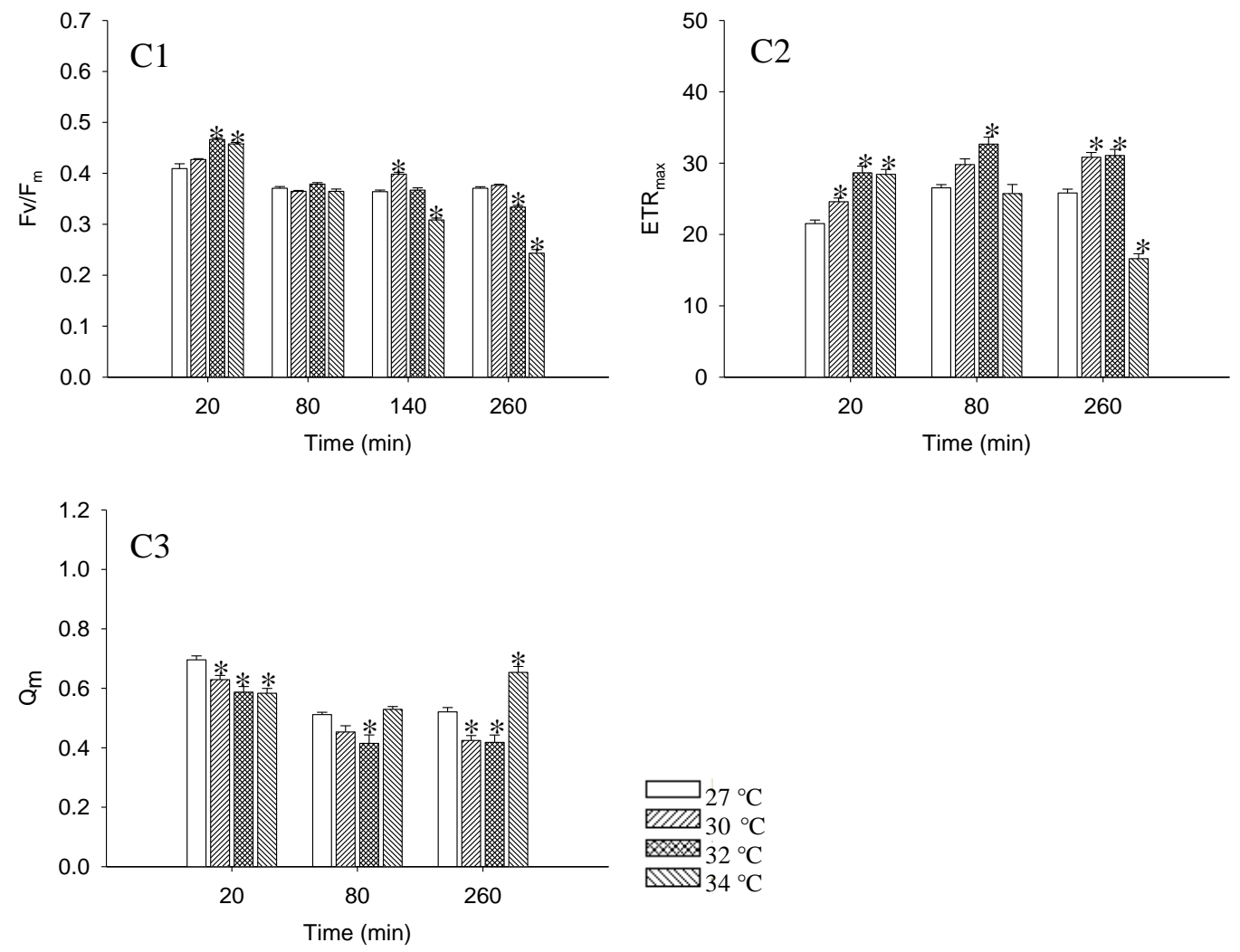
Appendix

Symbiodinium type $S$. californium
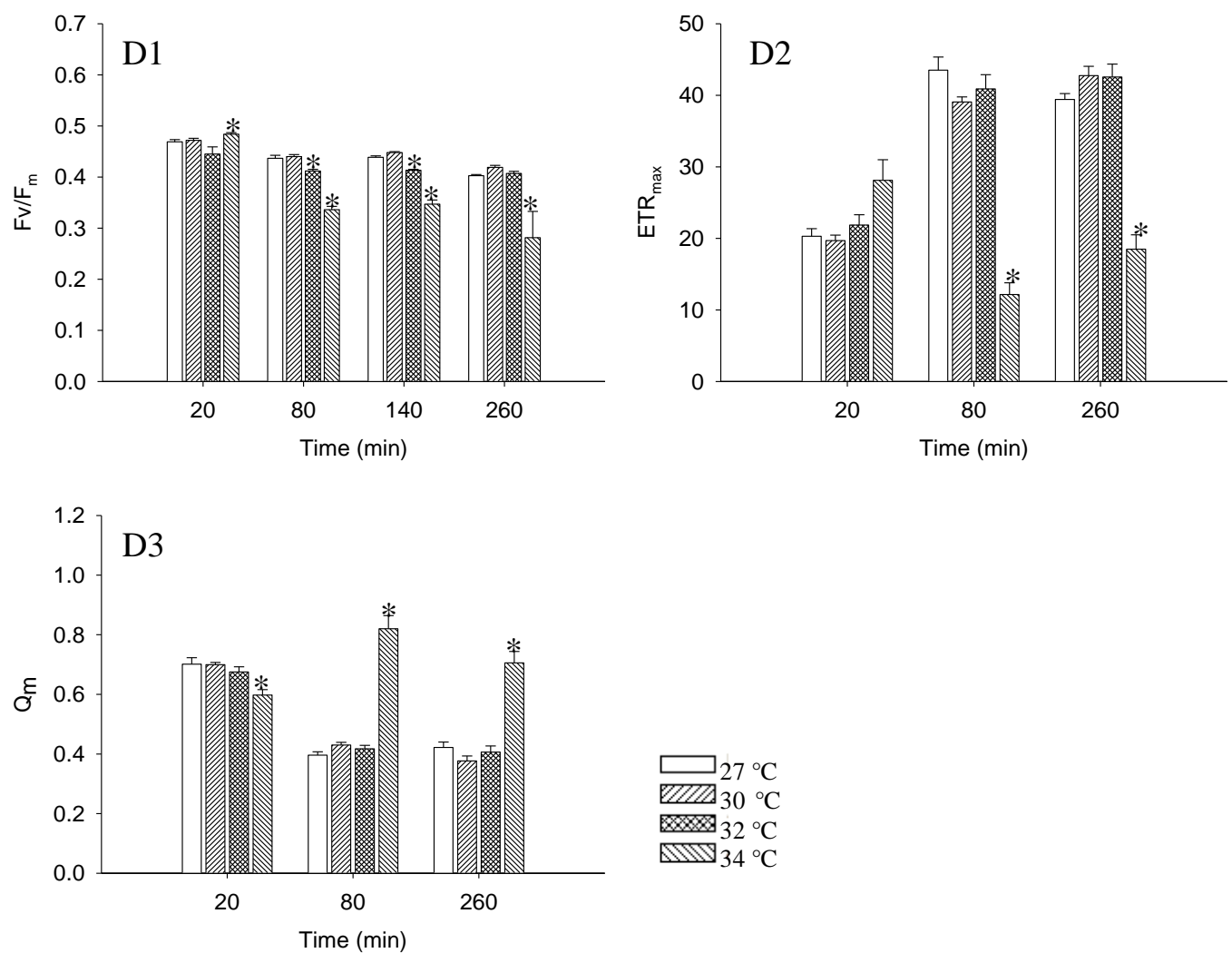

Symbiodinium type A1-FIS
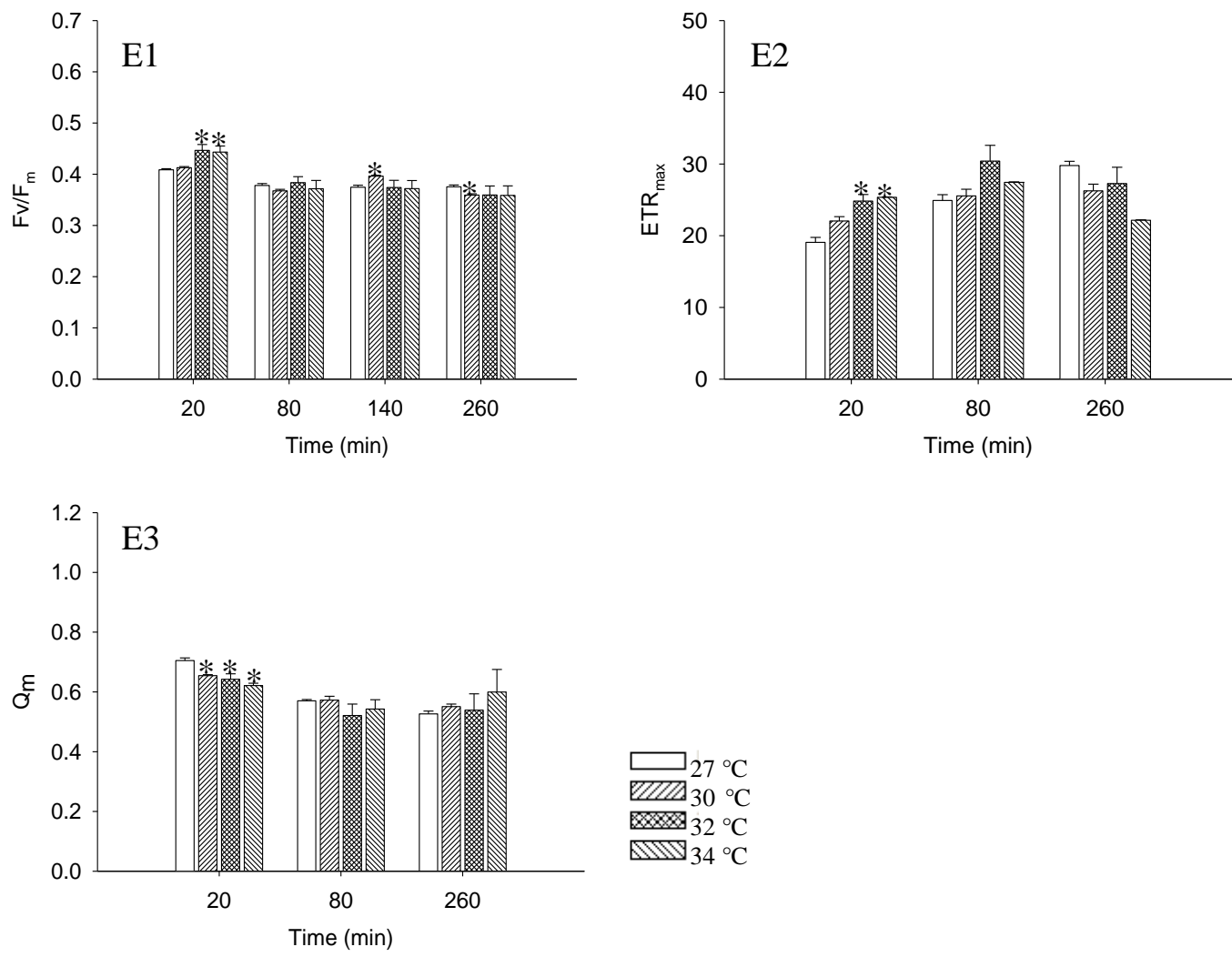
Appendix

Symbiodinium type B1-FIS
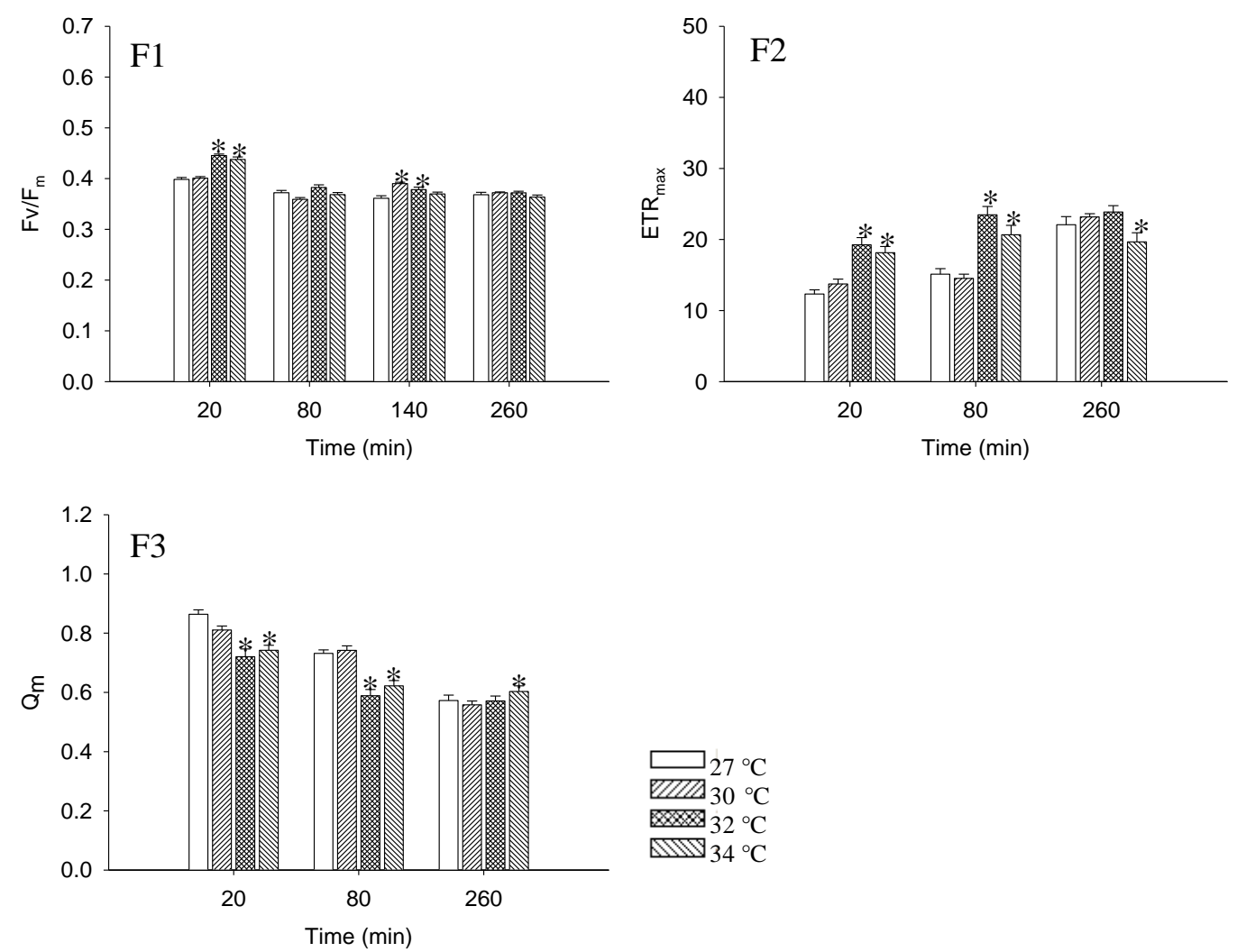

Figure B.1: Effect of increasing temperature $\left(27,30,32,34{ }^{\circ} \mathrm{C}\right)$ on photosynthetic capacity of Symbiodinium types; (A1-F1) maximum quantum yield $\left(\mathrm{F}_{\mathrm{v}} / \mathrm{F}_{\mathrm{m}}\right)$; (A2-F2) maximum rate of relative electron transport $\left(\mathrm{ETR}_{\max }\right)$; (A3-F3) maximum excitation pressure over photosystem II $\left(\mathrm{Q}_{\mathrm{m}}\right)$. Averages $( \pm$ SE) shown $(n=5)$. Asterisks indicate significant differences $(p<0.05)$ between control $(0 \mathrm{mM})$ and treatments. 
Appendix

\section{B.2 - Additional graphs for oxidative stress experiment}

Symbiodinium type A1
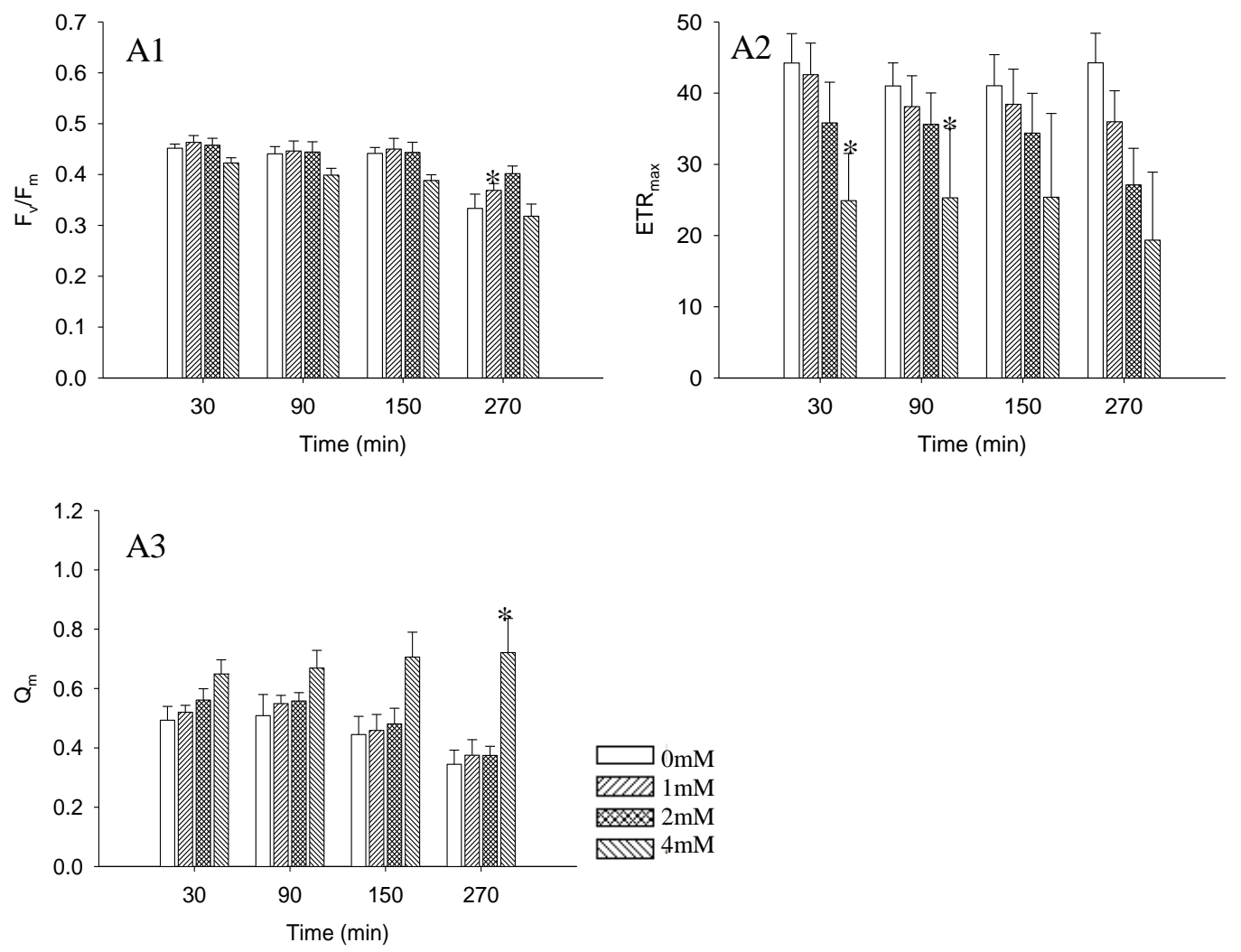
Appendix

Symbiodinium type B2
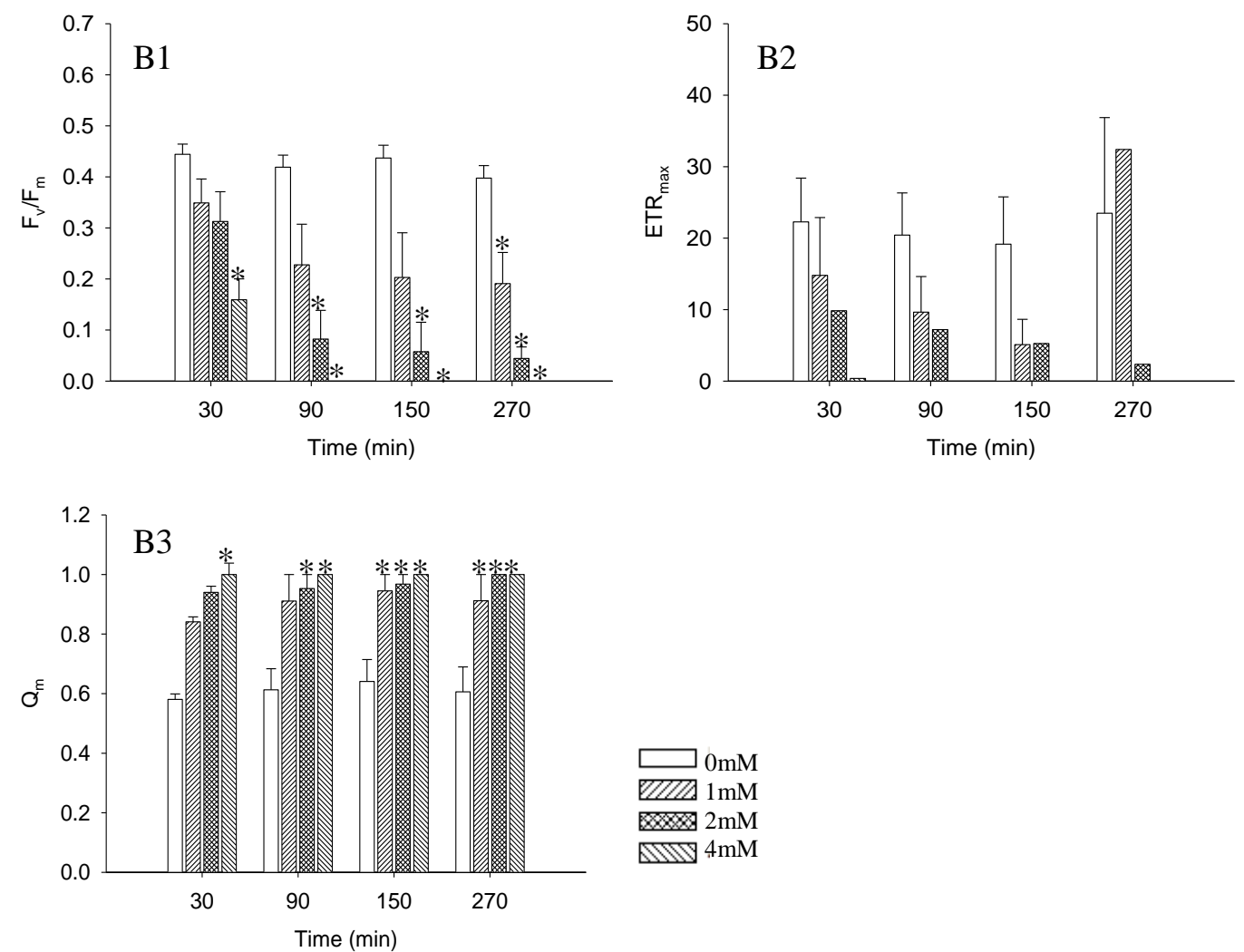

Symbiodinium type E1-O
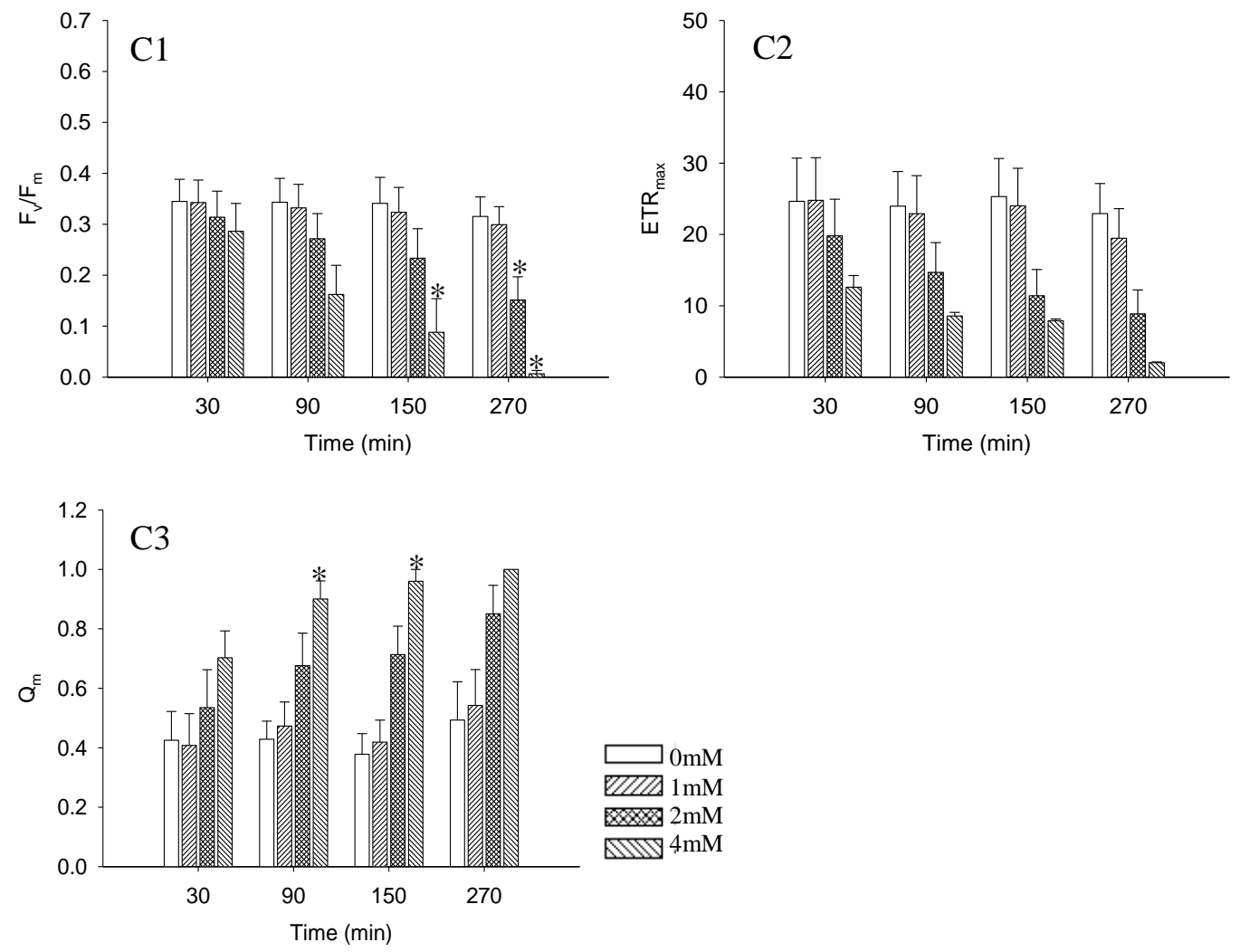
Appendix

Symbiodinium type F1-P


Symbiodinium type $S$. californium
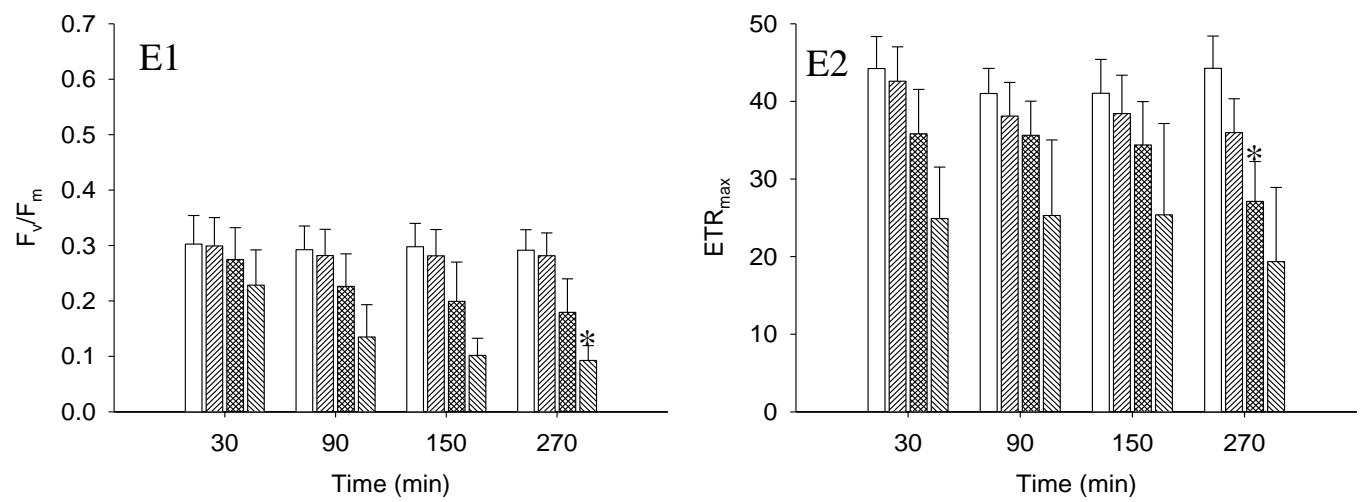


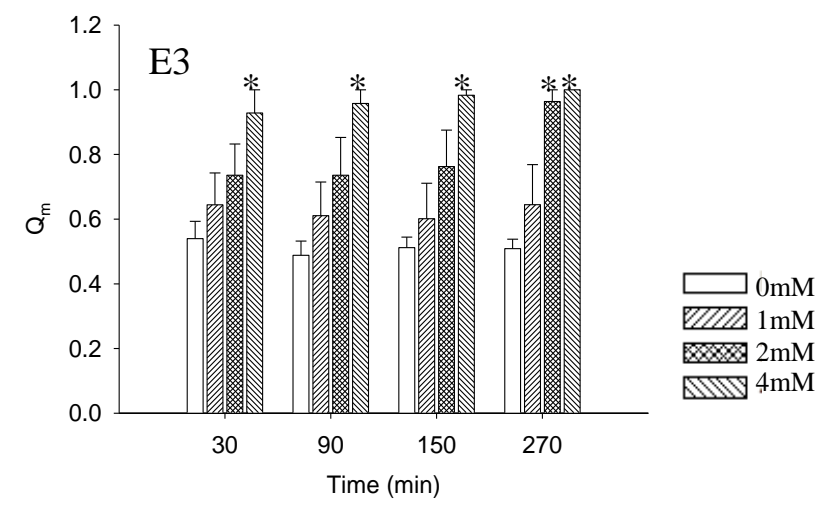

Symbiodinium type B1-FIS
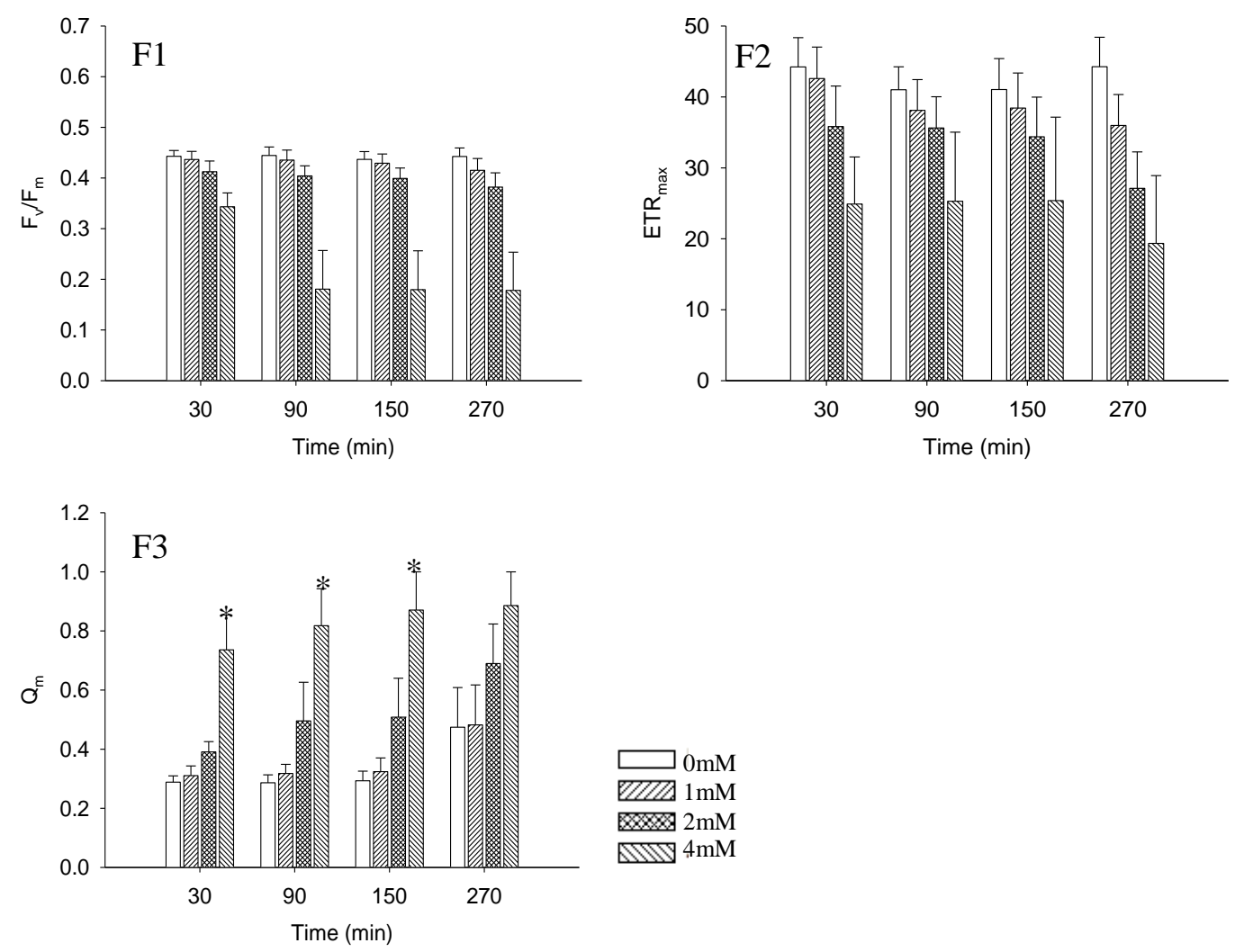

Figure B.2: Effect of increasing hydrogen peroxide $\left(\mathrm{H}_{2} \mathrm{O}_{2} ; 0,1,2,4 \mathrm{mM}\right)$ on photosynthetic capacity of Symbiodinium type over time; (A1-F1) maximum quantum yield $\left(\mathrm{F}_{\mathrm{v}} / \mathrm{F}_{\mathrm{m}}\right)$; (A2-F2) maximum rate of relative electron transport $\left(\mathrm{ETR}_{\max }\right)$; (A3-F3) maximum excitation pressure over photosystem II $\left(\mathrm{Q}_{\mathrm{m}}\right)$; (A4-E4) oxygen fluxes $\left(\mathrm{mg} \mathrm{C} \mathrm{mm}^{-3} \mathrm{~h}^{-1}\right)$ Averages \pm SE shown $(\mathrm{n}=5)$. Asterisks indicate significant differences $(\mathrm{p}<0.05)$ between control $(0 \mathrm{mM})$ and treatments. 
Appendix

B.3 - Additional graphs for oxidative stress experiments with other culture strains

Culture strain Zs
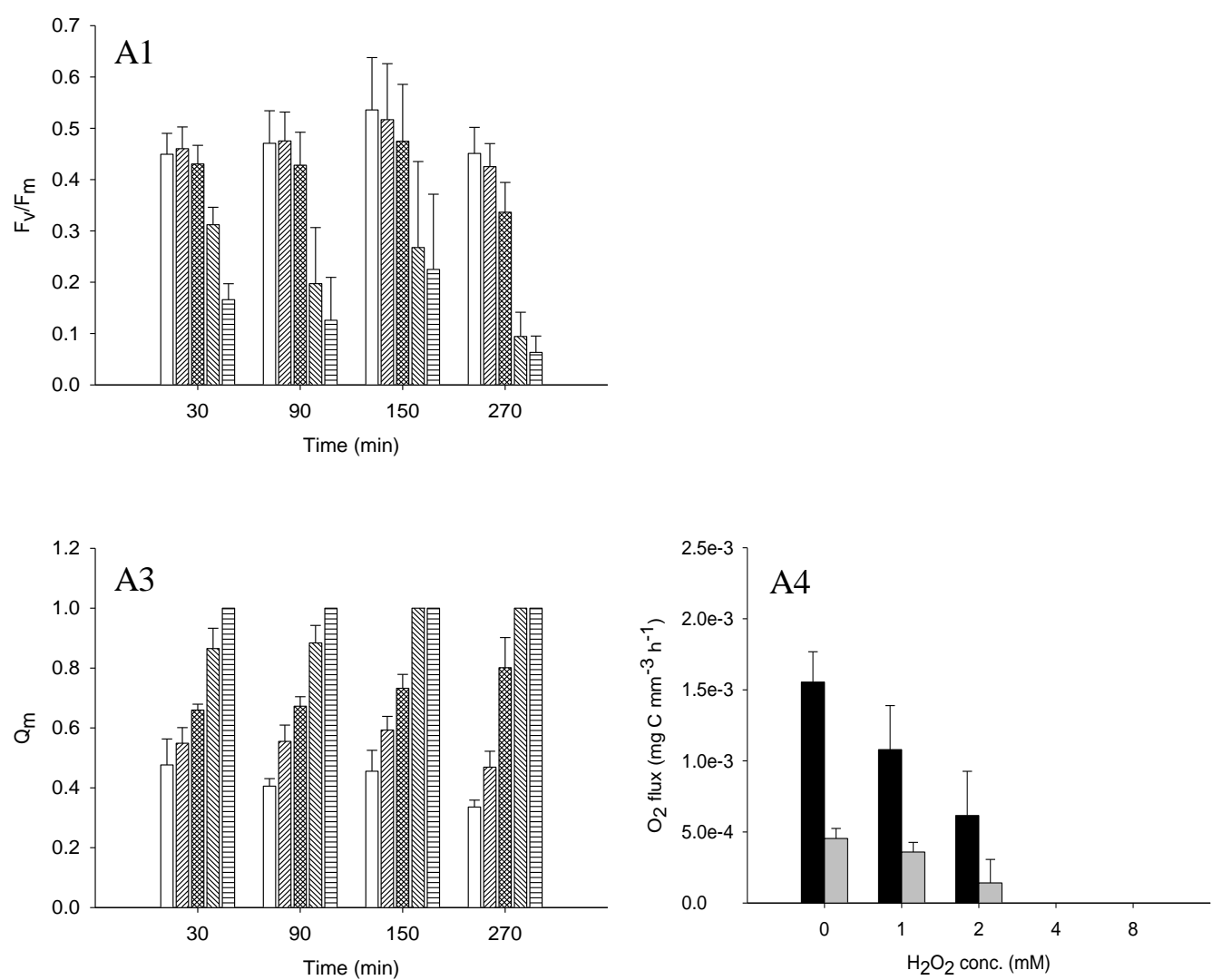

(A1-3) $\square: 0 \mathrm{mM}$; एव: $1 \mathrm{mM}$; : $2 \mathrm{mM}$; $: 4 \mathrm{mM}$; $\square: 8 \mathrm{mM} \mathrm{H}_{2} \mathrm{O}_{2}$ (A4) black: Photosynthetic production; grey: Respiration 
Appendix

Culture strain Ap1
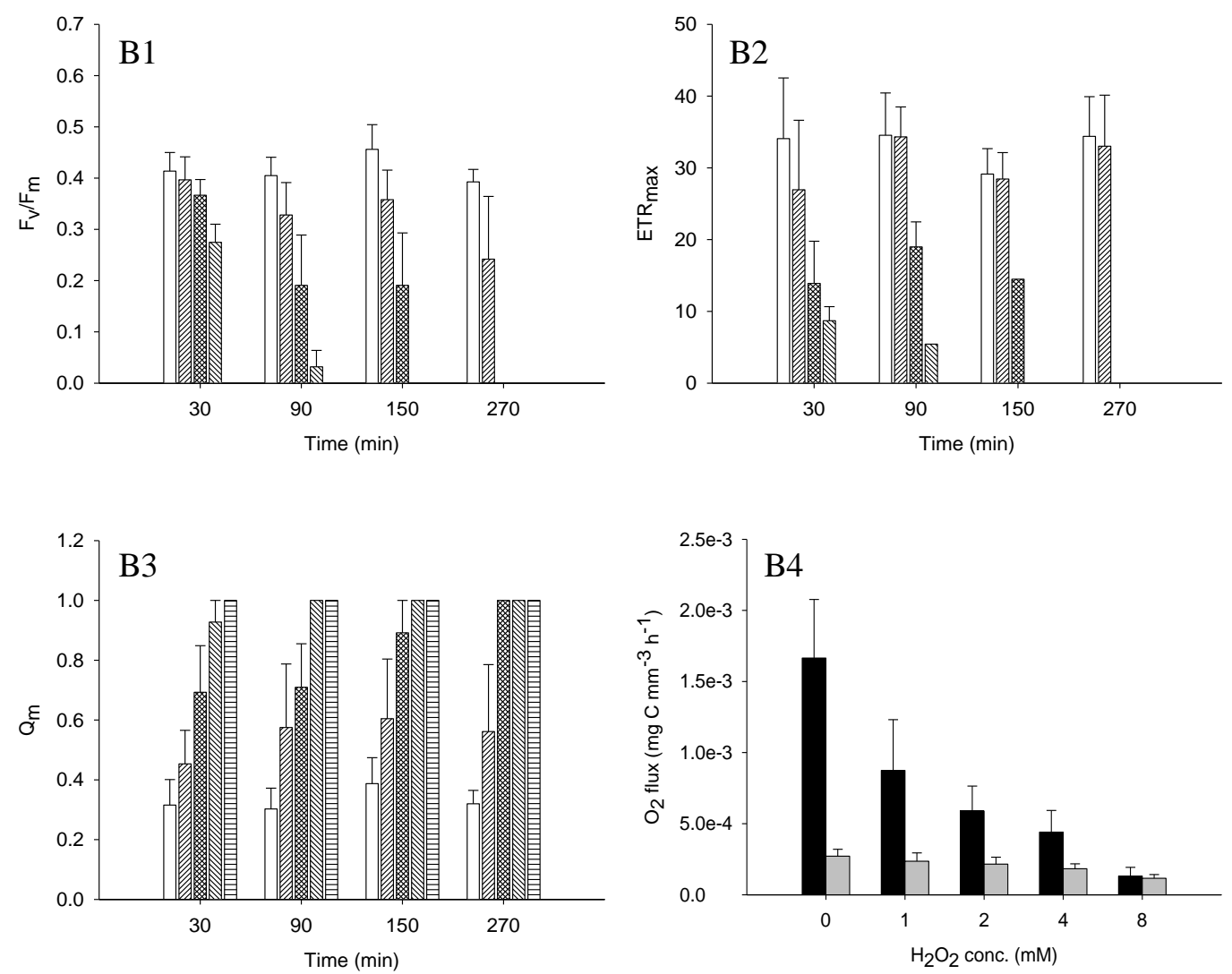

(A1-3) $\square: 0 \mathrm{mM}$; एाय : $1 \mathrm{mM}$; $: 2 \mathrm{mM}$; $: 4 \mathrm{mM}$; (A4) black: Photosynthetic production; grey: Respiration 
Appendix

Symbiodinium type S. bermudense


(A1-3) $\square: 0 \mathrm{mM}$; एाय : $1 \mathrm{mM}$; : $2 \mathrm{mM}$; $: 4 \mathrm{mM}$; $\square: 8 \mathrm{mM} \mathrm{H}_{2} \mathrm{O}_{2}$ (A4) black: Photosynthetic production; grey: Respiration 
Appendix

Culture strain Ap2
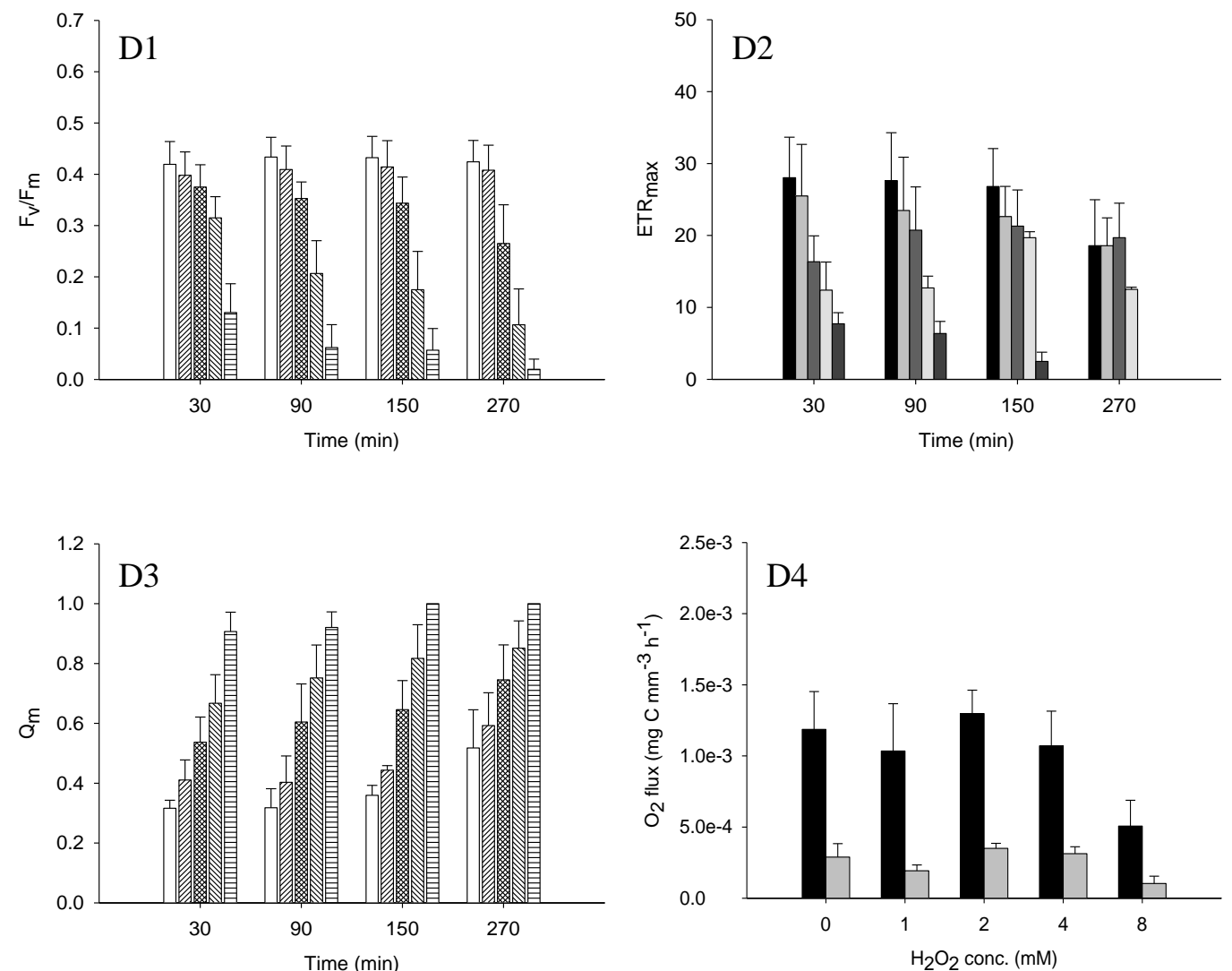

(A1-3) $\square: 0 \mathrm{mM}$; एया : $1 \mathrm{mM}$; $: 2 \mathrm{mM}$; $: 4 \mathrm{mM}$; $\rightleftharpoons: 8 \mathrm{mM} \mathrm{H}_{2} \mathrm{O}_{2}$ (A4) black: Photosynthetic production; grey: Respiration 
Appendix

Culture strain Pd45a
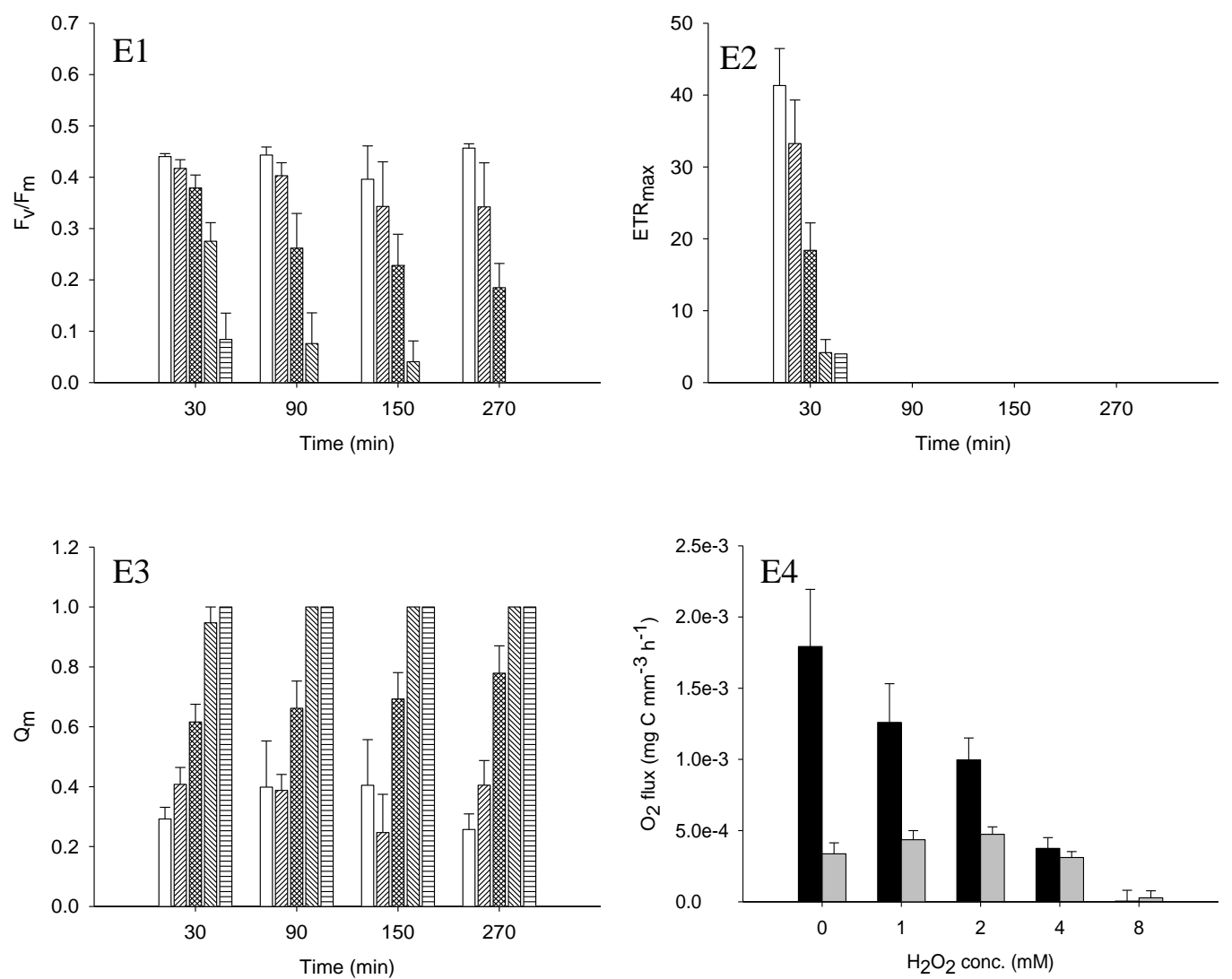

(A1-3) $\square: 0 \mathrm{mM}$; एव: $1 \mathrm{mM}$; : $2 \mathrm{mM}$; $: 4 \mathrm{mM}$;

(A4) black: Photosynthetic production; grey: Respiration

Figure B.3: Effect of increasing hydrogen peroxide $\left(\mathrm{H}_{2} \mathrm{O}_{2} ; 0,1,2,4 \mathrm{mM}\right)$ on photosynthetic capacity of Symbiodinium type over time; (A1-E1) maximum quantum yield $\left(\mathrm{F}_{\mathrm{v}} / \mathrm{F}_{\mathrm{m}}\right)$; (B2-E2) maximum rate of relative electron transport $\left(\mathrm{ETR}_{\max }\right)$; (A3-E3) maximum excitation pressure over photosystem II ( $\left.\mathrm{Q}_{\mathrm{m}}\right)$; (A4-E4) oxygen fluxes $\left(\mathrm{mg} \mathrm{C} \mathrm{mm}^{-3} \mathrm{~h}^{-1}\right)$ with photosynthetic production (black bar) and respirometric consumption (light grey bars). Averages \pm SE shown $(n=5)$. 
Appendix

\section{Appendix C - Additional FRAP and CAA data (Chapter 4)}

\section{C.1 - FRAP assay}

Symbiodinium type A1-FIS
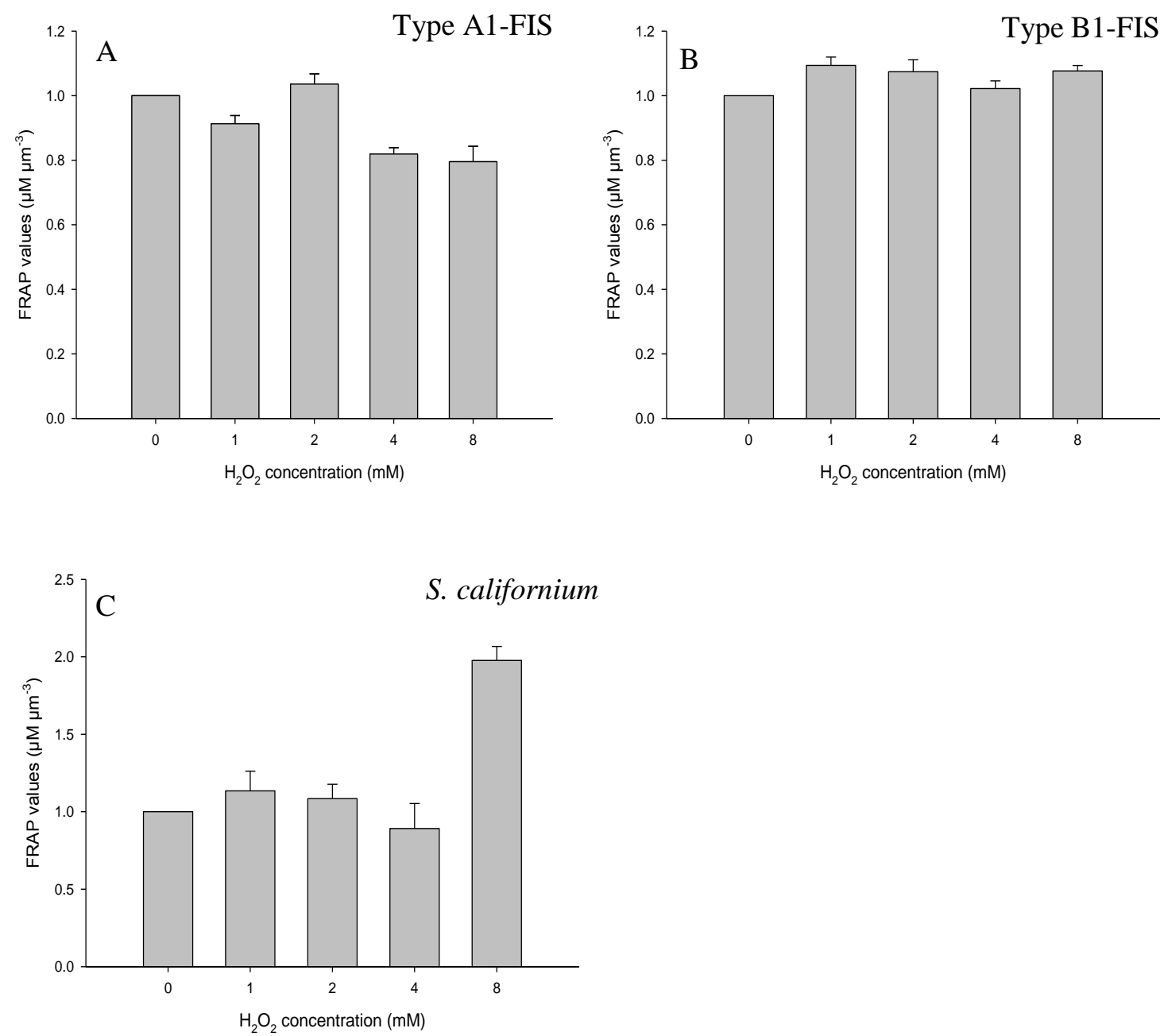

Figure C.1: Ferric reducing antioxidant potential (FRAP) values per cell volume $\left(\mu \mathrm{M} \mathrm{m}^{-3}\right)$ for three Symbiodinium types, (A) A1-FIS; (B) B1-FIS; (C) S. californium. Results are given in relation to the control set as $1(n=5$, means \pm SE). 
Appendix

\section{C.2 - CAA assay}
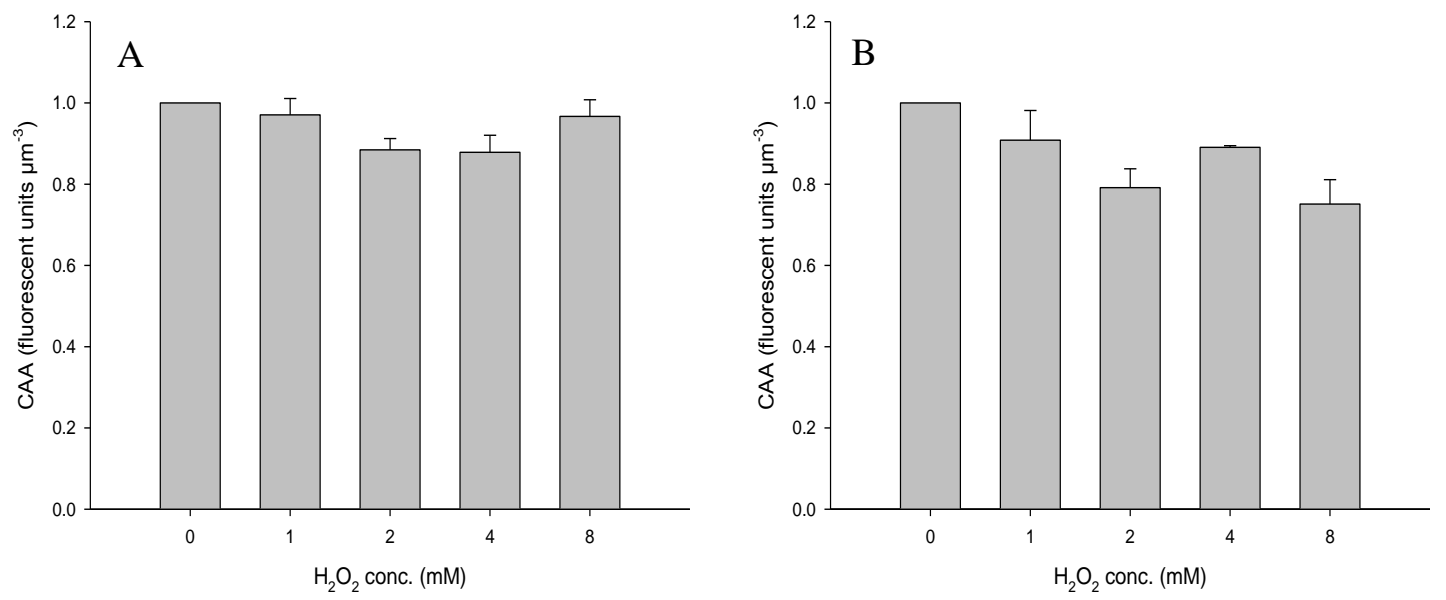

Figure C.2: Cellular antioxidant assay (CAA) fluorescent units per cell volume for two Symbiodinium types, (A) A1-FIS; (B) B1-FIS. Results are given in relation to the control of each type set $a_{b} 1(n=4$; mean $\pm S E)$. 